\title{
Quantitative morphologic and functional MRI of peripheral arterial disease
}

Citation for published version (APA):

Versluis, B. (2014). Quantitative morphologic and functional MRI of peripheral arterial disease. [Doctoral Thesis, Maastricht University]. Datawyse / Universitaire Pers Maastricht.

https://doi.org/10.26481/dis.20140703bv

Document status and date:

Published: 01/01/2014

DOI:

10.26481/dis.20140703bv

Document Version:

Publisher's PDF, also known as Version of record

\section{Please check the document version of this publication:}

- A submitted manuscript is the version of the article upon submission and before peer-review. There can be important differences between the submitted version and the official published version of record.

People interested in the research are advised to contact the author for the final version of the publication, or visit the DOI to the publisher's website.

- The final author version and the galley proof are versions of the publication after peer review.

- The final published version features the final layout of the paper including the volume, issue and page numbers.

Link to publication

\footnotetext{
General rights rights.

- You may freely distribute the URL identifying the publication in the public portal. please follow below link for the End User Agreement:

www.umlib.nl/taverne-license

Take down policy

If you believe that this document breaches copyright please contact us at:

repository@maastrichtuniversity.nl

providing details and we will investigate your claim.
}

Copyright and moral rights for the publications made accessible in the public portal are retained by the authors and/or other copyright owners and it is a condition of accessing publications that users recognise and abide by the legal requirements associated with these

- Users may download and print one copy of any publication from the public portal for the purpose of private study or research.

- You may not further distribute the material or use it for any profit-making activity or commercial gain

If the publication is distributed under the terms of Article $25 \mathrm{fa}$ of the Dutch Copyright Act, indicated by the "Taverne" license above, 


\section{QUANTITATIVE MORPHOLOGIC AND FUNCTIONAL MRI OF PERIPHERAL ARTERIAL DISEASE}

Bastiaan Versluis 
Ccopyright Bastiaan Versluis, Maastricht 2014 ISBN 9789461593382

Printing: Datawyse / Universitaire Pers Maastricht Omslagontwerp: Alice Huisman \& Janneke Scheerhorn 


\section{Quantitative \\ morphologic and \\ functional MRI of \\ peripheral arterial disease}

Proefschrift

Ter verkrijging van de graad van doctor aan de

Universiteit Maastricht

op gezag van de Rector Magnificus Prof. dr. L.L.G. Soete,

volgens het besluit van het College van Decanen,

in het openbaar te verdedigen

op donderdag 3 juli 2014 om 14:00 uur

door

Bastiaan Versluis

Geboren op 24-02-1983 te Eindhoven

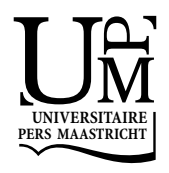




\section{Promotores}

Prof. dr. ir. W.H. Backes

Prof. dr. J.E. Wildberger

\section{Co-promotores}

Dr. T. Leiner, UMC Utrecht

Dr. P.J. Nelemans

\section{Beoordelingscommissie}

Prof. dr. (em) J.M.A. van Engelshoven (voorzitter)

Prof. dr. M.W. de Haan

Prof. dr. H. J. Lamb, Leiden UMC

Dr. R.J.M.W. Rennenberg

Prof. dr. ir. P.F.F. Wijn, TU Eindhoven, Maxima Medisch Centrum 


\section{Contents}

Chapter $1 \quad$ General introduction 7

Chapter $2 \quad$ MRI in peripheral arterial disease: Reproducibility of the assessment of morphological and functional vascular status

Chapter 3 MRI derived arterial peak flow in peripheral arterial disease: Towards a standardized measurement

Chapter 4 Functional MRI in peripheral arterial disease: Arterial peak flow versus ankle-brachial index

Chapter $5 \quad$ Reference values for peripheral arterial peak flow in patients with peripheral arterial disease: Assessment by phase-contrast magnetic resonance flow imaging

Chapter $6 \quad$ Magnetic resonance imaging-based monitoring of collateral artery development in patients with intermittent claudication during supervised exercise therapy

Chapter $7 \quad$ MRI of arterial flow reserve in patients with intermittent claudication: Feasibility and initial experience

Chapter 8 Dynamic contrast-enhanced MRI assessment of hyperemic fractional microvascular blood plasma volume in peripheral arterial disease: Initial findings

Chapter 9

General discussion

Appendix

Summary / samenvatting

Dankwoord

Curriculum vitae

Publications 



\section{CHAPTER 1}

General introduction 

Arteriosclerotic peripheral arterial disease (PAD) is a major health care problem among the elderly in the Western society. The estimated prevalence of PAD in the general population is in the range of $3 \%$ to $10 \%$, which increases up to $15-20 \%$ in persons over 70 years $(1-4)$. In the Netherlands, the estimated prevalence among persons over 55 years is $19.1 \%$, ranging from $8.1 \%$ at the age of $55-59$ years towards $55.8 \%$ in persons over 85 years (5). The prevalence in males and females is roughly the same in the Netherlands (16.9\% versus $20.5 \%$, respectively), although men are more prone to develop symptomatic PAD (6).

PAD is an expression of generalized arteriosclerosis, caused by well-known risk factors like smoking, diabetes mellitus, hypertension, dyslipidemia and hyperhomocysteinemia (4), and clinical symptoms are the result of compromised arterial flow to the lower extremities due to obstructive peripheral arterial lesions (figure 1). Besides obstructive arterial lesions at the macrovascular level, i.e. lesions of the large conduit arteries of the lower extremities, disorders might also be present at the microvascular level, in which small arteries, arterioles and capillaries are affected. Microvascular disease comprises endothelial and vascular smooth muscle cell dysfunction and inflammation, which results in a disturbed production of vaso-active substances, leading to increased vascular tone and damage $(1,7-10)$. Microvascular disease is most pronounced in the majority of the subgroup of PAD patients with diabetes mellitus, whereas macrovascular disease is the major cause of symptoms in non-diabetic PAD patients (1, 7-10).

Symptoms in PAD range from intermittent claudication, commonly defined as pain and / or cramping of the calf musculature in response to exercise, relieved by a short period of rest, to critical limb ischemia, defined as ischemic rest pain, ulceration or gangrene (11). Although intermittent claudication is the most common clinical expression of PAD, the vast majority of PAD patients are asymptomatic (symptomatic versus asymptomatic ratio is in the range of $1: 3$ to $1: 4$ ) and unknown to clinicians $(4,11)$. Nevertheless, some patients with asymptomatic PAD may have greater functional impairment and more adverse pathophysiologi- 
cal findings in calf muscles and lower extremity nerves compared to PAD patients with intermittent claudication (12).

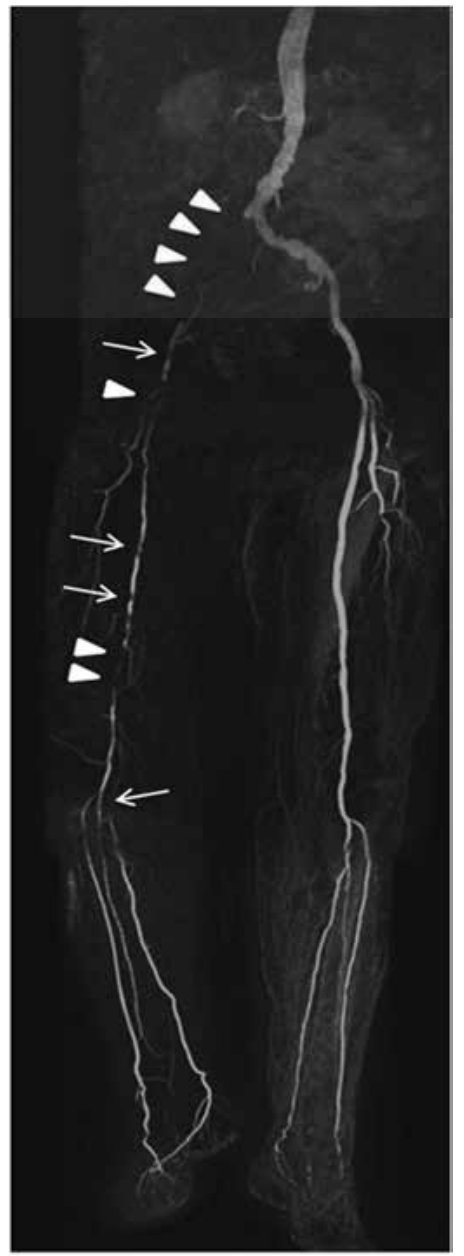

Figure 1. Contrast-enhanced MR angiography of the peripheral arterial tree in a patient with PAD. There are numerous occlusions (arrowheads) and stenosis (arrows) present in the right leg, whereas the arteries of the left leg show signs of arterial disease as well.
Although PAD frequently is a progressive disease, only a fraction of asymptomatic subjects harboring the disease will ever become symptomatic. In approximately three quarters of the patients with intermittent claudication clinical symptoms are stable over time, which may be due to the development of new arterial structures, bypassing the original obstructed artery, the so-called collateral arteries (13). Collateral artery formation following peripheral ischemia is a complex process, the details of which are increasingly being elucidated (14). However, in depth research studies in human PAD patients on the potential vascular mechanisms underlying rehabilitation are limited and have provided conflicting results up to now (13). The formation of collateral arteries could thus far not be visualized or quantified and their specific contribution has not been conclusively determined. The exact mechanisms underlying collateral formation are still unknown, as well as the reasons why the extent of collateral formation can differ from one patient to another. In $25 \%$ of the patients with intermittent claudication symptoms will significantly deteriorate, most frequently during the first year after the diagnosis (4). However, the prognosis is generally good and less than $2 \%$ of patients with PAD will end-up with major limb amputations, which is a major fear of 
many patients $(4,15)$. Unfortunately, to date it is difficult to predict which PAD patients will significantly deteriorate, although it is known that continuing smoking and diabetes mellitus have a negative impact on the prognosis of PAD patients.

\section{Diagnosis and treatment of peripheral arterial disease}

The diagnosis of PAD is made on the basis of the typical clinical history, palpation of pedal pulsations and by measurement of the anklebrachial index $(A B I)(4,16)$. Once established, the severity of PAD is graded by using either the Fontaine or Rutherford classifications (table 1) $(12,17,18)$. Measurement of $A B I$ is a cheap and ubiquitously available screening tool in the high-risk, asymptomatic population to confirm the presence of PAD, and is clinically relevant because of the strong association with underlying but clinically silent coronary artery disease $(4,11,19)$.

\begin{tabular}{|c|c|c|c|c|}
\hline \multicolumn{2}{|c|}{ Fontaine classification } & \multicolumn{3}{|c|}{ Rutherford classification } \\
\hline Stage & Symptoms & Grade & Category & Symptoms \\
\hline 1 & Asymptomatic & 0 & 0 & Asymptomatic \\
\hline Ila & Mild claudication* & 1 & 1 & Mild claudication* \\
\hline \multirow{2}{*}{$\mathrm{IIb}$} & Moderate - severe* & 1 & 2 & Moderate claudication* \\
\hline & claudication & 1 & 3 & Severe claudication* \\
\hline III & Ischemic rest pain & II & 4 & Ischemic rest pain \\
\hline \multirow[t]{2}{*}{ IV } & Ulceration or gangrene & III & 5 & Tissue ulceration \\
\hline & & IV & 6 & Tissue loss/gangrene \\
\hline
\end{tabular}

Table 1. Overview of the Fontaine and Rutherford classification system.

* Mild, moderate and severe claudication are not further specified for the Rutherford classification, whereas in the Fontaine classification mild and moderate to severe claudication differ in a pain free walking distance of more (mild claudication) or less (moderate to severe claudication) than 200 meters.

The treatment of PAD is primarily aimed at relieving symptoms. Given the relative benign course of PAD, today's consensus on the best initial approach is optimized medical treatment in asymptomatic sub- 


\section{CHAPTER 1}

jects (detected by screening) and, in addition, supervised exercise training in patients with intermittent claudication. For asymptomatic patients treatment comprises risk factor modification to prevent further progression of $\operatorname{PAD}(4,11)$. In patients with intermittent claudication, the aim of treatment is improvement of exercise performance and daily functional abilities, which can be accomplished by structured exercise therapy $(4,13,20,21)$. Only when a patient's complaints do not improve under conservative treatment, or even deteriorate, imaging and invasive therapy (i.e. endovascular or surgical revascularization) are considered $(4,11)$.

Besides the aforementioned therapies, a whole range of novel therapeutic strategies are being developed - primarily for patients with severe PAD unable or unlikely to benefit from conventional approaches which include administration of growth factors and stem cells (22-25). Similar to exercise therapy, those new therapies aim to ameliorate symptoms by stimulation of vascular adaptations, both at the macroand microvascular levels (13, 26-29). At the macrovascular level, these adaptations include the formation or further maturation of collateral arteries and to increase of capacity of existing arteries. Unfortunately, it is unknown to what extent exercise therapy as well as the above mentioned novel therapeutic options contribute to the process of collateral formation, nor is it known if and to what extent these collaterals add to functional improvement in patients. These uncertainties are partly the result of a lack of non-invasive diagnostic methods to visualize and quantify this complex biological process.

\section{Imaging of PAD}

As outlined above, the diagnosis of PAD can be established with virtual certainty based on patient history, clinical symptoms and noninvasive tests like $A B I$. Consequently, imaging of the peripheral vascular tree is only preserved for patients being considered for invasive therapy with the aim to localize arterial lesions and to plan the correct approach for the intervention (figure 1$)(4,11,30)$. Today, the most reliable noninvasive imaging techniques for this purpose are computed tomographic 
angiography (CTA) and magnetic resonance angiography (MRA) (31). Intra-arterial digital subtraction angiography (DSA), the traditional reference standard, is used less and less for planning purposes due to its invasiveness. Both CTA and MRA techniques yield highly accurate morphologic data on the macrovascular status in PAD patients and facilitate assessment of the suitability of lesions for intervention, without the need for invasive DSA (32-34). Major advantages of CTA are high spatial and contrast resolution and fast acquisition times, whereas the most important disadvantages of CTA are the dependency on potential harmful radiation and contrast agents. It is known that many PAD patients suffer from renal artery stenosis as well as renal dysfunction which, with regards to contrast agents, makes CTA a less attractive alternative compared to MRA (35).

MRA, on the other hand, is a relatively new imaging modality for PAD, which has important advantages over CTA. In contrast to CTA, there is no use of iodinated contrast agents, nor is ionizing radiation used. Over the past decade contrast-enhanced MRA (CE-MRA) has established itself as a highly accurate non-invasive diagnostic imaging method for morphological evaluation of the large conduit arteries in PAD patients. Sensitivity and specificity of CE-MRA are comparable with those of invasive intra-arterial DSA $(33,36,37)$. Recently introduced blood pool contrast agents, which reversely bind with blood plasma albumin molecules, thereby limiting extravasation of contrast agent into the interstitial space, increase the temporal imaging window for CEMRA. This allows ultra-high spatial resolution CE-MRA acquisitions and whole-body CE-MRA examinations to be performed with even better diagnostic accuracy (38-42).

Although DSA, CTA and MRA all provide very accurate morphologic information concerning the vascular status, the first two modalities are inherently limited because they only provide morphologic images and little or no information on the hemodynamic consequences of obstructive vascular lesions in PAD patients. Figure 2 for example shows a CE-MRA reconstruction of the superficial femoral artery in a patient with an occluded artery and with extensive collateral artery formation. 


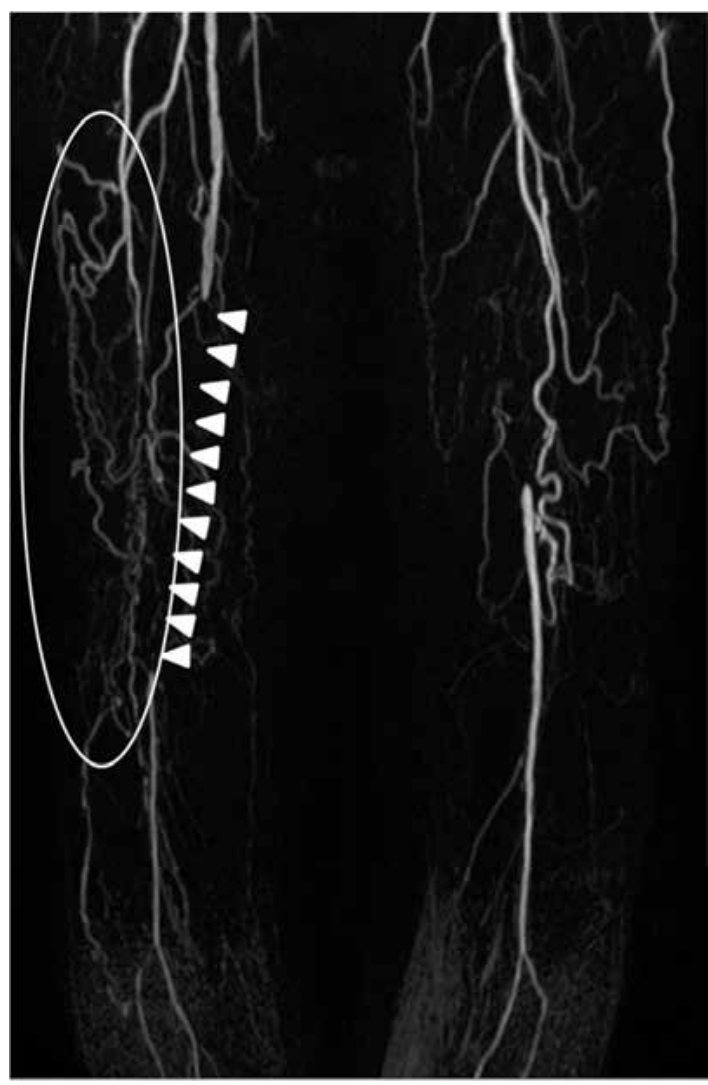

Figure 2. Contrast-enhanced MR angiography of the upper leg of a patient with severe PAD. The superficial femoral artery at both sides is occluded (arrowheads mark occlusion at the right side), whereas especially in the right leg extensive collateral arteries are visible (encircled).
Based on this morphologic image, it is not possible to determine the contribution of these collateral arteries to the blood flow towards the distal lower limb. To supplement morphologic information, it would be highly desirable to use noninvasive imaging tools to determine the hemodynamic significance of arterial stenoses and occlusions as well as the impact of vascular adaptations resulting from different treatment strategies. Combined morphologic and functional methods to visualize and quantify vascular status in PAD patients are useful for several reasons: (i) to confirm the diagnosis and grade the hemodynamic severity of PAD, (ii) for patient follow-up and therapy monitoring, (iii) for studying the mechanisms of underlying vascular adaptations, such as collateral artery formation, and (iv) for studying the effect of present and future therapeutic options. The most attractive imaging modality that has the potential to satisfy all these requirements is MRI. There are numerous MRI techniques besides MRA that are suitable to obtain information on vascular function (43-46). MRI-based functional measurements have the advantage that they can be applied repeatedly 
without detrimental side effects and are relatively easy to add to clinical routine CE-MRA examinations.

\section{Functional MRI in PAD}

One of the most widely used functional MRI measurements at the macrovascular level relevant to PAD is quantitative 2D cine MR phase contrast imaging ( $\mathrm{PCl})$ (44). The temporal variations of both the MR signal phase, induced by the pulsatile arterial blood flow of arteries, are used to obtain quantitative information about blood velocity and flow. The accumulated phase shift is the result of magnetic spins moving along the axis of a flow-encoding magnetic gradient $(47,48)$. PCI detects these phase shifts, which are proportional to blood velocity (44, 49), and can provide a waveform of blood velocity over the cardiac cycle. The blood flow through a blood vessel can then be calculated by summing all velocity values, parallel to the cylinder axis, over all pixel positions of the cross-sectional vessel area depicted in the image.

At the microvascular level, MRI can be used to measure end-organ perfusion utilizing dynamic contrast-enhanced (DCE) MRI, as well as tissue oxygenation using dynamic blood oxygen level-dependent (BOLD) MRI $(43,45)$. DCE MRI is able to determine regional perfusion in skeletal muscle tissue $(50,51)$. By dynamically recording enhancement of muscle tissue during injection of contrast medium and measuring the signal in the arteries supplying those muscles, quantitative information on the microcirculation can be obtained. When a small molecular contrast agent is used, the wash-in rate of the tissue is relatively fast and primarily determined by microvascular flow (and not by vessel wall permeability issues). Using a two-compartment pharmacokinetic model that relates arterial concentration to the tissue concentration, the dynamic transfer of the contrast agent from the blood space to the tissue can be quantified in terms of the unidirectional influx constant, which is a surrogate quantity for microvascular flow.

In contrast to DCE MRI, BOLD MRI is a non-contrast enhanced functional MRI technique to measure perfusion. The BOLD phenomenon is based on local susceptibility changes due to changes in blood oxy- 
genation and BOLD MRI is sensitive to the concentration of paramagnetic deoxyhemoglobin. When the oxygenation state of a certain tissue is altered, the ratio between oxyhemoglobin and deoxyhemoglobin concentrations will change accordingly, which results in a local change in magnetic susceptibility. The presence of deoxyhemoglobin in blood results in a mismatch between the magnetic susceptibility of tissue and blood and thereby shortens the $\mathrm{T}_{2}{ }^{*}$ relaxation time of blood and its immediate surroundings $(45,49)$. Increase of oxygenation increases the measured signal intensity due to the higher oxyhemoglobin and/or lower deoxyhemoglobin concentration. This can be observed under hyperemic (increased blood flow) circumstances, such as reactive hyperemia. Lower signal intensity on the other hand is expected under more ischemic conditions, in which the concentration of deoxyhemoglobin will rise $(45,49,52-57)$.

Functional MRI measurements as described above could in theory provide useful additional quantitative information on the muscle perfusion status in the evaluation of PAD. However, to date the additional value or clinical reproducibility of functional MRI measurements is unknown for PAD.

\section{Objectives}

Main objective of the current thesis was to determine and optimize the suitability of morphologic and functional vascular MRI for diagnosis and longitudinal follow up of PAD patients. To achieve this objective we first determined the reproducibility of CE-MRA as well as the abovementioned functional MRI techniques in PAD patients, non-PAD subjects and healthy controls. We also assessed the discriminative capability of these techniques to differentiate between PAD patients and non-PAD subjects. Based upon these results, improvements of functional vascular MRI techniques and an optimal clinical implementation are proposed and tested in practice. Finally, morphologic and functional vascular MRI was used to evaluate vascular changes over time in PAD patients undergoing supervised exercise therapy. 


\section{Outline of the thesis}

- In chapter 2 the reproducibility of morphologic and functional vascular MRI is evaluated in PAD patients as well as in healthy control subjects. In addition, differences between PAD patients and healthy control subjects are investigated.

- In chapter 3 measures at different levels of the peripheral arterial vasculature in a large population of PAD and non-PAD patients are evaluated to determine the best location to measure the arterial peak flow (APF) in order to facilitate clinical standardization.

- In chapter 4 the APF, success rates and discriminative capability of APF are compared with $A B I$ measurements in a large population of PAD patients.

- In chapter 5 reference values for the APF in patients with PAD and non-PAD subjects are established and the influence of disease severity (intermittent claudication versus critical ischemia), presence of diabetes mellitus and age on APF is evaluated.

- In chapter 6 the results of a longitudinal follow-up study using CEMRA and quantitative $\mathrm{PCl}$ to study the effect of supervised exercise therapy on vascular changes in PAD patients are presented.

- In chapter 7 a technique is presented to measure arterial flow reserve by quantitative $\mathrm{PCl}$ to improve the sensitivity of the APF to differentiate between PAD patients and healthy controls.

- In chapter 8 we demonstrate the feasibility of DCE MRI of the calf musculature of PAD patients and healthy control subjects using a blood pool contrast agent.

- Chapter 9 comprises general conclusions of this thesis.

- Chapter 10 is the summary of the thesis. 


\section{References}

1. Criqui MH, Fronek A, Barrett-Connor E, et al. The prevalence of peripheral arterial disease in a defined population. Circulation. 1985;71(3):510-5.

2. Hiatt WR, Hoag S, Hamman RF. Effect of diagnostic criteria on the prevalence of peripheral arterial disease. The San Luis Valley Diabetes Study. Circulation. 1995;91(5):1472-9.

3. Selvin E, Erlinger TP. Prevalence of and risk factors for peripheral arterial disease in the United States: results from the National Health and Nutrition Examination Survey, 1999-2000. Circulation. 2004;1 10(6):738-43.

4. Norgren L, Hiatt WR, Dormandy JA, et al. Inter-Society Consensus for the Management of Peripheral Arterial Disease (TASC II). J Vasc Surg. 2007;45 Suppl S:S5-67.

5. Stoffers HE, Rinkens PE, Kester AD, et al. The prevalence of asymptomatic and unrecognized peripheral arterial occlusive disease. Int J Epidemiol. 1996;25(2):282-90.

6. Meijer WT, Hoes AW, Rutgers D, et al. Peripheral arterial disease in the elderly: The Rotterdam Study. Arterioscler Thromb Vasc Biol. 1998;18(2):185-92.

7. Huysman E, Mathieu C. Diabetes and peripheral vascular disease. Acta Chir Belg. 2009;109(5):587-94.

8. Chao CY, Cheing GL. Microvascular dysfunction in diabetic foot disease and ulceration. Diabetes Metab Res Rev. 2009;25(7):604-14.

9. Wong WT, Wong SL, Tian XY, et al. Endothelial dysfunction: the common consequence in diabetes and hypertension. J Cardiovasc Pharmacol. 2010;55(4):300-7.

10. Grover-Paez F, Zavalza-Gomez AB. Endothelial dysfunction and cardiovascular risk factors. Diabetes Res Clin Pract. 2009;84(1):1-10.

11. Hirsch AT, Haskal ZJ, Hertzer NR, et al. ACC/AHA 2005 guidelines for the management of patients with peripheral arterial disease (lower extremity, renal, mesenteric, and abdominal aortic): executive summary a collaborative report from the American Association for Vascular Surgery/Society for Vascular Surgery, Society for Cardiovascular Angiography and Interventions, Society for Vascular Medicine and Biology, Society of Interventional Radiology, and the ACC/AHA Task Force on Practice Guidelines (Writing Committee to Develop Guidelines for the Management of Patients With Peripheral Arterial Disease) endorsed by the American Association of Cardiovascular and Pulmonary Rehabilitation; National Heart, Lung, and Blood Institute; Society for Vascular Nursing; TransAtlantic Inter-Society Consensus; and Vascular Disease Foundation. J Am Coll Cardiol. 2006;47(6):1239-312. 
12. McDermott MM, Greenland P, Liu K, et al. Leg symptoms in peripheral arterial disease: associated clinical characteristics and functional impairment. JAMA. 2001;286(13):1599-606.

13. Stewart KJ, Hiatt WR, Regensteiner JG, et al. Exercise training for claudication. N Engl J Med. 2002;347(24):1941-51.

14. Wahlberg E. Angiogenesis and arteriogenesis in limb ischemia. J Vasc Surg. 2003;38(1):198-203.

15. Widmer LK, Biland L. Incidence and course of occlusive peripheral artery disease in geriatric patients. Possibilities and limits of prevention. Int Angiol. 1985;4(3):289-94.

16. Aboyans V, Ho E, Denenberg JO, et al. The association between elevated ankle systolic pressures and peripheral occlusive arterial disease in diabetic and nondiabetic subjects. J Vasc Surg. 2008;48(5):1197-203.

17. Rutherford RB, Baker JD, Ernst C, et al. Recommended standards for reports dealing with lower extremity ischemia: revised version. J Vasc Surg. 1997;26(3):517-38.

18. Aslam F, Haque A, Foody J, et al. Peripheral arterial disease: current perspectives and new trends in management. South Med J. 2009;102(11):1141-9.

19. Hirsch AT, Criqui MH, Treat-Jacobson D, et al. Peripheral arterial disease detection, awareness, and treatment in primary care. JAMA. $2001 ; 286(11): 1317-24$.

20. Gardner AW, Poehlman ET. Exercise rehabilitation programs for the treatment of claudication pain. A meta-analysis. JAMA. 1995;274(12):975-80.

21. Hiatt WR, Wolfel EE, Meier $\mathrm{RH}$, et al. Superiority of treadmill walking exercise versus strength training for patients with peripheral arterial disease. Implications for the mechanism of the training response. Circulation. 1994;90(4): 1866-74.

22. Lawall H, Bramlage $P$, Amann B. Treatment of peripheral arterial disease using stem and progenitor cell therapy. J Vasc Surg. 2010.

23. Lawall H, Bramlage P, Amann B. Stem cell and progenitor cell therapy in peripheral artery disease. A critical appraisal. Thromb Haemost. 2010;103(4):696-709.

24. Lederman RJ, Mendelsohn FO, Anderson RD, et al. Therapeutic angiogenesis with recombinant fibroblast growth factor-2 for intermittent claudication (the TRAFFIC study): a randomised trial. Lancet. 2002;359(9323):2053-8.

25. Rajagopalan S, Mohler ER, 3rd, Lederman RJ, et al. Regional angiogenesis with vascular endothelial growth factor in peripheral arterial disease: a phase II randomized, double-blind, controlled study of adenoviral delivery of vascular endothelial growth factor 121 in patients with disabling intermittent claudication. Circulation. 2003;108(16):1933-8. 
26. Leng GC, Fowler B, Ernst E. Exercise for intermittent claudication. Cochrane Database Syst Rev. 2000(2):CD000990.

27. Markkanen JE, Rissanen TT, Kivela A, et al. Growth factor-induced therapeutic angiogenesis and arteriogenesis in the heart--gene therapy. Cardiovasc Res. 2005;65(3):656-64.

28. Tammela T, Enholm B, Alitalo K, et al. The biology of vascular endothelial growth factors. Cardiovasc Res. 2005;65(3):550-63.

29. Aghi M, Chiocca EA. Contribution of bone marrow-derived cells to blood vessels in ischemic tissues and tumors. Mol Ther. 2005;12(6):994-1005.

30. Rofsky NM, Adelman MA. MR angiography in the evaluation of atherosclerotic peripheral vascular disease. Radiology. 2000;214(2):325-38.

31. Menke J, Larsen J. Meta-analysis: Accuracy of contrast-enhanced magnetic resonance angiography for assessing steno-occlusions in peripheral arterial disease. Ann Intern Med. 2010;153(5):325-34.

32. Leiner T, Tordoir JH, Kessels AG, et al. Comparison of treatment plans for peripheral arterial disease made with multi-station contrast mediumenhanced magnetic resonance angiography and duplex ultrasound scanning. J Vasc Surg. 2003;37(6):1255-62.

33. de Vries M, de Koning PJ, de Haan MW, et al. Accuracy of semiautomated analysis of 3D contrast-enhanced magnetic resonance angiography for detection and quantification of aortoiliac stenoses. Invest Radiol. 2005;40(8):495-503.

34. Jakobs TF, Wintersperger BJ, Becker CR. MDCT-imaging of peripheral arterial disease. Semin Ultrasound CT MR. 2004;25(2):145-55.

35. Mui KW, Zeebregts $\mathrm{CJ}$, van den Hout $\mathrm{H}$, et al. Impact of incidental renal artery stenosis on long-term mortality in patients with peripheral arterial disease undergoing vascular procedure. J Vasc Surg. 2011;54(3):785-90.

36. de Vries M, Nijenhuis RJ, Hoogeveen RM, et al. Contrast-enhanced peripheral MR angiography using SENSE in multiple stations: feasibility study. J Magn Reson Imaging. 2005;21(1):37-45.

37. Leiner T, Kessels AG, Nelemans PJ, et al. Peripheral arterial disease: comparison of color duplex US and contrast-enhanced MR angiography for diagnosis. Radiology. 2005;235(2):699-708.

38. Giovagnoni A, Catalano C. Application of blood-pool agents in visualization of peripheral vessels. Eur Radiol. 2007;1 7 Suppl 2:B1 8-23.

39. Kos S, Reisinger C, Aschwanden $M$, et al. Pedal angiography in peripheral arterial occlusive disease: first-pass i.v. contrast-enhanced MR angiography with blood pool contrast medium versus intraarterial digital subtraction angiography. AJR Am J Roentgenol. 2009;192(3):775-84.

40. Hadizadeh DR, Gieseke J, Lohmaier SH, et al. Peripheral MR angiography with blood pool contrast agent: prospective intraindividual comparative study of high-spatial-resolution steady-state MR angiography versus standard-resolution first-pass MR angiography and DSA. Radiology. 2008;249(2):701-11. 
41. Grijalba FU, Esandi MC. Comparison of gadofosveset-enhanced threedimensional magnetic resonance angiography with digital subtraction angiography for lower-extremity peripheral arterial occlusive disease. Acta Radiol. 2010;51(3):284-9.

42. Nielsen YW, Eiberg JP, Logager VB, et al. Whole-body magnetic resonance angiography with additional steady-state acquisition of the infragenicular arteries in patients with peripheral arterial disease. Cardiovasc Intervent Radiol. 2010;33(3):484-91.

43. Thompson RB, Aviles RJ, Faranesh AZ, et al. Measurement of skeletal muscle perfusion during postischemic reactive hyperemia using contrastenhanced MRI with a step-input function. Magn Reson Med. 2005;54(2):289-98.

44. Mohajer K, Zhang H, Gurell D, et al. Superficial femoral artery occlusive disease severity correlates with MR cine phase-contrast flow measurements. J Magn Reson Imaging. 2006;23(3):355-60.

45. Ledermann HP, Schulte AC, Heidecker HG, et al. Blood oxygenation leveldependent magnetic resonance imaging of the skeletal muscle in patients with peripheral arterial occlusive disease. Circulation. 2006;113(25):2929-35.

46. Voth $\mathrm{M}$, Haneder S, Huck K, et al. Peripheral magnetic resonance angiography with continuous table movement in combination with high spatial and temporal resolution time-resolved MRA With a total single dose $(0.1$ $\mathrm{mmol} / \mathrm{kg}$ ) of gadobutrol at 3.0 T. Invest Radiol. 2009;44(9):627-33.

47. Meyer RA, Foley JM, Harkema SJ, et al. Magnetic resonance measurement of blood flow in peripheral vessels after acute exercise. Magn Reson Imaging. 1993;11(8):1085-92.

48. Walker MF, Souza SP, Dumoulin CL. Quantitative flow measurement in phase contrast MR angiography. J Comput Assist Tomogr. 1988;12(2):304-13.

49. Barrett T, Brechbiel M, Bernardo M, et al. MRI of tumor angiogenesis.

J Magn Reson Imaging. 2007;26(2):235-49.

50. Isbell DC, Epstein FH, Zhong $X$, et al. Calf muscle perfusion at peak exercise in peripheral arterial disease: measurement by first-pass contrast-enhanced magnetic resonance imaging. J Magn Reson Imaging. 2007;25(5):1013-20.

51. Weber MA, Krix M, Delorme S. Quantitative evaluation of muscle perfusion with CEUS and with MR. Eur Radiol. 2007;17(10):2663-74.

52. Noseworthy MD, Bulte DP, Alfonsi J. BOLD magnetic resonance imaging of skeletal muscle. Semin Musculoskelet Radiol. 2003;7(4):307-15.

53. Noseworthy MD, Kim JK, Stainsby JA, et al. Tracking oxygen effects on MR signal in blood and skeletal muscle during hyperoxia exposure. J Magn Reson Imaging. 1999;9(6):814-20. 


\section{CHAPTER 1}

54. Prielmeier F, Merboldt KD, Hanicke W, et al. Dynamic high-resolution MR imaging of brain deoxygenation during transient anoxia in the anesthetized rat. J Cereb Blood Flow Metab. 1993;13(5):889-94.

55. Prielmeier F, Nagatomo Y, Frahm J. Cerebral blood oxygenation in rat brain during hypoxic hypoxia. Quantitative MRI of effective transverse relaxation rates. Magn Reson Med. 1994;31(6):678-81.

56. Donahue KM, Van Kylen J, Guven S, et al. Simultaneous gradientecho/spin-echo EPI of graded ischemia in human skeletal muscle. J Magn Reson Imaging. 1998;8(5):1106-13.

57. Jordan BF, Kimpalou JZ, Beghein N, et al. Contribution of oxygenation to BOLD contrast in exercising muscle. Magn Reson Med. 2004;52(2):391-6. 


\section{CHAPTER 2}

MRI in peripheral arterial disease:

Reproducibility of the assessment of

morphological and functional vascular status

B. Versluis, W.H. Backes, M.G.A. van Eupen, K. Jaspers, P.J. Nelemans, E.V. Rouwet, J.A.W. Teijink, W.P.Th.M. Mali, G.W.H. Schurink, J.E. Wildberger, T. Leiner

Published in Investagive Radiology, 2011 :

Versluis B, Backes WH, van Eupen MG, et al. Magnetic resonance imaging in peripheral arterial disease: reproducibility of the assessment of morphological and functional vascular status. Invest Radiol.

$2011 ; 46(1): 11-24$ 


\section{CHAPTER 2}

\section{Abstract}

\section{Objectives}

The aim of the current study was to test the reproducibility of different quantitative MRI methods to assess the morphological and functional peripheral vascular status and vascular adaptations over time in patients with peripheral arterial disease (PAD).

\section{Materials and methods}

Ten patients with proven PAD (intermittent claudication) and arterial collateral formation within the upper leg and 10 healthy volunteers were included. All subjects underwent two identical MR examinations of the lower extremities on a clinical 1.5T MR system, with a time interval of at least 3 days. The MR protocol consisted of 3D contrast-enhanced MR angiography (CE-MRA) to quantify the number of arteries and artery diameters of the upper leg, 2D cine MR phase contrast angiography (PCA) flow measurements in the popliteal artery, dynamic contrastenhanced (DCE) perfusion imaging to determine the influx constant and area-under-curve and dynamic blood oxygen level-dependent (BOLD) imaging in calf muscle to measure maximal relative $\mathrm{T} 2 *$ changes and time-to-peak. Data were analyzed by two independent MRI readers. Interscan and interreader reproducibility were determined as outcome measures and expressed as the coefficient of variation (CV).

\section{Results}

Quantification of the number of arteries, artery diameter and blood flow proved highly reproducible in patients (CV 2.6, 4.5 and 15.8\% respectively at interscan level and 9.0, 8.2 and $7.0 \%$ at interreader level). Reproducibility of DCE and BOLD MRI was poor in patients with a CV up to $50.9 \%$. 


\section{Conclusion}

Quantification of the morphological vascular status by CE-MRA, as well as PCA MRI to assess macrovascular blood flow proved highly reproducible in both PAD patients and healthy volunteers and might therefore be helpful in studying the development of collateral arteries in PAD patients and in unraveling the mechanisms underlying this process. Functional assessment of the microvascular status using DCE and BOLD MRI did not prove reproducible at $1.5 \mathrm{~T}$ and is therefore currently not suitable for (clinical) application in PAD. 


\section{Introduction}

Peripheral arterial disease (PAD), characterized by arteriosclerotic stenosis or occlusion of pelvic and/or lower extremity arteries, may manifest clinically as intermittent claudication or critical limb ischemia (1). The primary treatment for patients with intermittent claudication is supervised exercise therapy, which may stimulate collateral blood artery formation to increase muscle perfusion (1-3). Stimulation of collateral blood vessel growth is also the main target of angiogenic growth factor or stem cell administration to improve tissue oxygenation in patients with critical limb ischemia (4-6). The exact mechanisms underlying this process are presently unknown, but are thought to involve both adaptation of existing arteries and the formation of new collateral arteries over time (7). Furthermore, it is presently unknown to what extent different therapeutic options contribute to these vascular adaptive responses, and to what extent functional improvement of patients is related to such vascular adaptations $(1,8)$.

Uncertainties concerning the exact type and extent of vascular adaptations in humans are partly due to the lack of non-invasive diagnostic methods to visualize and quantify these processes. Quantitative noninvasive imaging tools that can be used to study the process of vascular adaptation and to monitor the effectiveness of vessel-stimulating therapies are therefore highly desirable. Magnetic resonance imaging (MRI) techniques are attractive for this purpose, as they provide high spatial resolution to visualize the entire peripheral vascular tree, including small collateral arteries. Furthermore, MRI techniques are suitable to obtain information on vascular morphology and function and allow repeated measurements without ionizing radiation exposure (9-15). However, for evaluation of vascular responses over time it is crucial that MRI provides reproducible quantitative measures.

The aim of the current study was to test the reproducibility of different MRI methods to assess the morphological and functional vascular status and adaptations of the peripheral vasculature over time. Morphologically, the reproducibility was determined of a method to quantify the 
number of arteries in the upper leg, visualized by contrast-enhanced MR angiography (CE-MRA). Development of collateral arteries might result in an increased blood supply to and subsequent enlargement of the collateral re-entrance zone. This collateral re-entrance zone represents the level at which collaterals re-enter the large native conduit artery distal to an occluded segment of the arterial tree (distal superficial femoral or popliteal artery in our study), For this, the reproducibility of diameter measurements of the distal conduit artery was also assessed. Functional vascular status was determined both at macro- and microvascular level. Reproducibility of macrovascular function was studied by measuring peak arterial blood flow in the popliteal artery (PA) with quantitative 2D cine MR phase contrast angiography (PCA) (10, 16-19). Reproducibility of microvascular function was determined in the calf musculature by perfusion measurements using dynamic contrastenhanced (DCE) MRI $(9,20,21)$ as well as the blood oxygenation measurements using dynamic blood oxygen level-dependent (BOLD) MRI (13, 22-27). All measurements were performed in a group of PAD patients with proven extensive collateral artery formation and a group of healthy volunteers.

\section{Materials and methods}

\section{Phantom study}

A vessel phantom was used to determine the diameter of the smallest arteries that can be detected with our clinically used CE-MRA protocol. This phantom consisted of ten tubes with varying inner diameters: $6.00,2.03,1.57,1.40,1.01,0.76,0.51,0.25,0.18$ and $0.13 \mathrm{~mm}$. These tubes were filled with the same gadolinium-based contrast agent as used in the patient study (gadopentetate dimeglumine, (Magnevist), Bayer HealthCare Pharmaceuticals, Berlin, Germany), with a concentration of $11 \mathrm{mmol} / \mathrm{L}$, which is representative of the peak arterial concentration in our human study. Tubes were immersed in a muscle tissue mimicking fluid $(\mathrm{Tl}=800 \mathrm{~ms}, 0.2 \mathrm{mmol} / \mathrm{L}$ gadopentetate dimeglumine solution). 


\section{Chapter 2}

MRI was performed using the same acquisition protocol as used in the human studies detailed below. Scan parameters are given in table 1 . Acquired voxel sizes were $1.22 \times 1.52 \times 2.4 \mathrm{~mm}$. Subsequently, voxels were interpolated to $0.92 \times 0.92 \times 1.2 \mathrm{~mm}$ during reconstruction by zero-filling. The phantom was positioned slightly angulated with respect to the $z$-axis of the MRI scanner to average the signal over many subvoxel positions.

For image analysis, a line segment perpendicularly intersecting the depicted tubes was manually drawn. The intensity profile along this line was analyzed to determine the smallest detectable tube diameter by counting the number of peaks in the signal intensity profile.

\section{Human study}

Over a period of 6 months, 10 patients (mean age $64.7 \pm 6.1$ years; range 57 to 73 years; 9 men and 1 woman) with proven PAD (intermittent claudication, Fontaine stage II, Rutherford stage 1-3) and arterial collateral vessel formation within the upper leg and 10 healthy volunteers without signs and symptoms of PAD (mean age $26.7 \pm 11.5$ years; range 18 to 50 years; 2 men and 8 women) were included. PAD patients were eligible for the study if peripheral CE-MRA examinations as part of routine diagnostic work-up confirmed the presence of superficial femoral artery (SFA) occlusion with a collateral arterial network in the upper leg. Patients with contra-indications to MRA, including claustrophobia, known gadolinium based contrast agent allergy, and low estimated glomerular filtration rate $\left(<30 \mathrm{~mL} / \mathrm{kg} / 1.73 \mathrm{~m}^{2}\right)$ were excluded from participation. The study was approved by the institutional medical ethics committee and all patients and healthy controls gave written informed consent before inclusion. 
REPRODUCIBILITY OF MORPHOLOGIC AND FUNCTIONAL VASCULAR MRI

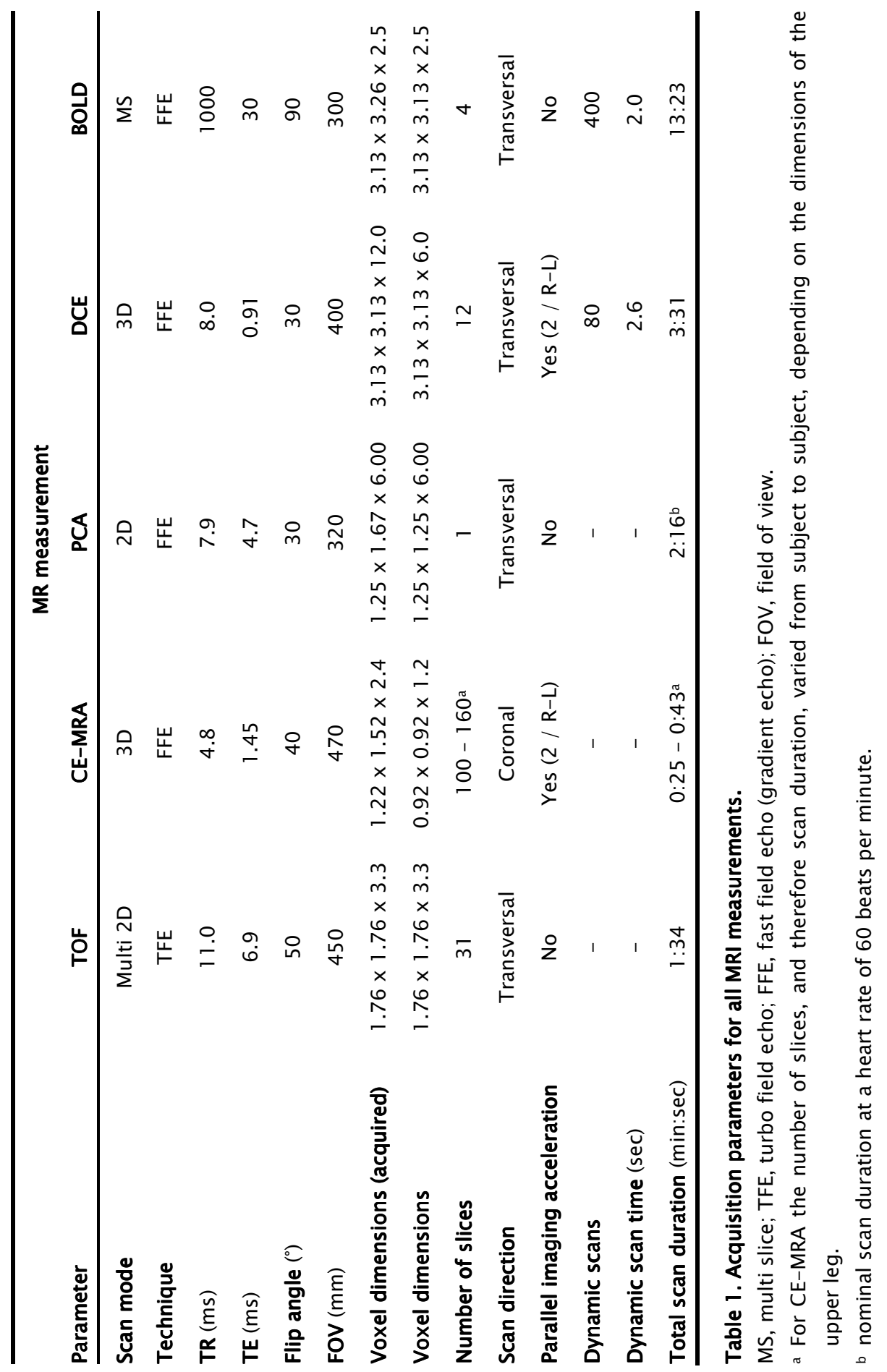



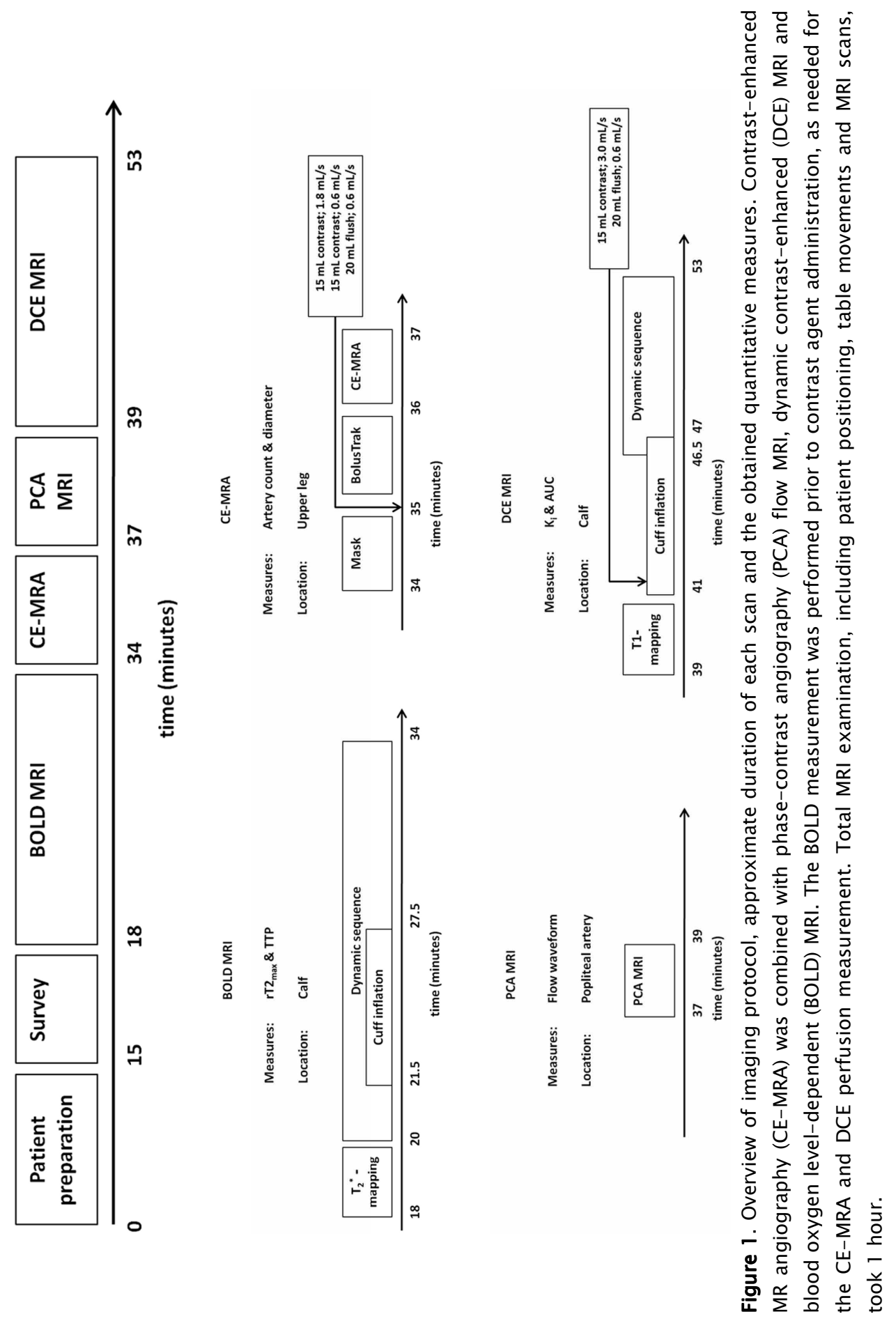


\section{Magnetic Resonance Imaging}

All subjects underwent two MRI examinations on different days (mean interval 14.6 days, range 3 - 68 days). An overview of the imaging protocol is given in figure 1 . The entire examination lasted one hour. Patients received no treatment between both examinations. All examinations were performed on a 1.5-T commercially available system (Intera, Philips Medical Systems, Best, The Netherlands). For signal reception we used a dedicated 12-element phased-array peripheral vascular coil with a craniocaudal coverage of $128 \mathrm{~cm}$ (Philips Medical Systems, Best, The Netherlands). Subjects were imaged in the supine position and care was taken not to deform calf muscle by calf-bed contact. All subjects were lying in this position for at least 15 minutes before the first measurement was started. Scan parameters for all acquisitions are given in table 1.

Contrast. For CE-MRA and DCE MRI a dose of respectively 30 and $15 \mathrm{~mL}$ gadopentetate dimeglumine (Magnevist, Bayer HealthCare Pharmaceuticals, Berlin, Germany) was injected intravenously as a single biphasic bolus in the cubital vein, using a remote controlled injection system (Medrad Spectris, Indianola, PA). Although CE-MRA for this study comprised only one station (upper leg), an injection of $30 \mathrm{~mL}$ of contrast agent was chosen for best comparison of image quality with common clinical three-station peripheral CE-MRA, for which we normally administer a single bolus of $30 \mathrm{~mL}$. After contrast medium administration, 20 $\mathrm{mL}$ of saline was injected. Real time bolus monitoring software (BolusTrak, Philips Medical Systems, Best, The Netherlands) was used to visualize the arrival of the bolus in the proximal SFA with a refresh rate of proximally 1 frame/sec. Upon first sight of contrast arrival in the proximal SFA, image acquisition for the CE-MRA sequence was started. For DCE MRI, cuff inflation (see below) was immediately followed by a single bolus of $15 \mathrm{~mL}$ contrast agent, allowing systemic contrast equilibration in the arterial blood pool proximal to the occlusion during cuff compression. 


\section{CHAPTER 2}

Cuff paradigm. For DCE and BOLD MRI, postischemic reactive hyperemia was provoked in both lower legs using inflatable cuffs (Medrad, Indianola, PA). Reactive hyperemia was provoked to ensure measurable signal alterations in DCE and BOLD MRI and to ensure a most reproducible state of vasodilatation for both examinations for each patient (13). A cuff paradigm was chosen, as this method was believed to be more reproducible and suitable for MRI in comparison with muscle exercise and less invasive in comparison with drug induced vasodilation. Besides, a cuff paradigm ensures a vascular step-input function of contrast agent, as preferred for DCE MRI (9). The cuffs were placed at mid-thigh level and manually inflated to suprasystolic values $(>50 \mathrm{mmHg}$ above brachial systolic blood pressure) during 6 minutes prior to BOLD and DCE MRI, ensuring total arterial occlusion (13).

Survey. A non-enhanced time-of-flight (TOF) scan of the pelvic, upper and lower leg station was acquired to prescribe the imaging volumes of interest for morphological and functional imaging. A turbo field echo (TFE) pulse sequence was used with a $180^{\circ}$ inversion prepulse to suppress stationary tissues. Thirty-one axial slices per station were acquired with 3.3-mm slice thickness and 11-mm interslice gap, and an inferiorly concatenated saturation band. The standard quadrature body coil was used for signal transmission and reception. For positioning of the 3D CE-MRA volumes maximum intensity projections (MIP) were generated in 3 orthogonal directions.

CE-MRA. An one-station 3D CE-MRA was performed with voxel sizes identical of the phantom study. Whereas clinical CE-MRA examinations are usually limited to visualization of a thin anteroposterior imaging volume only including the large conduit arteries in the upper leg (i.e. SFA and PA), for this study the entire muscular volume of the upper leg was imaged to visualize all the arteries including any collateral branches within the upper leg. Prior to contrast medium administration, a nonenhanced 'mask' image data set was acquired with exactly the same acquisition parameters as the CE-MRA, enabling background tissue suppression by image subtraction. 
PCA MRI. Blood flow data were obtained in the PA (distal to the vascular lesion in the SFA in patients). A single slice was positioned perpendicular to the course of the PA. Twenty cardiac phases were acquired over the $R-R$ interval with an encoding velocity (venc) of 100 $\mathrm{cm} / \mathrm{s}$ in the craniocaudal direction. VCG-tracing was used for retrospective cardiac synchronization. The nominal duration of this acquisition was 136 seconds at a heart rate of 60 beats per minute.

DCE MRI. DCE-MRI was preceded by T1 mapping to determine pre-contrast $\mathrm{Tl}$ relaxation times before contrast enhancement, using a scan sequence with identical contrast parameters and geometry as for the subsequent DCE acquisitions, but with variable flip angles $(2,5,10$, 15,25 and $\left.35^{\circ}\right)(9,28)$. A dynamic 3D T1-weighted gradient echo sequence was used for DCE MRI (table 1). Slices were positioned at the maximum diameter of the calf. Five dummy scans were acquired before the actual measurement to avoid varying $\mathrm{Tl}$ saturation effects. The acquisition started 330 seconds after cuff inflation. The cuff was then rapidly deflated (within 2 seconds) together with the start of the $6^{\text {th }}$ dynamic scan.

BOLD MRI. For BOLD imaging, a multi-shot single-echo echo planar imaging (EPI) sequence with fat suppression (spectral presaturation with inversion recovery, SPIR) was used to detect the T2* responses to oxyhemoglobin changes. Slices were also positioned at the maximum diameter of the calf. The first 1:30 minutes of the measurement were used to obtain baseline values. The cuff was then inflated for 6 minutes, while the measurement continued (data not used for analysis) and after cuff deflation the measurement continued for approximately 6 minutes. The dynamic study was preceded by a series of EPI sequences to determine the $T 2$ * relaxation time of muscle tissue, using the same parameters and geometry but increasing echo times of 10, 15, 20,30, 40 and $50 \mathrm{~ms}$. 


\section{CHAPTER 2}

\section{Image analysis}

In patients the most symptomatic leg was analyzed for all measurements. In healthy volunteers reproducibility measures were determined for both legs separately and these measures were subsequently averaged. Two independent MRI readers (ME and BV) analyzed all datasets, blinded for each other's results and the order of the examinations.

\section{Vascular morphology}

$C E-M R A$. Image analysis was performed on a dedicated image post processing workstation (MacPro, running OS X, version 10.5.4, Apple Inc., Cupertino, CA). Both source images and subtracted images were analyzed to ensure that no collateral arteries were missed due to misregistration artifacts between the source and mask images.

The MRI readers manually quantified the number of collaterals by counting the total number of arteries crossing transverse planes in the upper leg at five different levels. In patients, arteries were counted at

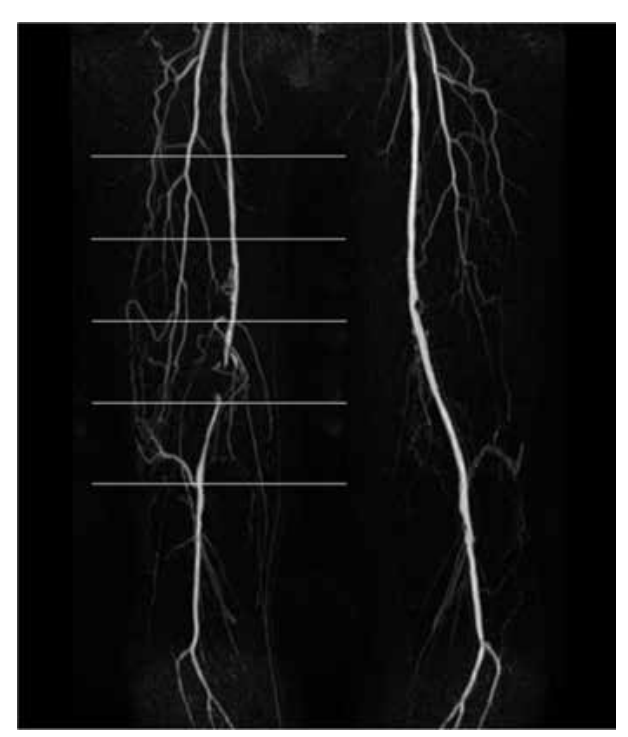

Figure 2. Maximum intensity projection (MIP) after background subtraction of a CEMRA in a PAD patient with segmental occlusion of the superficial femoral artery with extensive collateral blood vessel formation. The image shows the location of the five transverse planes, represented by the five horizontal lines, at which quantification of the number of arteries was manually performed. This MIP image is used to demonstrate the locations of the measurements; the actual measurements were performed using the $3 \mathrm{D}$ image data set. 
the following levels: $5 \mathrm{~cm}$ below, exactly at and 5,10 , and $15 \mathrm{~cm}$ above the location where the SFA was reconstituted distal to the occlusion (figure 2). In volunteers these planes were located at 10, 15, 20, 25 and $30 \mathrm{~cm}$ above the distal margin of the femoral condyls, as this corresponded best to the levels used in patients. In volunteers both legs were analyzed, whereas in patients only the most symptomatic leg (i.e. with clinically significant vascular pathology) was analyzed.

Arteries were counted using the open source DICOM viewer OsiriX (OsiriX, Geneva, Switzerland, version 3.5.1, http://www.osirixviewer.com/), using the original source images as well as coronal and sagittal multiplanar reconstructions (MPR). Identification of the correct counting plane was ensured by placing a crosshair at the anatomical landmarks as described above. Subsequently, the total number of arteries intersecting the five planes was determined, including conduit arteries (SFA or PA), terminal muscle branches and any collateral arteries. Arteries were distinguished from veins by scrolling through source images and MPR stacks when it was necessary to find an upstream connection to a named artery.

Diameter of the collateral re-entrance zone and image quality, in terms of contrast-to-noise ratio (CNR), were assessed on source images. Artery diameter and CNR were determined below the vascular lesion at the level of the collateral re-entrance zone (distal SFA or proximal PA in PAD patients or at the level of the PA in healthy volunteers). Artery diameter was measured at the coronal source images by manually drawing a line segment perpendicular to the artery at the same location as the most inferior transverse plane, used for counting the arteries. Next, the intensity profile along this line was obtained to determine the full-width-at-half-maximum of the arterial lumen, which represented the arterial diameter. Image quality of vascular depiction was expressed as the contrast-to-noise ratio (CNR), which was defined as the difference between signal intensity within the vessel and a similarly sized region of interest directly adjacent to the vessel. 


\section{CHAPTER 2}

\section{Vascular function}

PCA MRI. Modulus and phase images were reconstructed from the PCA data. The image software application MRIcro (MRIcro, http://www.mricro.com/) was used to manually draw a region of interest (ROI) covering the entire visible surface of the PA on each of the 20 reconstructed modulus images spanning the $R-R$ interval. These regions of interest (ROI's) were analyzed, using self-written software code (Matlab, The Mathworks inc., Natick, MA) to obtain velocity and flow waveforms from the phase images. Reproducibility was determined for the peak flow (unit: $\mathrm{mL} / \mathrm{s}$ ), as flow has been shown to be a more reliable parameter compared to velocity and peak values have shown larger differences between patients and healthy volunteers and proven to be more reliable compared to mean values for small arteries like the PA (10).

DCE MRI. MRIcro was used to manually draw large ROl's covering the entire cross-section of the calf muscle (including vessels) in four successive slices. Special attention was given to draw ROl's of both scans for every subject at the same location.

Self-written Matlab software code was used for the analysis of $\mathrm{T}_{1}$ weighted signal intensity time-courses for each ROI. Individual signal time-courses were normalized with respect to resting values, measured prior to contrast agent arrival and converted to contrast agent concentration, using the relation between $T_{1}$ relaxation time and the signal intensity for a spoiled gradient echo pulse sequence. Signal to contrast agent concentration conversion was applied to correct for the presence of residual contrast agent from the CE-MRA, which may vary between patients and healthy volunteers. In addition, contrast concentration is less scan technique dependent compared to signal intensity.

A vascular input function could not be obtained in every patient. Therefore a predefined step-wise vascular input function (VIF) with a maximum (i.e. step size) concentration of $0.45 \mathrm{mM}$ was used for data analysis (9). The influx constant $\left(K_{i}\right.$, unit: $\left.\mathrm{min}^{-1}\right)$ and area-under-curve (AUC, unit: mMs) up to 90 seconds after contrast arrival were calculated 
as surrogate markers of microvascular blood flow (9). Reproducibility was determined for both $K_{i}$ and AUC.

BOLD MRI. Analogous to DCE MRI, a large ROI, covering the entire cross-section of the calf muscle was drawn in the 4 acquired slices to assess skeletal muscle oxygenation. Self-developed Matlab software code was used for analysis of the $\mathrm{T2}^{*}$ signal intensity time courses for each of the ROI's. Individual T2* time courses were divided by the average $\mathrm{T2}^{*}$ values measured prior to cuff inflation. The maximum relative $\mathrm{T}_{2}{ }^{*}$ change after cuff deflation relative to the resting condition value (rT2 $\max$, unit: \%) and TTP (unit: s) were determined (13). rT2 $\max$ and TTP were both used to determine the reproducibility of the BOLD technique.

\section{Statistical analysis}

To determine reproducibility, two agreement measures, being the coefficient of variation ( $\mathrm{CV}$ in \%) and the repeatability coefficient (RC), and a measure for reliability, the intra-class correlation coefficient (ICC) were determined. The CV was derived by dividing the overall mean within-subject standard deviation $\left(\mathrm{SD}_{\mathrm{ws}}\right)$ by the mean measurement value over all subjects. The RC gives the smallest noticeable difference that can be detected beyond measurement error and is defined as $1.96 \cdot \sqrt{ } 2 \cdot S_{\text {ws }}(29,30)$. The difference between 2 measurements in the same subject is expected to be less than the RC in $95 \%$ of the observations in cases where the measured quantity remains unchanged over time. A value of RC (or CV) lower than absolute (or relative) difference between mean values in patients and controls indicates good agreement. The ICC was calculated using a 1-way random model, according to ICC $=S_{b_{b s}}^{2} /\left(S_{b s}{ }^{2}+S D_{w s}{ }^{2}\right)$, where $S D_{b s}$ represents the standard deviation between subjects. The ICC is the fraction of total variance due to variation between subjects, rather than measurement error, and ranges between 0 and 1 . If the measurement error is small compared to the variation between subjects, the ICC approaches 1, i.e. reliability is very high.

Figure 3 schematically illustrates how interscan and interreader reproducibility were defined. Interscan reproducibility is influenced by 


\section{CHAPTER 2}

contrast injection, vascular opacification, positioning of the patient within the coil or magnet, preparation of the patient, physiological variances within the patient, and reader errors. Interscan reproducibility is mainly influenced by scan related inconsistencies and was calculated by averaging the results of both MRI readers for each scan (mean of MRI 1 for reader $A$ and $B$ versus the mean of MRI 2 for reader $A$ and $B$ in figure 3 ). Interreader reproducibility is an indicator of image reading error and is primarily influenced by reader experience. Interreader reproducibility was calculated by averaging the results of both scans for each MRI reader (mean of reader A for MRI 1 and MRI 2 versus the mean of reader $B$ for MRI 1 and MRI 2 in figure 3 ).

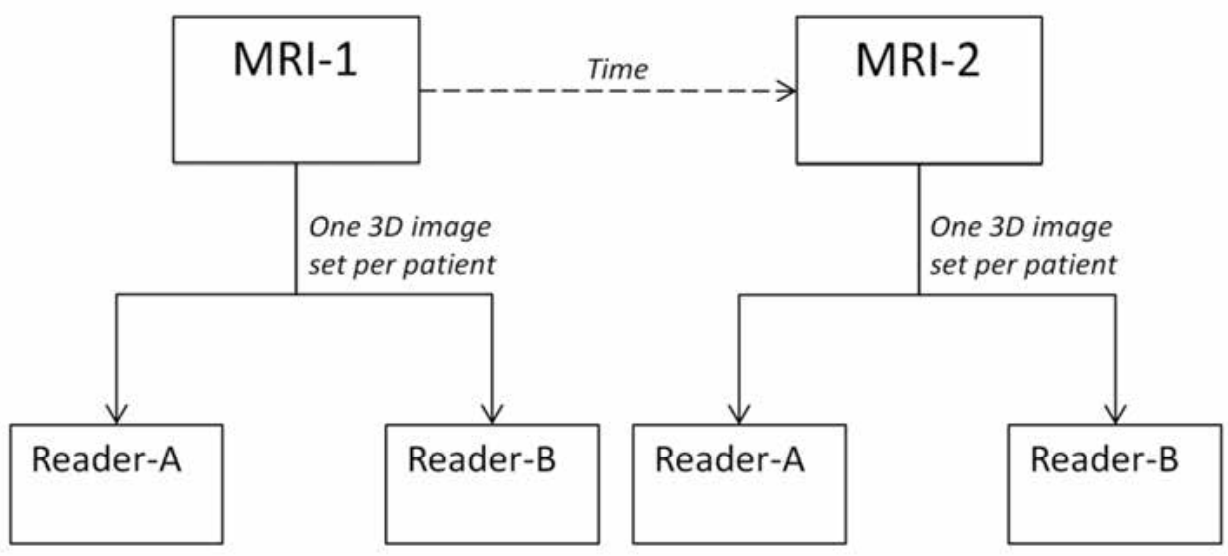

Figure 3. Schematic overview to define the interscan and interreader reproducibility.

Secondary to the reproducibility, an independent-samples $t$-test was performed to test the significance of differences in parameters between patients and healthy volunteers. $\mathrm{P}<0.05$ was considered statistically significant. All statistical analyses were performed with commercially available statistical software (SPSS 16.0, SPSS Inc., Chicago, IL). 


\section{Results}

\section{Vessel phantom}

Figure 4 displays a thin-slab maximum intensity projection (MIP) image of the vessel phantom. The smallest tube visualized had a diameter of $0.51 \mathrm{~mm}$. The smallest peak visible in the signal intensity profile matched the smallest tube visible on the MR angiogram.

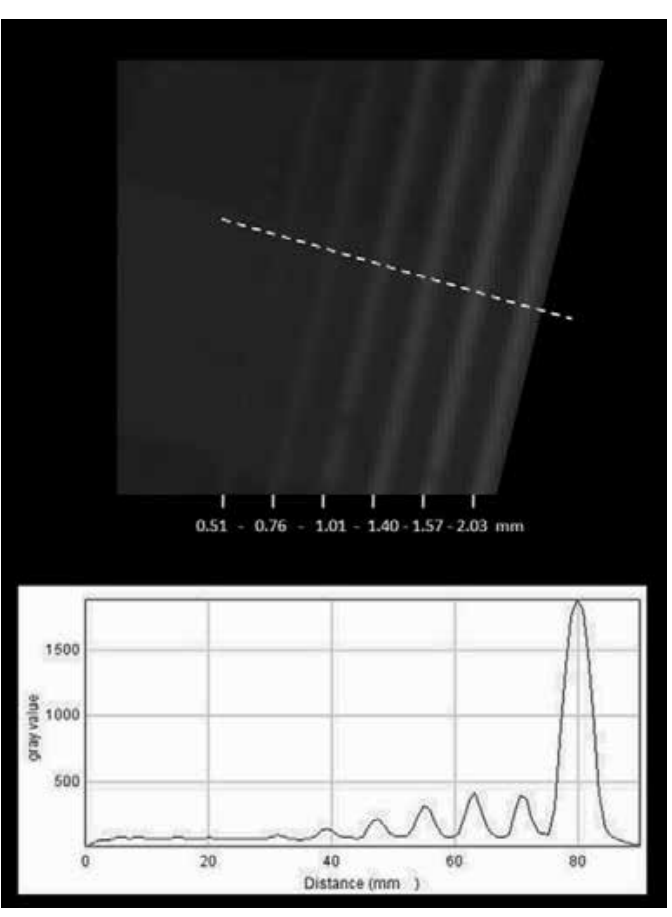

Figure 4. Thin slab (5 slices) maximum intensity projection (MIP) image showing the tubes with various diameters. Seven out of ten tubes can be distinguished. Graph shows the signal intensity profile measured at the level of the dotted line in the MIP image. The $6 \mathrm{~mm}$ tube with the highest signal intensity has been removed from the image to improve contrast.

\section{Subjects}

All patients and healthy volunteers underwent both MRI examinations without experiencing side effects or adverse events. In one patient there were severe subtraction misregistration artifacts in CE-MRA due to patient movement between acquisition of the mask and the contrastenhanced data sets. For this patient only the original contrast-enhanced images were analyzed. Reactive hyperemia could not be provoked in two out of ten patients as the cuff broke during cuff inflation. There were no side effects or adverse events in any of the study subjects. Severe discomfort due to cuff inflation was reported in 3 out of the 8 patients and 3 out of the 10 volunteers. 


\begin{tabular}{|c|c|c|c|c|c|}
\hline & & \multicolumn{2}{|c|}{ Patients } & \multicolumn{2}{|c|}{ Volunteers } \\
\hline & & $\begin{array}{l}\text { Source images } \\
\qquad(n=10)\end{array}$ & $\begin{array}{l}\text { Subtracted images } \\
\qquad(n=9)\end{array}$ & $\begin{array}{l}\text { Source images } \\
\qquad(n=10)\end{array}$ & $\begin{array}{l}\text { Subtracted images } \\
\qquad(n=10) \\
\end{array}$ \\
\hline Arteries $\mathrm{p}$ & plane & $15.7 \pm 3.5$ & $14.5 \pm 3.2^{\mathrm{a}}$ & $12.9 \pm 2.5$ & $13.1 \pm 2.3 \mathrm{~b}$ \\
\hline \multicolumn{6}{|c|}{ Interscan reproducibility } \\
\hline $\mathrm{CV}$ & $\%$ & 2.6 & 6.1 & 2.8 & 3.1 \\
\hline $\mathrm{RC}$ & & 1.1 & 2.5 & 1.0 & 1.1 \\
\hline ICC & $(95 \% \mathrm{Cl})$ & $0.99(0.95-0.99)$ & $0.92(0.75-0.98)$ & $0.98(0.90-0.99)$ & $0.97(0.89-0.99)$ \\
\hline \multicolumn{6}{|c|}{ Interreader reproducibility } \\
\hline CV & $\%$ & 9.0 & 10.1 & 11.1 & 10.0 \\
\hline $\mathrm{RC}$ & & 3.9 & 4.1 & 3.9 & 4.5 \\
\hline ICC & $(95 \% \mathrm{Cl})$ & $0.85(0.56-0.96)$ & $0.81(0.44-0.95)$ & $0.73(0.12-0.95)$ & $0.74(0.18-0.94)$ \\
\hline
\end{tabular}

Table 2. Numbers of arteries per plane and reproducibility of artery count.

Number of arteries is expressed as mean \pm SD. CV, coefficient of variance; RC, repeatability coefficient; ICC, intraclass correlation coefficient; $95 \% \mathrm{Cl}, 95 \%$ confidence interval. a $\mathrm{p}=$ $0.19,{ }^{b} \mathrm{p}=0.43$.

\section{Vascular morphology}

Artery count. Table 2 lists the number of arteries and reproducibility measures for source and subtracted images in patients and volunteers. Interscan and interreader reproducibility were better for source images compared to subtracted images for both groups. Furthermore, RC at interscan level was roughly 4 times lower than the RC at interreader level, indicating better agreement at interscan level. There were no relevant differences in reproducibility measures between patients and volunteers, both at interscan and interreader level. In figure 5 Bland-Altman plots are shown for artery count on CE-MRA source images for interscan and interreader variations (31). Both for patients and volunteers, the range between the $95 \%$ limits of agreement is much narrower, and therefore better, at interscan level compared to interreader level.

No significant differences were found in mean number of arteries per plane between the source and subtracted images in both patients 
and volunteers. The number of arteries per plane was significantly higher in patients compared to volunteers for CE-MRA $(p<0.05)$.
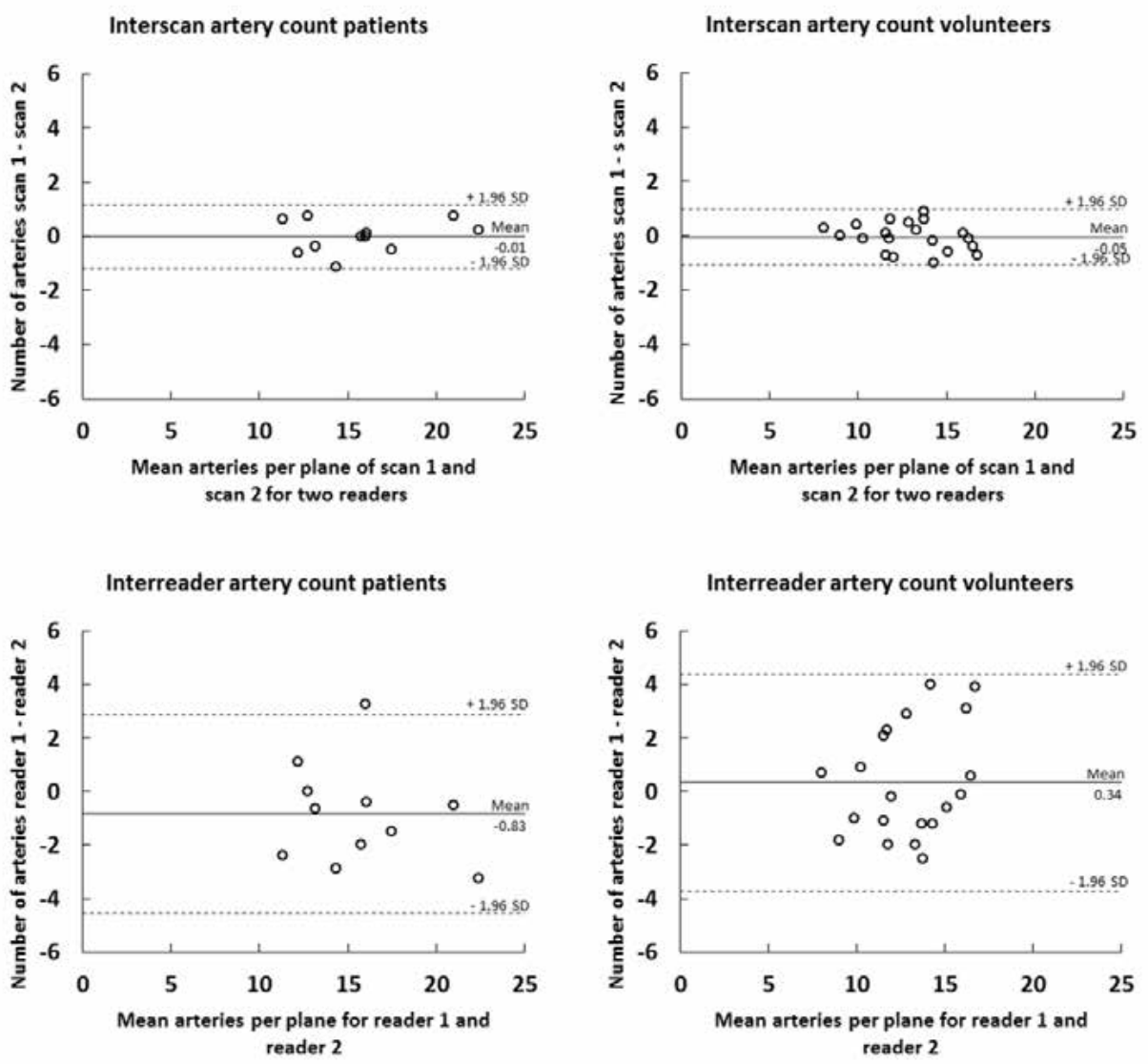

Figure 5. Interscan and interreader reproducibility of number of arteries per plane in PAD patients and volunteers. Bland-Altman plots showing difference in number of arteries between scans (interscan) or readers (interreader) (y-axis) against mean number of arteries ( $\mathrm{X}$-axis). Absolute mean difference is represented by the solid line and $95 \%$ confidence interval is given by the two dashed lines.

Artery diameter. In table 3 results of the arterial diameters of the collateral re-entrance zone in patients and PA in volunteers are listed. Analogous to the artery count, the interscan RC in patients was better than interreader RC, whereas in volunteers the reverse was found. This was the case true for the CV in patients and volunteers. ICC was low in 


\section{CHAPTER 2}

volunteers with respect to interscan level reproducibility. In figure 6 Bland-Altman plots are shown for the collateral re-entrance zone on source images at interscan and interreader level for both patients and healthy volunteers (31). The $95 \%$ limits of agreement show no large differences between interscan and interreader diameters for both patients as well as volunteers.

Interscan re-entrance zone diameter patients

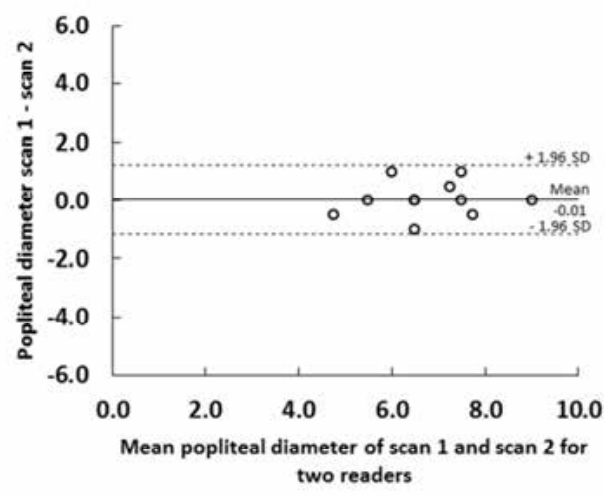

Interreader re-entrance zone diameter patients

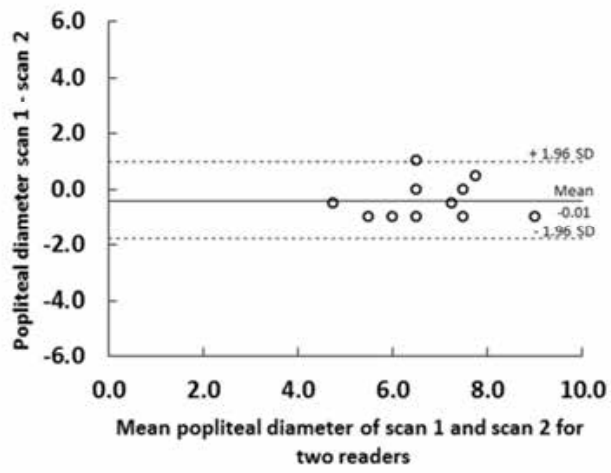

Interscan popliteal diameter volunteers

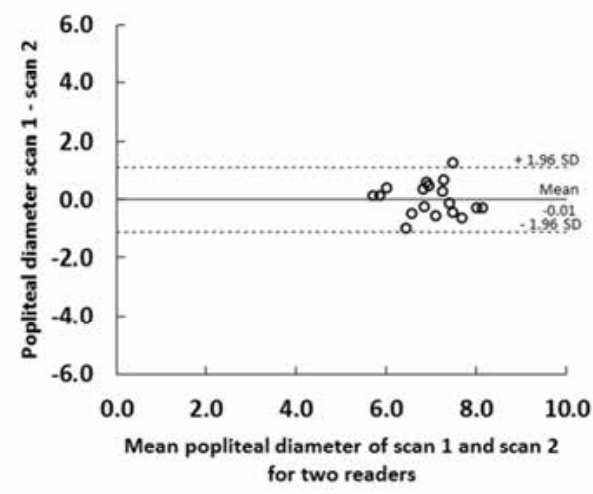

Interreader popliteal diameter volunteers

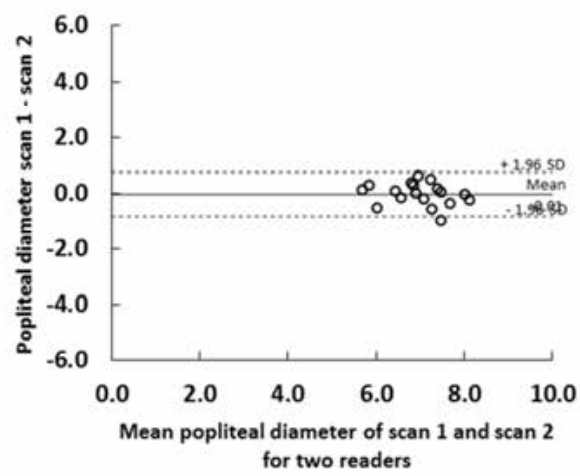

Figure 6. Interscan and interreader reproducibility of diameters of the conduit artery near the collateral re-entrance zone in PAD patients and of the popliteal artery in volunteers. Bland-Altman plots showing difference in diameter between scans (interscan) or readers (interreader) (y-axis) against mean vessel diameter ( $x$-axis). Absolute mean difference is represented by the solid line and $95 \%$ confidence interval is given by the two dashed lines. 


\begin{tabular}{cccc}
\hline & & $\begin{array}{c}\text { Patients } \\
(n=10)\end{array}$ & $\begin{array}{c}\text { Volunteers } \\
(n=10)\end{array}$ \\
\hline $\begin{array}{c}\text { Arterial diameter } \\
\text { Interscan reproducibility }\end{array}$ & $\mathrm{mm}$ & $6.9 \pm 1.2$ & $7.0 \pm 0.7 \mathrm{a}$ \\
CV & $\%$ & 4.5 & 5.6 \\
RC & $\mathrm{mm}$ & 0.8 & 1.1 \\
ICC & $(95 \% \mathrm{Cl})$ & $0.94(0.79-0.98)$ & $0.71(0.39-0.88)$
\end{tabular}

Interreader reproducibility

$\begin{array}{lccc}\text { CV } & \% & 8.2 & 4.0 \\ \text { RC } & \mathrm{mm} & 1.5 & 0.8 \\ \text { ICC } & (95 \% \mathrm{Cl}) & 0.79(0.44-0.94) & 0.84(0.63-0.94) \\ & & 64 \pm 31 & 84 \pm 31^{\mathrm{b}}\end{array}$

CNR

Table 3. Artery diameter, reproducibility and contrast-to-noise ratio.

Arterial diameter is expressed as mean \pm SD. CNR, contrast-to-noise ratio. a $p=0.43$, b $p$ $<0.05$.

\begin{tabular}{cccc}
\hline & & Patients & Volunteers \\
& & & \\
& & $(n=10)$ & $(n=10)$ \\
\hline Peak flow & $\mathrm{mL} / \mathrm{s}$ & $3.15 \pm 1.40$ & $9.77 \pm 2.06 \mathrm{a}$ \\
Interscan reproducibility & & \\
$\mathrm{CV}$ & $\%$ & 15.8 & 6.8 \\
$\mathrm{RC}$ & $\mathrm{mL} / \mathrm{s}$ & 1.4 & 1.9 \\
$\mathrm{ICC}$ & $(95 \% \mathrm{Cl})$ & $0.94(0.76-0.98)$ & $0.89(0.77-0.97)$ \\
$\mathrm{CV}$ & $\%$ & & 5.9 \\
$\mathrm{RC}$ & $\mathrm{mL} / \mathrm{s}$ & 7.0 & 1.6 \\
ICC & $(95 \% \mathrm{Cl})$ & $0.98(0.95-0.99)$ & $0.92(0.51-0.93)$ \\
\hline
\end{tabular}

Table 4. Reproducibility of PCA MRI in the popliteal artery.

Values are presented as mean \pm SD. ap $<0.01$.

No significant differences were found in artery diameters between patients and volunteers $(p=0.43)$. 


\section{CHAPTER 2}

Image quality. CNR values of the CE-MRA images are listed in table 3 as well. Patients showed a significantly lower CNR compared to volunteers $(p<0.05)$.

\section{Vascular function}

PCA MRI. Examples of measured waveforms are shown in figure 7. In table 4 the results of the reproducibility of the flow assessment are listed. In general, reproducibility of peak flow was good according to all different measures of agreement and reliability, with an interscan CV in patients up to $15.8 \%$. Interreader reproducibility was better than the interscan reproducibility, especially for the patient group.

Flow waveforms in patients were either bi- $(n=3)$ or monophasic $(n=7)$, whereas triphasic flow waveforms were obtained in all volunteers (figure 7). The peak flow in the PA of patients was less than $1 / 3$ compared to peak flow of healthy volunteers $(p<0.01)$. For PCA MRI, there was no relevant difference in any of the reproducibility measures between patients and healthy volunteers.
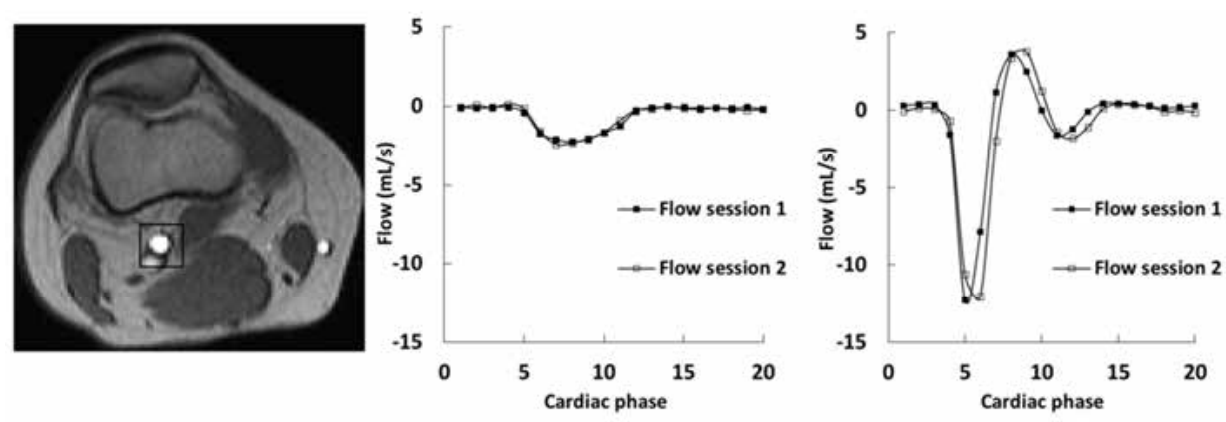

Figure 7. PCA modulus image (left panel) of the knee in a 68-year old patient suffering from Fontaine stage II PAD with a black square marking the popliteal artery. Typical flow waveforms of the popliteal artery are shown in a patient (center panel) and 24-year old healthy volunteer (right panel) at two different time points show excellent reproducibility over time. Note loss of tri-phasic flow waveform and a decrease in peak flow in the patient with PAD compared to the healthy volunteer. 

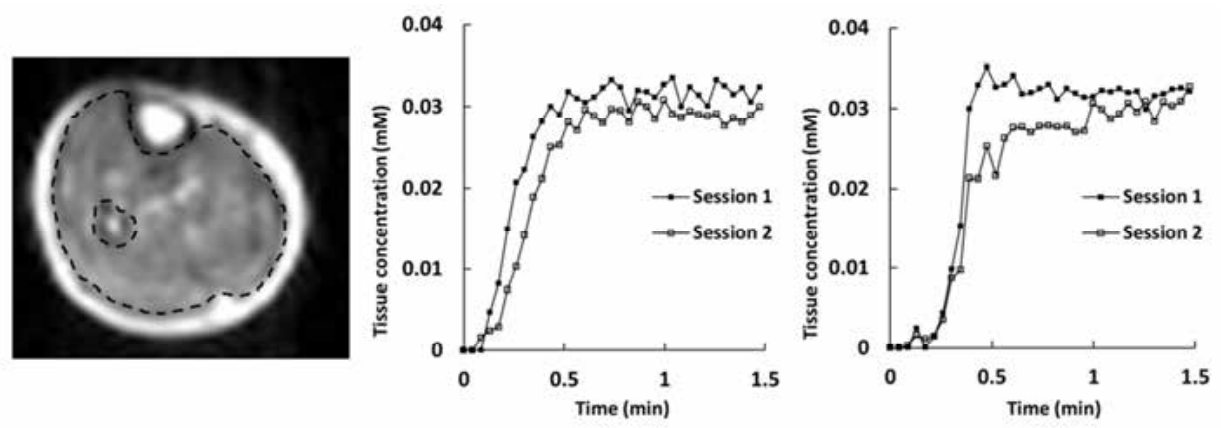

Figure 8. Example of DCE image (left panel) in a 68-year old patient suffering from Fontaine stage II PAD. Reproducibility was determined for a ROI covering the entire muscular volume (area between the black dotted contours). Graphics show the signal intensity converted to contrast agent concentration over time directly after cuff deflation in a PAD patient (center panel) and healthy volunteer (right panel) for both sessions. Note the interscan variability in PAD patients and healthy volunteers.
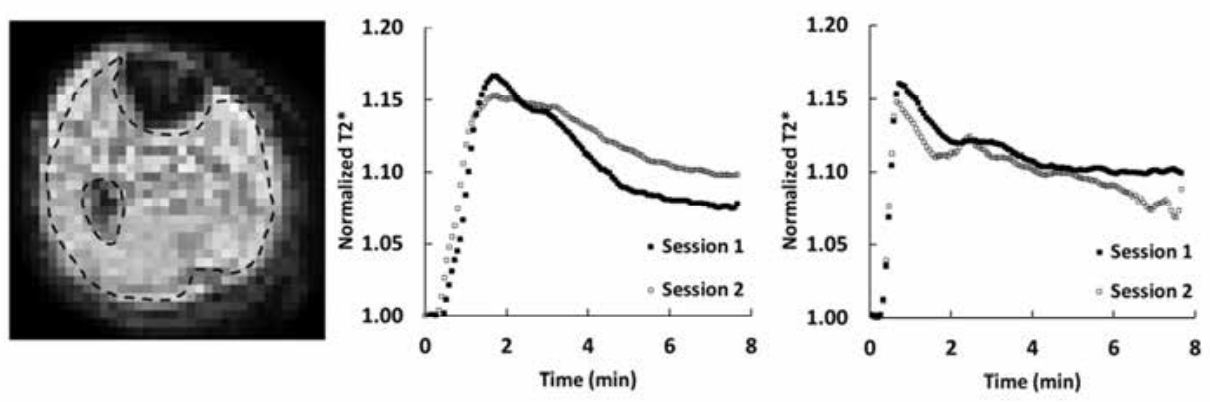

Figure 9. Example of BOLD image (left panel) in a 68-year old patient with Fontaine stage II PAD. Reproducibility was determined for a ROI covering the entire muscular volume (area between the black dotted contours). Graphics show the normalized $\mathrm{T}_{2}{ }^{*}$ over time directly after cuff deflation in a PAD patient (center panel) and healthy volunteer (right panel) for both sessions. Note the large interscan variability in PAD patients (center panel) and healthy volunteers (right panel).

DCE MRI. Examples of DCE MRI derived time courses are shown in figure 8. DCE-MRI interscan reproducibility was poor in patients for both 


\section{Chapter 2}

$K_{i}$ and AUC with interscan CV values up to $51 \%$ (table 5). Interreader reproducibility, on the contrary, was good with a low CV and RC, indicating a strong agreement between the readers, and a high ICC, indicating good interreader reliability. Interreader reproducibility was comparable between patients and healthy volunteers.

$K_{i}$ of the entire cross-section muscular volume showed a trend of lower perfusion values in the calf of patients compared to healthy volunteers ( $p=0.07$ ), whereas the AUC showed a trend of higher values in patients $(p=0.09)$.

\begin{tabular}{llcc}
\hline & & $\begin{array}{c}\text { Patients } \\
(\mathrm{n}=8)\end{array}$ & $\begin{array}{c}\text { Volunteers } \\
(\mathrm{n}=10)\end{array}$ \\
\hline$K_{i}$ & $\min ^{-1}$ & $0.64 \pm 0.22$ & $0.84 \pm 0.26^{\mathrm{a}}$ \\
$\begin{aligned} \text { Interscan reproducibility } \\
\text { CV }\end{aligned}$ & $\%$ & 50.9 & 17.7 \\
RC & $\min ^{-1}$ & 0.9 & 0.4 \\
ICC & $(95 \% \mathrm{Cl})$ & $0.00(0.00-0.65)$ & $0.71(0.22-0.92)$
\end{tabular}

Interreader reproducibility

\begin{tabular}{clccc} 
& CV & $\%$ & 6.3 & 3.5 \\
& RC & min-1 $^{-1}$ & 0.1 & 0.1 \\
& ICC & $(95 \% \mathrm{Cl})$ & $0.97(0.86-0.99)$ & $0.99(0.95-0.99)$ \\
\hline AUC & mMs & $4.18 \pm 1.26$ & $3.53 \pm 1.24 \mathrm{~b}$ \\
Interscan reproducibility & & & 10.7 \\
CV & $\%$ & 26.1 & 1.0 \\
RC & mMs & 3.0 & $0.69(0.17-0.91)$
\end{tabular}

Interreader reproducibility

\begin{tabular}{llcc} 
CV & $\%$ & 4.2 & 6.2 \\
RC & mMs & 0.5 & 0.6 \\
ICC & $95 \% \mathrm{Cl}$ & $0.98(0.92-0.99)$ & $0.97(0.89-0.99)$ \\
\hline
\end{tabular}

Table 5. Reproducibility of DCE MRI in the calf.

Values are presented as mean $\pm \mathrm{SD}$; $\mathrm{K}_{\mathrm{i}}$, influx constant; AUC, area under the curve; ap = 0.07 ; $b p=0.09$. 
BOLD MRI. Examples of BOLD time-courses are shown in figure 9. In patients BOLD derived parameters had interscan $C V$ values in $\mathrm{rT} 2 \max$ and TTP of up to $27 \%$ and $11 \%$ respectively and these results were comparable with those found in healthy volunteers (table 6). The interreader reproducibility was better in patients for both BOLD parameters and had CV values of up to $1.5 \%$ for rT2max and $11.3 \%$ for TTP (table 6).

TTP was delayed $(p<0.01)$ in the patient group compared to the volunteers, whereas the rT2max was nearly equal $(p=0.97)$.

\begin{tabular}{clcc}
\hline & & $\begin{array}{c}\text { Patients } \\
(\mathrm{n}=8)\end{array}$ & $\begin{array}{c}\text { Volunteers } \\
(\mathrm{n}=10)\end{array}$ \\
\hline rT2max & $\%$ & $13.2 \pm 6.4$ & $13.3 \pm 5.9 \mathrm{a}$ \\
Interscan reproducibility & & & \\
CV & $\%$ & 26.7 & 21.5 \\
RC & $\%$ & 9.7 & 7.9 \\
ICC & $(95 \% \mathrm{Cl})$ & $0.71(0.13-0.93)$ & $0.79(0.38-0.94)$
\end{tabular}

Interreader reproducibility

$\begin{array}{llcc}\text { CV } & \% & 1.5 & 4.3 \\ \text { RC } & \% & 0.5 & 1.6 \\ \text { ICC } & (95 \% \mathrm{Cl}) & 0.99(0.95-0.99) & 0.99(0.97-0.99)\end{array}$

TTP

$$
\mathrm{s}
$$$$
116 \pm 26.8
$$$$
60.7 \pm 15.9 \mathrm{~b}
$$

Interscan reproducibility

$\begin{array}{llcc}\text { CV } & \% & 10.6 & 15.9 \\ \text { RC } & \text { S } & 33.8 & 26.7 \\ \text { ICC } & (95 \% \mathrm{Cl}) & 0.80(0.35-0.96) & 0.69(0.18-0.91)\end{array}$

Interreader reproducibility

\begin{tabular}{llcc} 
CV & $\%$ & 11.3 & 18.8 \\
RC & S & 36.1 & 31.4 \\
ICC & $(95 \% \mathrm{Cl})$ & $0.78(0.28-0.95)$ & $0.59(0.01-0.88)$ \\
\hline
\end{tabular}

Table 6. Reproducibility of BOLD MRI in the calf.

Values are presented as mean $\pm \mathrm{SD} ; \mathrm{rT} 2_{\max }$, relative change in T2*; TTP, time-to-peak; ap $=0.97 ;$ bp $<0.01$. 


\section{Discussion}

The reproducibility of morphological and functional MRI techniques able to assess the vascular status in patients with PAD and healthy volunteers was evaluated. At morphological level we presented an easy and reproducible method to quantify the number of arteries with a detection limit of $0.5 \mathrm{~mm}$ and a method to assess the diameter of the collateral re-entrance zone in the upper leg. For vascular function we investigated the reproducibility of three different MRI techniques to assess macrovascular popliteal artery blood flow and microvascular calf muscle perfusion and blood oxygenation. Measurement of peak arterial flow in the PA proved reproducible, whereas all DCE MRI-derived parameters and the $r T 2_{\max }$ parameter for BOLD MRI in the lower leg are poorly reproducible in PAD patients. TTP in BOLD MRI was identified as a fairly reproducible microvascular parameter.

Objective assessment of the hemodynamic consequences of stenoses and occlusions in the peripheral arterial tree remains an area of high interest in the diagnostic workup and treatment of patients with PAD. A simple and cost-effective way to assess the functional severity of PAD is the ankle-brachial index (ABI), which is the ratio of the systolic blood pressures measured at the level of the ankle and the upper arm (32). However, the $A B I$ is a relatively crude parameter as it is not able to pinpoint the exact location of a stenosis, is unreliable in patients with an uncompressible vessel wall due to arterial calcifications, and shows a large interobserver variability (33).

Other known techniques to determine vascular function are Doppler based flow measurements, plethysmography, skin perfusion pressure measurements, positron emission tomography (PET), contrastenhanced ultrasound (CEUS) and intra-arterial pressure and/or flow measurements (34-43). Unfortunately, these methods either lack the spatial resolution or coverage to directly measure the effect of vascular adaptations (e.g. collateral vessel formation or changes in microcirculation), or are unfavorable for repeated measurements for instance in the context of therapy monitoring due to invasiveness, use of ionizing 
radiation, or large interobserver variability (33). MRI has the potential to overcome these limitations and can facilitate a variety of functional measurements at both the macro- and microvascular level. In particular, MRI may enable comprehensive diagnostic imaging of both the anatomy of the peripheral vascular tree as well as vascular function in terms of macrovascular flow and surrogate parameters of tissue perfusion and oxygenation $(9,10,13,21,25,44,45)$. MRI-based functional measurements have the advantage that they can be applied repeatedly without detrimental side effects and are easy to add to clinical routine CE-MRA examinations.

We sought to investigate whether MRI can be used to combine its well known high-quality depiction of peripheral vascular anatomy with non-invasive assessment of macrovascular and microvascular hemodynamics (12). Combined assessment of vascular morphology and function using one non-invasive accurate MRI exam is of high clinical interest for the diagnostic workup as well as for monitoring the effects of novel therapeutic strategies in patients with PAD. Although all of the MRI techniques as applied in this study have been described previously (9$11,13)$, little is known about the reproducibility of flow, perfusion and oxygenation sensitive MRI in the lower extremity. To our knowledge, this is the first study on interscan and interreader reproducibility of quantitative MRI derived morphological and functional measures in the context of PAD.

\section{Vascular morphology}

Artery count. Interscan reproducibility for the artery count, as expressed by various measures of agreement and reliability, was high in both patients and healthy volunteers. The RC indicates the smallest noticeable increase in the number of visualized arteries that can be confidently determined by the current combination of image acquisition and analysis methods. Lower RC values signify the capability to detect smaller differences in (longitudinal) follow-up studies. In both patients and volunteers the interscan RC was approximately 1 artery per plane, meaning that a mean increase of one or more artery per plane obtained 


\section{CHAPTER 2}

at two different time-points can be detected beyond measurement error. In other words, any additionally formed or sufficiently matured artery, exceeding a diameter of $0.5 \mathrm{~mm}$, will be detectable during treatment monitoring in PAD patients, using the sequence parameters as described above.

The RC at interreader level was roughly four times as high as the RC at interscan level. This indicates that when different readers are used and an increase of less than four collateral arteries is detected, it cannot be concluded with certainty that this increase represents a real change. This larger RC at interreader level is probably the result of differences in experience of the MRI readers and in reading conditions. Given the larger RC at interreader level, it would be advisable to use the same observer at different points in time. This is not a surprising finding as advanced post-processing techniques are often dependent on the experience of the observer performing the measurements, as has also been demonstrated for abdominal aortic neck volumetry and diameter assessment, which shows good intra-observer, but poor interobserver reliability (46).

The interreader ICC was evidently lower in healthy volunteers compared to patients. A low ICC is unfavorable, as it compromises reproducibility. However, the lower ICC for healthy volunteers can be explained by the fact that these volunteers represent a more homogenous group of subjects, with lower $\mathrm{SD}_{\mathrm{bs}}$ (table 2 ), as opposed to patients harboring a wider range of vascular pathology. Because of this lower $\mathrm{SD}_{\mathrm{bs}}$ and comparable $\mathrm{SD}_{\mathrm{ws}}$ for patients and volunteers, the $\mathrm{SD}_{\mathrm{ws}}$ has more influence on the ICC and the proportion of total variance that is explained by $S D_{b s}$ is smaller. A significantly higher number of arteries per plane was found in patients with PAD compared to healthy volunteers. This was to be expected, as patients were selected for this study based on the presence of collateral arteries. Volunteers did not suffer from PAD and did not have an extensive collateral network. Instead, only the terminal muscular branches of the deep femoral artery were counted. In this study we therefore studied the two extremes of the possible spectrum of disease that may be encountered in clinical practice: a patho- 
logic vascular system with diseased conduit arteries, muscular branches and extensive collateralization versus a healthy vascular tree with patent conduit arteries and muscular branches but lacking collateral arteries. For determination of reproducibility of artery count, which was the primary aim of this study, these differences appeared to be of only minimal influence, indicating the robustness of this method.

Artery diameter. Interscan reproducibility was reasonable for diameter assessment in patients and volunteers, with an average RC of approximately $1 \mathrm{~mm}$. A better RC would not have been realistic given the voxel sizes used in the imaging protocol. Interreader reproducibility in patients was less favorable than interscan reproducibility, as was the case for the artery count. Therefore, in patients, for follow-up studies the same image reader for one patient is advised. Nevertheless, in absolute terms the differences between interscan and interreader reproducibility were very small. In patients the RC at both interscan and interreader level differed only $0.7 \mathrm{~mm}$, which is actually lower than the resolution of the images. In volunteers, interreader reproducibility was equal to or slightly better than interscan reproducibility. This can be explained by the fact that the physiological variance in volunteers with healthy arteries (which influences interscan reproducibility) is somewhat higher than interreader variance.

Given the good reproducibility and especially the low RC values, diameter assessment of the collateral re-entrance zone may help to study morphologic changes due to collateral formation, as we expect an increase in diameter over time of this re-entrance zone when collateral arteries are developing and blood supply distal to a high-grade stenosis or occlusion improves.

In this study we did not find a significant difference in artery diameter between patients and volunteers, although a smaller diameter in patients might have been expected. This finding may be explained by a difference in location at which the diameter was assessed between subjects. In volunteers the measurement was always performed $10 \mathrm{~cm}$ above the distal margin of the femoral condyls, whereas in patients the assessment was performed $5 \mathrm{~cm}$ distal to the vascular lesion (in most 


\section{ChAPTER 2}

cases the popliteal artery), resulting in a large variance. More importantly, however, is the fact that we only included patients with already welldeveloped collateral arteries, that reconstituted the main conduit artery distal to the occlusion to a near-normal diameter.

Image quality. All examinations were of sufficient quality for analysis, with the exception of one subtracted image stack in a PAD patient. A lower CNR in patients is most likely inherent to the presence of PAD, as impaired flow due to vascular lesions will result in lower signal intensity in the vessels distal to vascular lesions. In addition, for patients it is often harder to lie perfectly still during the examination, which results in increased noise levels due to subtraction misregistration.

Source versus subtracted images. An important insight from this study is the finding that image subtraction leads to a deterioration of the reproducibility of the artery count compared to the detection of small arteries on source images. This is surprising because image subtraction leads to better background suppression and better visualization of small vascular structures (47), and is therefore widely applied in clinical practice. A possible explanation for this finding is that small subtraction misregistration errors as well as the well-known $\sqrt{ } 2$ increase in image noise after subtraction apparently do not disturb qualitative evaluation of peripheral MR angiograms for purposes of stenosis detection, but do influence quantitative assessment of the number of arteries as well as arterial diameter.

\section{Vascular function}

PCA MRI. We found favorable values for the interscan and interreader reproducibility measures for the peak arterial flow in the popliteal artery in both patients and healthy volunteers, indicating good reproducibility of PCA MRI over a broad range of flow conditions. Although the interscan $\mathrm{CV}$ in patients run up to $15.8 \%$, both CV and RC were clearly lower than the relative and absolute differences in peak flow between PAD patients and healthy volunteers, making the PCA derived peak flow perfectly suitable for a reliable, objective gauge of PAD severity and therapy monitoring. Minor variations in interscan results are 
probably the result of biologic variation in popliteal artery flow over time and small differences in scan geometry between the two examinations in the subjects.

$D C E M R I$. In contrast to PCA MRI, the interscan reproducibility of DCE MRI was poor in patients, with a CV and RC evidently larger than the relative and absolute differences between patients and healthy volunteers. This implies that repeated measurements over time cannot provide reliable information on small changes in DCE MRI derived calf muscle perfusion using the imaging protocol described in this study. Reproducibility studies in skeletal muscle are unfortunately scarce. Previous DCE MRI studies in various tumor types and skeletal muscle performed under resting conditions also reported unfavorable reproducibility measures, particularly for muscle tissue $(9,21)$. In agreement with our study, the AUC parameter appeared to be more reproducible than the corresponding $K_{i}$ (proportional to $K^{\text {trans }}$ in Ref (19)). In the study by Galbraith et al the flow level of resting state muscle was low and likely prone to large day-to-day variations in the endothelial function (i.e. endothelial permeability and bioavailability of nitric oxide) $(21,48)$. Indeed, T1 values, as determined during the pre-contrast T1 mapping, showed high interscan reproducibility in both patients (mean T1 $578 \pm$ $148 \mathrm{~ms}, \mathrm{CV} 2.7 \%$, RC $43 \mathrm{~ms}$ and ICC 0.99) and healthy volunteers (mean T1 $480 \pm 28 \mathrm{~ms}, \mathrm{CV} 3.1 \%$, RC $42 \mathrm{~ms}$ and ICC 0.71 ), indicating that the technique itself was reproducible. However, the physiologic process we studied was not. To obtain a higher flow level, which better represented physical activity, we used a cuff inflation-deflation protocol to measure muscle perfusion under reactive hyperemia conditions. Application of such a cuff-compression technique is likely to be more reproducible than exercise paradigms, because this paradigm is independent of the patient's compliance, can be standardized, is less hampered by motion artifacts of the leg, and induces a general reactive hyperemia in all calf muscles in contrast to exercise paradigms which activate specific calf muscles (13). Despite these advantages, the interscan reproducibility revealed large day-to-day variations. 


\section{ChAPTER 2}

Interreader reproducibility proved high, indicating a low reader dependency of the measurement, which is desirable for application in therapy monitoring and follow-up studies.

$B O L D$ MRI. Although better than the interscan reproducibility of DCE MRI, the interscan reproducibility for $\mathrm{rT}_{\text {max }}$ in BOLD MRI was also found to be poor. TTP, however, was fairly reproducible in both patients and healthy volunteers. Analogous to DCE MRI, the BOLD technique showed good interscan reproducibility, as proven by the interscan reproducibility of $\mathrm{T2}^{*}$ relaxation times, prior to the actual BOLD MRI (mean T2* $24.5 \pm 1.2 \mathrm{~ms}, \mathrm{CV} 3.7 \%$, RC $2.5 \mathrm{~ms}$ and ICC 0.47 in patients and $23.1 \pm 3.5 \mathrm{~ms}, \mathrm{CV} 12.3 \%$, RC $8 \mathrm{~ms}$ and ICC 0.31 in healthy volunteers respectively), indicating large biologic day-to-day variation in muscle tissue oxygenation, resulting in poor interscan reproducibility for $\mathrm{rT}_{2 \max }$ in the dynamic BOLD measurement. This means that small changes in muscle tissue oxygenation, as reflected by changes in $\mathrm{rT}_{\max }$ cannot be measured reliably with BOLD MRI.

Macrovascular versus microvascular reproducibility. In this study we found that macrovascular flow can be reliably determined by PCA MRI, whereas methods used to measure microvascular function were subject to large interscan variations, with the exception of the TTP in BOLD MRI. These results corroborate to the findings of $\mathrm{Wu}$ et al showing variations in the perfusion of the soleus muscle of healthy volunteers of up to $20 \%$ within one hour using a similar cuff compression method and arterial spin labeling at 3 Tesla (34).

Our observation of poor microvascular interscan reproducibility is remarkable given the reproducible PA flow, and thus blood supply to calf muscle. There might be two underlying reasons for this apparent discrepancy. First, the microcirculation of the calf muscle itself is likely more prone to day-to-day variations than macrovascular flow as the microcirculation and endothelial function are influenced by many intrinsic (i.e. hormonal and circadian cycles, insulin supply, shunting and rerouting of blood) and extrinsic (i.e. outside temperature, season, time of the day, food, pharmacologic) factors (49). Many of these factors are susceptible to day-to-day variations and will therefore inevitably nega- 
tively impact reproducibility of microvascular function. A second factor that might partly explain our findings is that microvascular function was assessed under reactive hyperemic conditions, where as the PA flow was measured under resting conditions.

The better interscan reproducibility of BOLD MRI over DCE MRI might be explained by the fact that DCE MRI parameters ( $K_{i}$ and AUC) are determined by processes that occur within a very short period of time (less than 20 seconds) and depend on endothelial function to a large extent (i.e. extravasation of contrast agent), whereas TTP for BOLD MRI is a much slower process (taking up to two minutes) not depending on endothelial leakage, indicating that the endothelial function has less influence on the results.

\section{Clinical perspective}

Reproducible non-invasive imaging tools are needed for studying the processes of vascular adaptations and collateral development in patients with PAD. Our method can be used to visualize and reproducibly quantify collateral vessel formation over time for monitoring vascular adaptive responses to various treatments in patients with PAD. As interscan reproducibility was evidently better compared to the interreader reproducibility for both artery count and diameter assessment in most cases, it would be advisable to use the same image reader in follow-up studies. Under these conditions, the sensitivity of CE-MRA for the detection of new collateral arteries is very high and allows the detection of an increase of more than one vessel per plane or an increase of more than $1 \mathrm{~mm}$ in diameter of the collateral re-entrance zone beyond measurement error.

At functional level, we demonstrated that PCA MRI can reproducibly determine peak blood flow in the popliteal artery and can reliably detect changes in macrovascular blood flow over time. Although our data are not directly comparable to those of previous studies, who mainly focused on mean flow $(16,18)$, we confirmed the findings of Mohajer et al, by detecting a decrease in blood flow distal to vascular lesions in the superficial femoral artery in patients (10). The high in- 


\section{CHAPTER 2}

terreader reproducibility, indicating a low observer dependency of the measurements, and short acquisition duration, allow easy implementation of flow measurements combined with routine CE-MRA examinations in clinical practice. Further studies are needed to determine the potential of this technique to discriminate between normal and clinically relevant low flow levels in relation to functional parameters such as walking distance and quality of life.

Taken together, the results of the DCE and BOLD MRI indicate that the perfusion responses in the calf muscles of patients with PAD were slower compared to healthy volunteers. However, detecting perfusion differences between patients and healthy volunteers was not the primary goal of this study. Such observable differences will strongly depend on the type of patients and controls included. Anyhow, this finding demonstrates the potential of these MRI techniques to distinguish between different perfusion levels and supports the findings of previous studies comparing patients and controls. Despite the discriminative ability of microvascular functional MRI, DCE MRI does not appear to be a reliable method to quantify changes in the functional status of the microcirculation over time. Additional studies are required to investigate whether better standardization of patient conditions and/or protocols will improve the reproducibility of functional MRI techniques and to determine the clinical applicability of microvascular MRI to assess skeletal muscle perfusion.

\section{Study limitations}

The presented method for the artery count allows quantification of the total number of arteries in the upper leg, although it cannot differentiate between pre-existing large conduit arteries, muscular branches and collateral arteries. This method may well be used for follow-up studies evaluating the effectiveness of therapies that stimulate collateral vessel formation, since even a very small increase in the number of collateral arteries, with a caliber exceeding $0.5 \mathrm{~mm}$, will be detectable. Furthermore, review of the source images allows easy identification of the large named arteries in the upper leg. 
A comparison between conventional digital subtraction angiography (DSA) and CE-MRA to quantify the number of collaterals would have been useful. Unfortunately for most patients no DSA images were available. Previous results of our research group in animal models show that DSA consistently identifies more and smaller collateral arteries (50). However, in the study by De Lussanet it is argued that these small collateral arteries do not add substantially to the blood supply (50).

In clinical practice, MRA examinations of PAD patients are used to visualize the entire peripheral arterial tree from the infrarenal aorta down to the feet. Although most collaterals of the upper leg are located near the adductor canal as previously found by Wecksell et al (51), we imaged the entire volume of the thigh for maximal axial coverage. This resulted in increased acquisition duration of the upper leg station (on average $40-50$ seconds instead of the usual $10-15$ seconds). Also, we did not assess reproducibility of the measurement in the pelvic and lower leg stations in this study. A future improvement could be the use of a blood pool contrast agent as this would allow a first-pass CE-MRA of the upper leg using our extended field of view for artery quantification, followed by high-resolution steady-state images of the total peripheral arterial tree for clinical assessment of the peripheral vasculature $(52,53)$. A disadvantage of this approach is the venous enhancement during the steady state, however, due to the high resolution easy differentiation between arteries and veins is possible $(52,53)$.

For analysis of DCE MRI data we used a standardized vascular input function (VIF). Although an individual VIF could potentially be more accurate, we were not able to identify suitable arteries in the majority of the patients ( 5 out of 8 ) and in 2 healthy volunteers. Low spatial resolution most likely explains the failure to identify a suitable artery in these subjects. Although increased spatial resolution might improve the determination of an individual VIF, it would directly worsen the signalto-noise ratio and the temporal sampling conditions of the rapidly increasing MRI signal. Furthermore, reproducibility measures were even worse in selected cases where an individual VIF could be determined (data not shown). 


\section{CHAPTER 2}

\section{Conclusion}

In conclusion, quantification of the morphological vascular status by CE-MRA, as well as PCA MRI to assess macrovascular blood flow proved highly reproducible in both PAD patients and healthy volunteers and might therefore be helpful in studying the development of collateral arteries in PAD patients and in unraveling the mechanisms underlying this process.

Functional assessment of the microvascular status using DCE and BOLD MRI did not prove reproducible and is therefore currently not suitable for clinical monitoring in PAD. 


\section{References}

1. Norgren L, Hiatt WR, Dormandy JA, et al. Inter-Society Consensus for the Management of Peripheral Arterial Disease (TASC II). J Vasc Surg. 2007;45 Suppl S:S5-67.

2. Leng GC, Fowler B, Ernst E. Exercise for intermittent claudication. Cochrane Database Syst Rev. 2000(2):CD000990.

3. Stewart KJ, Hiatt WR, Regensteiner JG, et al. Exercise training for claudication. N Engl J Med. 2002;347(24):1941-51.

4. Markkanen JE, Rissanen TT, Kivela A, et al. Growth factor-induced therapeutic angiogenesis and arteriogenesis in the heart--gene therapy. Cardiovasc Res. 2005;65(3):656-64.

5. Tammela T, Enholm B, Alitalo K, et al. The biology of vascular endothelial growth factors. Cardiovasc Res. 2005;65(3):550-63.

6. Aghi M, Chiocca EA. Contribution of bone marrow-derived cells to blood vessels in ischemic tissues and tumors. Mol Ther. 2005;12(6):994-1005.

7. Wahlberg E. Angiogenesis and arteriogenesis in limb ischemia. J Vasc Surg. 2003;38(1):198-203.

8. Hiatt WR, Regensteiner JG, Hargarten ME, et al. Benefit of exercise conditioning for patients with peripheral arterial disease. Circulation. 1990;81(2):602-9.

9. Thompson RB, Aviles RJ, Faranesh AZ, et al. Measurement of skeletal muscle perfusion during postischemic reactive hyperemia using contrastenhanced MRI with a step-input function. Magn Reson Med. 2005;54(2):289-98.

10. Mohajer K, Zhang H, Gurell D, et al. Superficial femoral artery occlusive disease severity correlates with MR cine phase-contrast flow measurements. J Magn Reson Imaging. 2006;23(3):355-60.

11. de Vries M, Nijenhuis RJ, Hoogeveen RM, et al. Contrast-enhanced peripheral MR angiography using SENSE in multiple stations: feasibility study. J Magn Reson Imaging. 2005;21(1):37-45.

12. Leiner $\mathrm{T}$, Kessels AG, Nelemans PJ, et al. Peripheral arterial disease: comparison of color duplex US and contrast-enhanced MR angiography for diagnosis. Radiology. 2005;235(2):699-708. 


\section{CHAPTER 2}

13. Ledermann HP, Schulte AC, Heidecker HG, et al. Blood oxygenation leveldependent magnetic resonance imaging of the skeletal muscle in patients with peripheral arterial occlusive disease. Circulation. 2006;113(25):2929-35.

14. de Vries M, de Koning PJ, de Haan MW, et al. Accuracy of semiautomated analysis of 3D contrast-enhanced magnetic resonance angiography for detection and quantification of aortoiliac stenoses. Invest Radiol. 2005;40(8):495-503.

15. Voth M, Haneder S, Huck K, et al. Peripheral magnetic resonance angiography with continuous table movement in combination with high spatial and temporal resolution time-resolved MRA With a total single dose $(0.1$ $\mathrm{mmol} / \mathrm{kg}$ ) of gadobutrol at 3.0 T. Invest Radiol. 2009;44(9):627-33.

16. Meyer RA, Foley JM, Harkema SJ, et al. Magnetic resonance measurement of blood flow in peripheral vessels after acute exercise. Magn Reson Imaging. 1993;11(8):1085-92.

17. Bakker CJ, Kouwenhoven M, Hartkamp MJ, et al. Accuracy and precision of time-averaged flow as measured by nontriggered 2D phase-contrast MR angiography, a phantom evaluation. Magn Reson Imaging. 1995;13(7):959-65.

18. Klein WM, Bartels LW, Bax L, et al. Magnetic resonance imaging measurement of blood volume flow in peripheral arteries in healthy subjects. J Vasc Surg. 2003;38(5):1060-6.

19. Prakash A, Garg R, Marcus EN, et al. Faster flow quantification using sensitivity encoding for velocity-encoded cine magnetic resonance imaging: in vitro and in vivo validation. J Magn Reson Imaging. 2006;24(3):676-82.

20. Isbell DC, Epstein FH, Zhong $X$, et al. Calf muscle perfusion at peak exercise in peripheral arterial disease: measurement by first-pass contrast-enhanced magnetic resonance imaging. J Magn Reson Imaging. 2007;25(5):1013-20.

21. Galbraith SM, Lodge MA, Taylor NJ, et al. Reproducibility of dynamic contrast-enhanced MRI in human muscle and tumours: comparison of quantitative and semi-quantitative analysis. NMR Biomed. 2002;15(2):132-42.

22. Barrett $T$, Brechbiel M, Bernardo $M$, et al. MRI of tumor angiogenesis. J Magn Reson Imaging. 2007;26(2):235-49.

23. Noseworthy MD, Bulte DP, Alfonsi J. BOLD magnetic resonance imaging of skeletal muscle. Semin Musculoskelet Radiol. 2003;7(4):307-15. 
24. Duteil S, Wary C, Raynaud JS, et al. Influence of vascular filling and perfusion on BOLD contrast during reactive hyperemia in human skeletal muscle. Magn Reson Med. 2006;55(2):450-4.

25. Baudelet C, Cron GO, Gallez B. Determination of the maturity and functionality of tumor vasculature by MRI: correlation between BOLD-MRI and DCE-MRI using P792 in experimental fibrosarcoma tumors. Magn Reson Med. 2006;56(5):1041-9.

26. Stainsby JA, Wright GA. Monitoring blood oxygen state in muscle microcirculation with transverse relaxation. Magn Reson Med. 2001;45(4):66272.

27. Bulte DP, Alfonsi J, Bells S, et al. Vasomodulation of skeletal muscle BOLD signal. J Magn Reson Imaging. 2006;24(4):886-90.

28. Haacke M, Brown R, Thompson M, et al. T1 estimation from SSI measurements at multiple flip angles. In: Haacke M, Brown R, Thompson M, et al., eds. Magnetic resonance imaging: physical principles ans sequence design. New York, NY: John Willey \& Sons, Inc; 1999:654-661.

29. Bland JM, Altman DG. Measurement error. BMJ. 1996;312(7047):1654.

30. Jansen JF, Kooi ME, Kessels AG, et al. Reproducibility of quantitative cerebral T2 relaxometry, diffusion tensor imaging, and $1 \mathrm{H}$ magnetic resonance spectroscopy at 3.0 Tesla. Invest Radiol. 2007;42(6):327-37.

31. Bland JM, Altman DG. Statistical methods for assessing agreement between two methods of clinical measurement. Lancet. 1986;1(8476):30710.

32. Begelman SM, Jaff MR. Noninvasive diagnostic strategies for peripheral arterial disease. Cleve Clin J Med. 2006;73 Suppl 4:S22-9.

33. van Langen $H$, van Gurp J, Rubbens L. Interobserver variability of anklebrachial index measurements at rest and post exercise in patients with intermittent claudication. Vasc Med. 2009;14(3):221-6.

34. Wu WC, Wang J, Detre JA, et al. Hyperemic flow heterogeneity within the calf, foot, and forearm measured with continuous arterial spin labeling MRI. Am J Physiol Heart Circ Physiol. 2008;294(5):H2129-36.

35. Burchert W, Schellong S, van den Hoff J, et al. Oxygen-15-water PET assessment of muscular blood flow in peripheral vascular disease. J Nucl Med. 1997;38(1):93-8.

36. Bragadeesh T, Sari I, Pascotto M, et al. Detection of peripheral vascular stenosis by assessing skeletal muscle flow reserve. J Am Coll Cardiol. 2005;45(5):780-5.

37. Peetrons P. Ultrasound of muscles. Eur Radiol. 2002;12(1):35-43. 


\section{CHAPTER 2}

38. Chloros GD, Smerlis NN, Li Z, et al. Noninvasive evaluation of upperextremity vascular perfusion. J Hand Surg Am. 2008;33(4):591-600.

39. Weber MA, Krix M, Delorme S. Quantitative evaluation of muscle perfusion with CEUS and with MR. Eur Radiol. 2007;17(10):2663-74.

40. Ludman PF, Volterrani M, Clark AL, et al. Skeletal muscle blood flow in heart failure measured by ultrafast computed tomography: validation by comparison with plethysmography. Cardiovasc Res. 1993;27(6):1109-15.

41. Duet M, Virally M, Bailliart O, et al. Whole-body (201)TI scintigraphy can detect exercise lower limb perfusion abnormalities in asymptomatic diabetic patients with normal Doppler pressure indices. Nucl Med Commun. 2001;22(9):949-54.

42. Ament W, Lubbers J, Rakhorst G, et al. Skeletal muscle perfusion measured by positron emission tomography during exercise. Pflugers Arch. 1998;436(5):653-8.

43. Fleischer AC, Niermann KJ, Donnelly EF, et al. Sonographic depiction of microvessel perfusion: principles and potential. J Ultrasound Med. 2004;23(11):1499-506.

44. Carlier PG, Bertoldi D, Baligand C, et al. Muscle blood flow and oxygenation measured by NMR imaging and spectroscopy. NMR Biomed. 2006;19(7):954-67.

45. Lebon V, Brillault-Salvat C, Bloch G, et al. Evidence of muscle BOLD effect revealed by simultaneous interleaved gradient-echo NMRI and myoglobin NMRS during leg ischemia. Magn Reson Med. 1998;40(4):551-8.

46. Diehm N, Kickuth $\mathrm{R}$, Gahl B, et al. Intraobserver and interobserver variability of 64-row computed tomography abdominal aortic aneurysm neck measurements. J Vasc Surg. 2007;45(2):263-8.

47. Sueyoshi E, Sakamoto I, Matsuoka Y, et al. Symptomatic peripheral vascular tree stenosis. Comparison of subtracted and nonsubtracted 3D contrast-enhanced MR angiography with fat suppression. Acta Radiol. 2000;41(2):133-8.

48. Ziegler MA, Distasi MR, Bills RG, et al. Marvels, mysteries, and misconceptions of vascular compensation to peripheral artery occlusion. Microcirculation. 2010;17(1):3-20.

49. Deanfield J, Donald A, Ferri C, et al. Endothelial function and dysfunction. Part I: Methodological issues for assessment in the different vascular beds: a statement by the Working Group on Endothelin and Endothelial Factors of the European Society of Hypertension. J Hypertens. 2005;23(1):7-17. 
50. de Lussanet QG, van Golde JC, Beets-Tan RG, et al. Magnetic resonance angiography of collateral vessel growth in a rabbit femoral artery ligation model. NMR Biomed. 2006;19(1):77-83.

51. Wecksell MB, Winchester PA, Bush HL, Jr., et al. Cross-sectional pattern of collateral vessels in patients with superficial femoral artery occlusion. Invest Radiol. 2001;36(7):422-9.

52. Hadizadeh DR, Gieseke J, Lohmaier SH, et al. Peripheral MR angiography with blood pool contrast agent: prospective intraindividual comparative study of high-spatial-resolution steady-state MR angiography versus standard-resolution first-pass MR angiography and DSA. Radiology. 2008;249(2):701-11.

53. Wang MS, Haynor DR, Wilson GJ, et al. Maximizing contrast-to-noise ratio in ultra-high resolution peripheral MR angiography using a blood pool agent and parallel imaging. J Magn Reson Imaging. 2007;26(3):580-8. 



\section{CHAPTER 3}

MRI derived arterial peak flow in peripheral

arterial disease: Towards a standardized

measurement

B. Versluis, T. Leiner, P.J. Nelemans, R. Brans, J.E. Wildberger, G.W.H. Schurink, W.H. Backes

Accepted for publication in the European Journal of Vascular and Endovascular Surgery 


\section{CHAPTER 3}

\section{Abstract}

\section{Objectives}

In patients with peripheral arterial disease, it would be clinically desirable to have a standardized arterial position to measure the arterial peak flow (APF) independent of the location of obstructive arterial lesions with well-known reference values and high ability to differentiate between diseased and healthy arterial vasculature. The purpose of this study was to determine the best location to measure the APF in order to facilitate clinical standardization.

\section{Materials and Methods}

259 patients with varying degrees of peripheral artery disease (PAD) and 48 patients without PAD were included. All subjects underwent vector cardiography-synchronized quantitative 2D cine MR phasecontrast velocity imaging at three predefined arterial positions (i.e. common femoral artery (CFA), superficial femoral artery (SFA) and popliteal artery (PA)). APF values of PAD patients with clinically relevant stenosis according to MRA were compared with non-PAD patients. Discriminative ability to identify PAD was evaluated by means of receiver-operatoring characteristic (ROC) curves and the corresponding areas under the curve (AUC). Furthermore, failure and success rates of APF measurements at all arterial positions were evaluated.

\section{Results}

Mean APF values in PAD patients were reduced by $42 \%, 55 \%$ and $59 \%$ compared to non-PAD patients for respectively the CFA, SFA and PA $(p<0.01)$. The AUC's were $0.84,0.92$ and 0.93 for CFA, SFA and PA, respectively. Success rates of the measurements in both PAD and nonPAD patients were $90 \%, 91 \%$ and $93 \%$ for CFA, SFA and PA, respectively. 


\section{Conclusions}

To conclude, the APF measured in the PA and SFA show large differences between PAD and non-PAD patients, strong discriminative ability and high success rates as compared to measurements in the CFS. For the location distal to the majority of arterial lesions, the preferred position for a standardized APF measurement in PAD patients is the PA. 


\section{Introduction}

MR angiography (MRA) has established itself as a highly accurate non-invasive diagnostic imaging method for the morphological evaluation of the peripheral conduit arteries in patients with peripheral arterial disease (PAD) (1-4). Given the progressive character of PAD, an increasing number of patients nowadays undergoes multiple MRA examinations during lifetime for treatment planning and/or therapy monitoring. For those patients, a fast and simple functional MRI derived measure, such as the arterial peak flow (APF), which is suitable to objectively quantify the vascular status and to monitor the progression of PAD over time, might be a valuable addition to $\operatorname{MRA}(5,6)$. Numerous other noninvasive functional diagnostic tools are available for this purpose, such as ankle-brachial pressure index (ABI), duplex ultrasonography and laser Doppler measurements (7-10). APF measurements, however, have the advantage of being a fast, reproducible and highly automated MRI technique, with acquisition times well below one minute, that can easily be added to existing MRA-protocols to obtain morphologic information together with functional vascular status during the same examination (5, 6, 11-13).

Previous studies demonstrated good reproducibility for APF measurements and lower APF values in PAD patients compared to healthy control subjects $(5,6)$. For clinical practice, it would be desirable to have a standardized arterial position to measure the APF, comparable to ABI measurements, independent of the location of obstructive arterial lesions, but with high ability to differentiate between a diseased and healthy arterial vasculature and with well-known reference values. Therefore, in this study the APF was acquired at three distinctive and standardized arterial positions in a large group of PAD and non-PAD patients to determine the location that differentiates best between PAD and non-PAD patients. 


\section{Materials and Methods}

\section{Study population}

MR cine phase-contrast imaging flow measurements are a standard addition to contrast-enhanced MR angiography (CE-MRA) at our institution in all patients scheduled for CE-MRA of the peripheral arteries. The ethics committee waived the need for informed consent for patients, as flow measurements are part of the standard imaging protocol at our institution. Patients with (partial) lower extremity amputations were not included in this study. Otherwise there were no exclusion criteria. Patients referred for CE-MRA of the peripheral arterial tree to rule out deep vein thrombosis or in the pre-operative workup for reconstructive surgery using lower extremity tissue flaps with no clinical symptoms of PAD nor signs of PAD on CE-MRA, were used as reference in this study. Patient characteristics were retrieved from the patients' records and are summarized in table 1.

\begin{tabular}{lcc}
\hline & PAD patients & Non-PAD patients \\
\hline Number & 259 & 48 \\
Males (\%) / Females (\%) & $147(57 \%) / 112(43 \%)$ & $23(48 \%) / 25(52 \%)$ \\
Age (years) & $67 \pm 13.1$ & $53 \pm 16.4 *$ \\
Heart rate (bpm) & $71 \pm 13$ & $75 \pm 14$ \\
Bodyweight (kg) & $77.1 \pm 15.1$ & $75.7 \pm 16.4$ \\
IC/Cl & $137(53 \%) / 122(47 \%)$ & - \\
Diabetics (\%) & $120(54 \%)^{\mathrm{a}}$ & $5(13 \%)^{\mathrm{b}}$ \\
\hline
\end{tabular}

Table 1. Patient characteristics.

Values are presented as mean \pm SD. Bpm, beats per minute; $\mathrm{kg}$, kilogram; IC, intermittent claudication; $\mathrm{Cl}$, critical ischemia." $\mathrm{p}<0.01$

a Diabetic status could only be retrieved in 221 out of 259 PAD patients. bDiabetic status could only be retrieved in 38 out of 48 non-PAD patients. 


\section{MRI protocol}

All patients underwent a three-station CE-MRA protocol of the peripheral arteries (14), followed by quantitative MR cine phase-contrast imaging flow measurements at three distinctive arterial positions as described below. Flow data were used to obtain the APF in PAD and non-PAD patients (15). A schematic overview of the scan protocol is given in figure 1. All examinations were performed using a 1.5-T MRI system (Intera, Philips Medical Systems, Best, The Netherlands). For signal reception we used a dedicated 12-element phased-array peripheral vascular coil with a craniocaudal coverage of $128 \mathrm{~cm}$ (Philips Medical Systems). Patients were imaged in supine position and were in this position for approximately 30 minutes before the flow measurements were started. During this period the CE-MRA examination was performed, using a fixed dose of $10 \mathrm{~mL}$ gadofosveset trisodium (Ablavar ${ }^{\circ}$, Lantheus Medical Imaging, Billerica, MA) as contrast agent.

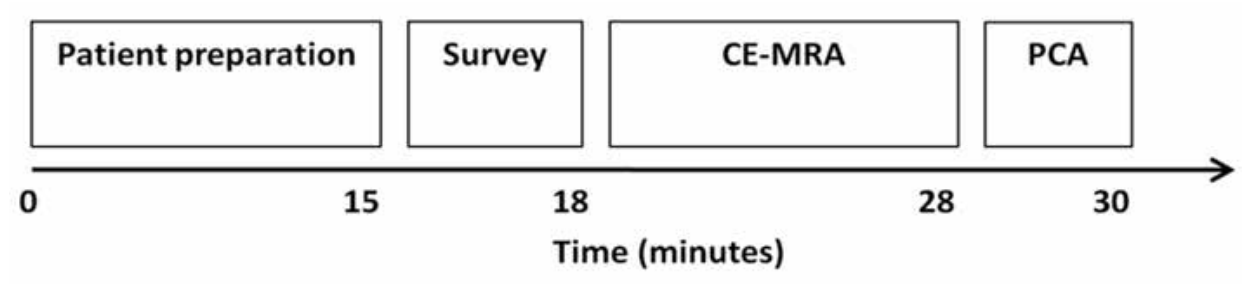

Figure 1. Overview of imaging protocol and the approximate duration of each scan sequence. Contrast-enhanced MR angiography (CE-MRA) was combined with cine phasecontrast imaging ( $\mathrm{PCl}) \mathrm{MRI}$. Total MRI examination, including patient positioning, table movements and MRI scans, took approximately 35 minutes.

Survey. A non-enhanced time-of-flight (TOF) scan of the pelvic, upper and lower leg station was performed to prescribe the imaging volumes of interest for morphological and functional imaging. For this, a turbo field echo pulse sequence was used with a $180^{\circ}$ inversion prepulse to suppress stationary tissues. Thirty-one axial slices per station were acquired with 3.3-mm slice thickness and $11-\mathrm{mm}$ interslice gap, and an inferiorly concatenated saturation band. The standard quadrature body coil was used for signal transmission and reception. For positioning of 
the 3D CE-MRA volumes, maximum intensity projections (MIP) were generated in 3 orthogonal directions.
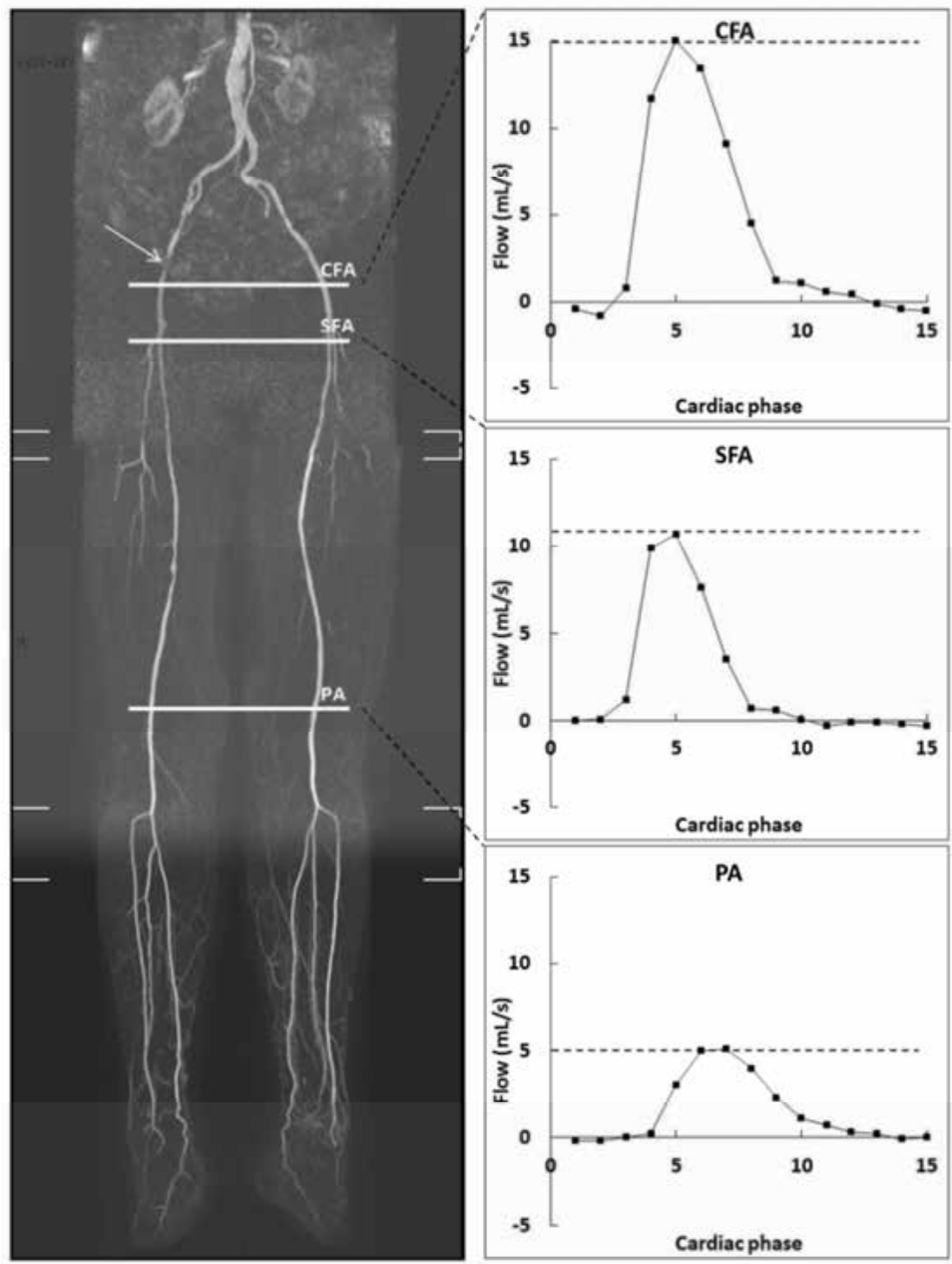

Figure 2. Maximum intensity reconstructions of a CE-MRA acquisitions in a PAD patient with a significant arterial stenosis in the right common femoral artery (CFA), indicated by the arrow. The predefined locations of the arterial peak flow (APF) measurements are indicated by, i.e. the proximal common femoral artery, proximal superficial femoral artery (SFA) and P2-segment of the popliteal artery (PA). The graphics show examples of flow wave forms in this particular patient. The black dotted line in each graphic indicates the level of the APF. 


\section{CHAPTER 3}

CE-MRA. A three-station 3D gradient-echo (fast field echo) MRA sequence was performed as previously described (2, 14). Acquisition parameters were as follows: TR $4.8 \mathrm{~ms}$, TE $1.45 \mathrm{~ms}$, flip angle $40^{\circ}$, FOV $470 \mathrm{~mm}$, matrix 480, and voxel dimensions (reconstructed) $0.92 \times 0.92$ $x 1.20 \mathrm{~mm}$. Prior to contrast agent administration, a non-enhanced 'mask' image data set was acquired with exactly the same acquisition parameters as the CE-MRA, enabling background tissue suppression by image subtraction.

Flow MRI. For quantitative cine phase-contrast imaging we used a 2D gradient-echo (fast field echo) scan technique with the following acquisition parameters: TR $9.7 \mathrm{~ms}$, TE $5.8 \mathrm{~ms}$, flip angle $30^{\circ}$, FOV 380 $\mathrm{mm}$, matrix 384, and reconstructed voxel dimensions of $0.99 \times 0.99 \times$ $6.00 \mathrm{~mm}$. Fifteen dynamic phases were acquired during the cardiac cycle. Measurements were performed at the level of the proximal common femoral artery (CFA), the proximal superficial femoral artery (SFA) and the P2-segment of the popliteal artery (PA) (figure 2). The phase encoding velocity was set to $100 \mathrm{~cm} / \mathrm{s}$ in the craniocaudal direction for CFA and SFA measurements and $60 \mathrm{~cm} / \mathrm{s}$ for PA measurements $(5,15)$. Vector cardiography (VCG) triggering was used for retrospective cardiac synchronization. Parallel imaging (sensitivity encoding, SENSE) was applied to reduce scan time (SENSE acceleration factor 2 in the anteriorposterior direction) (16). At a mean heart rate of 60 beats per minute, the nominal acquisition time was 1 minute. Coronal and sagittal MIP reconstructions of CE-MRA recordings were used for accurate angulation of the slice, perpendicular to the direction of the vessel of interest.

\section{Angiographic reading}

All CE-MRA datasets were analyzed by a well-trained radiologist with over 5 years of experience in MRA of the peripheral vasculature. For this study, CE-MRA data were used to determine the presence and severity of obstructive arterial lesions in PAD and to exclude presence of obstructive arterial lesions or arterial malformations in the group of non-PAD patients. 


\section{Flow analysis}

Modulus and phase images were reconstructed from the cine flow data. A quantitative flow analysis package (QFlow) included with the software release (R11.4.14, Philips Medical Systems) of the MRI hardware was used for analysis of the flow data directly after the acquisition. Using this software, a region of interest (ROI) covering the entire visible cross-section of the artery of interest was accurately drawn manually using a modulus image at peak systole and then automatically propagated to the remaining cardiac phases using an active contour algorithm. Although peak systolic phase only is sufficient to calculate the APF, ROl's were propagated to the remaining cardiac phases to obtain flow wave forms, which were visually analyzed to reassure the chosen cardiac phase was indeed at peak systole and to detect possible aliasing effects. If detected, the measurement was repeated with sufficiently higher phase encoding velocity.

\section{APF analysis}

APF was determined in the most symptomatic leg in patients with $P A D$ and in the right leg in non-PAD patients. If both legs were equally affected in patients with PAD, the right leg APF was used for analysis. Using CE-MRA data, patients with PAD were divided into two subgroups, i.e. patients with and without relevant arterial stenoses in the peripheral arterial tree. A relevant stenosis was defined as $>50 \%$ stenosis in the iliac artery, CFA, SFA or PA, independent of the extensiveness and location of the lesion and/or $>50 \%$ stenosis in at least two out of three main arteries of the lower leg. Stenosis grade of the peripheral arterial tree below $<50 \%$, was considered not relevant. A comparison of APF values was made between PAD patients with and without relevant stenoses in the peripheral arterial tree and non-PAD patients at the level of the CFA, SFA and PA. Also, the ability to discriminate between PAD and non-PAD patients at each level was determined. Furthermore, APF values in PAD patients with arterial lesions limited to the lower leg were compared with non-PAD patients to determine whether APF measure- 


\section{ChAPTER 3}

ments are sensitive for lesions in the out-flow trajectory. Success rates of APF measurements and causes of failure at the distinctive arterial positions were determined and compared.

\section{Statistical analysis}

Statistical analysis was performed with commercially available statistical software (SPSS 16.0, SPSS Inc., Chicago, IL) and STATA (11.2, StataCorp LP., Texas). Mean values between groups were tested for statistical significance using a $t$-test for independent samples. The ability of APF measurements to discriminate between PAD and non-PAD patients was compared using receiver operating characteristic (ROC) curves and areas under the curve (AUC). Differences between the AUCs were tested for statistical significance using the method described by Hanley and McNeil, which accounts for the fact that the AUCs were derived from the same sample of patients (17). In all analysis, p-values $<0.05$ were considered to indicate statistical significance.

\section{Results}

A total of 307 consecutive patients undergoing CE-MRA of the peripheral arteries at our institution as part of a clinical routine examination between January and December 2010 were considered eligible for analysis in this study. Presence of PAD was confirmed by CE-MRA in 259 PAD-patients. Relevant stenoses were present anywhere within the peripheral arterial tree in 238 out of 259 patients with PAD according to CE-MRA, whereas no relevant stenoses were found in 21 patients initially diagnosed with PAD.

In 48 patients CE-MRA was indicated to rule out deep vein thrombosis or acquired in the pre-operative workup of patients scheduled for reconstructive surgery with lower extremity tissue serving as donor site.

APF values in patients with PAD with and without relevant stenoses and non-PAD patients are given in table 2. Mean APF values in PAD patients with relevant stenosis were reduced by $42 \%, 55 \%$ and $59 \%$ 
compared to non-PAD patients for CFA, SFA and PA, respectively ( $p<$ 0.01 at all levels). Differences between PAD patients with and without relevant stenosis were significant at all arterial positions $(p<0.01)$. Differences between PAD patients without relevant stenosis and nonPAD patients were significant as well $(p=0.03, p<0.01$ and $p=0.01$ for CFA, SFA and PA, respectively).

\begin{tabular}{lcccccc}
\hline & \multicolumn{3}{c}{ PAD patients } & \multicolumn{3}{c}{ Non-PAD patients } \\
& \multicolumn{2}{c}{ Relevant stenosis } & No relevant stenosis & \\
& APF $(\mathrm{mL} / \mathrm{s})$ & $\mathbf{n}$ & APF $(\mathrm{mL} / \mathrm{s})$ & $\mathbf{n}$ & APF $(\mathrm{mL} / \mathbf{s})$ & \\
\hline CFA & $17.9 \pm 9.3$ & 212 & $24.9 \pm 9.0$ & 19 & $30.6 \pm 9.1$ & 45 \\
SFA & $9.0 \pm 6.8$ & 211 & $15.8 \pm 5.5$ & 20 & $20.2 \pm 6.4$ & 48 \\
PA & $4.6 \pm 3.0$ & 218 & $8.7 \pm 3.8$ & 21 & $11.3 \pm 3.8$ & 45 \\
\hline
\end{tabular}

Table 2. Arterial peak flow in PAD and non-PAD patients.

Values are presented as mean \pm SD. APF, arterial peak flow; CFA, common femoral artery; SFA, superficial femoral artery; PA, popliteal artery.

In 14 PAD patients arterial lesions were limited to the lower leg (i.e. more than $50 \%$ stenosis in at least two out of the three main arteries of the lower leg). In these patients, APF was $25.4 \pm 9.7 \mathrm{~mL} / \mathrm{s}, 13.6 \pm 4.6$ $\mathrm{mL} / \mathrm{s}$ and $7.6 \pm 4.4 \mathrm{~mL} / \mathrm{s}$ in the CFA, SFA and PA, respectively. The APF in these patients was significantly lower compared to non-PAD patients for the SFA $(33 \%, p<0.01)$ and PA $(33 \%, p<0.01)$, whereas a similar trend was found for the CFA $(17 \%, p=0.07)$.

The distribution of APF values of the (most) symptomatic leg of PAD patients with relevant stenosis and the right leg of non-PAD patients at the level of the CFA, SFA and PA is graphically displayed in figure 3. In general, APF values in PAD patients were lower compared to non-PAD patients, although some overlap exists. Relative differences between PAD and non-PAD patients were larger if the measurement was performed more distally within the peripheral arterial tree. 

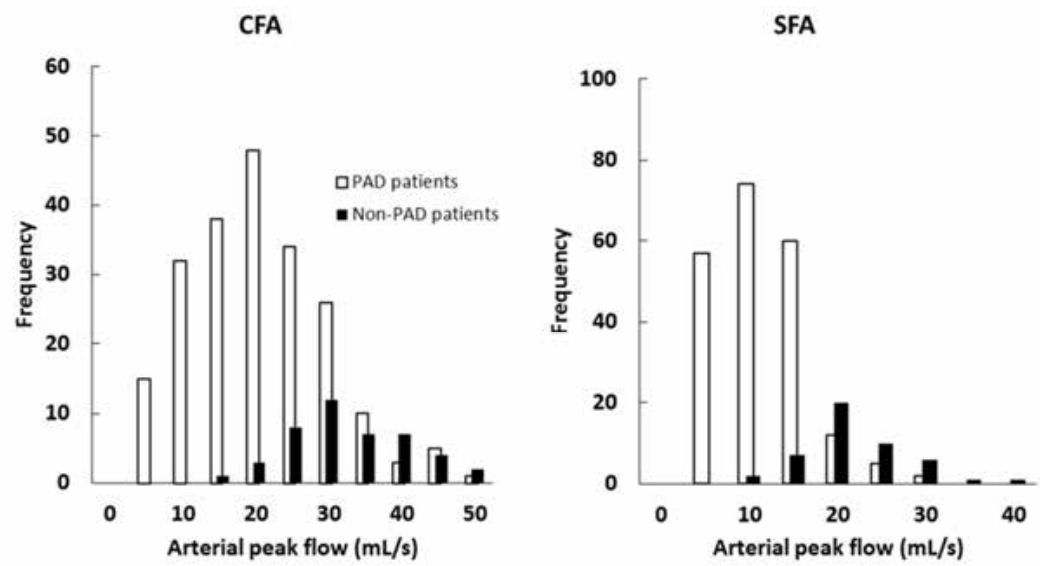

PA

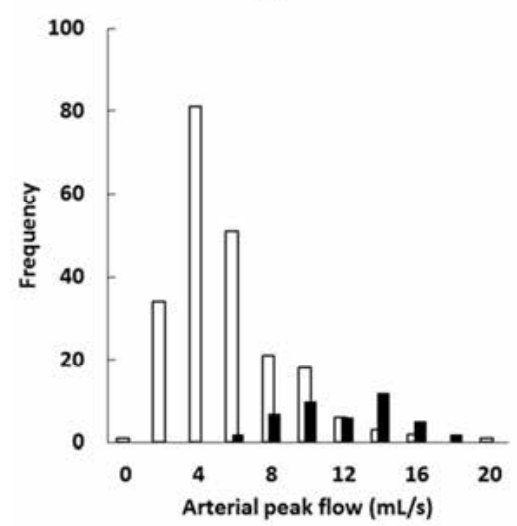

Figure 3. Histograms showing the distribution of arterial peak flow values among a PAD and non-PAD patient population at the level of the common femoral artery (CFA), superficial femoral artery (SFA), and popliteal artery (PA).

The discriminative ability of APF measurements in the CFA, SFA and PA is presented by the ROC curves (figure 4). The area-under-thecurve (AUC) at the level of the CFA was 0.84 with a 95\% confidence interval $(\mathrm{Cl})$ between $0.78-0.89$. At the level of the SFA and PA, AUC's were $0.92(95 \% \mathrm{Cl} 0.88-0.96)$ and $0.92(95 \% \mathrm{Cl} 0.88-0.95)$, respectively. The AUC at the level of the CFA was significantly lower compared to AUC's at the level of the SFA and PA, whereas no statistically significant difference was found between the level of the SFA and PA. 
Causes of failure of APF measurements are given in table 3. Operator errors mainly consisted of wrong localization of the slices of the flow measurements.

\begin{tabular}{lccc}
\hline Arterial position & PAD patients & Non-PAD patients & Total \\
\hline CFA & $28(11 \%)$ & $3(6 \%)$ & $31(10 \%)$ \\
Operator error & 10 & 2 & 12 \\
Occlusion & 14 & 0 & 14 \\
Orthopedic prothesis & 4 & 0 & 4 \\
SFA & $28(12 \%)$ & $0(0 \%)$ & $28(9 \%)$ \\
Operator error & 2 & 0 & 2 \\
Occlusion & 24 & 0 & 24 \\
Orthopedic prothesis & 2 & 0 & 2 \\
PA & $20(8 \%)$ & $3(6 \%)$ & $23(7 \%)$ \\
Operator error & 2 & 2 & 4 \\
Occlusion & 12 & 0 & 12 \\
Orthopedic prothesis & 4 & 1 & 5 \\
Bypass & 2 & 0 & 2 \\
Total & $76(10 \%)$ & $6(4 \%)$ & $82(9 \%)$ \\
\hline
\end{tabular}

Table 3. Main causes of failure for the APF measurements.

APF, arterial peak flow; CFA, common femoral artery; SFA, superficial femoral artery; PA, popliteal artery.

\section{Discussion}

It is clinically desirable to have a standardized arterial position to measure the APF, preferably independent of the location of obstructive arterial lesions, with well-known reference values and high ability to differentiate between diseased and healthy arterial vasculature. The most suitable location for this purpose was expected to be the popliteal artery, as this is the most distally located large conduit artery of the peripheral arterial tree and therefore situated distally from the majority of the obstructive arterial lesions. According to the results of this study, 


\section{CHAPTER 3}

the APF acquired in the popliteal artery indeed shows the largest differences between PAD and non-PAD patients (i.e. 59\% lower APF values in PAD patients), high discriminative ability (AUC of 0.92) and highest success rates of the measurement (up to $92 \%$ in PAD patients).

Objective assessment of the functional consequences of stenoses and occlusions in the peripheral arterial tree is a necessity in diagnosis and longitudinal follow up or therapy monitoring of patients with PAD. Currently, the ankle-brachial index (ABI) measurement is the most recognized and most widely applied functional measurement used for diagnosis and therapy monitoring (4, 18, 19). ABI measurements, however, are hampered by poor reproducibility and cannot be determined accurately in patients with heavily calcified arteries (20-22). Other known techniques to determine vascular function are Doppler based flow measurements, plethysmography, skin perfusion pressure measurements, positron emission tomography (PET), contrast-enhanced ultrasound (CEUS) and intra-arterial pressure and/or flow measurements (23-32). These techniques, however, may lack spatial resolution or coverage to directly measure the effect of vascular adaptations (e.g. collateral vessel formation or changes in microcirculation), or are unfavorable for repeated measurements for instance in the context of therapy monitoring due to invasiveness, use of ionizing radiation, or large interobserver variability (7).

MRA has established itself as a highly accurate non-invasive diagnostic imaging method and is widely applied for diagnosis and treatment planning in PAD (1-4). Due to the progressive character of PAD, an increasing number of patients with PAD undergo multiple MR angiographic examinations during lifetime. For those patients MRI can also be used to obtain objective functional vascular information, for example by measuring arterial peak flow (APF). The APF is defined as the maximum systolic flow and is an attractive arterial flow measure, as the APF is an important determinant of the systolic blood pressure (33), which previously showed relatively large differences between PAD patients and control subjects and proved highly reproducible $(5,6,15)$. 
One of the strengths of other functional tests, for example $A B I$ measurements, is a high degree of standardization, meaning that for example the $A B I$ is measured at the same location with the same technique for every patient. Such a standardized protocol is a necessity for functional measurements to be suitable for longitudinal follow-up or therapy monitoring in patients. As APF measurements are relatively new and not yet widely applied, currently no such standardized protocol does exist, nor are there reference values known. Although APF measurements are relatively fast, it is highly desirable to have a single, predefined location to measure the APF in all patients with PAD, at which the ability to differentiate between diseased and normal vasculature is best.

In this study we demonstrated a standardized approach in which the APF was measured in the proximal CFA, proximal SFA and P2 segment of the PA in patients with PAD, independent from the location of obstructive arterial lesions, and in non-PAD patients. As expected, mean APF values in PAD were significantly lower compared to non-PAD patients at all arterial levels. The largest decrease in APF was found at the level of the PA in patients with PAD with a clinically relevant stenosis, were the average APF was $59 \%$ lower compared to non-PAD patients. A simple explanation for this finding is that flow in PA reflects all proximal obstructive arterial lesions. Interestingly, APF measured in the SFA and PA are both sensitive for proximal disease as well as disease distal to the position of the measurement.

A distinction was made between PAD patients with and without relevant stenoses, as those patients without relevant stenosis were expected to have higher APF values, while therapeutic options in these patients differ from those patients with relevant stenosis. Results of our study confirm this assumption, as APF in PAD patients with relevant stenoses was significantly lower compared to those without stenoses.

Due to the large differences in APF between PAD and non-PAD patients, the discriminative ability of APF measurements as shown by the ROC curves of figure 4 was excellent. The largest AUC was found at the level of the PA (0.93) again, although the difference with the SFA (0.92) 


\section{CHAPTER 3}

was small. The average success rate of APF measurements was high (on average $91 \%$ for all subjects in this study) and best at the level of the PA in both PAD and non-PAD patients.

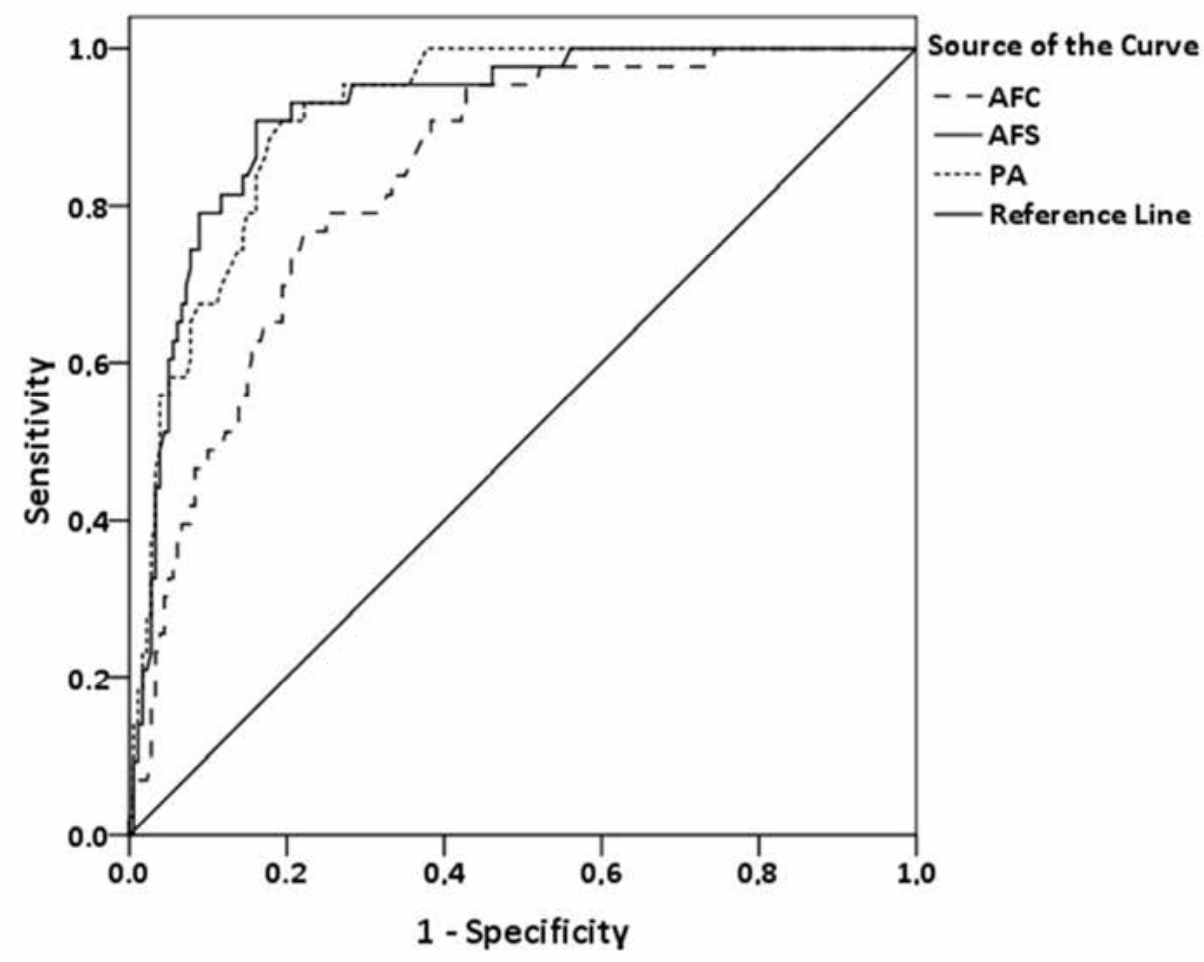

Figure 4. Receiver operating characteristic (ROC) curves among a PAD and non-PAD patient population at the level of the common femoral artery (CFA), superficial femoral artery (SFA), and popliteal artery (PA).

SFA and PA are both excellent positions to measure the APF, independent from the location of obstructive arterial lesions. Nevertheless, we would prefer to measure the APF in the PA as results at this position were only slightly, but consistently, better and the PA is situated distally from the majority of the obstructive arterial lesions.

There are some limitations in this study. Only patients with PAD scheduled for MRA were included. Although this resulted in a selected study population, this population was considered to be clinically the 
most relevant as far as APF measurements are concerned, as MRI-based APF measurements are not (yet) suitable for screening purposes due to the limited availability of MRI systems or magnet time and associated costs. Also, as the selected population comprised consecutive patients undergoing CE-MRA, it is a clinically representative group as far as disease severity is concerned. Nevertheless, this implies that current findings do not necessarily apply for all PAD patients. The group of nonPAD patients who were used as control subjects were free from PAD, according to CE-MRA and anamnesis, but not free from vascular disease, as the majority of the non-PAD patients had deep vein thrombosis. Nevertheless, APF measures of these non-PAD patients were comparable with healthy controls from previous studies $(5,6)$.

Although clinically relevant, APF was not measured at the level of the main arteries of the lower leg as the current spatial resolution would have been insufficient to accurately measure the APF in these small arteries $(13,34,35)$, whereas increasing spatial resolution would have resulted in unsatisfactory acquisition times for clinical practice. Yet, as our results have shown, APF measurements at the level of the SFA and PA proved sensitive for arterial stenosis in the lower leg.

To conclude, the APF measured in the PA and SFA show large differences between PAD and non-PAD patients, strong discriminative ability and high success rates as compared to measurements in the CFS. For the location distal to the majority of arterial lesions, the preferred position for a standardized APF measurement in PAD patients is the PA. 


\section{References}

1. de Vries M, de Koning PJ, de Haan MW, et al. Accuracy of semiautomated analysis of 3D contrast-enhanced magnetic resonance angiography for detection and quantification of aortoiliac stenoses. Invest Radiol. 2005;40(8):495-503.

2. de Vries M, Nijenhuis RJ, Hoogeveen RM, et al. Contrast-enhanced peripheral MR angiography using SENSE in multiple stations: feasibility study. J Magn Reson Imaging. 2005;21(1):37-45.

3. Leiner T, Kessels AG, Nelemans PJ, et al. Peripheral arterial disease: comparison of color duplex US and contrast-enhanced MR angiography for diagnosis. Radiology. 2005;235(2):699-708.

4. Norgren L, Hiatt WR, Dormandy JA, et al. Inter-Society Consensus for the Management of Peripheral Arterial Disease (TASC II). J Vasc Surg. 2007;45 Suppl S:S5-67.

5. Versluis B, Backes WH, van Eupen MG, et al. Magnetic resonance imaging in peripheral arterial disease: reproducibility of the assessment of morphological and functional vascular status. Invest Radiol. 2011;46(1):1124.

6. Versluis B, Dremmen MH, Nelemans PJ, et al. MRI of arterial flow reserve in patients with intermittent claudication: feasibility and initial experience. PLoS One. 2012;7(3):e31514.

7. van Langen $H$, van Gurp J,Rubbens L. Interobserver variability of anklebrachial index measurements at rest and post exercise in patients with intermittent claudication. Vasc Med. 2009;14(3):221-6.

8. Begelman SM, Jaff MR. Noninvasive diagnostic strategies for peripheral arterial disease. Cleve Clin J Med. 2006;73 Suppl 4:S22-9.

9. Corcoran HA, Smith BE, Mathers $\mathrm{P}$, et al. Laser Doppler imaging of reactive hyperemia exposes blood flow deficits in a rat model of experimental limb ischemia. J Cardiovasc Pharmacol. 2009;53(6):446-51.

10. Kido M, Takeuchi S, Hayashida S, et al. Assessment of abnormal blood flow and efficacy of treatment in patients with systemic sclerosis using a newly developed microwireless laser Doppler flowmeter and arm-raising test. Br J Dermatol. 2007;1 57(4):690-7.

11. Dumoulin CL. Phase contrast MR angiography techniques. Magn Reson Imaging Clin N Am. 1995;3(3):399-411. 
12. Walker MF, Souza SP, Dumoulin CL. Quantitative flow measurement in phase contrast MR angiography. J Comput Assist Tomogr. 1988;12(2):304-13.

13. Bakker CJ, Hoogeveen RM,Viergever MA. Construction of a protocol for measuring blood flow by two-dimensional phase-contrast MRA. J Magn Reson Imaging. 1999;9(1):119-27.

14. Leiner T, Nijenhuis RJ, Maki JH, et al. Use of a three-station phased array coil to improve peripheral contrast-enhanced magnetic resonance angiography. J Magn Reson Imaging. 2004;20(3):417-25.

15. Mohajer K, Zhang H, Gurell D, et al. Superficial femoral artery occlusive disease severity correlates with MR cine phase-contrast flow measurements. J Magn Reson Imaging. 2006;23(3):355-60.

16. Prakash A, Garg R, Marcus EN, et al. Faster flow quantification using sensitivity encoding for velocity-encoded cine magnetic resonance imaging: in vitro and in vivo validation. J Magn Reson Imaging. 2006;24(3):676-82.

17. Hanley JA, McNeil BJ. A method of comparing the areas under receiver operating characteristic curves derived from the same cases. Radiology. 1983;148(3):839-43.

18. Hirsch AT, Haskal ZJ, Hertzer NR, et al. ACC/AHA 2005 Practice Guidelines for the management of patients with peripheral arterial disease (lower extremity, renal, mesenteric, and abdominal aortic): a collaborative report from the American Association for Vascular Surgery/Society for Vascular Surgery, Society for Cardiovascular Angiography and Interventions, Society for Vascular Medicine and Biology, Society of Interventional Radiology, and the ACC/AHA Task Force on Practice Guidelines (Writing Committee to Develop Guidelines for the Management of Patients With Peripheral Arterial Disease): endorsed by the American Association of Cardiovascular and Pulmonary Rehabilitation; National Heart, Lung, and Blood Institute; Society for Vascular Nursing; TransAtlantic Inter-Society Consensus; and Vascular Disease Foundation. Circulation. 2006;113(11):e463-654.

19. Creager MA. Clinical assessment of the patient with claudication: the role of the vascular laboratory. Vasc Med. 1997;2(3):231-7.

20. Lindner JR, Womack L, Barrett EJ, et al. Limb stress-rest perfusion imaging with contrast ultrasound for the assessment of peripheral arterial disease severity. JACC Cardiovasc Imaging. 2008; 1(3):343-50. 


\section{ChAPTER 3}

21. Stein R, Hriljac I, Halperin JL, et al. Limitation of the resting ankle-brachial index in symptomatic patients with peripheral arterial disease. Vasc Med. 2006;11(1):29-33.

22. Al-Qaisi M, Nott DM, King $\mathrm{DH}$, et al. Ankle brachial pressure index (ABPI): An update for practitioners. Vasc Health Risk Manag. 2009;5:833-41.

23. Wu WC, Wang J, Detre JA, et al. Hyperemic flow heterogeneity within the calf, foot, and forearm measured with continuous arterial spin labeling MRI. Am J Physiol Heart Circ Physiol. 2008;294(5):H2129-36.

24. Burchert W, Schellong S, van den Hoff J, et al. Oxygen-15-water PET assessment of muscular blood flow in peripheral vascular disease. J Nucl Med. 1997;38(1):93-8.

25. Bragadeesh T, Sari I, Pascotto M, et al. Detection of peripheral vascular stenosis by assessing skeletal muscle flow reserve. J Am Coll Cardiol. 2005;45(5):780-5.

26. Peetrons P. Ultrasound of muscles. Eur Radiol. 2002;12(1):35-43.

27. Chloros GD, Smerlis NN, Li Z, et al. Noninvasive evaluation of upperextremity vascular perfusion. J Hand Surg Am. 2008;33(4):591-600.

28. Weber MA, Krix M, Delorme S. Quantitative evaluation of muscle perfusion with CEUS and with MR. Eur Radiol. 2007;17(10):2663-74.

29. Ludman PF, Volterrani M, Clark AL, et al. Skeletal muscle blood flow in heart failure measured by ultrafast computed tomography: validation by comparison with plethysmography. Cardiovasc Res. 1993;27(6):1109-15.

30. Duet M, Virally M, Bailliart O, et al. Whole-body (201)Tl scintigraphy can detect exercise lower limb perfusion abnormalities in asymptomatic diabetic patients with normal Doppler pressure indices. Nucl Med Commun. 2001;22(9):949-54.

31. Ament W, Lubbers J, Rakhorst G, et al. Skeletal muscle perfusion measured by positron emission tomography during exercise. Pflugers Arch. 1998;436(5):653-8.

32. Fleischer AC, Niermann KJ, Donnelly EF, et al. Sonographic depiction of microvessel perfusion: principles and potential. J Ultrasound Med. 2004;23(11):1499-506.

33. Bishara RA, Taha W, Alfarouk MO, et al. Duplex detected ankle peak systolic velocity: a new parameter for the assessment of degree of peripheralischemia. Int Angiol. 2004;23(4):368-72.

34. Hoogeveen RM, Bakker CJ, Viergever MA. MR phase-contrast flow measurement with limited spatial resolution in small vessels: value of modelbased image analysis. Magn Reson Med. 1999;41(3):520-8. 
35. Machida $\mathrm{H}$, Komori $\mathrm{Y}$, Ueno $\mathrm{E}$, et al. Accurate measurement of pulsatile flow velocity in a small tube phantom: comparison of phase-contrast cine magnetic resonance imaging and intraluminal Doppler guidewire. Jpn J Radiol. 2010;28(8):571-7. 



\section{CHAPTER 4}

\section{Functional MRI in peripheral arterial disease:}

\section{Arterial peak flow versus ankle-brachial index}

B. Versluis, P.J. Nelemans, R. Brans, J.E. Wildberger, G.W.H. Schurink, T. Leiner, W.H. Backes

Published at PLoS One, 2014:

Versluis B, Nelemans PJ, Brans R, et al. Functional MRI in Peripheral Arterial Disease: Arterial Peak Flow versus Ankle-Brachial Index. PLoS One. 2014;9(2):e88471 


\section{Abstract}

\section{Objectives}

The purpose of this study was to compare the success rate of successful arterial peak flow (APF) and ankle-brachial index (ABI) measurements in patients with suspected or known peripheral arterial disease (PAD). In addition the discriminative capabilities for disease severity of the APF and ABI measurements were compared.

\section{Materials and Methods}

183 patients with varying degrees of PAD were included. All subjects underwent $A B I$ measurements and vectorcardiographysynchronized quantitative 2D cine MR phase-contrast imaging of the popliteal artery to determine APF. Proportions of patients with successful $A P F$ and $A B I$ measurements were compared and the discriminative capability was evaluated to correctly classify disease severity of both methods by means of receiver-operator characteristic (ROC) curves and the corresponding areas under the curve (AUC).

\section{Results}

APF was successfully measured in $91 \%$ of the patients, whereas the $A B I$ could be determined in $71 \%$ of the patients $(p<0.01)$. Success rates of $A P F$ and $A B I$ were significantly higher in patients with intermittent claudication (95\% and $80 \%$, respectively) than in patients with critical ischemia ( $87 \%$ and $62 \%$, respectively). The AUCs for APF and ABI measurements were 0.74 and 0.75 , respectively, which were not significantly different $(p=0.90)$. 


\section{Conclusions}

Compared to the assessment of PAD severity with $A B I$, the success rate of MRI-based APF measurements is $20 \%$ higher, with similar discriminatory capacity for disease severity. Therefore, APF is a valuable measure to assess PAD severity in patients in whom ABI cannot be reliably determined, and is especially convenient in patients already scheduled to undergo MR angiography. 


\section{Introduction}

Peripheral arterial disease (PAD) is a highly prevalent condition in industrialized societies $(1,2)$, affecting up to $7 \%$ of the general population over the age of 70 years (2). The diagnosis of PAD is based on the typical clinical history, physical examination with palpation of pedal pulse and by measuring the ankle-brachial index (ABI) (3-6). The $A B I$ is a fast, widely available and cost-effective test for this purpose (7-12). Sensitivity of $A B I$ measurements for diagnosing PAD is high and the $A B I$ can be used to determine the severity and progression of PAD over time (11,13-15). Unfortunately, $A B I$ measurements also suffer from certain limitations such as inability to reliably acquire the $A B I$ in many patients with stiff and uncompressible ankle arteries due to severe arterial wall calcifications and poor interobserver variability (16-18). For example, studies have shown that the $A B I$ cannot be measured in at least $5-10 \%$ of the diabetic population $(12,15,18-23)$.

In addition to $A B I$ measurements, diagnostic (imaging) tools such as duplex ultrasonography (DUS), magnetic resonance angiography (MRA), computed tomography angiography (CTA) or invasive angiography are available for diagnosis, treatment planning and longitudinal follow-up of patients with PAD (2). Except DUS, all of these imaging modalities are accurate for visualizing the vascular morphology (24-26), but generally do not provide functional information, which makes it difficult to quantify and evaluate hemodynamic consequences over time, as is easily done by $A B I$ measurements. MRI, however, can also be used to evaluate vascular function, for example by measuring arterial peak flow (APF) with quantitative MR phase-contrast imaging (PCl) (27). The APF is a highly reproducible $(27,28)$, fast and simple measurement, which is not hampered by uncompressible arteries, like in $A B I$ measurements. For those PAD patients scheduled to undergo a MRA examination and in whom the $A B I$ cannot be determined, the combination of morphologic MRA and functional vascular MRI might help in the objective assessment of vascular status and be helpful in the follow-up of PAD patients, especially as (due to the progressive character of PAD) many 
patients will repeatedly undergo MRA examinations during lifetime. APF measurements in that respect are most promising if the proportion of successful APF measurements is markedly higher as compared to ABI measurements in this group of patients with PAD.

Therefore, the purpose of this study was to compare the proportions of a large group of patients scheduled for MRA with successful APF and $A B I$ measurements. In addition, we determined the influence of disease severity (intermittent claudication versus critical ischemia), presence of diabetes mellitus and the location of arterial lesions on the success rates of APF and $A B I$ measurements. Also the discriminative capability for disease severity of the APF and $A B I$ measurements was determined.

\section{Materials and Methods}

\section{Study population}

This study was approved by the institutional medical ethics committee. Considering the retrospective nature of the study the ethics committee waived the need for informed consent. The APF is routinely measured at our institution in every patient scheduled for contrastenhanced MR angiography (CE-MRA) of the peripheral arteries. Therefore, 210 consecutive patients undergoing CE-MRA of the peripheral arteries at our institution as part of a clinical routine examination between January and June 2010 were considered suitable for analysis in this study. In 27 patients CE-MRA was performed to rule out deep venous thrombosis $(n=16)$, or as part of the pre-operative visualization of the vasculature in patients scheduled for reconstructive surgery, with the lower leg serving as donor site $(n=11)$. After exclusion of these 27 patients, who did not undergo $A B I$ measurements, 183 patients were further analyzed in this study. Patient characteristics are presented in table 1. Diabetes mellitus was present in $48 \%$ of all patients (table 1 ). In 17 patients the diabetic status could not be established definitively. These patients were referred to our institution for diagnostic purposes 


\section{CHAPTER 4}

only and no full clinical history was present. These patients were excluded from relevant analysis. Distinction between intermittent claudication and critical ischemia was based on clinical symptoms as recorded by the referring vascular surgeon and retrieved from patient records.

\begin{tabular}{lccc}
\hline & $\begin{array}{c}\text { Intermittent claudi- } \\
\text { cation }\end{array}$ & Critical ischemia & All patients \\
\hline Patients & 94 & 89 & 183 \\
Males (\%) / Females (\%) & $58(62 \%) / 36(38 \%)$ & $45(51 \%) / 44(49 \%)$ & $103(56 \%) / 80(44 \%)$ \\
Age (years) & $64 \pm 12$ & $69 \pm 11$ & $67 \pm 12$ \\
Diabetics (\%) & $42(48 \%)^{\mathrm{a}}$ & $38(48 \%)^{\mathrm{b}}$ & $80(48 \%)$ \\
APF (mL/s) & $5.8 \pm 3.1$ & $3.9 \pm 2.8^{*}$ & $4.9 \pm 3.1$ \\
ABI & $0.74 \pm 0.22$ & $0.52 \pm 0.24^{*}$ & $0.65 \pm 0.25$ \\
\hline
\end{tabular}

Table 1. Patient characteristics.

Values are presented as mean \pm SD. APF, arterial peak flow; $A B I$, ankle-brachial index.

a Diabetic status was confirmed in 87 out of 94 patients. bDiabetic status was confirmed in 79 out of 89 patients.

" $\mathrm{p}<0.01$.

\section{MRI protocol}

All patients underwent quantitative cine $\mathrm{PCl}$ flow measurement of the popliteal artery (PA) to obtain flow wave forms, as required to determine the APF. PCI was preceded by a three-station CE-MRA protocol of the peripheral arteries. A schematic overview of the scan protocol is given in figure 1. All examinations were performed on a 1.5-T MRI system (Intera, Philips Medical Systems, Best, The Netherlands). Patients were imaged in supine position and had been in this position for approximately 30 minutes before the flow measurement was started. During this time the CE-MRA was acquired, using a fixed dose of $10 \mathrm{~mL}$ gadofosveset trisodium (Ablavar ${ }^{\circ}$, Lantheus Medical Imaging, Billerica, MA) as contrast agent. 


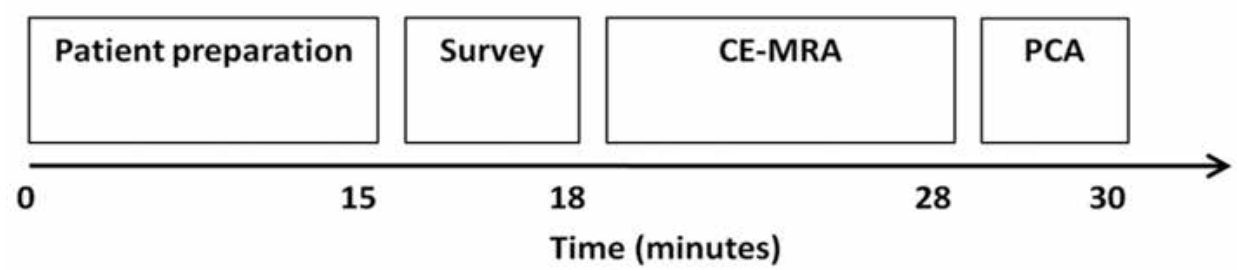

Figure 1. Overview of imaging protocol and the approximate duration of each acquired sequence. Contrast-enhanced MR angiography (CE-MRA) was combined with phasecontrast imaging (PCl) flow MRI. Total MRI examination time, including patient positioning, table movements and MRI scans, was approximately 35 minutes.

MR angiography. A three-station 3D gradient-echo (fast field echo) MRA sequence was performed as previously described $(25,29)$. Acquisition parameters were as follows: TR $4.8 \mathrm{~ms}$, TE $1.45 \mathrm{~ms}$, flip angle $40^{\circ}$, FOV $470 \mathrm{~mm}$, matrix 480 , and voxel dimensions (reconstructed) $0.92 \times 0.92 \times 1.20 \mathrm{~mm}$. Prior to contrast agent administration, a non-enhanced 'mask' image data set was acquired with exactly the same acquisition parameters as the CE-MRA, enabling background tissue suppression by image subtraction.

Flow MRI. For quantitative cine phase-contrast imaging we used a 2D gradient-echo (fast field echo) scan technique with the following acquisition parameters: TR $9.7 \mathrm{~ms}$, TE $5.8 \mathrm{~ms}$, flip angle $30^{\circ}$, FOV 380 $\mathrm{mm}$, matrix 384, and reconstructed voxel dimensions of $0.99 \times 0.99 \times$ $6.00 \mathrm{~mm}$. Fifteen dynamic phases were acquired to obtain the waveform that spanned the cardiac cycle. The phase encoding velocity was set to $100 \mathrm{~cm} / \mathrm{s}$ in the craniocaudal direction $(27,30)$. Vector cardiography (VCG) triggering was used for retrospective cardiac synchronization. Parallel imaging (sensitivity encoding, SENSE) was applied to reduce scan time (SENSE acceleration factor 2 in the anterior-posterior direction) (31). At a mean heart rate of 60 beats per minute, nominal acquisition time was 1 minute.

\section{Angiographic reading}

All angiographic datasets were analyzed by one well-trained radiologist with over 5 years of experience in CE-MRA of the peripheral 


\section{CHAPTER 4}

vasculature. For this study, CE-MRA data sets were used to determine whether or not obstructive arterial lesions were present within the peripheral vascular tree of most symptomatic extremity.

\section{Flow analysis}

Modulus and phase images were reconstructed from the cine phase-contrast data. A quantitative flow analysis package (QFlow) included with the software release (R1 1.4.14, Philips Medical Systems) of the MRI hardware was used for analysis of the flow data directly after the acquisition. Using this software, a region of interest (ROI) covering the entire visible cross-section of the artery of interest was accurately drawn manually using a reconstructed modulus image during peak systole and then automatically propagated to the remaining cardiac phases using an active contour algorithm. Although the peak systolic phase is sufficient to calculate the APF, ROI's were propagated to all cardiac phases to obtain flow wave forms, which were visually analyzed to reassure the chosen cardiac phase indeed was at peak systole and to detect possible aliasing effects directly after the acquisition. If detected, the measurement was repeated with sufficient higher phase encoding velocity.

APF was preferred over mean flow for analysis, as peak flow is known to be more reproducible and may exhibit large differences between patients and healthy controls (30). Given the high inter-reader reproducibility of the APF, all flow measurements were analyzed by a single blinded, well-experienced radiologist (27).

\section{$\mathrm{ABI}$ measurements}

$A B I$ data were retrieved from patient records. All $A B I$ measurements were performed by well-trained operators at our vascular function laboratory, using an automated Doppler system (Nicolet VasoGuard, VIASYS healthcare, Madison, WI). If the ABI could not be determined due to arterial stiffness or other circumstances, this was mentioned in the report by the operators. The $A B I$ was acquired within 2 months prior to 
the MRI exam for all patients, and patients did not receive invasive treatment between the two exams.

\section{Statistical analysis}

For statistical analysis only APF and $A B I$ data obtained in the (most) symptomatic leg were used. If both legs were equally symptomatic, the right leg was analyzed. Statistical analysis was performed with commercially available statistical software (SPSS 16.0, SPSS Inc., Chicago, IL). The proportions of patients with successful APF and ABI measurements were compared and tested for statistical significance using McNemar's test for paired proportions. A chi-square test was used to test between group differences in proportions and a

$t$-test for independent samples was used to test between-group differences in continuous variables.

The ability of APF and ABI measurements to discriminate between patients with intermittent claudication and critical ischemia was compared using receiver operating characteristic (ROC) curves and the area under the curve (AUC). Differences in AUCs were tested for statistical significance using the method described by Hanley et al, which accounts for the fact that AUCs were derived from the same sample of patients (32). In all analysis, $\mathrm{p}$-values $<0.05$ were considered to indicate statistical significance.

\section{Results}

\section{APF and $A B I$}

The APF measured in the (most) symptomatic leg as well as $A B I$ is presented in table 1. Typical flow waveforms of patients with intermittent claudication and critical ischemia are presented in figure 2 . Both the APF and $A B I$ were significantly lower in patients with critical ischemia compared to patients with intermittent claudication ( $p<0.01$ ). In all patients with obstructive arterial lesions below the level of the PA 


\section{CHAPTER 4}

regardless the severity of the clinical symptoms (i.e. both patients with intermittent claudication and critical ischemia; $n=103$ ), APF was significantly lower $(3.9 \pm 2.7 \mathrm{~mL} / \mathrm{s})$ compared to patients without arterial lesions below the PA $(5.2 \pm 3.3 \mathrm{~mL} / \mathrm{s} ; \mathrm{p}<0.01)$. Comparable differences were found for the $\mathrm{ABI}$ measurements $(0.56 \pm 0.23$ and $0.68 \pm$ 0.25 , respectively; $\mathrm{p}<0.01$ ).

A

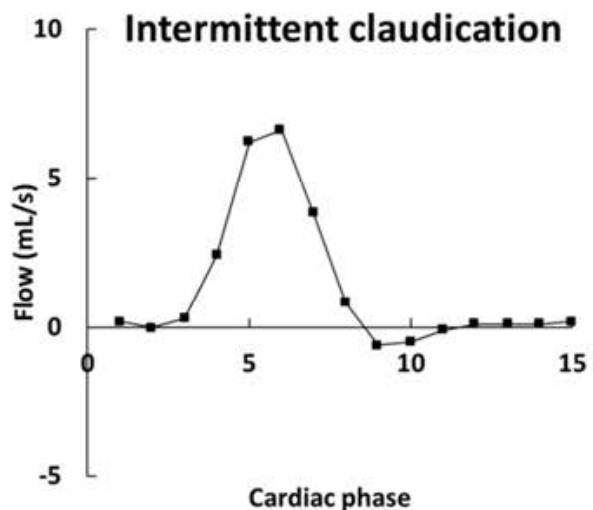

B

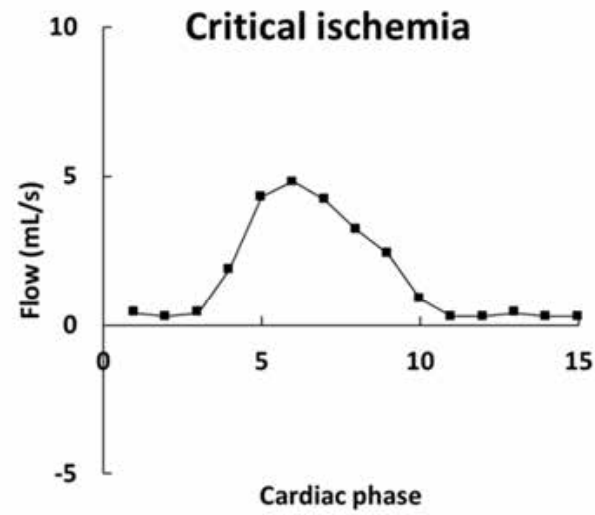

Figure 2. Flow waveforms in patients with intermittent claudication (A) and critical ischemia ( B). Note the bi-phasic flow waveform and a higher peak flow value in intermittent claudication compared to the mono-phasic flow waveform with a lower peak flow value in critical ischemia.

\section{Success rates of APF and $A B I$ measurements}

Success rates of APF and ABI measurements are presented in tables 2 and 3 . Success rates of APF measurements were significantly higher compared to $A B I$ measurements (91\% versus $71 \%, p<0.01$ ). APF and $A B I$ measurements were successful with both methods in 121 (66\%) patients, whereas in $96 \%$ of the patients either APF or ABI measurements were successful. In 45 (25\%) patients only the APF could be determined and in 9 (5\%) patients only the $A B I$ could be determined. 


\begin{tabular}{llll}
\hline & Successful APF & Successful ABI & p-value \\
\hline All patients & $91 \%(166 / 183)$ & $71 \%(130 / 183)$ & $<0.01$
\end{tabular}

Disease severity:

$\begin{array}{llll}\text { IC } & 95 \%(89 / 94) & 80 \%(75 / 94) & <0.01 \\ \mathrm{Cl} & 87 \%(77 / 89) & 62 \%(55 / 89) & <0.01\end{array}$

Diabetes mellitusa

Yes

$95 \%(76 / 80)$

$69 \%(55 / 80)$

$<0.01$

No

$90 \%(77 / 86)$

$71 \%(61 / 86)$

$<0.01$

Obstruction below PA

Yes

$88 \%(91 / 103)$

$68 \%(70 / 103)$

$<0.01$

No

$96 \%(72 / 75)$

$78 \%(59 / 75)$

$<0.01$

Table 2. Success rates of APF and ABI measurements.

APF, arterial peak flow; $A B I$, ankle-brachial index; IC, intermittent claudication; $\mathrm{Cl}$, critical ischemia, PA, popliteal artery. a Diabetic status could be determined in 166 out of 183 patients. bIn 5 out of 183 the PA or main arteries of the lower leg could not be assessed due to severe image artifacts caused by metallic knee prostheses.

\section{Factors influencing measurement success rate}

There were large differences in the proportions of subjects in whom APF and $A B I$ could be obtained between patients with intermittent claudication and critical ischemia (table 2 , disease severity). The success rates were highest in patients with intermittent claudication $(p=0.06$ and $p=0.01$ for APF and ABI measurements, respectively) compared to those with critical ischemia.

As can be seen in table 3, approximately half of patients with diabetes mellitus in our population had intermittent claudication. No significant differences in success rates were found between patients with and without diabetes mellitus for both APF and ABI measurements ( $p=0.19$ and $p=0.64$ for APF and $A B I$ measurements, respectively). However, $A B I$ measurements were significantly more often successful in diabetic patients with intermittent claudication (table 3, 84\%) than in those with critical ischemia $(61 \%, p<0.01)$. No further significant 


\section{Chapter 4}

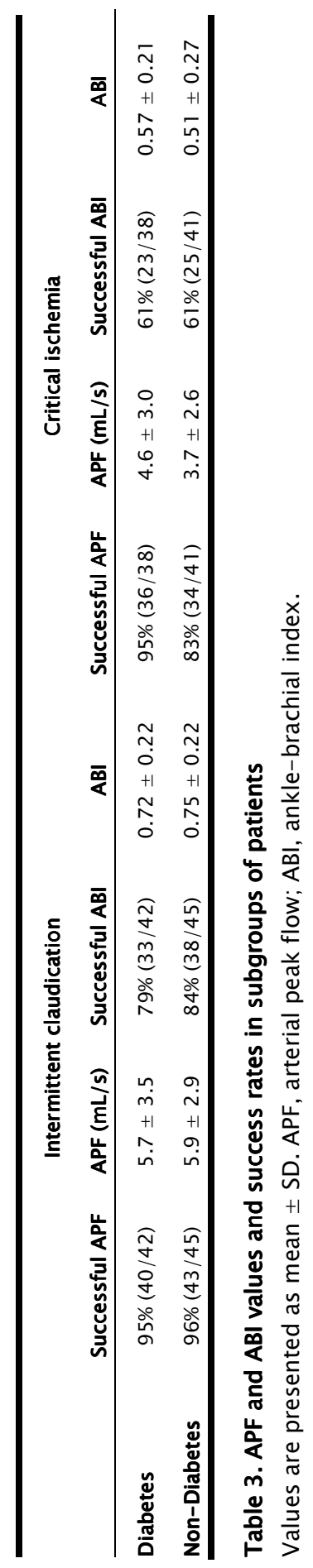


differences were found between the subgroups of diabetes mellitus and disease severity in table 3 .

Patients without arterial obstructive pathology below the level of the APF measurement showed a trend of higher success rates in APF $(p=0.07)$ and $A B I(p=0.11)$ measurements.

Main causes of failure of the APF measurements $(n=17,9 \%)$ were hardware failure $(n=7)$, occlusion of the PA $(n=7)$ and irregular heart rate $(n=1)$. In 2 patients the cause of failure remained unknown.

Main causes of failure for $A B I$ measurements ( $n=53,29 \%$ ) were non-compressible arteries due to arterial stiffness $(n=42)$ and presence of PAD-related ulcers or wounds, making it impossible to use a cuff to measure the systolic pressure at the level of the ankle $(n=7)$. In 4 patients no cause of failure was reported in the patient records.

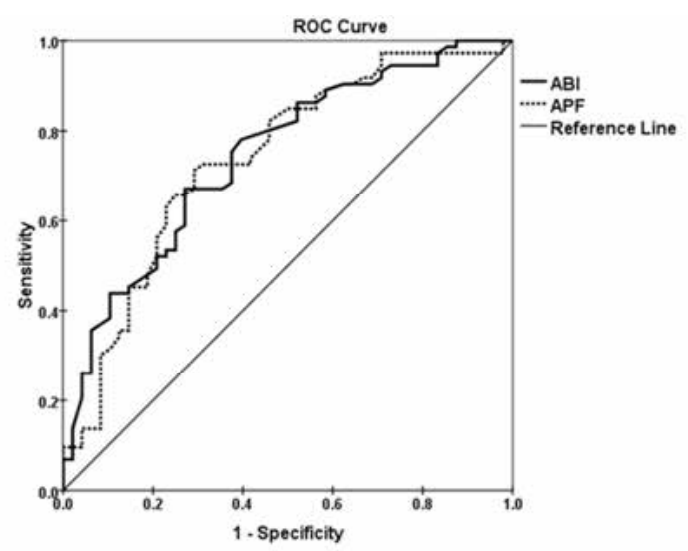

Figure 3. ROC curves for arterial peak flow (APF) and ankle-brachial index (ABI) in 122 patients with successful APF and ABI measurements. The curves show comparable discriminative capability for determination of disease severity (intermittent claudication versus critical ischemia) between APF and $A B I$ measurements.

\section{Discriminative capability}

Figure 3 shows ROC curves for both APF and $A B I$ measurements in 122 patients with intermittent claudication or critical ischemia, in whom both APF as well as $A B I$ measurement were successfully obtained. The AUC of the APF was $0.74 \quad(95 \% \quad$ confidence interval $(\mathrm{Cl}), 0.66-0.83)$ and $0.75(95 \% \mathrm{Cl}, 0.65-$ 0.83) for $A B I$, respectively, and were not statistically significant ( $p=0.90)$. 


\section{Discussion}

Objective assessment of vascular function is important in the diagnostic work-up and follow-up of patients with PAD. The most widely used measure for this purpose is the $A B I$. Although the $A B I$ has a high sensitivity to detect PAD, we found that the $A B I$ could not be determined in up to $29 \%$ of the patients included in this study. Alternative functional measurements would therefore be desirable to objectively characterize the peripheral vascular status in these patients. The results of this study show that popliteal arterial peak flow (APF), measured with MRI in clinical PAD patients scheduled for MRA, has a success rate of up to $91 \%$, which was significantly higher compared to $A B I$ measurements (71\%), with similar discriminative capability.

\section{Success rates}

$A B I$ measurements were unsuccessful in up to $29 \%$ of the patients. This is markedly worse than the $5-10 \%$ known from previous literature $(12,15,18)$. The most likely explanation for this discrepancy is the specific population for this study, which comprises only PAD patients referred for a clinical CE-MRA exam, rather than the general population of PAD patients. This means our population is likely to harbor more severe disease compared to patients with PAD who do not require a CEMRA for diagnosis or treatment planning.

Although in $91 \%$ of the patients functional information could be obtained by performing APF measurements, combined measurement of $A B I$ and APF resulted in a slight improvement to $96 \%(n=176)$ in the proportion of patients with usable functional information. Therefore, APF measurements should not be considered as a replacement for ABI measurements, but rather as a useful addition.

\section{Factors influencing success rate}

$A B I$ measurements could be determined more often in patients with intermittent claudication compared to patients with critical ische- 
mia. This can be explained by the higher number of patients with ulceration and tissue loss as well as the more pronounced arterial stiffness in these patients with more severe state of PAD.

The presence of arterial lesions in the lower leg showed a trend towards lower success rates in both APF and ABI measurements. As far as the APF is concerned, this was due to the presence of popliteal occlusions, whereas for the $A B I$ measurements this was due to the presence of skin ulcera/wounds and more pronounced arterial stiffness. As the APF is measured in the PA, while the ABI is acquired at anklelevel, a direct comparison between quantitative measures might not be reliable, especially in patients with arterial lesions in the lower leg. However, analysis of the APF data revealed significantly lower flow values for patients with significant arterial lesions in the lower leg. Therefore, flow measurements of the PA seem to be influenced by obstructive lesions in both in- and outflow trajectories. These results were concordant with the $A B I$ data.

Another factor associated with a trend towards lower success rates of both APF and ABI measurements was the presence of arterial lesions at the level of and below the knee. In this patient group, APF could not be determined in case of popliteal occlusions. ABI measurements on the other hand, were hard to obtain in the patients with ischemic wounds and more pronounced arterial stiffness.

\section{Discriminative capability}

Determination of APF with $\mathrm{PCl}$ has been shown to have good reproducibility and discriminative capabilities before (27). In this study we found that APF in patients with critical ischemia was significantly lower compared to patients with intermittent claudication. AUCs of the ROC curves for both APF and $A B I$ measurements were comparable. This indicates that there is no relevant difference in discriminative capability of $A P F$ and $A B I$ measurements to distinguish PAD patients with intermittent claudication from those with critical ischemia. Therefore, APF measurements are a reliable alternative to $A B I$ measurements in patients in whom the ABI cannot be determined. 


\section{APF}

APF, defined as the maximum systolic flow, is an attractive arterial flow measure. Although quantitative MRI-based flow measurements can also be used to measure mean flow, we chose to measure the APF, only. This is an important determinant of the systolic blood pressure (28), that shows relatively large differences between PAD patients and control subjects and is more reproducible than the mean flow (30).

For this study only patients scheduled for MRA were included. Although a selected population was used, as far as APF measurements are concerned this is clinically the most relevant patient population, as MRIbased APF measurements are not (yet) suitable for screening purposes due to the limited availability of MRI systems or magnet time and associated costs. Also, as the selected study population comprised consecutive patients undergoing CE-MRA, it is a clinically representative group as far as disease severity and presence of diabetes mellitus is concerned. Nevertheless, this implies that current findings do not necessarily apply for all PAD patients. We do see an increasing number of PAD patients undergoing multiple MRA examinations during lifetime for treatment planning and/or therapy monitoring. A fast and simple functional MRI measurement, such as the APF, is therefore a valuable addition to MRA in order to objectively quantify the vascular status and to monitor the progression of PAD over time, especially in patients in whom the $A B I$ cannot reliably be determined.

This study and our previous work (27) demonstrate that the APF is a reproducible measure that can be acquired in almost every PAD patient scheduled for MRA and can discriminate between non-PAD patients and PAD patients with intermittent claudication or critical ischemia. Further research will be required to determine the most suitable location to assess the APF, to determine normative values and to demonstrate the clinical relevance of APF, ie. Its influence on therapy and clinical course of PAD. 


\section{Conclusion}

Compared to assessment of PAD severity with $A B I$, the success rate of APF measurements is $20 \%$ higher, with similar discriminatory capacity for disease severity. Therefore, APF is a valuable ancillary method to assess PAD severity in patients referred for MRA, especially if $A B I$ cannot be reliably determined. 


\section{References}

1. Hirsch AT, Haskal ZJ, Hertzer NR, et al. ACC/AHA 2005 guidelines for the management of patients with peripheral arterial disease (lower extremity, renal, mesenteric, and abdominal aortic): executive summary a collaborative report from the American Association for Vascular Surgery/Society for Vascular Surgery, Society for Cardiovascular Angiography and Interventions, Society for Vascular Medicine and Biology, Society of Interventional Radiology, and the ACC/AHA Task Force on Practice Guidelines (Writing Committee to Develop Guidelines for the Management of Patients With Peripheral Arterial Disease) endorsed by the American Association of Cardiovascular and Pulmonary Rehabilitation; National Heart, Lung, and Blood Institute; Society for Vascular Nursing; TransAtlantic Inter-Society Consensus; and Vascular Disease Foundation. J Am Coll Cardiol. 2006;47(6): 1239-312.

2. Norgren L, Hiatt WR, Dormandy JA, et al. Inter-Society Consensus for the Management of Peripheral Arterial Disease (TASC II). J Vasc Surg. 2007;45 Suppl S:S5-67.

3. Gerhard-Herman M, Gardin JM, Jaff M, et al. Guidelines for noninvasive vascular laboratory testing: a report from the American Society of Echocardiography and the Society for Vascular Medicine and Biology. Vasc Med. 2006;11(3):183-200.

4. Creager MA. Clinical assessment of the patient with claudication: the role of the vascular laboratory. Vasc Med. 1997;2(3):231-7.

5. Andersen CA. Noninvasive assessment of lower extremity hemodynamics in individuals with diabetes mellitus. J Vasc Surg. 2010;52(3 Suppl):76S80 s.

6. Begelman SM, Jaff MR. Noninvasive diagnostic strategies for peripheral arterial disease. Cleve Clin J Med. 2006;73 Suppl 4:S22-9.

7. Aslam F, Haque A, Foody J, et al. Peripheral arterial disease: current perspectives and new trends in management. South Med J. 2009;102(11):1141-9.

8. McDermott MM, Criqui MH, Greenland $\mathrm{P}$, et al. Leg strength in peripheral arterial disease: associations with disease severity and lower-extremity performance. J Vasc Surg. 2004;39(3):523-30. 
9. McDermott MM, Greenland P, Liu K, et al. Leg symptoms in peripheral arterial disease: associated clinical characteristics and functional impairment. JAMA. $2001 ; 286(13): 1599-606$.

10. Resnick HE, Lindsay RS, McDermott MM, et al. Relationship of high and low ankle brachial index to all-cause and cardiovascular disease mortality: the Strong Heart Study. Circulation. 2004;109(6):733-9.

11. Doobay AV, Anand SS. Sensitivity and specificity of the ankle-brachial index to predict future cardiovascular outcomes: a systematic review. Arterioscler Thromb Vasc Biol. 2005;25(7):1463-9.

12. Potier L, Abi Khalil C, Mohammedi K, et al. Use and Utility of Ankle Brachial Index in Patients with Diabetes. Eur J Vasc Endovasc Surg. 2010.

13. Dachun $X$, Jue L, Liling $Z$, et al. Sensitivity and specificity of the ankle-brachial index to diagnose peripheral artery disease: a structured review. Vasc Med. 2010;15(5):361-9.

14. Guo X, Li J, Pang W, et al. Sensitivity and specificity of ankle-brachial index for detecting angiographic stenosis of peripheral arteries. Circ J. 2008;72(4):605-10.

15. Aboyans V, Ho E, Denenberg JO, et al. The association between elevated ankle systolic pressures and peripheral occlusive arterial disease in diabetic and nondiabetic subjects. J Vasc Surg. 2008;48(5):1197-203.

16. van Langen $H$, van Gurp J, Rubbens $L$. Interobserver variability of anklebrachial index measurements at rest and post exercise in patients with intermittent claudication. Vasc Med. 2009;14(3):221-6.

17. Allen J, Oates CP, Henderson J, et al. Comparison of lower limb arterial assessments using color-duplex ultrasound and ankle/brachial pressure index measurements. Angiology. 1996;47(3):225-32.

18. Stein R, Hriljac I, Halperin JL, et al. Limitation of the resting ankle-brachial index in symptomatic patients with peripheral arterial disease. Vasc Med. 2006;11(1):29-33.

19. Brooks B, Dean R, Patel S, et al. TBI or not TBI: that is the question. Is it better to measure toe pressure than ankle pressure in diabetic patients? Diabet Med. 2001;18(7):528-32.

20. Giachelli CM. Vascular calcification mechanisms. J Am Soc Nephrol. 2004;15(12):2959-64.

21. Goss DE, de Trafford J, Roberts VC, et al. Raised ankle/brachial pressure index in insulin-treated diabetic patients. Diabet Med. 1989;6(7):576-8. 


\section{CHAPTER 4}

22. London GM, Guerin AP, Marchais SJ, et al. Arterial media calcification in end-stage renal disease: impact on all-cause and cardiovascular mortality. Nephrol Dial Transplant. 2003;18(9):1731-40.

23. Raines JK, Darling RC, Buth J, et al. Vascular laboratory criteria for the management of peripheral vascular disease of the lower extremities. Surgery. 1976;79(1):21-9.

24. de Vries M, de Koning PJ, de Haan MW, et al. Accuracy of semiautomated analysis of 3D contrast-enhanced magnetic resonance angiography for detection and quantification of aortoiliac stenoses. Invest Radiol. 2005;40(8):495-503.

25. de Vries M, Nijenhuis RJ, Hoogeveen RM, et al. Contrast-enhanced peripheral MR angiography using SENSE in multiple stations: feasibility study. J Magn Reson Imaging. 2005;21(1):37-45.

26. Leiner T, Kessels AG, Nelemans PJ, et al. Peripheral arterial disease: comparison of color duplex US and contrast-enhanced MR angiography for diagnosis. Radiology. 2005;235(2):699-708.

27. Versluis B, Backes WH, van Eupen MG, et al. Magnetic resonance imaging in peripheral arterial disease: reproducibility of the assessment of morphological and functional vascular status. Invest Radiol. 2011;46(1):1124.

28. Bishara RA, Taha W, Alfarouk MO, et al. Duplex detected ankle peak systolic velocity: a new parameter for the assessment of degree of peripheralischemia. Int Angiol. 2004;23(4):368-72.

29. Leiner T, Nijenhuis RJ, Maki JH, et al. Use of a three-station phased array coil to improve peripheral contrast-enhanced magnetic resonance angiography. J Magn Reson Imaging. 2004;20(3):417-25.

30. Mohajer K, Zhang H, Gurell D, et al. Superficial femoral artery occlusive disease severity correlates with MR cine phase-contrast flow measurements. J Magn Reson Imaging. 2006;23(3):355-60.

31. Prakash A, Garg R, Marcus EN, et al. Faster flow quantification using sensitivity encoding for velocity-encoded cine magnetic resonance imaging: in vitro and in vivo validation. J Magn Reson Imaging. 2006;24(3):676-82.

32. Hanley JA, McNeil BJ. A method of comparing the areas under receiver operating characteristic curves derived from the same cases. Radiology. $1983 ; 148(3): 839-43$. 


\section{CHAPTER 5}

Reference values for peripheral arterial peak

flow in patients with peripheral arterial disease: Assessment by phase-contrast magnetic resonance flow imaging

B. Versluis, W.H. Backes, P.J. Nelemans, R. Brans, J.E. Wildberger, G.W.H. Schurink, T. Leiner 


\section{CHAPTER 5}

\section{Abstract}

\section{Objectives}

The aim of this study was to provide reference values for popliteal arterial peak flow (APF) and to assess the influence of disease severity, and presence of diabetes mellitus in patients with peripheral arterial disease (PAD).

\section{Materials and Methods}

239 patients with varying degrees of PAD, 45 patients without PAD and 28 healthy subjects were included. All subjects underwent quantitative 2D cine MR phase-contrast velocity imaging at the level of popliteal artery (PA). APF values of PAD patients were compared with a reference population consisting of non-PAD subjects. Furthermore, average APF values were compared between subgroups of PAD patients with intermittent claudication and critical ischemia and with or without diabetes mellitus. The ability to discriminate between PAD and non-PAD subjects was evaluated by using a receiver operating characteristic (ROC) curve with corresponding area under the curve (AUC).

\section{Results}

Average APF values in PAD patients (mean \pm SD, $4.6 \pm 3.0 \mathrm{~mL} / \mathrm{s}$ ) were $58 \%$ lower compared to non-PAD subjects $(11.0 \pm 3.5 \mathrm{~mL} / \mathrm{s}, \mathrm{p}<$ $0.01)$. APF was significantly lower in PAD patients with critical ischemia $(4.1 \pm 3.2 \mathrm{~mL} / \mathrm{s})$ compared to PAD patients with intermittent claudication $(5.1 \pm 2.9 \mathrm{~mL} / \mathrm{s}, \mathrm{p}=0.02)$. The presence of diabetes mellitus did not appear to be a factor of significant influence. The ability of APF to discriminate between patients with and without PAD, expressed as AUC, was 0.90 (95\% Cl: 0.86-0.95). 


\section{Conclusions}

This study provides MRI derived reference values for APF in patients with clinically manifest PAD as well as a control sample of patients referred for non-PAD indications and healthy subjects. These reference values may aid in objective functional quantification of PAD severity and follow-up after intervention. 


\section{Introduction}

The diagnosis of peripheral arterial disease (PAD) is made based on the typical pattern of complaints, palpation of pedal pulses and measurement of the ankle-brachial index $(A B I)(1,2)$. Non-invasive imaging is typically only requested when the need for invasive intervention arises. A frequently used modality in the pre-interventional workup is magnetic resonance angiography (MRA). There is a large body of evidence that MRA is a highly accurate non-invasive diagnostic imaging method for the assessment of the location and severity of obstructions in peripheral conduit arteries in patients with PAD (2-5).

Currently used MR imaging protocols are exclusively focused on morphological evaluation of the vascular tree. There remains a need, however, for a fast and simple functional measure that can serve as a proxy for the hemodynamic severity of vascular obstructions visualized with MRA. For this purpose, MRI derived arterial peak flow (APF) may be a simple and valuable addition to standard MRA protocols $(6,7)$. Although numerous other non-invasive functional diagnostic tools are available for this purpose such as ABI, duplex ultrasonography and laser Doppler measurements (8-11), APF measurements have the advantage of being relatively fast and reproducible and can easily be added to existing MRA-protocols $(6,7,12-14)$. This is of interest as the aforementioned conventional functional measures might not always yield reliable results, for instance in the presence of non-compressible arteries in the case of the $\mathrm{ABI}$, or a poor acoustic window in the case of duplex ultrasonography.

The simplest way to measure MRI derived APF is by twodimensional (2D) cine phase contrast imaging. Previous studies have demonstrated good reproducibility of this method and lower APF values in PAD patients relative to healthy control subjects $(6,7)$. The $2 \mathrm{D}$ nature of the protocol enables fast imaging, but comes at the expense of anatomical coverage, as it is limited to interrogation of a single anatomical location at once. It is desirable, therefore, to identify a standard location in the peripheral vascular tree that combines high sensitivity 
with high specificity for detection of hemodynamically significant PAD. However, little is known at present about absolute APF values, both in healthy subjects, as well as in various categories of patients with PAD. Consequently, the aims of the current study were to establish reference values for 2D MRI derived resting APF in PAD patients. In addition, we evaluated the influence of disease severity (intermittent claudication versus critical ischemia), presence of diabetes mellitus and age upon the APF. To this end, we determined the APF at the level of the popliteal artery (PA) in a large group of PAD patients referred for lower extremity MRA, as well as a control sample of patients undergoing MRA of the peripheral vascular tree for non-PAD indications and healthy subjects. APF was measured at the level of the PA, as the PA is the most distally located large peripheral artery, reflecting all proximal obstructive arterial lesions.

\section{Materials and Methods}

\section{Study population}

259 PAD patients were referred for contrast-enhanced MR angiography (CE-MRA) of the peripheral arteries at our institution between January and December 2010. In 20 patients the APF could not be obtained at the level of the PA (table 3, chapter 3), these patients were excluded from this study. Otherwise there were no exclusion criteria. Therefore, 239 patients were analyzed in this study. Non-patients referred for CE-MRA of the peripheral arterial tree to rule out deep vein thrombosis or in the pre-operative workup for reconstructive surgery using lower extremity tissue flaps with no signs of PAD on CE-MRA and with successful APF measurements $(n=45)$, were used as reference in this study. Patient characteristics were retrieved from the patients' records and are summarized in table 1. The ethics committee waived the need for informed consent for patients, as flow measurements are part of the standard clinical imaging protocol at our institution. In addition, flow data were obtained in 28 healthy subjects. Non-PAD patients and healthy subjects were clustered together and referred to as non-PAD 


\section{ChAPTER 5}

subjects. The institutional medical ethics committee approved this part of the study and all volunteers provided signed informed consent prior to inclusion.

\begin{tabular}{lccc}
\hline & PAD patients & Non-PAD patients & Healthy subjects \\
\hline Number & 239 & 45 & 28 \\
Males (\%) / Females (\%) & $139(58 \%) / 100(42 \%)$ & $21(47 \%) / 24(53 \%)$ & $6(21 \%) / 22(79 \%)$ \\
Age ( years) & $66 \pm 13.5$ & $52 \pm 16.1^{*}$ & $24 \pm 7.5^{*}$ \\
IC/Cl & $128(54 \%) / 111(46 \%)$ & $0(0 \%)$ & $0(0 \%)$ \\
Diabetics (\%) & $106(52 \%)^{\mathrm{a}}$ & $5(14 \%)^{\mathrm{b}}$ & $0(0 \%)$ \\
\hline
\end{tabular}

Table 1. Patient characteristics.

Values are presented as mean \pm SD. PAD, peripheral arterial disease; IC, intermittent claudication; $\mathrm{Cl}$, critical ischemia.

* $\mathrm{p}<0.01$.

a Diabetic status could only be retrieved in 202 out of 239 PAD patients. bDiabetic status could only be retrieved in 35 out of 45 non-PAD patients.

\section{MRI protocol}

All patients underwent a three-station CE-MRA protocol of the peripheral arteries (15), followed by quantitative MR cine phase-contrast imaging flow measurements at the level of the popliteal artery. Flow data were used to obtain absolute APF values in PAD and non-PAD patients as well as healthy subjects (16). All examinations were performed using a 1.5-T MRI system (Intera, R1 1.4.14, Philips Healthcare, Best, The Netherlands). For signal reception we used a dedicated 12element phased-array peripheral vascular coil with a craniocaudal coverage of $128 \mathrm{~cm}$ (Philips Healthcare). Patients were imaged in supine position and had been in this position for approximately 30 minutes before flow measurements were started. Prior to flow measurements the CE-MRA examination was performed, using a fixed dose of $10 \mathrm{~mL}$ gadofosveset trisodium (Ablavar ${ }^{\circ}$, Lantheus Medical Imaging, Billerica, MA) as contrast agent.

Survey. A non-enhanced time-of-flight (TOF) scan of the pelvic, upper and lower leg station was performed to prescribe the imaging 
volumes of interest for morphological and functional imaging. A turbo field echo pulse sequence was used with a $180^{\circ}$ inversion prepulse to suppress stationary tissues. Thirty-one axial slices per station were acquired with 3.3-mm slice thickness and $11-\mathrm{mm}$ interslice gap, and an inferiorly concatenated saturation band was used to suppress signal from veins. The standard quadrature body coil was used for signal transmission and reception. To facilitate positioning of the 3D CE-MRA volumes maximum intensity projections (MIP) were generated in 3 orthogonal directions.

CE-MRA. A three-station 3D gradient-echo (fast field echo) MRA sequence was performed as previously described $(4,15)$. Acquisition parameters were as follows: TR $4.8 \mathrm{~ms}$, TE $1.45 \mathrm{~ms}$, flip angle $40^{\circ}$, FOV $470 \mathrm{~mm}$, matrix 480, and voxel dimensions (reconstructed) $0.92 \times 0.92$ $\times 1.20 \mathrm{~mm}^{3}$. Prior to contrast agent administration, a non-enhanced 'mask' image data set was acquired with exactly the same acquisition parameters as the CE-MRA, enabling background tissue suppression by image subtraction.

Flow MRI. For quantitative cine phase-contrast imaging we used a 2D gradient-echo (fast field echo) scan technique was used with the following acquisition parameters: TR $9.7 \mathrm{~ms}$, TE $5.8 \mathrm{~ms}$, flip angle $30^{\circ}$, FOV $380 \mathrm{~mm}$, matrix 384, and reconstructed voxel dimensions of $0.99 \times$ $0.99 \times 6.00 \mathrm{~mm}^{3}$. Fifteen dynamic phases were acquired during the cardiac cycle. Measurements were performed at the level of the P2 segment of the popliteal artery. The phase encoding velocity was set to standard values of $60 \mathrm{~cm} / \mathrm{s}$ in the craniocaudal direction $(6,16)$. Vector cardiography (VCG) triggering was used for retrospective cardiac synchronization. Parallel imaging was applied to reduce scan time (acceleration factor 2 in the anterior-posterior direction) (17). At a mean heart rate of 60 beats per minute, the nominal acquisition time of this sequence was 1 minute. Coronal and sagittal maximum intensity projected reconstructions of CE-MRA recordings were used for accurate angulation of the slice, perpendicular to the direction of the PA. 


\section{CHAPTER 5}

\section{CE-MRA}

For this study, CE-MRA data were used to determine the presence and severity of obstructive arterial lesions in PAD and to exclude presence of obstructive arterial lesions or arterial malformations in the group of non-PAD subjects. All CE-MRA datasets were analyzed by one well-trained radiologist with over 5 years of experience in MRA of the peripheral vasculature.

\section{Flow analysis}

Modulus and phase images were reconstructed from the cine flow data. A quantitative flow analysis package (QFlow) included with the software release (R1 1.4.14) of the MRI hardware was used for analysis of the flow data directly after the acquisition. Using this software, a region of interest (ROI) covering the entire visible cross-section of the artery of interest was manually drawn using a modulus image at peak systole and then automatically propagated to the remaining cardiac phases using an active contour algorithm. Although the peak systolic phase only is sufficient to calculate the APF, ROI's were propagated to the remaining cardiac phases to obtain flow wave forms, which were visually analyzed to reassure the chosen cardiac phase was indeed at peak systole and to detect possible aliasing effects. If aliasing artifacts were detected, the measurement was repeated with sufficiently higher phase encoding velocity.

\section{APF analysis}

APF was determined in the more symptomatic leg in patients with PAD and in the right leg in non-PAD subjects. If both legs were equally affected in patients with PAD, the right leg APF was used for analysis. Using CE-MRA data, patients with PAD were divided into two subgroups, i.e. patients with and without relevant arterial stenoses in the peripheral arterial tree. A relevant stenosis was defined as $>50 \%$ luminal narrowing in the common or external iliac or common or superficial femoral or popliteal arteries, independent of the extensiveness and location of the 
lesion. In addition, $>50 \%$ stenosis in at least two out of three main arteries of the lower leg were considered relevant. If the maximum stenosis grade anywhere in the peripheral arterial tree was $<50 \%$, these lesions were considered not to be hemodynamically relevant. A comparison of APF values was made between PAD patients with and without relevant stenoses in the peripheral arterial tree and non-PAD subjects as well as healthy controls. Furthermore, APF values in subgroups of PAD patients with intermittent claudication and critical ischemia as well as with or without diabetes mellitus were compared and the ability to discriminate between PAD ad non-PAD subjects was evaluated. In non-PAD subjects, the influence of gender and age on APF was evaluated.

\section{Statistical analysis}

Statistical analysis was performed with commercially available statistical software (SPSS 16.0, SPSS Inc., Chicago, IL) and STATA (11.2, StataCorp LP., Texas). Mean values between groups were tested for statistical significance using a $t$-test for independent samples. The ability of APF measurements to discriminate between PAD and non-PAD subjects was compared using receiver operating characteristic (ROC) curves and area-under-curve (AUC) values. In all analyses, $p$-values $<0.05$ were considered to indicate statistical significance.

\begin{tabular}{lcccccc}
\hline \multicolumn{3}{c}{ PAD patients } & \multicolumn{2}{c}{ Non-PAD subjects } \\
\multicolumn{2}{l}{ Relevant stenosis } & No relevant stenosis & & \\
APF $(\mathrm{mL} / \mathrm{s})$ & $\mathbf{n}$ & APF $(\mathrm{mL} / \mathrm{s})$ & $\mathbf{n}$ & APF $(\mathrm{mL} / \mathrm{s})$ & $\mathbf{n}$ \\
\hline $4.6 \pm 3.0$ & 218 & $8.7 \pm 3.8$ & 21 & $11.0 \pm 3.5$ & 73 \\
\hline
\end{tabular}

Table 2. Arterial peak flow in PAD and non-PAD subjects.

Values are presented as mean \pm SD. PAD, peripheral arterial disease; APF, arterial peak flow. 


\section{Results}

Relevant stenoses were present within the peripheral arterial tree in 218 out of 239 patients (91\%) with PAD according to CE-MRA, whereas no relevant stenoses were found in 21 (9\%) patients initially diagnosed with PAD (see table 2, chapter 4).
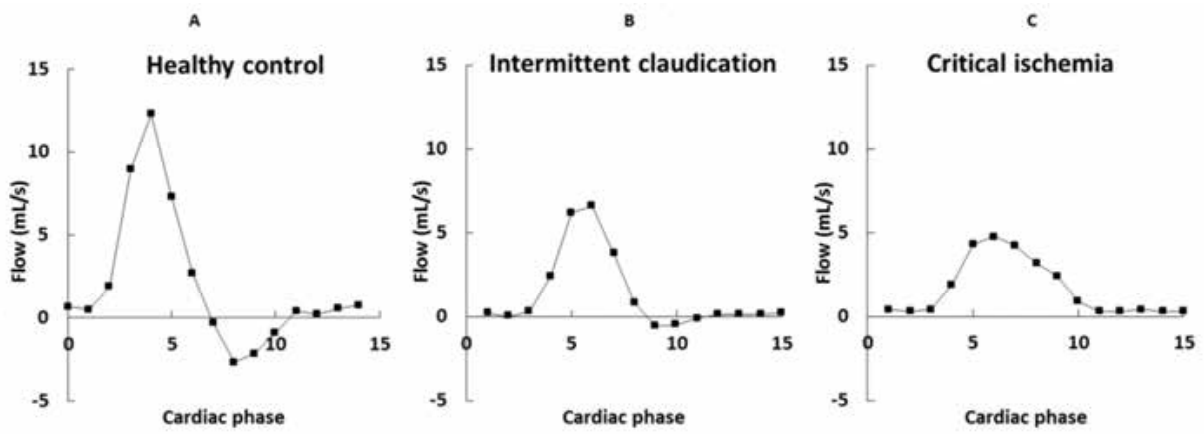

Figure 1. Flow waveforms in a healthy subject without peripheral arterial disease (A), a patient with intermittent claudication (B) and critical ischemia (C). Note the tri-phasic flow waveform in the healthy subject, the bi-phasic flow pattern in a patient with intermittent claudication and mono-phasic flow waveform in a patient with critical ischemia. The APF is lowest in the patient with critical ischemia and highest in the healthy control.

Typical flow waveforms obtained in a patient with intermittent claudication and critical ischemia are presented in figure 1. APF values in patients with PAD with and without relevant stenoses and non-PAD subjects are listed in table 2 . Average APF values in PAD patients with relevant stenoses (mean $\pm \mathrm{SD}, 4.6 \pm 3.0 \mathrm{~mL} / \mathrm{s}$ ) were $58 \%$ lower compared to non-PAD subjects (i.e. non-PAD patients and healthy subjects) $(11.0 \pm 3.5 \mathrm{~mL} / \mathrm{s}, \mathrm{p}<0.01)$. APF was significantly lower in PAD patients with relevant stenosis compared to PAD patients without relevant stenoses $(p<0.01)$. In PAD patients without relevant stenosis APF was $21 \%$ lower compared to non-PAD subjects $(p=0.01)$. Within the group of non-PAD subjects, no difference was found in absolute APF values between non-PAD patients $(11.3 \pm 3.8 \mathrm{~mL} / \mathrm{s})$ and healthy subjects $(10.5$ $\pm 3.1 \mathrm{~mL} / \mathrm{s}, \mathrm{p}=0.35$ ). Relevant stenoses were present in the lower leg 
in only 14 PAD patients. In these patients, APF was $7.6 \pm 4.4 \mathrm{~mL} / \mathrm{s}$, which was significantly lower compared to non-PAD subjects $(p<0.01)$.

Average APF values in different subgroups of PAD patients are listed in table 3. APF was significantly lower in PAD patients with critical ischemia $(4.1 \pm 3.2 \mathrm{~mL} / \mathrm{s})$ compared those with intermittent claudication $(5.1 \pm 2.9 \mathrm{~mL} / \mathrm{s}, \mathrm{p}=0.02)$. The difference in average APF values between PAD patients with diabetes mellitus and without diabetes mellitus was not statistically significant $(p=0.13)$. However, the subgroup of PAD patients with critical ischemia and diabetes mellitus had a significantly higher APF $(4.9 \pm 3.9 \mathrm{~mL} / \mathrm{s})$ compared to the non-diabetic patients with critical ischemia $(3.4 \pm 2.3 \mathrm{~mL} / \mathrm{s}, \mathrm{p}=0.04)$.

\begin{tabular}{ccccccc}
\hline & \multicolumn{2}{c}{ Intermittent claudication } & \multicolumn{2}{c}{ Critical ischemia } & \multicolumn{2}{c}{ Total } \\
Diabetes mellitus & APF $(\mathbf{m L} / \mathbf{s})$ & $\mathbf{n}$ & APF $(\mathbf{m L} / \mathbf{s})$ & $\mathbf{n}$ & APF $(\mathbf{m L} / \mathbf{s})$ & $\mathbf{n}$ \\
\hline Yes & $5.1 \pm 3.2$ & 48 & $4.9 \pm 3.9$ & 49 & $5.0 \pm 3.5$ & 97 \\
No & $5.0 \pm 2.8$ & 46 & $3.4 \pm 2.3$ & 42 & $4.3 \pm 2.7$ & 88 \\
Unknown & $5.0 \pm 2.2$ & 19 & $3.1 \pm 1.7$ & 14 & $4.2 \pm 2.2$ & 33 \\
Total & $5.1 \pm 2.9$ & 113 & $4.1 \pm 3.2$ & 105 & $4.6 \pm 3.0$ & 218 \\
\hline
\end{tabular}

Table 3. Arterial peak flow in subgroups of patients with PAD with relevant stenosis.

Values are presented as mean \pm SD. PAD, peripheral arterial disease; APF, arterial peak flow.

The distribution of APF values in the (most) symptomatic leg of PAD patients with relevant stenosis and the right legs of non-PAD subjects at the level of the PA is graphically presented in figure 2 . In general, APF values in PAD patients were lower compared to non-PAD subjects, although a certain degree of overlap was observed.

The ability of APF to discriminate between PAD patients and nonPAD subjects is visualized in the ROC curves shown in figure 3 . The area-under-the-curve (AUC) value for all PAD patients was 0.90 with a 95\% confidence interval $(\mathrm{Cl})$ between $0.86-0.95$. For PAD patients with intermittent claudication and critical ischemia, AUC's were $0.92(95 \% \mathrm{CI}$ $0.89-0.96)$ and $0.93(95 \% \mathrm{Cl} 0.90-0.97)$, respectively. The ability to 


\section{CHAPTER 5}

differentiate between PAD patients with intermittent claudication and critical ischemia revealed a AUC of $0.63(95 \% \mathrm{Cl} 0.56-0.71)$.

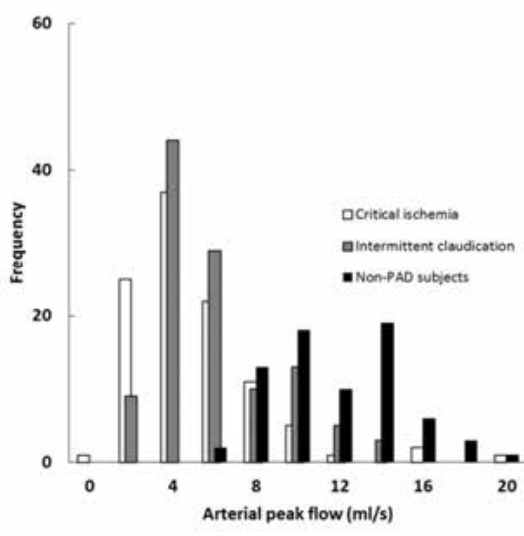

Figure 2. Histogram showing the distribution of arterial peak flow values at the level popliteal artery among a PAD patients with intermittent claudication and critical ischemia and a nonPAD subject population.

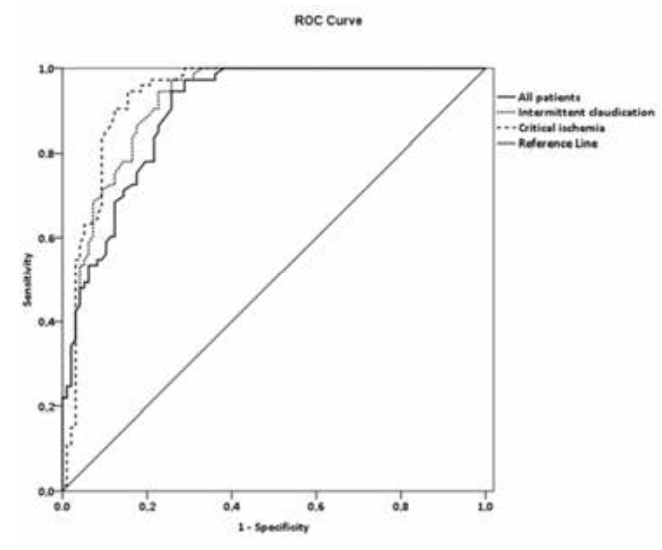

Figure 3. Receiver operating characteristic (ROC) curves showing ability of APF values to discriminate between PAD.

No correlation was found between APF and age $(R=0.0022)$ in the non-PAD subjects of this study.

\section{Discussion}

The presence of PAD results in decreased flow to the affected lower extremity. However, the exact impact of various degrees of PAD on absolute MR derived flow values is not known. For this reason we sought to establish reference values for resting APF in a large sample of patients with PAD as well as patients referred for MRA of the lower extremities for non-PAD indications, and a sample of healthy control subjects. To the best of our knowledge, this study is the first to report such data of APF values in patients with PAD. 
Literature about resting APF in the peripheral vascular tree in patients with PAD is sparse. Most studies providing MR derived APF used young, healthy subjects $(6,7)$, whereas others mainly focused on flow waveform analysis and/or blood velocity or mean flow rather than (peak) flow $(16,18)$. Other imaging techniques such as duplex ultrasonography also focus on blood velocity, but are mostly measured in and directly adjacent to stenotic areas (i.e. peak systolic velocity ratio) $(9,19)$. Therefore, no direct comparison of our results with other studies seems possible.

Although MRA is a highly reliable test to determine the location and severity of peripheral vascular lesions, it remains difficult to draw conclusions with regard to their hemodynamic significance based on anatomical information alone. In our opinion the APF provides relevant complementary functional information on vascular status that can measured in a fast, simple and reproducible way.

Here we demonstrate a standardized and simple approach in which APF was measured in the PA of patients with PAD (regardless of the location of arterial narrowing), and in non-PAD subjects. Mean APF values in PAD were significantly lower compared to non-PAD subjects. Mean APF was $58 \%$ lower in PAD patients with a clinical relevant stenosis compared to non-PAD subjects. A simple explanation for this finding is that PA arterial peak flow reflects - to a certain extent - all proximal obstructive arterial lesions.

The APF in PAD patients with critical ischemia was significantly lower compared to patients with intermittent claudication. This was expected, as critical ischemia is clinically the most severe form of PAD where perfusion under resting conditions is not able to meet metabolic needs. No difference was found in APF of PAD patients with and without diabetes mellitus. However, for patients with critical ischemia, APF was higher in the diabetic subpopulation. Higher flow values in these patients with severe PAD are possibly due to severely calcified arteries which are known to exhibit decreased vascular compliance, resulting in an impaired 'windkessel' function of the lower leg arteries (20). 


\section{CHAPTER 5}

As we showed in the population of non-PAD subjects, the APF is not correlated with age. This is advantageous, as this indicates that age is not likely to be a relevant confounding factor.

\section{Discriminative value}

The discriminative ability of APF measurements to differentiate patients with peripheral vascular stenoses exceeding $50 \%$ reduction in luminal diameter versus non-PAD subjects as expressed by the area under the ROC curves was excellent (figure 3). Although the AUC was largest for the distinction between PAD patients with critical ischemia and non-PAD subjects (0.93), PAD patients with intermittent claudication could also be distinguished with high accuracy from those not suffering from PAD as well as patients with lower degrees of stenosis (AUC, 0.92). The APF is less suitable to distinguish between patients with intermittent claudication and critical ischemia (AUC, 0.63).

\section{Clinical implications}

Objective assessment of the functional consequences of obstructive PAD is a necessity in diagnosis and longitudinal follow-up and/or therapy monitoring of these patients. We believe APF could be a useful adjunct to morphological evaluation of the peripheral vasculature with CE-MRA, especially in patients with intermediate degrees of stenosis and equivocal results at standard functional testing. Currently, the ankle-brachial index ( $A B I)$ measurement is the most recognized and most widely applied functional measurement used for diagnosis and therapy monitoring $(2,21,22)$. ABI measurements, however, are hampered by poor reproducibility and cannot be determined accurately in patients with heavily calcified arteries (23-25). Other known techniques to determine vascular function are Doppler-based flow measurements, plethysmography, skin perfusion pressure measurements, positron emission tomography (PET), contrast-enhanced ultrasound (CEUS) and intra-arterial pressure and/or flow measurements (26-35). These techniques, however, may lack spatial resolution or coverage, or are unfavorable for repeated measurements for instance in the context of 
therapy monitoring due to time constraints, invasiveness, use of ionizing radiation, or large interobserver variability (8).

\section{Study Limitations}

The most important limitation of this study is that as far as PAD patients were concerned, only patients scheduled for MRA were included. Although this resulted in a selected study population, this population was considered to be clinically the most relevant as far as APF measurements are concerned, as MRI-based APF measurements are not used for screening purposes due to the limited availability of MRI systems or magnet time and associated costs. Nevertheless, because the study sample comprised consecutive patients undergoing CE-MRA over a period of 1 year, it is a representative group with regard to the casemix one is likely to encounter in every day clinical practice. This also implies that current findings do not necessarily apply to all PAD patients. A second limitation is that although the group of non-PAD patients who were used as control subjects were free from PAD (according to both clinical history as well as findings at CE-MRA), they were not entirely free from cardiovascular disease, as the majority of the nonPAD patients had experienced prior deep venous thrombosis. Nevertheless, APF measures of these non-PAD patients were comparable with healthy controls from previous studies $(6,7)$. Finally, we did not measure APF in arteries below the knee. This was because limitations in spatial and temporal resolution in the acquisition protocol used in this study precluded sufficiently accurate APF measurements in these small arteries $(14,36,37)$. Yet, as our results have shown, APF measurements at the level of the PA are sensitive enough to detect arterial stenosis in the lower leg.

In conclusion, this study provides MRI derived reference values for resting APF in patients with clinically manifest PAD as well as a control sample of patients referred for non-PAD indications and healthy subjects. These reference values may aid in objective functional quantification of PAD severity and follow-up after intervention. We found that 


\section{CHAPTER 5}

absolute flow values allow diagnosis of clinically relevant PAD with high accuracy. 


\section{References}

1. Aboyans V, Ho E, Denenberg JO, et al. The association between elevated ankle systolic pressures and peripheral occlusive arterial disease in diabetic and nondiabetic subjects. J Vasc Surg. 2008;48(5):1197-203.

2. Norgren L, Hiatt WR, Dormandy JA, et al. Inter-Society Consensus for the Management of Peripheral Arterial Disease (TASC II). J Vasc Surg. 2007;45 Suppl S:S5-67.

3. de Vries M, de Koning PJ, de Haan MW, et al. Accuracy of semiautomated analysis of 3D contrast-enhanced magnetic resonance angiography for detection and quantification of aortoiliac stenoses. Invest Radiol. 2005;40(8):495-503.

4. de Vries M, Nijenhuis RJ, Hoogeveen RM, et al. Contrast-enhanced peripheral MR angiography using SENSE in multiple stations: feasibility study. J Magn Reson Imaging. 2005;21(1):37-45.

5. Leiner T, Kessels AG, Nelemans PJ, et al. Peripheral arterial disease: comparison of color duplex US and contrast-enhanced MR angiography for diagnosis. Radiology. 2005;235(2):699-708.

6. Versluis B, Backes WH, van Eupen MG, et al. Magnetic resonance imaging in peripheral arterial disease: reproducibility of the assessment of morphological and functional vascular status. Invest Radiol. 2011;46(1):1124.

7. Versluis B, Dremmen MH, Nelemans PJ, et al. MRI of arterial flow reserve in patients with intermittent claudication: feasibility and initial experience. PLoS One. 2012;7(3):e31514.

8. van Langen $\mathrm{H}$, van Gurp J,Rubbens $\mathrm{L}$. Interobserver variability of anklebrachial index measurements at rest and post exercise in patients with intermittent claudication. Vasc Med. 2009;14(3):221-6.

9. Begelman SM, Jaff MR. Noninvasive diagnostic strategies for peripheral arterial disease. Cleve Clin J Med. 2006;73 Suppl 4:S22-9.

10. Corcoran HA, Smith BE, Mathers $\mathrm{P}$, et al. Laser Doppler imaging of reactive hyperemia exposes blood flow deficits in a rat model of experimental limb ischemia. J Cardiovasc Pharmacol. 2009;53(6):446-51.

11. Kido M, Takeuchi S, Hayashida S, et al. Assessment of abnormal blood flow and efficacy of treatment in patients with systemic sclerosis using a newly developed microwireless laser Doppler flowmeter and arm-raising test. Br J Dermatol. 2007;1 57(4):690-7. 


\section{CHAPTER 5}

12. Dumoulin CL. Phase contrast MR angiography techniques. Magn Reson Imaging Clin N Am. 1995;3(3):399-411.

13. Walker MF, Souza SP, Dumoulin CL. Quantitative flow measurement in phase contrast MR angiography. J Comput Assist Tomogr. 1988;12(2):304-13.

14. Bakker CJ, Hoogeveen RM, Viergever MA. Construction of a protocol for measuring blood flow by two-dimensional phase-contrast MRA. J Magn Reson Imaging. 1999;9(1):119-27.

15. Leiner T, Nijenhuis RJ, Maki JH, et al. Use of a three-station phased array coil to improve peripheral contrast-enhanced magnetic resonance angiography. J Magn Reson Imaging. 2004;20(3):417-25.

16. Mohajer K, Zhang H, Gurell D, et al. Superficial femoral artery occlusive disease severity correlates with MR cine phase-contrast flow measurements. J Magn Reson Imaging. 2006;23(3):355-60.

17. Prakash A, Garg R, Marcus EN, et al. Faster flow quantification using sensitivity encoding for velocity-encoded cine magnetic resonance imaging: in vitro and in vivo validation. J Magn Reson Imaging. 2006;24(3):676-82.

18. Klein WM, Bartels LW, Bax L, et al. Magnetic resonance imaging measurement of blood volume flow in peripheral arteries in healthy subjects. J Vasc Surg. 2003;38(5):1060-6.

19. Owen AR, Roditi GH. Peripheral arterial disease: the evolving role of noninvasive imaging. Postgrad Med J. 201 1;87(1025):189-98.

20. Tozeren A. Elastic properties of arteries and their influence on the cardiovascular system. J Biomech Eng. 1984;106(2):182-5.

21. Hirsch AT, Haskal ZJ, Hertzer NR, et al. ACC/AHA 2005 Practice Guidelines for the management of patients with peripheral arterial disease (lower extremity, renal, mesenteric, and abdominal aortic): a collaborative report from the American Association for Vascular Surgery/Society for Vascular Surgery, Society for Cardiovascular Angiography and Interventions, Society for Vascular Medicine and Biology, Society of Interventional Radiology, and the ACC/AHA Task Force on Practice Guidelines (Writing Committee to Develop Guidelines for the Management of Patients With Peripheral Arterial Disease): endorsed by the American Association of Cardiovascular and Pulmonary Rehabilitation; National Heart, Lung, and Blood Institute; Society for Vascular Nursing; TransAtlantic Inter-Society Consensus; and Vascular Disease Foundation. Circulation. 2006;113(11):e463-654. 
22. Creager MA. Clinical assessment of the patient with claudication: the role of the vascular laboratory. Vasc Med. 1997;2(3):231-7.

23. Lindner JR, Womack L, Barrett EJ, et al. Limb stress-rest perfusion imaging with contrast ultrasound for the assessment of peripheral arterial disease severity. JACC Cardiovasc Imaging. 2008;1 (3):343-50.

24. Stein R, Hriljac I, Halperin JL, et al. Limitation of the resting ankle-brachial index in symptomatic patients with peripheral arterial disease. Vasc Med. 2006;11(1):29-33.

25. Al-Qaisi $M$, Nott $D M$, King $D H$, et al. Ankle brachial pressure index (ABPI): An update for practitioners. Vasc Health Risk Manag. 2009;5:833-41.

26. Wu WC, Wang J, Detre JA, et al. Hyperemic flow heterogeneity within the calf, foot, and forearm measured with continuous arterial spin labeling MRI. Am J Physiol Heart Circ Physiol. 2008;294(5):H2129-36.

27. Burchert W, Schellong S, van den Hoff J, et al. Oxygen-15-water PET assessment of muscular blood flow in peripheral vascular disease. J Nucl Med. 1997;38(1):93-8.

28. Bragadeesh T, Sari I, Pascotto M, et al. Detection of peripheral vascular stenosis by assessing skeletal muscle flow reserve. J Am Coll Cardiol. 2005;45(5):780-5.

29. Peetrons P. Ultrasound of muscles. Eur Radiol. 2002;12(1):35-43.

30. Chloros GD, Smerlis NN, Li Z, et al. Noninvasive evaluation of upperextremity vascular perfusion. J Hand Surg Am. 2008;33(4):591-600.

31. Weber MA, Krix M, Delorme S. Quantitative evaluation of muscle perfusion with CEUS and with MR. Eur Radiol. 2007;17(10):2663-74.

32. Ludman PF, Volterrani M, Clark AL, et al. Skeletal muscle blood flow in heart failure measured by ultrafast computed tomography: validation by comparison with plethysmography. Cardiovasc Res. 1993;27(6):1109-15.

33. Duet M, Virally M, Bailliart O, et al. Whole-body (201)TI scintigraphy can detect exercise lower limb perfusion abnormalities in asymptomatic diabetic patients with normal Doppler pressure indices. Nucl Med Commun. 2001;22(9):949-54.

34. Ament W, Lubbers J, Rakhorst G, et al. Skeletal muscle perfusion measured by positron emission tomography during exercise. Pflugers Arch. 1998;436(5):653-8.

35. Fleischer AC, Niermann KJ, Donnelly EF, et al. Sonographic depiction of microvessel perfusion: principles and potential. J Ultrasound Med. 2004;23(11):1499-506. 


\section{CHAPTER 5}

36. Hoogeveen RM, Bakker CJ, Viergever MA. MR phase-contrast flow measurement with limited spatial resolution in small vessels: value of modelbased image analysis. Magn Reson Med. 1999;41(3):520-8.

37. Machida $\mathrm{H}$, Komori $\mathrm{Y}$, Ueno $\mathrm{E}$, et al. Accurate measurement of pulsatile flow velocity in a small tube phantom: comparison of phase-contrast cine magnetic resonance imaging and intraluminal Doppler guidewire. Jpn J Radiol. 2010;28(8):571-7. 


\section{CHAPTER 6}

\section{Magnetic resonance imaging-based monitoring}

of collateral artery development in patients with

intermittent claudication during supervised

exercise therapy

B. Versluis, T. Leiner, P.J. Nelemans, J.E. Wildberger, G.W.H. Schurink, W.H. Backes

Published in the Journal of Vascular Surgery, 2013:

Vers/uis B, Leiner T, Nelemans $P$ J, et al. Magnetic resonance imagingbased monitoring of collateral artery development in patients with intermitten claudication during supervised exercise therapy. J Vasc Surg. 2013 


\section{CHAPTER 6}

\section{Abstract}

\section{Objectives}

The purpose of the current study was to determine whether supervised exercise therapy (SET) leads to measurable vascular adaptations in patients with intermittent claudication using contrast-enhanced MR angiography (CE-MRA) and flow measurements.

\section{Materials and Methods}

Ten patients with clinical symptoms of intermittent claudication and proven obstructive arterial lesions of the superficial femoral artery were included and underwent SET for a period of six months. At baseline, all patients underwent a treadmill test to assess pain free walking distance, ankle-brachial pressure index (ABI) measurement and MR imaging to determine the number of arteries in the upper leg using CEMRA and arterial peak flow (APF) in the popliteal artery using cine phase contrast angiography. All examinations were repeated after three and six months of SET, respectively, to evaluate vascular responses relative to baseline.

\section{Results}

After six months of SET, mean pain free walking distance (395 \pm $46 \mathrm{~m}$ ) was improved by $71 \%$ compared to baseline $(230 \pm 42 \mathrm{~m}, \mathrm{p}<$ $0.01)$. No statistically significant changes were found for mean values of $\mathrm{ABI}(81 \pm 4$ and $77 \pm 4$ at respectively baseline and after six months of SET), number of arteries at the level of the arterial lesion (18 \pm 2 and 19 \pm 2 , respectively) nor arterial peak flow $(5.3 \pm 0.6$ and $5.3 \pm 0.8 \mathrm{~mL} / \mathrm{s}$, respectively). 


\section{Conclusions}

Supervised exercise therapy in patients with intermittent claudication results in an increase in pain free walking distance, whereas with MRI no macrovascular adaptations in terms of additional collateral artery formation or flow increases could be observed. 


\section{Introduction}

Peripheral arterial disease (PAD) is a major health care problem among the elderly in the Western society $(1,2)$, affecting up to $7 \%$ of the general population over the age of 70 years (2). Symptoms in these patients range from intermittent claudication (Fontaine stage II, Rutherford stage 1-3 (3)) to critical limb ischemia (1).

The primary aim of treatment, in symptomatic patients with PAD without signs of critical ischemia, is relief of symptoms by improving exercise performance. Structured or supervised exercise therapy (SET) has been shown to be an effective conservative therapeutic approach to achieve this aim (2, 4-7). Improvement in exercise performance with SET is well established (5-8), but the magnitude of the response to SET varies according to literature.

Although SET is the primary choice of treatment in intermittent claudication, the exact physiological mechanisms by which clinical improvement is accomplished are still subject of debate (6). Stimulating collateral artery formation to increase muscle perfusion is thought to be a potentially contributing biological mechanism $(2,6,7,9-12)$, which is generally thought to be a response to exercise induced elevated vessel wall shear stress $(13,14)$. Collateral artery formation is a complicated process and is thought to involve both adaptation of existing arteries and the formation of novel collateral arteries over time (15). To date the exact conditions under which collateral formation take place in patients with PAD, as well determinants of variations in extent of collateral formation among patients are still poorly understood.

Magnetic resonance imaging is an ideal modality to study the process of vascular adaptations in the context of SET because it is highly reliable for depiction of the peripheral vascular tree (16-18) and the ability to quantitatively study physiological phenomena such as vascular flow.

The purpose of this study was to assess the morphological and functional macrovascular adaptations in response to SET in patients with 
intermittent claudication. Morphological effects were evaluated with contrast-enhanced MR angiography (CE-MRA), whereas functional effects were assessed using quantitative flow measurements.

\section{Materials and Methods}

\section{Study population}

Patients with newly diagnosed symptoms of PAD (Fontaine stage II; intermittent claudication with no signs of critical ischemia (3)) were eligible for participation in this study if obstructive arterial lesions (i.e. significant stenosis (> 50\%) and/or occlusion) of the superficial femoral artery (SFA) were detected by duplex ultrasonography (peak systolic velocity (PSV) ratio $>2.0$ (19)). Focus in this study was on patients with SFA disease as these patients are known to respond best to SET, whereas patients with obstructive lesions within the iliac arteries are more often referred for invasive therapy and more likely to drop out of SET. Patients with lower leg stenosis are more prone to have diabetes mellitus, in which collateral artery formation might be impaired (20-22). Therefore, diabetic patients and patients with significant arterial lesions in the aorta, iliac arteries or arteries below the knee were excluded. If significant arterial lesions were detected in either the infra-renal aorta or severe lesions were detected in the lower leg on CE-MRA after initial inclusion of a patient, they were excluded from further participation from this study, however, this scenario did not occur during the entire study. Additional exclusion criteria were: prior treatment for PAD and presence of chronic or acute critical ischemia. Patients scheduled for vascular surgery or percutaneous transluminal angioplasty were also excluded from participation, as well as patients with contra-indications to MRI, including claustrophobia, known gadolinium based contrast agent allergy, and low estimated glomerular filtration rate $(<30$ $\mathrm{mL} / \mathrm{kg} / 1.73 \mathrm{~m}^{2}$ ) or patients with contra-indications for SET. The study was approved by the institutional medical ethics committee and all patients gave written informed consent before inclusion. 


\section{Study design}

Upon entry in the study, all patients underwent a treadmill test, ankle-brachial pressure index (ABI) measurement and MRI as described below to establish baseline values. MRI was acquired first, followed by the $A B I$ measurements and the treadmill test on the same day (figure 1). All exams were repeated after three and six months of SET, respectively. Patients did not receive other (invasive) therapy than SET during this period, nor were there any alterations in prescribed medicaments. After six months of SET, patients returned to their vascular surgeon for evaluation and to discuss further treatment options (i.e. (dis)continuing SET and/or invasive therapy). Participation in this study had no impact on the course of treatment for the patients. MRI data were not used in the clinical evaluation of the participants.

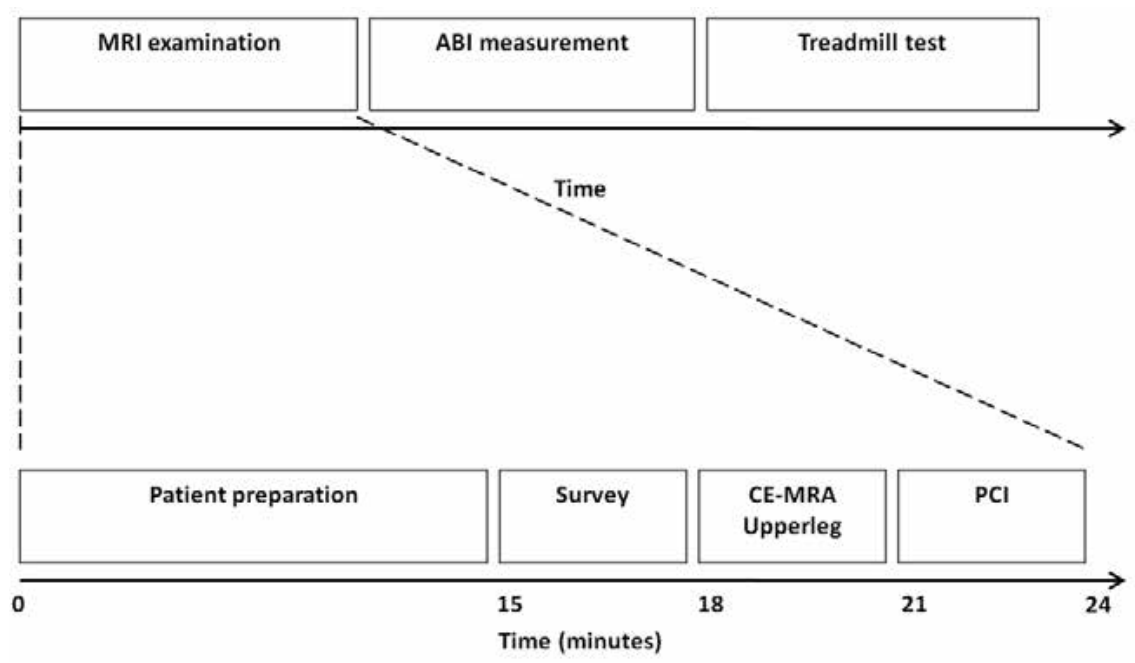

Figure 1. Overview of the imaging protocol. The MRI examination was performed first, followed by the $A B I$ measurement and treadmill test for each visit of the patient. During the MRI examination, a first-pass contrast-enhanced MR angiography (CE-MRA) was performed of the entire muscular volume of the upper leg. CE-MRA was followed by quantitative cine phase-contrast imaging $(\mathrm{PCl})$ to measure the arterial peak flow. 


\section{SET and treadmill testing}

A standardized and graded treadmill protocol was used during both the treadmill tests as well as the subsequent SET, as previously described by Gardner et al. (23). In this widely accepted and validated protocol (23), a constant speed of $3.2 \mathrm{~km} / \mathrm{h}$ was applied, whereas during the exercise the slope of the treadmill was increased by $2 \%$ every two minutes starting at $0 \%$ inclination, thereby gradually increasing the metabolic demand for the patient. SET consisted of walking exercises on a treadmill 2-3 times per week, supervised by a physiotherapist. In addition, patients were given instructions for individual walking exercises.

To assess the effect of SET on pain free walking distance, treadmill tests were performed after three and six months at the vascular laboratory of our institution.

\section{MRI protocol}

MRI was performed to determine morphological and functional vascular effects during SET. Morphologic effects were studied using contrast-enhanced MR angiography (CE-MRA), whereas functional effects were studied using quantitative $2 D$ cine MR phase-contrast imaging ( $\mathrm{PCl}$ ) to determine the arterial peak flow (APF) of the popliteal artery both at rest and after provoking reactive hyperemia to determine the maximum hyperemic flow as described before $(24,25)$. Postischemic reactive hyperemia in the lower leg was provoked using an inflatable cuff, ensuring total arterial occlusion (Medrad, indianola, PA). The cuff was placed at mid-thigh level and manually inflated to suprasystolic values (> $50 \mathrm{mmHg}$ above brachial systolic blood pressure) during 6 minutes. During cuff inflation, flow in the PA was measured with cine $\mathrm{PCl}$ to check that total arterial occlusion of the upper leg was achieved. A schematic overview of the study and MRI measurements is given in figure 1 .

All examinations were performed on a 1.5-T MRI system (Intera, Philips Medical Systems, Best, The Netherlands). For signal reception we 


\section{CHAPTER 6}

used a dedicated 12-element phased-array peripheral vascular coil with a craniocaudal coverage of $128 \mathrm{~cm}$ (Philips Medical Systems). Patients were imaged in supine position and were in this position for approximately 30 minutes before the flow measurement was started to minimize any confounding history of physical effort. During this time the CE-MRA was acquired, using a fixed dose of $10 \mathrm{~mL}$ gadofosveset trisodium (Ablavar ${ }^{\circ}$, Lantheus Medical Imaging, Billerica, MA) as contrast agent.

Survey. A non-enhanced time-of-flight (TOF) scan of the pelvic, upper and lower leg station was acquired to prescribe the imaging volumes of interest for morphological and functional imaging. For this, a turbo field echo (TFE) pulse sequence was used with a $180^{\circ}$ inversion prepulse to suppress stationary tissues. Thirty-one axial slices per station were acquired with 3.3- $\mathrm{mm}$ slice thickness and 11-mm interslice gap, and an inferiorly concatenated saturation band. The standard quadrature body coil was used for signal transmission and reception. For positioning of the 3D CE-MRA volumes maximum intensity projections (MIP) were generated in 3 orthogonal directions.

Angiography. A one-station 3D gradient-echo sequence was used for first-pass CE-MRA as previously described $(16,26)$. Whereas clinical CE-MRA examinations are usually limited to the visualization of a thin anteroposterior imaging volume only including the large conduit arteries in the upper leg (i.e. SFA and PA), for this study the entire muscular volume of the upper leg was imaged to visualize all the arteries including any collateral branches within the upper leg. Acquisition parameters were as follows: TR $4.8 \mathrm{~ms}$, TE $1.45 \mathrm{~ms}$, flip angle $40^{\circ}$, FOV $470 \mathrm{~mm}$, matrix $480 \times 480$, and voxel dimensions (reconstructed) $0.92 \times 0.92 \times$ $1.20 \mathrm{~mm}$. Prior to contrast medium administration, a non-enhanced 'mask' image data set was acquired with exactly the same acquisition parameters as the CE-MRA, enabling background tissue suppression by image subtraction.

After the first-pass sequence, a three station non-subtracted high-resolution steady-state acquisition was applied to visualize the 
entire peripheral vascular tree to exclude iliac and lower leg arterial obstructions.

Flow MRI. For quantitative flow measurements we used a 2D gradient-echo cine phase contrast angiographic scan technique with the following acquisition parameters: TR $9.7 \mathrm{~ms}$, TE $5.8 \mathrm{~ms}$, flip angle $30^{\circ}$, FOV $380 \mathrm{~mm}$, matrix 384, and reconstructed voxel dimensions of $0.99 \mathrm{x}$ $0.99 \times 6.00 \mathrm{~mm}$. Fifteen dynamic phases were acquired to obtain flow waveforms over the cardiac cycle. The phase encoding velocity (VENC) was set to $100 \mathrm{~cm} / \mathrm{s}$ in the craniocaudal direction $(17,24)$. Vector cardiography triggering was used for retrospective cardiac synchronization. Parallel imaging (sensitivity encoding, SENSE) was applied to reduce scan time (SENSE acceleration factor 2 in the anterior-posterior direction) (27). At a mean heart rate of 60 beats per minute, the nominal acquisition time was 1 minute.

\section{$A B I$ measurements}

All $A B I$ measurements were performed by well-trained operators at our vascular function laboratory, using an automated Doppler system (Nicolet VasoGuard, VIASYS Healthcare, Madison, WI). If the ABI could not be determined due to arterial stiffness or other circumstances, this was mentioned in the report by the operators.

\section{Angiographic reading}

All angiographic data sets were analyzed by a well-trained radiologist with over 5 years of experience in CE-MRA of the peripheral vasculature. First-pass CE-MRA data sets were used to assess morphologic vascular effects following SET.

Image analysis of the first-pass acquisition to determine the number of arteries of the upper leg was performed on a dedicated 


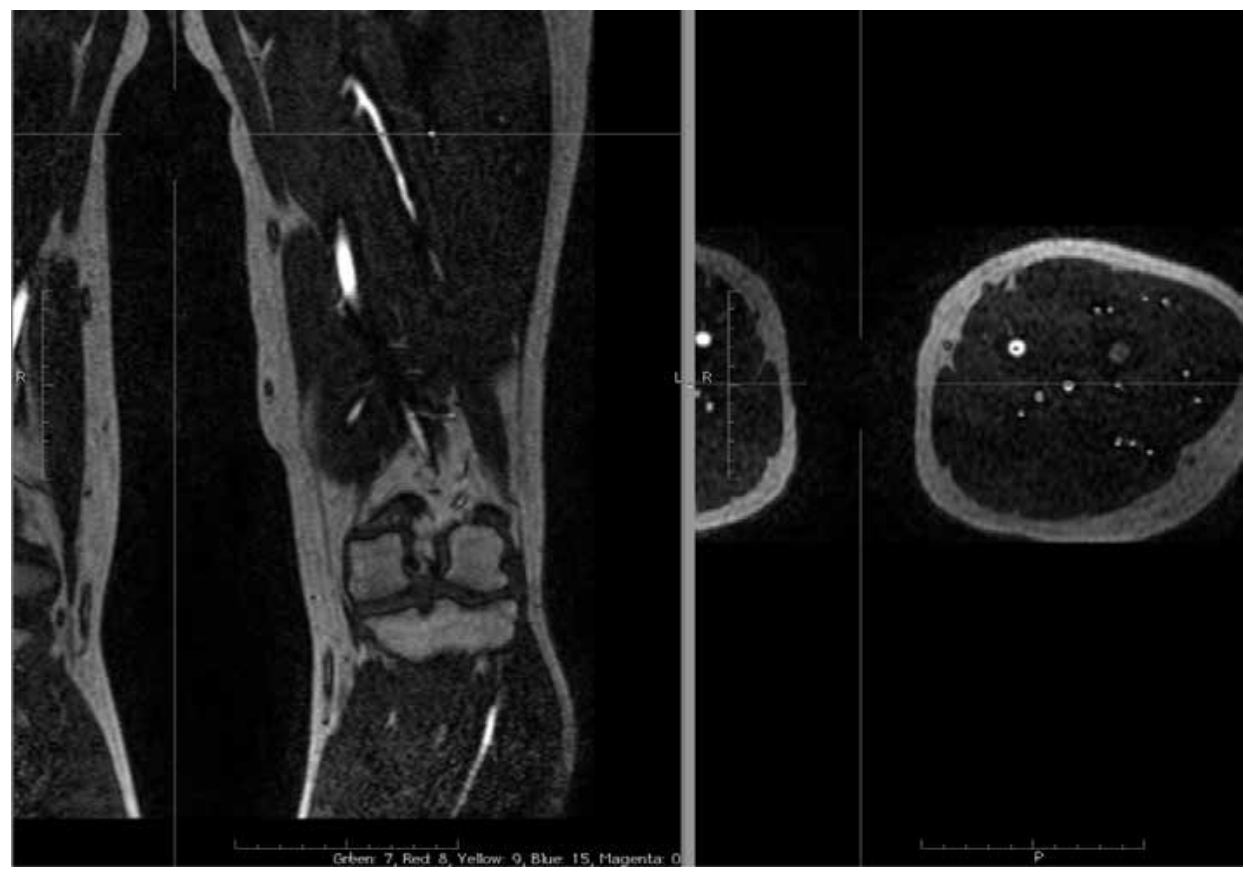

Figure 2. Example of the counting procedure of the arteries in the upper leg using firstpass CE-MRA data set. Arteries are marked in the right panel by the gray dots. 15 arteries were counted in this axial view.

image post processing workstation (MacPro, running OS $\mathrm{X}$, version 10.5.4, Apple Inc., Cupertino, CA). As contrast-enhanced source images have shown better reproducibility compared to subtracted images, source images were considered for analysis (17). The number of arteries was manually quantified by counting the total number of arteries crossing transverse planes in the upper leg at three different levels: $5 \mathrm{~cm}$ above, exactly at and $5 \mathrm{~cm}$ below the location where the SFA was reconstituted distal to the occlusion (figure 2) (17). This method ensures complete coverage of the collateral re-entrance zone, which represents the level at which collaterals re-enter the large native conduit artery distal to an occluded segment of the arterial tree (distal superficial femoral or popliteal artery in our study). Arteries were counted using the open source DICOM viewer OsiriX (OsiriX, Geneva, Switzerland, version 3.5.1, http://www.osirix-viewer.com/), using the original source images as well as coronal and sagittal multiplanar reconstructions (MPR). Identi- 
fication of the correct counting plane was ensured by cross-reference to the anatomical landmarks as described above. Subsequently, the total number of arteries intersecting the three planes was determined, including conduit arteries (SFA or PA), terminal muscle branches and any visible collateral arteries (figure 2). Arteries were distinguished from veins by scrolling through source images and MPR stacks to find an upstream connection to a named artery.

\section{Flow analysis}

Modulus and phase images were reconstructed from the cine phase contrast data. A quantitative flow analysis package included with the software release (Qflow, R1 1.4.14, Philips Medical Systems) of the MRI hardware was used for analysis of the flow waveform data. Using this software, a region of interest (ROI) covering the entire visible crosssection of the artery of interest was manually drawn using a reconstructed modulus image during peak systole and then automatically propagated to the remaining cardiac phases using an active contour algorithm. Although the peak systolic phase is sufficient to calculate the APF, ROls were propagated to all cardiac phases to obtain flow wave forms, which were visually analyzed to reassure that the chosen cardiac phase indeed was at peak systole and to detect possible aliasing effects directly after the acquisition. If detected, the measurement was repeated with sufficient higher phase encoding velocity. APF was preferred over mean flow for analysis, as peak flow previously proved much more reproducible and revealed large differences between patients and healthy controls $(17,24,25)$. Given the good inter-reader reproducibility of the APF analysis, all flow measurements were anonymously analyzed by a single, experienced radiologist $(17,25)$.

\section{Statistical analysis}

For statistical analysis only data of the symptomatic leg was used. If both legs were equally symptomatic, only the right leg was analyzed. Statistical analysis was performed with commercially available statistical software (SPSS 16.0, SPSS Inc., Chicago, IL). Mean values derived from 


\section{CHAPTER 6}

baseline and follow-up measurements were compared and tested for statistical significance using a $t$-test for paired samples. $\mathrm{P}<0.05$ was considered statistically significant.

\section{Results}

\section{Subjects}

Over a period of 6 months, 10 patients (mean age $72.8 \pm 8.9$ years; range 60 to 84 years; 8 men and 2 women) were included. SET was well tolerated by all patients. Subjects underwent six months of SET and three MRI examinations as planned without experiencing side effects or adverse events.

\section{Effect of SET on pain free walking distance}

Mean baseline pain free walking distance, as well as pain free walking distances after three and six months of SET are listed in table 1. After three months of SET the average pain free walking distance was significantly higher $(p<0.01)$ compared to the baseline distance at group level. After six months SET, pain free walking distance improved by $71 \%$ on average compared to baseline values $(p<0.01)$. The increase at six month relative to three month was small and not significant ( $\mathrm{p}=$ $0.45)$. In 2 out of 10 patients, pain free walking distance did not increase.

\section{MRA}

At baseline, a significant arterial stenosis $(n=6)$ or occlusion $(n=$ 4) was present in the SFA in all patients. Morphologically, the arterial lesions of most patients remained unchanged (figure 3), although in two patients there was evident progression of the arterial lesions after six months (figure 4), whereas clinical symptoms (i.e. pain free walking distance) in these patients remained unchanged. Collateral arteries were present in 6 out of 10 patients at baseline. The mean number of arteries 
in the upper leg at three different levels before and after SET is listed in table 1. There was no change in the mean number of collateral arteries after three and six months of SET, respectively, when compared to baseline values. In one of the two patients in whom the pain free walking distance did not improve, collateral arteries were present at baseline, while in the other no collateral arteries were present at baseline nor developed during SET.
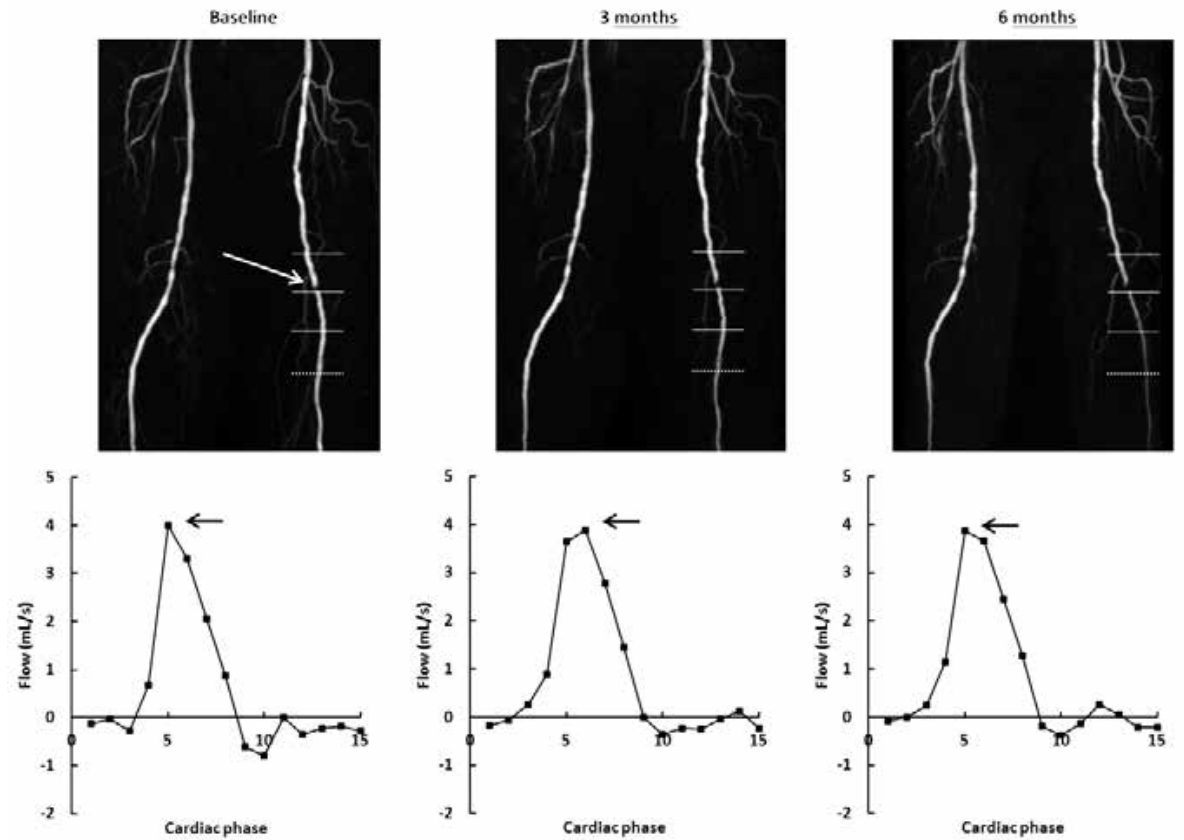

Figure 3. Example of a maximum intensity projection of CE-MRA (upper panels) and the arterial flow wave form (lower panels) of a responder to SET (pain free walking distance increased from $100 \mathrm{~m}$ to $333 \mathrm{~m}$ after six months of SET). The arterial obstruction in the SFA is indicated by the white arrow. The three white lines demarcate the three axial planes where arteries were counted. White dotted line represents the location of the APF measurement. No vascular morphologic or arterial peak flow (black arrows) changes were seen after six months of SET. 


\section{CHAPTER 6}

\section{APF}

Results of APF measurements are also listed in table 1. As was the case with the number of collateral arteries, mean APF at rest did not change significantly from the baseline value after three and six months of SET. Figure 3 shows an example of the APF at the level of the popliteal artery before and after SET in a patient that responded well to SET. Measurement of the maximum hyperemic flow after provoking reactive hyperemia with a cuff paradigm succeeded in 6 patients, while in 4 patients the cuff paradigm proved too painful and cuff inflation could not be maintained for 6 minutes. In those 6 patients with successful hyperemic measurements, the maximum hyperemic flow did not change significantly from baseline values either (table 1).

\begin{tabular}{lccc}
\hline & Baseline & 3 months & 6 months \\
\hline $\begin{array}{l}\text { Supervised Exercise Therapy } \\
\text { Pain free walking distance (m) }\end{array}$ & $230 \pm 42$ & $362 \pm 53^{a}$ & $395 \pm 46^{a}$ \\
$\begin{array}{l}\text { Number of arteries } \\
5 \mathrm{~cm} \text { above lesion }\end{array}$ & $23 \pm 3$ & $21 \pm 3$ & $20 \pm 3$ \\
At lesion level & $18 \pm 2$ & $18 \pm 3$ & $19 \pm 2$ \\
$5 \mathrm{~cm}$ below lesion & $12 \pm 1$ & $12 \pm 1$ & $12 \pm 1$ \\
Arterial Peak Flow (mL/s) & $5.3 \pm 0.6$ & $5.5 \pm 0.8$ & $5.3 \pm 0.8$ \\
Ankle-brachial index & $81 \pm 4$ & $80 \pm 4$ & $77 \pm 4$ \\
\hline
\end{tabular}

Table 1. Baseline and follow-up results after three and six months of SET, respectively. Values are presented as mean \pm SD. ap $<0.01$ compared to baseline.

\section{$\mathrm{ABI}$ measurements}

$A B I$ measures before and after three and six months of SET are listed in table 1 . In two patients, the ABI could not be determined reliably, due to uncompressible arteries at the level of the ankle. After three and six months of SET, the mean $A B I$ did not differ significantly from the baseline value. 


\section{Discussion}

In this study we assessed the macrovascular effects of six months SET in patients with intermittent claudication using different MRI techniques. The main finding of this study is that although pain free walking distance improved substantially and significantly, no vascular adaptations in terms of development of newly visible collateral arteries and/or increase in arterial peak flow could be detected with MRI.

\section{Effect of SET on walking distance}

Many studies have shown the benefits of supervised exercise therapy to increase pain free and maximum walking distance in patients with intermittent claudication $(2,4-8,28-31)$. Our study corroborates prior work and provides further evidence in support of this highly effective and low-cost intervention. The strongest improvement was made during the first three months of therapy, during which the pain free walking distance increased significantly compared to baseline values. Between the third and sixth month there was a trend of further improvement in walking performance, but improvements were less pronounced.

\section{SET and collateral artery development}

No new collateral arteries were found in our study population when comparing both maximum intensity projections as well as artery counts using our standardized counting method at different time points. On the contrary, in two patients arterial lesions increased in severity over time (figure 4).

Maximum intensity projections of CE-MRA data acquired at baseline revealed that most patients already had collateral arteries before the start of SET. The aspect of these collateral arteries did not change on MRA after six months of SET. Conversely, patients without collaterals at the start of SET did not develop any new visible collaterals during SET. These findings are remarkable, as SET is thought to stimulate formation 
of collateral arteries (6). Two patients in whom the pain free walking distance did not improve after SET, showed presence of collateral arteries at the start of SET already.

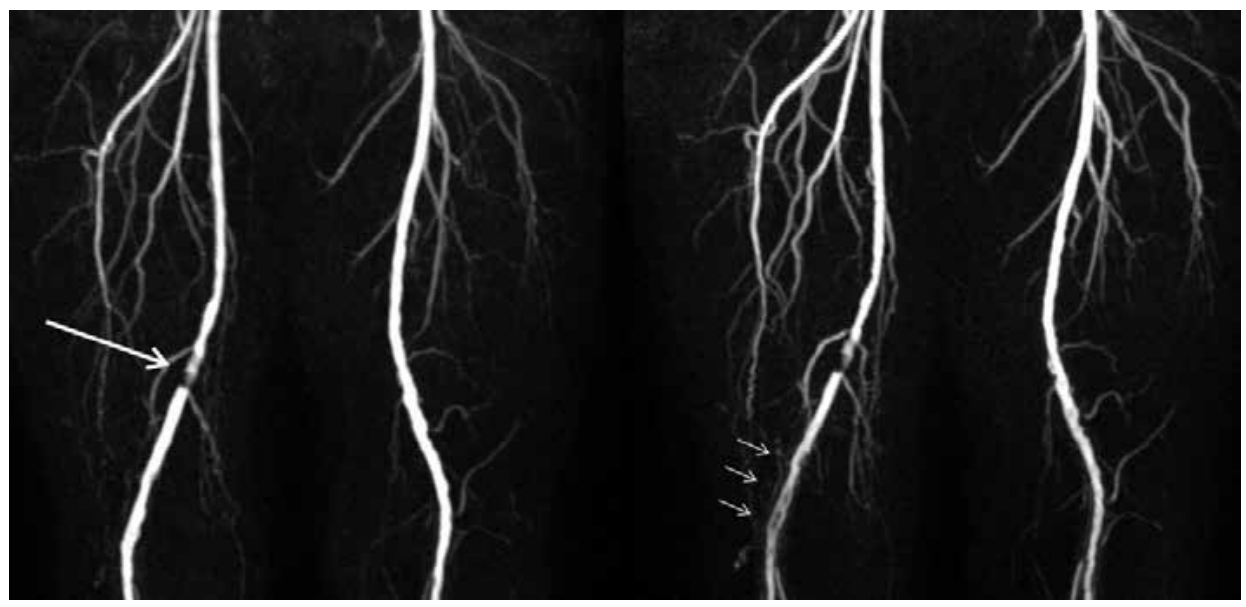

Figure 4. Progression of arterial lesions (white arrow left panel) in the right leg of a patient with intermittent claudication. At the level of the popliteal artery a new stenosis has developed (right panel, small white arrows) as compared to the baseline MRA (left panel).

APF

As with MRA, the APF measured at the level of the popliteal artery, i.e. below the level of the arterial stenosis in the superficial femoral artery and below the level of the suspected collateral artery re-entrance zone, did not reveal any improvement after three nor six months of SET, both at rest and after provoking reactive hyperemia (maximum hyperemic flow (25)). Even though there were no morphologic vascular adaptations, an increase in APF might still have been expected due to a possibly improved physical condition, especially were the hyperemic flow is concerned, yet APF results were in line with MRA findings. This means that clinical improvement of our patients cannot be explained by either morphologic or functional vascular changes, as far as could be detected with MRI at rest and after provoking hyperemia. 


\section{$A B I$}

The $A B I$ was measured to correlate possible functional vascular improvements (i.e. increased arterial peak flow) with a well-established and clinically widely applied functional test. Results in this study show that although the pain free walking distance of patients did increase, there was no increase in $A B I$ after respectively three and six months of SET, which is in line Watson et al (30), who did not find changes in $A B I$ during SET either. These results are also comparable with MRI findings, where no vascular adaptations or collateral artery formation was detected either.

\section{Considerations}

Although the study population was relatively small, we found no signs of macrovascular adaptations with MRI whereas there was a significant improvement in pain free walking distance after six months of SET in a high proportion of the participants. It is unlikely we missed significant collateral artery formation or improvements in flow as CEMRA has a high sensitivity for detecting small arteries (17) and both CEMRA as well as phase-contrast flow imaging proved highly reproducible before $(17,25)$. Given the observation that collateral arteries were already present in the majority of the included patients, it is more likely that clinical symptoms in these patients came apparent after collateral arteries had already been developed to a maximum extend and started to fall short.

Most patients in this study responded favorably to SET and improved clinically. Nevertheless, the success of SET does not seem to be related to a (macro) vascular substrate. Many participating patients declared, that in their opinion the improved pain free walking distance was actually the result of 'a better handling' of pain. Although we did not investigate this aspect it could potentially explain the discrepancy between clinical and MRI findings in this study (32).

This study demonstrates that active monitoring of SET with MRI is not helpful to unveil any contribution of marcovascular adaptations and 


\section{CHAPTER 6}

MRI cannot help at the early detection of possible non-responders. From a basic research point of view, it might be helpful to acquire MRI data of PAD patients in earlier stages of disease, during which no collateral arteries are present yet.

Even though MRI in this study was not able to detect macrovascular changes, our technique might still be useful for the monitoring and follow-up of patients with PAD treated with other, new therapeutic options aiming to improve vascularization, such as with stem cells and endothelial growth factors (i.e. vascular endothelial growth factor (VEGF) and basic fibroblast growth factor (bFGF)), which are currently being developed for treatment of both intermittent claudication and critical ischemia (33-36).

Finally, our study underscores the need for MR methods to study adaptations at tissue level. However, although some authors have investigated skeletal muscle oxygenation and perfusion (37-39) questions remain about the reproducibility of these techniques, at least at a magnetic field strength of $1.5 \mathrm{~T}$ (17).

\section{Conclusion}

Supervised exercise therapy in patients with intermittent claudication results in an increase in pain free walking distance. Nevertheless, with the current approach MRI proved not able to detect macrovascular adaptations in terms of additional collateral artery formation or flow increases both at rest and during reactive hyperemia after six months of SET compared to baseline values. 


\section{References}

1. Hirsch AT, Haskal ZJ, Hertzer NR, et al. ACC/AHA 2005 guidelines for the management of patients with peripheral arterial disease (lower extremity, renal, mesenteric, and abdominal aortic): executive summary a collaborative report from the American Association for Vascular Surgery/Society for Vascular Surgery, Society for Cardiovascular Angiography and Interventions, Society for Vascular Medicine and Biology, Society of Interventional Radiology, and the ACC/AHA Task Force on Practice Guidelines (Writing Committee to Develop Guidelines for the Management of Patients With Peripheral Arterial Disease) endorsed by the American Association of Cardiovascular and Pulmonary Rehabilitation; National Heart, Lung, and Blood Institute; Society for Vascular Nursing; TransAtlantic Inter-Society Consensus; and Vascular Disease Foundation. J Am Coll Cardiol. 2006;47(6): 1239-312.

2. Norgren L, Hiatt WR, Dormandy JA, et al. Inter-Society Consensus for the Management of Peripheral Arterial Disease (TASC II). J Vasc Surg. 2007;45 Suppl S:S5-67.

3. Aslam F, Haque A, Foody J, et al. Peripheral arterial disease: current perspectives and new trends in management. South Med J. 2009;102(11):1141-9.

4. Hiatt WR, Wolfel EE, Meier $\mathrm{RH}$, et al. Superiority of treadmill walking exercise versus strength training for patients with peripheral arterial disease. Implications for the mechanism of the training response. Circulation. 1994;90(4):1866-74.

5. Gardner AW, Poehlman ET. Exercise rehabilitation programs for the treatment of claudication pain. A meta-analysis. JAMA. 1995;274(12):975-80.

6. Stewart KJ, Hiatt WR, Regensteiner JG, et al. Exercise training for claudication. N Engl J Med. 2002;347(24):1941-51.

7. Leng GC, Fowler B, Ernst E. Exercise for intermittent claudication. Cochrane Database Syst Rev. 2000(2):CD000990.

8. Ahimastos AA, Pappas EP, Buttner PG, et al. A meta-analysis of the outcome of endovascular and noninvasive therapies in the treatment of intermittent claudication. J Vasc Surg. 2011;54(5):1511-21. 


\section{CHAPTER 6}

9. Schlager O, Giurgea A, Schuhfried O, et al. Exercise training increases endothelial progenitor cells and decreases asymmetric dimethylarginine in peripheral arterial disease: a randomized controlled trial. Atherosclerosis. $2011 ; 217(1): 240-8$.

10. Gavin TP, Wagner PD. Effect of short-term exercise training on angiogenic growth factor gene responses in rats. J Appl Physiol. 2001;90(4):1219-26.

11. Wagner PD. The critical role of VEGF in skeletal muscle angiogenesis and blood flow. Biochem Soc Trans. 2011;39(6):1556-9.

12. Gustafsson T, Puntschart A, Kaijser L, et al. Exercise-induced expression of angiogenesis-related transcription and growth factors in human skeletal muscle. Am J Physiol. 1999;276(2 Pt 2):H679-85.

13. Tan KH, Cotterrell D, Sykes $\mathrm{K}$, et al. Exercise training for claudicants: changes in blood flow, cardiorespiratory status, metabolic functions, blood rheology and lipid profile. Eur J Vasc Endovasc Surg. 2000;20(1):72-8.

14. Yang HT, Ogilvie RW, Terjung RL. Training increases collateral-dependent muscle blood flow in aged rats. Am J Physiol. 1995;268(3 Pt 2):H1174-80.

15. Wahlberg E. Angiogenesis and arteriogenesis in limb ischemia. J Vasc Surg. 2003;38(1):198-203.

16. Leiner $\mathrm{T}, \mathrm{Nijenhuis} \mathrm{RJ,} \mathrm{Maki} \mathrm{JH,} \mathrm{et} \mathrm{al.} \mathrm{Use} \mathrm{of} \mathrm{a} \mathrm{three-station} \mathrm{phased} \mathrm{array}$ coil to improve peripheral contrast-enhanced magnetic resonance angiography. J Magn Reson Imaging. 2004;20(3):417-25.

17. Versluis B, Backes WH, van Eupen MG, et al. Magnetic resonance imaging in peripheral arterial disease: reproducibility of the assessment of morphological and functional vascular status. Invest Radiol. 2011;46(1):1124.

18. Oostendorp M, Post MJ,Backes WH. Vessel growth and function: depiction with contrast-enhanced MR imaging. Radiology. 2009;251(2):317-35.

19. Koelemay MJ, den Hartog D, Prins $M H$, et al. Diagnosis of arterial disease of the lower extremities with duplex ultrasonography. $\mathrm{Br} J$ Surg. 1996;83(3):404-9.

20. Strandness DE, Jr., Priest RE, Gibbons GE. Combined Clinical and Pathologic Study of Diabetic and Nondiabetic Peripheral Arterial Disease. Diabetes. 1964;13:366-72.

21. Chao CY, Cheing GL. Microvascular dysfunction in diabetic foot disease and ulceration. Diabetes Metab Res Rev. 2009;25(7):604-14. 
22. Duet M, Virally M, Bailliart O, et al. Whole-body (201)TI scintigraphy can detect exercise lower limb perfusion abnormalities in asymptomatic diabetic patients with normal Doppler pressure indices. Nucl Med Commun. 2001;22(9):949-54.

23. Gardner AW, Skinner JS, Cantwell BW, et al. Progressive vs single-stage treadmill tests for evaluation of claudication. Med Sci Sports Exerc. $1991 ; 23(4): 402-8$.

24. Mohajer K, Zhang H, Gurell D, et al. Superficial femoral artery occlusive disease severity correlates with MR cine phase-contrast flow measurements. J Magn Reson Imaging. 2006;23(3):355-60.

25. Versluis B, Dremmen MH, Nelemans PJ, et al. MRI of arterial flow reserve in patients with intermittent claudication: feasibility and initial experience. PLoS One. 2012;7(3):e31514.

26. de Vries M, Nijenhuis RJ, Hoogeveen RM, et al. Contrast-enhanced peripheral MR angiography using SENSE in multiple stations: feasibility study. J Magn Reson Imaging. 2005;21(1):37-45.

27. Prakash A, Garg R, Marcus EN, et al. Faster flow quantification using sensitivity encoding for velocity-encoded cine magnetic resonance imaging: in vitro and in vivo validation. J Magn Reson Imaging. 2006;24(3):676-82.

28. Hiatt WR, Regensteiner JG, Hargarten ME, et al. Benefit of exercise conditioning for patients with peripheral arterial disease. Circulation. 1990;81(2):602-9.

29. Kruidenier LM, Viechtbauer W, Nicolai SP, et al. Treatment for intermittent claudication and the effects on walking distance and quality of life. Vascular. 2012;20(1):20-35.

30. Watson L, Ellis B, Leng GC. Exercise for intermittent claudication. Cochrane Database Syst Rev. 2008(4):CD000990.

31. Wind J, Koelemay MJ. Exercise therapy and the additional effect of supervision on exercise therapy in patients with intermittent claudication. Systematic review of randomised controlled trials. Eur J Vasc Endovasc Surg. 2007;34(1):1-9.

32. Treat-Jacobson D, Henly SJ, Bronas UG, et al. The pain trajectory during treadmill testing in peripheral artery disease. Nurs Res. 2011;60(3 Suppl):S38-49.

33. Lawall H, Bramlage P, Amann B. Treatment of peripheral arterial disease using stem and progenitor cell therapy. J Vasc Surg. 2010. 


\section{CHAPTER 6}

34. Lawall H, Bramlage P, Amann B. Stem cell and progenitor cell therapy in peripheral artery disease. A critical appraisal. Thromb Haemost. 2010;103(4):696-709.

35. Lederman RJ, Mendelsohn FO, Anderson RD, et al. Therapeutic angiogenesis with recombinant fibroblast growth factor-2 for intermittent claudication (the TRAFFIC study): a randomised trial. Lancet. 2002;359(9323):2053-8.

36. Rajagopalan S, Mohler ER, 3rd, Lederman RJ, et al. Regional angiogenesis with vascular endothelial growth factor in peripheral arterial disease: a phase II randomized, double-blind, controlled study of adenoviral delivery of vascular endothelial growth factor 121 in patients with disabling intermittent claudication. Circulation. 2003;108(16):1933-8.

37. Wu WC, Mohler E, 3rd, Ratcliffe SJ, et al. Skeletal muscle microvascular flow in progressive peripheral artery disease: assessment with continuous arterial spin-labeling perfusion magnetic resonance imaging. J Am Coll Cardiol. 2009;53(25):2372-7.

38. Ledermann HP, Schulte AC, Heidecker HG, et al. Blood oxygenation leveldependent magnetic resonance imaging of the skeletal muscle in patients with peripheral arterial occlusive disease. Circulation. 2006;113(25):2929-35.

39. Thompson RB, Aviles RJ, Faranesh AZ, et al. Measurement of skeletal muscle perfusion during postischemic reactive hyperemia using contrastenhanced MRI with a step-input function. Magn Reson Med. 2005;54(2):289-98. 


\title{
CHAPTER 7
}

\section{MRI of arterial flow reserve in patients with}

\author{
intermittent claudication:
}

Feasibility and initial experience

B. Versluis, M.H.G. Dremmen, P.J. Nelemans, J.E. Wildberger, G.W.H. Schurink, T. Leiner, W.H. Backes

Published at PLos One, 2012:

Versluis B, Dremmen $\mathrm{MH}$, Nelemans PJ, et al. MRI of arterial flow reserve in patients with intermittent claudication: feasibility and initial experience. PLoS One. 2012;7(3):e31514 


\section{CHAPTER 7}

\section{Abstract}

\section{Objectives}

The aim of this work was to develop a MRI method to determine arterial flow reserve in patients with intermittent claudication and to investigate whether this method can discriminate between patients and healthy control subjects.

\section{Methods}

Ten consecutive patients with intermittent claudication and 10 healthy control subjects were included. All subjects underwent vector cardiography triggered quantitative 2D cine MR phase-contrast imaging to obtain flow waveforms of the popliteal artery at rest and during reactive hyperemia. Resting flow, maximum hyperemic flow and absolute flow reserve were determined and compared between the two groups by two independent MRI readers. Also, interreader reproducibility of flow measures was reported.

\section{Results}

Resting flow was lower in patients compared to controls $(4.9 \pm 1.6$ and $11.1 \pm 3.2 \mathrm{~mL} / \mathrm{s}$ in patients and controls, respectively $(p<0.01)$ ). Maximum hyperemic flow was $7.3 \pm 2.9$ and $16.4 \pm 3.2 \mathrm{~mL} / \mathrm{s}(\mathrm{p}<0.01)$ and the absolute flow reserve was $2.4 \pm 1.6$ and $5.3 \pm 1.3 \mathrm{~mL} / \mathrm{s}(\mathrm{p}<$ 0.01 ), respectively in patients and controls. The interreader coefficient of variation was below $10 \%$ for all measures in both patients and controls.

\section{Conclusions}

Quantitative 2D MR cine phase-contrast imaging is a promising method to determine flow reserve measures in patients with peripheral arterial disease and can be helpful to discriminate patients with intermittent claudication from healthy controls. 


\section{Introduction}

The hemodynamic significance of obstructive arterial lesions in peripheral arterial disease (PAD) can be assessed by blood flow velocity measurements (1-5). Nevertheless, in patients with intermittent claudication - stage II PAD according to the Fontaine classification - blood flow at rest might be near-normal depending on the severity of the obstructive lesion, or recover quickly to near-normal values after exercise due to collateral artery formation (6-13). These (near-)normal resting flow values explain the absence of clinical symptoms at rest in intermittent claudication. Symptoms in these patients, however, characteristically appear during physical exercise when the flow demands towards the lower extremity increase. Therefore the flow reserve, either defined as the absolute difference or relative value between maximum hyperemic flow and autoregulated resting flow, is expected to be compromised in patients with intermittent claudication (10, 14, 15). Arterial flow reserve correlates well with subjective severity of symptoms in intermittent claudication and is less hampered by day-to-day variations than resting flow (14). Also, flow reserve stays compromised for a prolonged period of time during the process of collateral formation (9). Moreover, it does not depend on the physical capabilities of the patient, such as with physical exercise tests and ankle-brachial index measurements. Flow reserve might therefore be a valuable additional objective measure of disease severity in PAD in those patients with normal resting flow values, in order to discriminate between hemodynamically significant and insignificant obstructive arterial lesions. Flow reserve could also be useful for therapy monitoring, since physical exercise tolerance is closely correlated with the flow reserve, in contrast to resting flow levels (7-9).

Flow reserve is usually measured with duplex ultrasonography. This method, however, is limited for the peripheral arteries by a relatively large interobserver variability during reactive hyperemic conditions, which are needed to determine the flow reserve $(16,17)$. Other techniques to measure blood flow are Laser Doppler flowmetry, con- 


\section{CHAPTER 7}

trast-enhanced ultrasound (CEUS) and intra-arterial catheter based flow measurements $(1,9,10,14,18)$. Unfortunately, these methods either lack the ability to directly measure flow in large, non-superficial, conduit arteries, are prone to poor interobserver variability, or are not suitable for repeated measurements, as in the context of therapy monitoring $(9,14,16)$. A promising alternative method to measure flow in the peripheral arteries, is MRI-based quantitative cine phase-contrast imaging (PCI) $(2-4,19,20)$. Cine $\mathrm{PCl}$ can easily be combined with standard contrast-enhanced MR angiograpy (CE-MRA) of the peripheral vasculature and therefore has the major advantage to provide highly accurate morphologic information $(21,22)$ together with relevant functional information in a single examination.

The purpose of the current study was (i) to develop a MRI method for the determination of vascular flow reserve in the popliteal artery (PA), using quantitative velocity encoded 2D MR cine $\mathrm{PCl}$ flow waveform measurements at rest and during reactive hyperemia, (ii) to evaluate the potential value of this method to discriminate patients with intermittent claudication from healthy control subjects, and (iii) to determine the reproducibility of measuring resting flow and flow reserve with cine $\mathrm{PCl}$.

\section{Material and methods}

\section{Subjects and Ethics statement}

Ten consecutive non-diabetic patients (mean age \pm SD: $66.5 \pm 9.9$ years; 8 males and 2 females) with clinical symptoms of PAD (Fontaine stage II; intermittent claudication with no signs of critical ischemia (23)) with suspected arterial lesions of the superficial femoral artery (SFA) by duplex ultrasonography (peak systolic velocity (PSV) ratio $>2.0$ (24)) were included, as well as 10 healthy control subjects with no known PAD symptoms (age $24.1 \pm 2.2$ years; 3 males and 7 females). Exclusion criteria were hemodynamic instability, contraindications for MRI (i.e. claustrophobia, known gadolinium based contrast agent allergy, or low estimated glomular filtration rate $\left.\left(<30 \mathrm{~mL} / \mathrm{kg} / 1.73 \mathrm{~m}^{2}\right)\right)$. The institu- 
tional medical ethics committee (METC azM/UM) approved the study and all subjects gave written informed consent before inclusion.

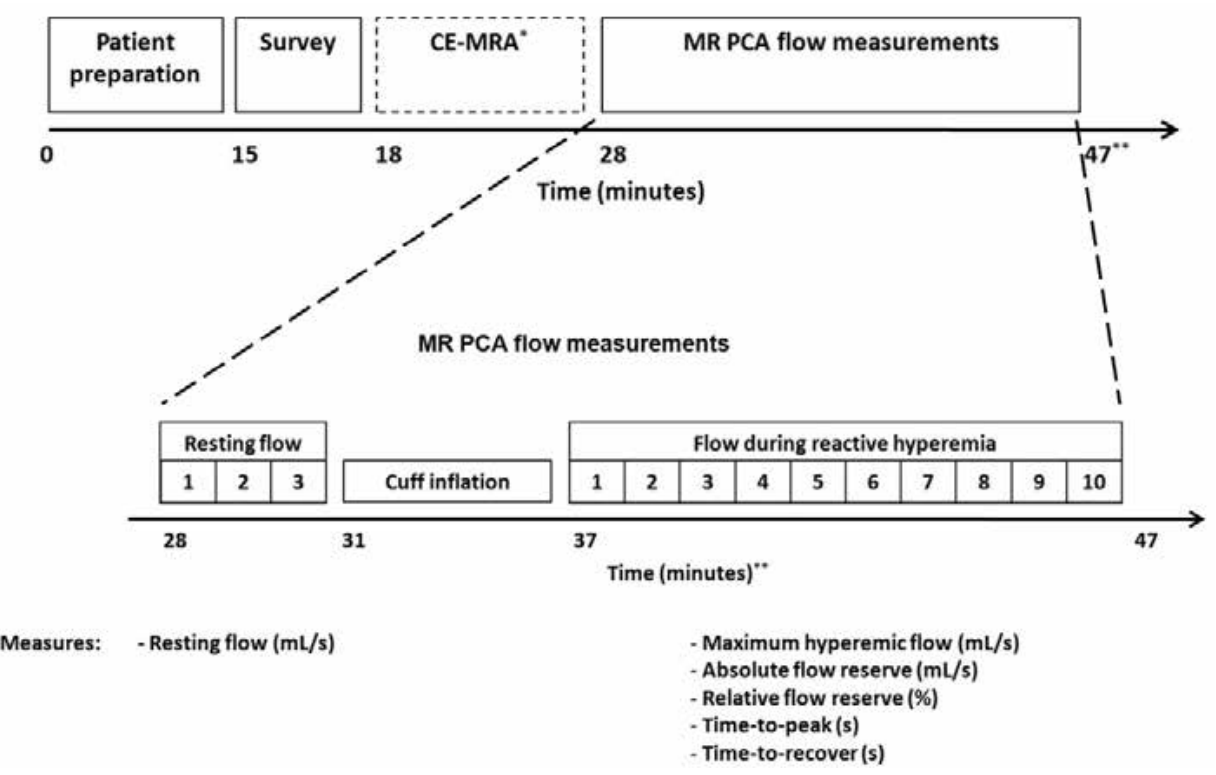

Figure 1. Overview of the imaging protocol. Three flow measurements were acquired at rest, before provoking reactive hyperemia to determine the average resting flow and reproducibility of resting flow. Reactive hyperemia was provoked by a cuff paradigm. After cuff deflation, 10 flow measurements were acquired during reactive hyperemia to determine the listed flow reserve measures.

" CE-MRA was performed in patients only. In healthy controls flow measurements started 10 minutes after the survey was completed.

${ }^{* *}$ Nominal scan duration at a regular heart rate of 60 beats per minute.

\section{MRI protocol}

All subjects underwent cine $\mathrm{PCl}$ measurements in the PA to obtain flow waveforms. Cine PCI was combined with CE-MRA of the peripheral arteries as part of the clinical routine examination. A schematic overview of the scan protocol is given in figure 1. All examinations were performed on a 1.5-T MRI system (Intera, Philips Medical Systems, Best, The Netherlands). For signal reception we used a dedicated 12-element phased-array peripheral vascular coil with a craniocaudal coverage of 


\section{CHAPTER 7}

$128 \mathrm{~cm}$ (Philips Medical Systems, Best, The Netherlands). Subjects were imaged in the supine position. All subjects were in this position for approximately 30 minutes before the first flow measurement was started. During this time a three-station CE-MRA was acquired in patients, using a fixed dose of $10 \mathrm{~mL}$ gadofosveset trisodium (Ablavar ${ }^{\circ}$, Lantheus Medical Imaging, Billerica, MA) as contrast agent.

In patients, flow was measured in the PA of the most symptomatic lower extremity. In all healthy controls flow was measured in the right PA. Postischemic reactive hyperemia in the lower leg was provoked using an inflatable cuff, ensuring total arterial occlusion (Medrad, indianola, PA). The cuff was placed at mid-thigh level and manually inflated to suprasystolic values $(>50 \mathrm{mmHg}$ above brachial systolic blood pressure) during 6 minutes. During cuff inflation, flow in the PA was measured with cine $\mathrm{PCl}$ to check that total arterial occlusion of the upper leg was achieved. PA was chosen as the vessel of interest as the $P A$ is the most distal artery with sufficiently large caliber to measure flow by $\mathrm{PCl}$ with a spatial resolution that enables fast cine $\mathrm{PCl}$ measurements $(19,20)$.

Survey. A non-enhanced time-of-flight (TOF) scan of the pelvic, upper and lower leg station was acquired to prescribe the imaging volumes of interest for morphological and functional imaging. A turbo field echo (TFE) pulse sequence was used with a $180^{\circ}$ inversion prepulse to suppress stationary tissue. Thirty-one axial slices per station were acquired with 3.3-mm slice thickness and 11-mm interslice gap, and an inferiorly concatenated saturation band. The standard quadrature body coil was used for signal transmission and reception. For the positioning of the 3D CE-MRA volumes maximum intensity projections (MIPs) were generated in 3 orthogonal directions.

CE-MRA. A three-station 3D FFE CE-MRA sequence was performed as previously described $(21,25)$. Acquisition parameters were as follows: TR $4.8 \mathrm{~ms}$, TE $1.45 \mathrm{~ms}$, flip angle $40^{\circ}$, FOV $470 \mathrm{~mm}$, matrix 


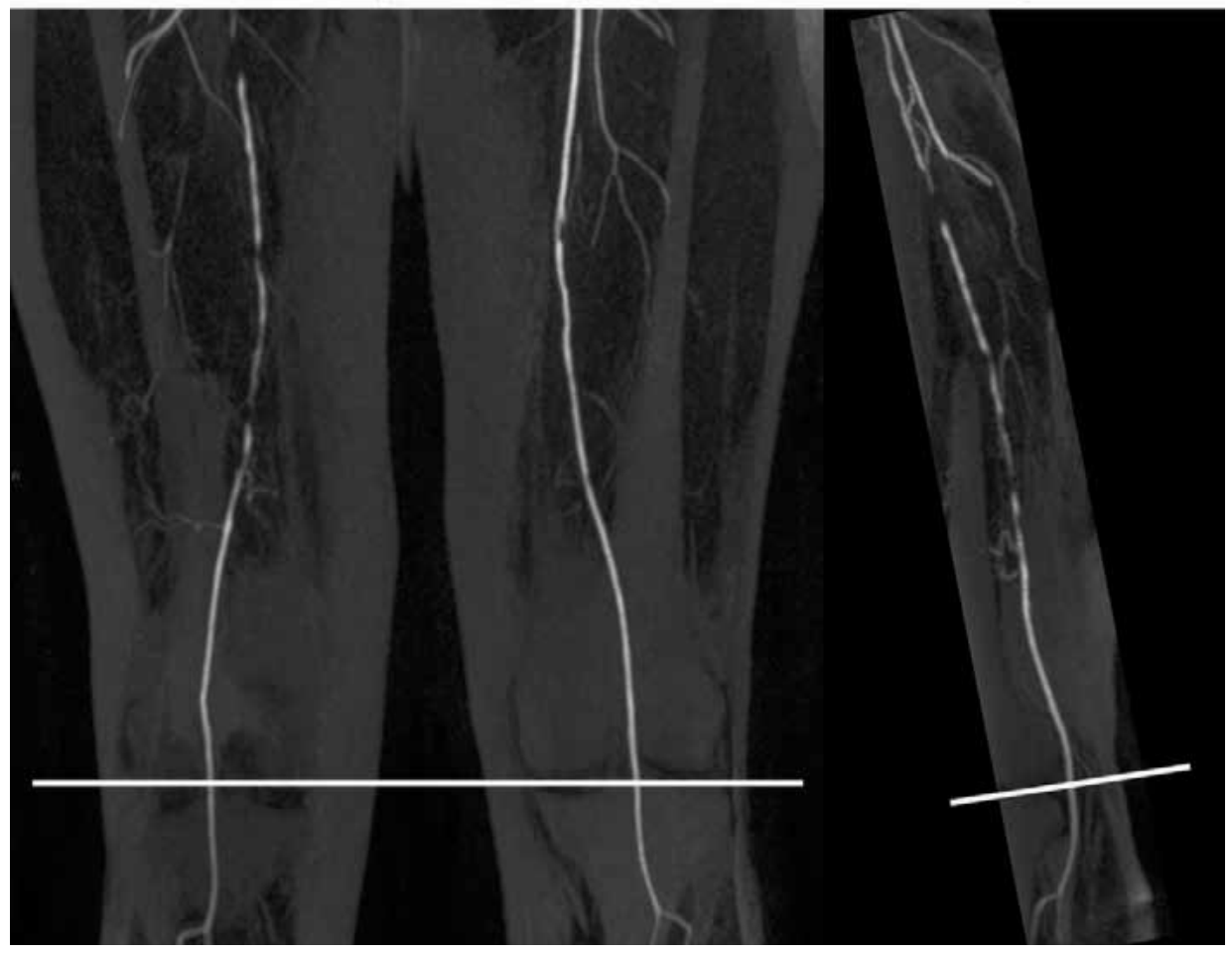

Figure 2. Coronal (A) and sagittal (B) reconstructions of CE-MRA of the upper leg of a PAD patient, showing the superficial femoral and popliteal artery. The cine $\mathrm{PCl}$ plane (line segments) was angulated perpendicular to the popliteal artery.

480 , and voxel dimensions (reconstructed) $0.92 \times 0.92 \times 1.20 \mathrm{~mm}$. Prior to contrast medium administration, a non-enhanced 'mask' image data set was acquired with exactly the same acquisition parameters as the CE-MRA, enabling background tissue suppression by image subtraction. For cine PCl we used a 2D FFE scan technique with the following acquisition parameters: TR $9.7 \mathrm{~ms}$, TE $5.8 \mathrm{~ms}$, flip angle $30^{\circ}$, FOV $380 \mathrm{~mm}$, matrix 384, and voxel dimensions (reconstructed) $0.99 \times 0.99 \times 6.00$ $\mathrm{mm}$ (26-28). Fifteen dynamic phases were acquired during the cardiac cycle. To focus on the peak velocity and flow, the phase encoding velocity (VENC) was set to $100 \mathrm{~cm} / \mathrm{s}$ in the craniocaudal direction, as published before $(2,29)$. Vector cardiography (VCG) triggering was used for retrospective cardiac synchronization. Parallel imaging (sensitivity 


\section{CHAPTER 7}

encoding, SENSE) was applied to reduce scan time (SENSE factor 2 in the anterior-posterior direction). At a mean heart rate of 60 beats per minute, the nominal acquisition time was 1 minute per dynamic acquisition. Coronal and sagittal MIP reconstructions of CE-MRA images were used for accurate positioning and angulation of the slice, perpendicular to the direction of the PA (see figure 2), for cine PCI measurement.

\section{Flow analysis}

Two independent MRI readers, blinded for each other's results, analyzed all datasets. Modulus and phase images were reconstructed from the cine $\mathrm{PCl}$ data. MRIcro (MRIcro, http://www.mricro.com/) was used as the image software application to manually draw a region of interest (ROI) covering the entire visible cross-section of the PA on each of the 15 reconstructed modulus images spanning the cardiac cycle. These regions of interest (ROIs) were analyzed, using self-written software code (Matlab, The Mathworks inc., Natick, MA) to obtain flow waveforms from the phase images (27). Phase images and flow waveforms were visually analyzed to detect possible aliasing due to a low phase encoding velocity. Flow waveforms were used to determine flow in $\mathrm{mL} / \mathrm{s}$ by integrating the $2 \mathrm{D}$ velocity profile over the cross-section of the artery. For this study, we primarily focused on the vascular stress condition, and therefore the arterial peak flow (related to peak velocity) was the primary functional quantity measured, rather than mean flow or velocity, cross-sectional luminal area or other velocity measures, as peak flow is physiologically the most relevant quantity and known to be most reproducible (2). Mean flow, peak velocity and peak area were nevertheless determined and can be found in appendix 1 .

Resting flow was defined as the average flow of three consecutive acquisitions at rest prior to cuff inflation. Absolute flow reserve was defined as the absolute difference between maximum hyperemic flow after cuff release and resting flow, whereas the relative flow reserve was defined as the ratio between these two measures. Also the time-to-peak (TTP), defined as the time needed to reach maximum hyperemic flow after cuff release, and time-to-recovery (TTR), defined as the time 
needed for flow to recover towards resting values after cuff release, were determined. An overview of the different flow parameters is illustrated in figure 3. Flow values were recorded as the average flow over a single dynamic measurement (approximately one minute at a heart rate of 60 beats per minute). For example, if the maximum hyperemic flow was observed during the first measurement after cuff release, a TTP of 30 seconds was recorded. If the maximum hyperemic flow was observed during the second measurement, a TTP of $60+30=90$ seconds was recorded. TTR was determined similarly.

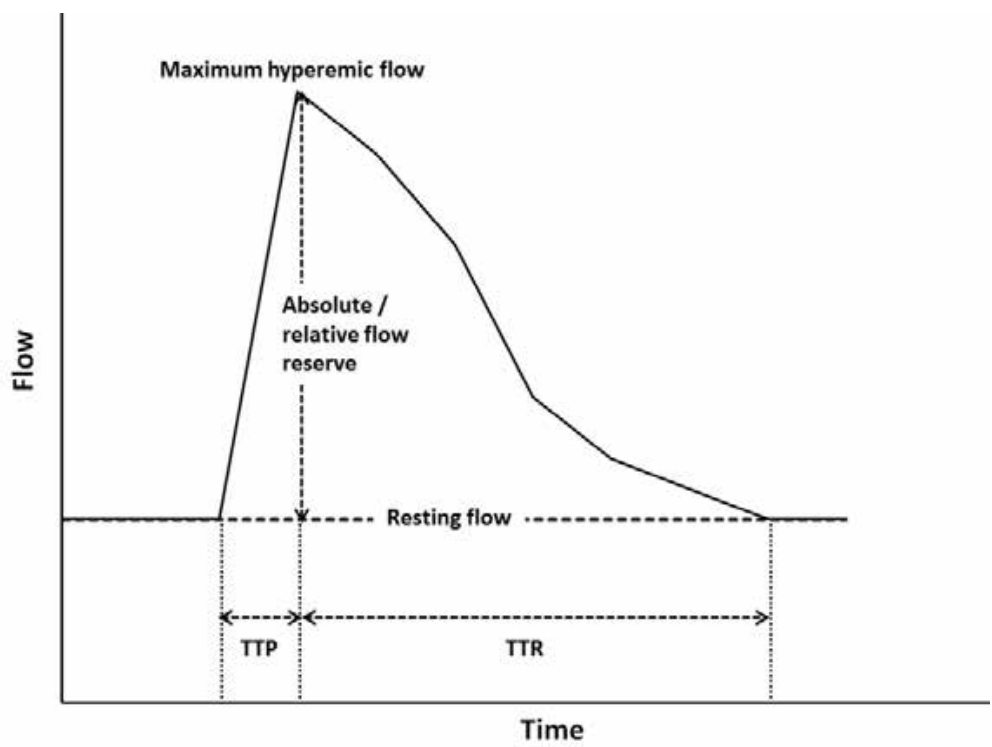

Figure 3. Overview of the different flow (reserve) measures. Absolute and relative flow reserve are defined as the absolute difference and the ratio between maximum hyperemic and resting flow, respectively. TTP, time-to-peak; TTR, time-to-recover.

\section{Statistical analysis}

Statistical analysis was performed with commercially available statistical software (SPSS 16.0, SPSS Inc., Chicago, IL). For the small groups a non-parametric 2-samples test (Mann-Whitney test) was applied to compare measures between patients and healthy controls. 


\section{ChAPTER 7}

$\mathrm{P}<0.05$ was considered statistically significant. Data of both MRI readers were averaged for the comparison between patients and healthy controls.

The variation of consecutive flow measurements at rest and the interreader reproducibility of resting flow and flow reserve measures were calculated. To determine interreader reproducibility, two measures of agreement were determined, being the coefficient of variation (CV in $\%$ ) and the repeatability coefficient (RC). In addition, the intraclass correlation coefficient (ICC) was determined as a parameter of reliability. The CV represents the relative variation within subjects and was derived by dividing the overall within-subject standard deviation $\left(\mathrm{SD}_{\mathrm{ws}}\right)$ by the mean measurement value over all subjects. The RC gives the smallest noticeable difference that can be detected beyond measurement error and is defined as $1.96 \cdot \sqrt{ } 2 \cdot \mathrm{SD}_{\text {ws }}(30,31)$. In other words, the difference between 2 measurements in the same subject is expected to be less than the RC in $95 \%$ of the observations in cases where the measured quantity remains unchanged over time. A value of RC lower than the absolute difference between mean values in patients and healthy controls indicates good agreement. The ICC, the fraction of total variance due to variation between subjects, rather than measurement error, was calculated using a 1-way random model, according to ICC $=\mathrm{SD}_{\mathrm{bs}^{2}}{ }^{2}$ / $\left(\mathrm{SD}_{\mathrm{bs}}{ }^{2}+\mathrm{SD}_{\mathrm{ws}}{ }^{2}\right)$, where $S \mathrm{D}_{\mathrm{bs}}$ represents the standard deviation between subjects. If the measurement error is small compared to the variation between subjects, the ICC approaches 1, i.e. reliability is very high. Interreader reproducibility was determined for the resting flow and both flow reserve measures.

\section{Results}

\section{Subjects}

All subjects included underwent cine PCl to obtain flow waveforms as planned and without experiencing side effects or adverse events. Total arterial occlusion during cuff compression could be confirmed by 
cine $\mathrm{PCl}$ measurements in all subjects. All cine $\mathrm{PCl}$ images were of sufficient quality for quantitative analysis and no aliasing was detected. The average heart rate (mean \pm SD) in patients was $67 \pm 9$ beats per minute (bpm), versus $62 \pm 11 \mathrm{bpm}$ in healthy controls (no significant difference, $p=0.23$ ).

\section{CE-MRA}

CE-MRA revealed significant stenosis ( $>50 \%$ ) of the SFA in 6 out of 10 patients, whereas long occlusions of the SFA were found in the remaining patients. Large collateral arteries were found in those patients with SFA occlusions, whereas in patients with significant stenosis of the SFA there were no prominent collateral arteries. Iliac artery vessel wall irregularities were found in 3 out of 10 patients. The remaining patients had no signs of obstructive lesions of the iliac arteries. At least 2 out of 3 main conduit arteries of the lower leg were free from obstructive lesions in all patients. CE-MRA revealed no signs of PAD in any of the control subjects.

\section{Patients versus healthy controls}

Representative phase-contrast images (modulus and phase), cross sectional velocity 2D velocity profiles and flow waveforms at rest and during reactive hyperemia in a patient and healthy control subject are shown in figure 4 and 5 respectively. Flow increased during reactive hyperemia in both patients and healthy controls. Flow waveforms under resting conditions were either tri- $(n=2), b i-(n=2)$ or monophasic $(n=6)$ in patients and triphasic in all healthy controls. During reactive hyperemia flow waveforms became monophasic for all patients, as seen in figure 5 , while flow waveforms remained triphasic for all healthy controls. Figure 6 shows temporal variations in flow before and after provoking reactive hyperemia in a patient and a healthy control. 


\section{ChAPTER 7}

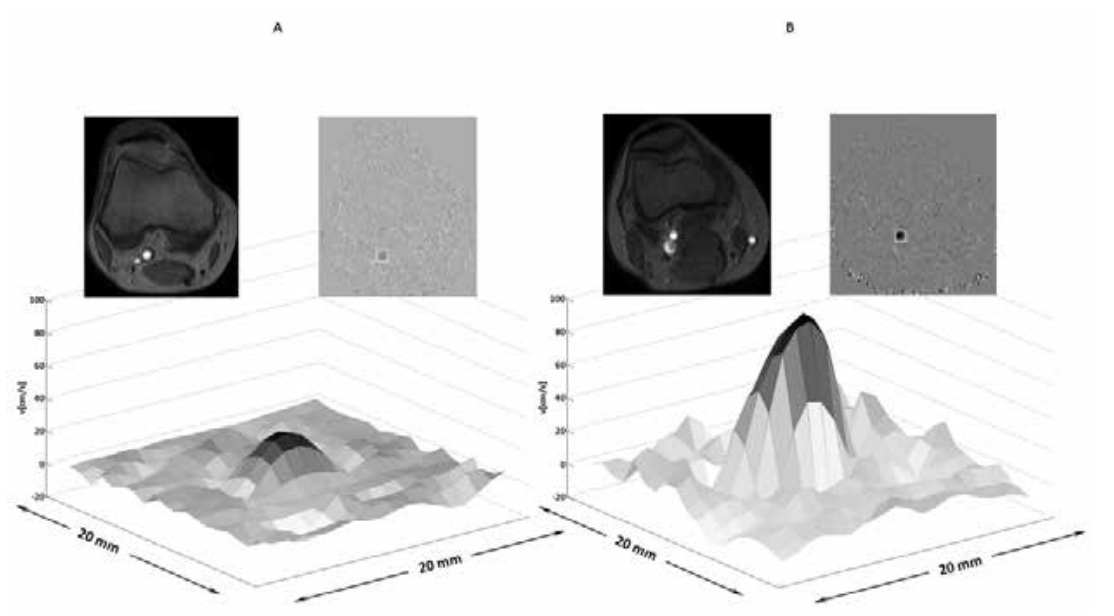

Figure 4. Example of $\mathrm{PCl}$ modulus images (left corner panel $\mathrm{A}$ and $\mathrm{B}$ ) and phase images (right corner panel $A$ and $B$ ) and the correspondingly measured 2D velocity profiles of a PAD patient (panel A) and healthy control (panel B). The brightest pixels of the modulus images represent the popliteal artery and is located within the white box on the phase images. 2D velocity profiles represent peak systolic velocity across the popliteal artery. Maximum peak velocity values were $24.3 \mathrm{~cm} / \mathrm{s}$ and $92.6 \mathrm{~cm} / \mathrm{s}$ respectively for the patient and healthy control, respectively.

A

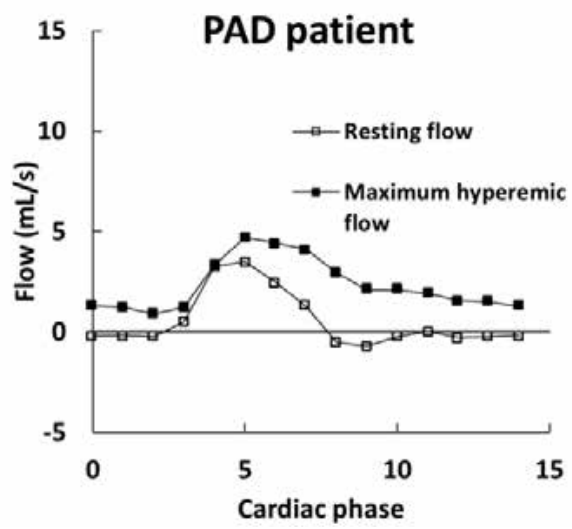

B

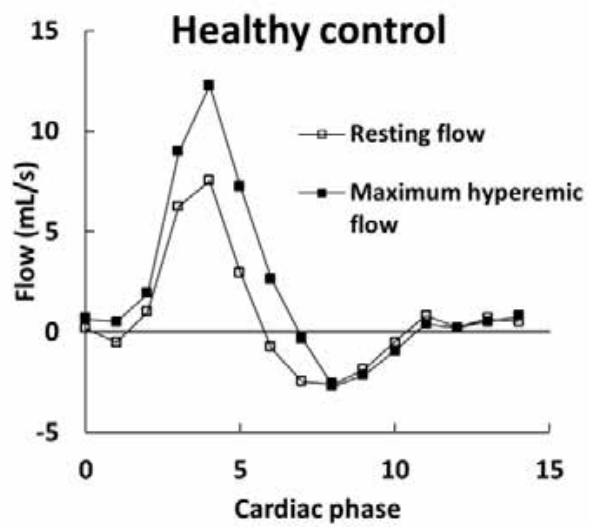

Figure 5. Flow waveforms in a PAD patient (panel A) and a healthy control (panel B) at rest and during maximum hyperemia. Note the mono-phasic flow waveform in the patient, both at rest and during reactive hyperemia. 


\begin{tabular}{cccc}
\hline & & \multicolumn{2}{c}{ Resting flow } \\
& & $\begin{array}{c}\text { Patients } \\
(\mathrm{n}=10)\end{array}$ & $\begin{array}{c}\text { Healthy controls } \\
(\mathrm{n}=10)\end{array}$ \\
\hline Value & $\mathrm{mL} / \mathrm{s}$ & $4.9 \pm 1.6$ & $11.1 \pm 3.2$ \\
Interreader reproducibility & & \\
$\mathrm{CV}$ & $\%$ & 4.0 & 4.3 \\
$\mathrm{RC}$ & $\mathrm{mL} / \mathrm{s}$ & 0.5 & 1.0 \\
$\mathrm{ICC}$ & $(95 \% \mathrm{Cl})$ & $0.99(0.96-0.99)$ & $0.99(0.96-0.99)$ \\
\hline
\end{tabular}

\begin{tabular}{|c|c|c|c|c|}
\hline & & & \multicolumn{2}{|c|}{ Maximum hyperemic flow } \\
\hline & & & Patients & Healthy controls \\
\hline Value & & $\mathrm{mL} / \mathrm{s}$ & $7.3 \pm 2.9$ & $16.4 \pm 3.2$ \\
\hline \multicolumn{5}{|c|}{ Interreader reproducibility } \\
\hline & CV & $\%$ & 4.6 & 3.5 \\
\hline & RC & $\mathrm{mL} / \mathrm{s}$ & 0.9 & 1.6 \\
\hline & ICC & $(95 \% \mathrm{Cl})$ & $0.99(0.95-0.99)$ & $0.97(0.89-0.99)$ \\
\hline
\end{tabular}

Absolute flow reserve

\begin{tabular}{lccc} 
& Patients & Healthy controls \\
\hline Value & $\mathrm{mL} / \mathrm{s}$ & $2.4 \pm 1.6$ & $5.3 \pm 1.3$
\end{tabular}

Interreader reproducibility

\begin{tabular}{cccc} 
CV & $\%$ & 9.8 & 8.7 \\
RC & $\mathrm{mL} / \mathrm{s}$ & 0.6 & 1.2 \\
$\mathrm{ICC}$ & $(95 \% \mathrm{Cl})$ & $0.98(0.93-0.99)$ & $0.89(0.63-0.97)$ \\
\hline
\end{tabular}

Relative flow reserve

\begin{tabular}{|c|c|c|c|c|}
\hline & & & Patients & Healthy controls \\
\hline Value & & $\%$ & $149 \pm 31$ & $151 \pm 19$ \\
\hline \multicolumn{5}{|c|}{ Interreader reproducibility } \\
\hline & $C V$ & $\%$ & 3.1 & 3.4 \\
\hline & RC & $\%$ & 12.5 & 14.0 \\
\hline & ICC & $(95 \% \mathrm{Cl})$ & $0.98(0.93-0.99)$ & $0.93(0.76-0.98)$ \\
\hline
\end{tabular}

Table 1. Flow measures and reproducibility in patients with intermittent claudication and healthy controls.

Values are presented as mean \pm SD. CV, coefficient of variation; RC, repeatability coefficient; ICC, intra-class correlation coefficient. 


\section{CHAPTER 7}

Absolute flow values at rest and during reactive hyperemia are given in table 1 . On average, resting flow in patients was less than half of the values measured in healthy controls $(p<0.01)$. Maximum hyperemic flow and absolute flow reserve in patients were also less than half the values found in healthy controls $(p<0.01)$. For relative flow reserve, on the other hand, there were no significant differences between patients and healthy controls $(p=0.81)$.

A

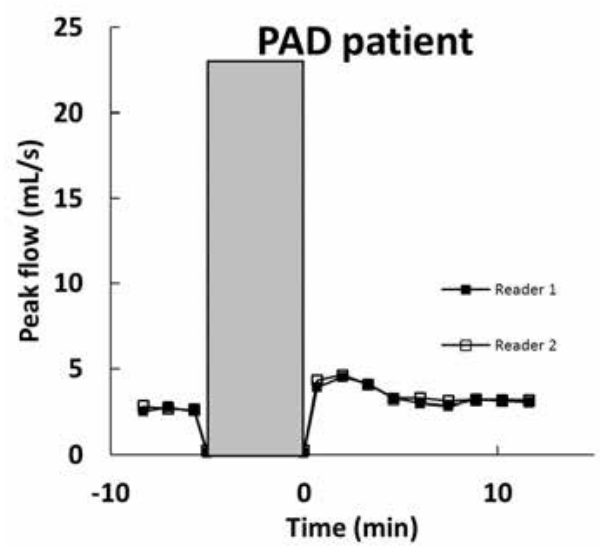

B

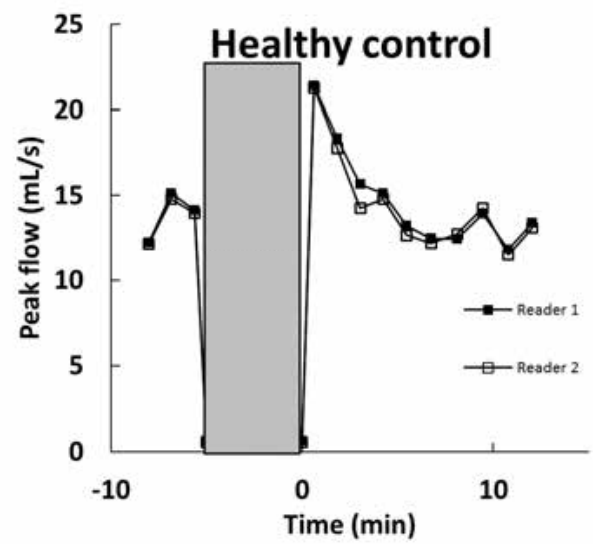

Figure 6. Peak flow in the popliteal artery before and after provoking reactive hyperemia by a cuff paradigm in a PAD patient (panel A) and a healthy control (panel B). The shaded bar represents the period of cuff compression to provoke reactive hyperemia. There is close agreement between the two MRI readers for both the patient and healthy control subject.

Figure 7 shows the distribution of resting flow and absolute flow reserve values in patients. The upper and lower limits represent mean values \pm 2 .SD in healthy controls. Resting flow was lower than the lower limit of healthy controls (i.e. mean - 2.SD) in 4 patients. Absolute flow reserve revealed lower values than the lower limit in 6 patients, of whom 2 patients had normal resting flow values (see cross-table of figure 7). In 4 patients both resting flow and flow reserve values were within the upper and lower limits as encountered in control subjects. 

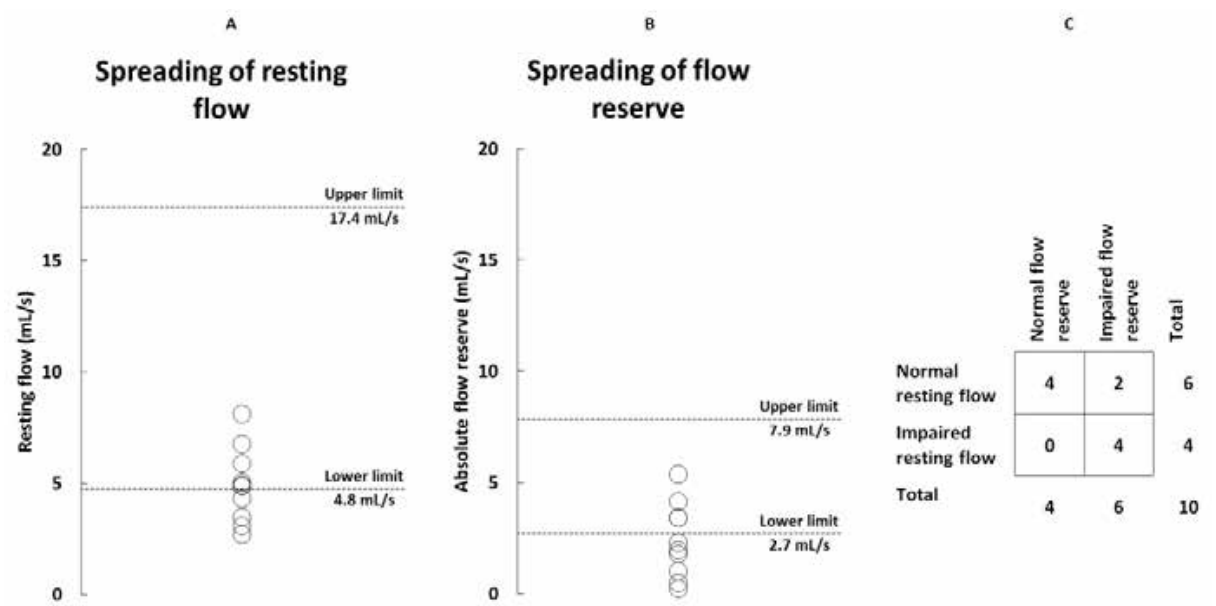

Figure 7. Resting flow (panel A) and absolute flow reserve (panel B) in PAD patients. The upper and lower limits in the graphics represent the mean value $\pm 2 \mathrm{SD}$ of resting peak flow (panel A) and absolute peak flow reserve (panel B) of healthy controls. The crosstable (right C) shows the number of patients with flow and flow reserve values within (normal values) or below (impaired values) the lower limits.

TTP was $65 \pm 52$ (mean \pm SD) seconds in patients and $35 \pm 29$ seconds in healthy controls. TTR was $360 \pm 212$ and $254 \pm 96$ seconds in patients and healthy controls, respectively. These results for TTP and TTR indicate a trend towards slower responses in patients $(p=0.15$ and $p=0.16$ for TTP and TTR, respectively).

\section{Vascular lesions and flow measures}

Resting flow in patients with an occlusion of the SFA was significantly lower compared to those with a significant stenosis $(p=0.02)$. Three out of 4 patients with an occlusion of the SFA had resting flow values lower than the lower limit of healthy controls.

Maximum hyperemic flow, absolute and relative flow reserve measures did not differ significantly between patients with occlusions and significant stenosis ( $p=0.15, p=0.72$ and $p=0.63$ respectively). Absolute flow reserve was lower than the lower limit of healthy controls in 3 out of 4 patients with a SFA occlusion and 3 out of 6 patients with a significant stenosis of the SFA. 


\section{ChAPTER 7}

\section{Reproducibility}

The variation between the three consecutive resting flow measurements was $4.2 \%$ in patients and $6.6 \%$ in healthy controls. Interreader reproducibility values of the flow measures are given in table S1. Interreader reproducibility for resting flow and flow reserve measures was high in both patients and healthy controls, with a CV below $10 \%$ for all flow measures in both groups (table 1). The RC values were smaller than the difference between PAD patients and healthy controls for the resting flow, maximum hyperemic flow, and the absolute flow reserve measures, but not for relative flow reserve.

\section{Discussion}

In this study we describe a simple MRI-based cine phase contrast imaging method that can be used to determine flow reserve measures in the popliteal artery in a vascular stress condition. Our initial results indicate that this a stable method that seems to be more sensitive in detecting peripheral arterial disease than resting flow measurements only.

Objective assessment of the functional consequences of stenoses and occlusions in the peripheral arterial tree remains an area of high interest for physicians dealing with PAD patients. Currently, the anklebrachial index (ABI) measurement is the most recognized and most widely applied functional measurement used for diagnosis and therapy monitoring $(5,32,33)$. ABI measurements, however, are hampered by poor reproducibility $(14,34)$. Besides, in patients with heavily calcified arteries in the lower leg, as frequently seen in patients with PAD, the ABI cannot be determined accurately or not be determined at all $(14,34$, 35).

The current study demonstrates that it is feasible to combine the ability of MRI to depict peripheral vascular anatomy with functional information (i.e. hemodynamic consequences) of popliteal artery flow reserve. Combining these two measurements in one examination is of 
high clinical interest, as it would potentially enable more objective assessment of PAD in patients suspected of having PAD, but also in the context of evaluating novel therapeutic strategies for PAD.

\section{Patients versus healthy controls}

Maximum hyperemic flow and absolute peak flow reserve were approximately $50 \%$ and significantly lower in patients compared to healthy controls. This shows that flow reserve measures can be used to determine the hemodynamic consequences of obstructive arterial lesions in the superficial femoral artery in PAD patients. These results are in line with previous studies and prove that the concept of blood flow reserve, originally introduced by Coffman and Gregg in 1960 for the coronary arteries (15), also seem to apply to peripheral arteries (9, $10,14,36,37)$. Lower flow reserve in the popliteal artery of PAD patients can be explained by the presence of obstructive arterial lesions, resulting in a decrease of the pressure gradient distal to the vascular lesion and thereby a decrease of the maximum hyperemic flow distal to these lesions (37).

An important observation is that, at group level, resting flow in this study was significantly reduced in patients with intermittent claudication, which concurs with previous studies $(2,29)$. This might suggest that resting flow alone already provides sufficient functional information on the hemodynamic significance of obstructive arterial lesions in intermittent claudication, without the need for flow reserve assessment. However, when looking at the resting flow values of individual patients (figure 7), it becomes clear that only four patients (40\%) had resting flow values below the mean flow $-2 \cdot S D$ in healthy controls. Three out of these four patients had a long occlusion of the SFA at CE-MRA. Regardless of the small number of patients, these initial results show that the type of vascular obstruction (i.e. stenosis or occlusion) affects resting flow. Although resting flow of these patients was lower than the lower limit in healthy controls, flow was still sufficient to sustain minimal perfusion of the lower leg as there were no clinical signs of ischemia at rest. Absolute flow reserve, on the other hand, was below the mean 


\section{ChAPTER 7}

value in healthy controls - 2 .SD in six (60\%) patients and was therefore able to identify two more patients with PAD compared to resting flow alone. Of these six patients, three suffered from an occlusion of the SFA and three had a significant stenosis of the SFA on CE-MRA. In the four remaining patients both resting flow and flow reserve appeared normal. This suggests that either no hemodynamically significant obstructive arterial lesions were present, or flow through the collateral arteries mitigated the effects of the stenoses.

Our initial results also indicate that for patients with (near-)normal resting flow values, assessment of the flow reserve might provide important additional functional information with respect to the hemodynamic significance of the arterial lesions, allowing to discriminate patients with intermittent claudication from healthy controls. Further studies with larger populations will be needed to determine the additional clinical value of flow reserve in the assessment of the hemodynamic significance of obstructive arterial lesions, both in patients with intermittent claudication and more severe stages of PAD.

Relative flow reserve, also known as the dimensionless flow reserve ratio in literature (37), was not significantly different between patients and healthy controls in this study, although we expected it to be impaired in patients. This discrepancy can be explained by the fact that resting and maximum hyperemic flow in patients as well as volunteers were reduced to approximately the same extent, resulting in a ratio for patients comparable to that in healthy controls. Based on our results there seems no clinical value of the relative flow reserve.

TTP and TTR did not significantly differ between patients and volunteers. However, when looking at the absolute values, it becomes clear that TTP and TTR are prolonged in patients, as we expected. However, the fact that we did not find significant differences in TTP and TTR between patients and healthy controls might be due to the relative small number of subjects. In addition, the low temporal resolution of approximately one minute per acquisition probably had a negative influence upon the discriminative ability for TTP and TTR as well. Previous studies on microvascular reactions during reactive hyperemia found prolonged 
peak and recovery times for microvascular flow between PAD patients and controls and the same phenomenon might be expected for macrovascular flow (38-40).

\section{Reproducibility}

Flow at rest was measured three times to determine an average resting flow. The variation between consecutive flow measurements at rest was very low in both patients (4.2\%) and healthy controls (6.6\%). This means that resting flow in a lying subject is relatively stable over time.

Resting flow and flow reserve measures (maximum hyperemic flow, absolute and relative flow reserve) all showed a high interreader reproducibility, with a interreader $\mathrm{CV}<5 \%$ for most measures (with exception of the absolute flow reserve in patients with a CV $<10 \%$ ). This indicates a low observer dependency of the measurements, which is desirable for clinical application in therapy monitoring and follow-up studies. The interreader CV is comparable to the variation of the (resting) flow measurements.

\section{Peak versus mean flow}

In this study peak flow values were preferred over mean flow values. Peak flow, the maximum flow during systole, is an attractive flow measure, as it correlates with the $A B I$ and is an important determinant of the systolic blood pressure (41). The decision to use peak flow was based on previous results, suggesting that peak values are more reproducible in cine $\mathrm{PCl}$ of the popliteal artery and show larger differences between PAD patients and healthy controls (2). Results on mean flow, peak velocity and peak area are presented in table 2 and discussed in appendix S1.

It would be interesting to see how well MRI derived flow reserve correlate and reproducibility measures compare with duplex ultrasonography derived flow measurements. Such a comparison, however, was beyond the scope of the current feasibility study and requires a larger 


\section{ChAPTER 7}

sample size and patient population. Besides, at present flow reserve is not yet routinely used in clinical practice for establishing the diagnosis and severity of PAD, neither using duplex ultrasonography nor MRI.

\section{Spatiotemporal resolution}

Previously published cine $\mathrm{PCl}$ protocols that measure flow reported a temporal resolution of 2 minutes or more $(2,42-44)$. We believe that this temporal resolution would have been insufficient to accurately measure the maximum flow during reactive hyperemia, as it is known that the maximum flow after cuff release is reached within one minute (45). Therefore, the temporal resolution of cine $\mathrm{PCl}$ measurements in the current study was improved by using parallel imaging (sensitivity encoding, SENSE $(46,47)$ ). Prakash et al (47) found good agreement between conventional cine $\mathrm{PCl}$ measurements and flow measurements with a parallel imaging acceleration factor of 2 , resulting in approximately $50 \%$ reduction in scan time. The results of our study show that in healthy controls the maximum hyperemic flow indeed was reached within one minute for every subject. To accurately measure the time-to-peak value in healthy subjects, and probably patients with less severe forms of PAD, further improvement of the temporal resolution is required. In PAD patients however, the time-to-peak was markedly longer, and in most patients the maximum hyperemic flow was not reached during the first measurement after cuff deflation. The currently used temporal resolution therefore seems to be sufficient for the application of this method in patients. Nevertheless, further improvement of the temporal resolution might result in more accurate (and possibly higher, as the measured value is an average flow measure over the entire duration of the measurement) flow reserve values in healthy persons, which will increase the ability to discriminate more patients from healthy controls. With our current equipment, however, parallel imaging acceleration factors above 2 results in a strong decrease in image quality.

Along the same vein, the temporal resolution of the waveform acquisition and the accuracy of the flow values can be improved, particu- 
larly in healthy controls, by applying faster image sampling. However, a present drawback is that spatial resolution will consequently decrease.

\section{Conclusion}

This exploratory study demonstrates that quantitative 2D MR cine $\mathrm{PCl}$ flow waveform measurement in the popliteal artery is a simple and stable method to assess the functional severity of arterial stenoses in patients with intermittent claudication. A strong reduction in maximum hyperemic flow and absolute flow reserve was found in patients with intermittent claudication compared to healthy controls. Therefore, assessment of flow reserve might be a valuable addition to MR angiography to objectively determine the hemodynamic consequences and disease severity in PAD patients. 


\section{Appendix}

In addition to arterial peak flow also mean flow, peak velocity and area were determined and are listed in table 2 .

Mean flow. No significant differences for mean flow were found between patients and healthy controls (table 2). The results in table 2 also confirm that interreader reproducibility is markedly lower for mean flow as compared to peak flow. Previous studies already revealed better discriminative capabilities and reproducibility for arterial peak flow over mean flow, which is confirmed in the current study $(2,29)$. Poor interreader reproducibility for mean flow is probably induced by low contrast between the lumen of the popliteal artery during diastole and static tissue, which makes it difficult to correctly identify vessel contours. Also, the applied acquisition settings, especially the relatively high phase encoding velocity (VENC) of $100 \mathrm{~cm} / \mathrm{s}$ is less suitable to accurately measure low flow velocities during diastole in the popliteal artery. Nevertheless, we choose this VENC value as we were primarily focused on peak flow. A venc value of $100 \mathrm{~cm} / \mathrm{s}$ was approx. 30-40\% above the highest peak velocity values we found at rest in healthy controls in a previous study (29), and we expected this value to be high enough to measure the maximum hyperemic peak velocity. We decided to use a fixed (i.e. predefined and constant) phase encoding velocity for both patients and controls as we (i) we did not know the optimal phase encoding velocity for each patient (especially during hyperemia) prior to the study, and (ii) in clinical practice, a fixed phase encoding velocity would be highly desirable for reasons of time efficiency, and also (iii) by using a constant phase encoding velocity the methodology was identical for each subject.

Peak velocity. Peak velocity results are concordant to peak flow results, with resting peak velocity, maximum hyperemic peak velocity and absolute peak velocity reserve being significantly lower in patients $(p<$ 0.01 ), whereas no significant difference was found for relative peak velocity reserve. Interreader reproducibility is excellent, which is hardly surprising, as during data analysis it is easy to identify the intraluminal 
voxel with the highest velocity (i.e at the vessel's center). Nevertheless, in clinical practice the use of peak velocity might be problematic. Arterial peak velocity is known to increase over hemodynamically significant stenotic vessel segments (an increase that is measured clinically by determining the peak systolic velocity (PSV)-ratio by ultrasound (48)). As far as peak flow is concerned, the increase in arterial peak flow is largely neutralized by the decrease in area at the stenosis. Moreover, during hyperemia, the arterial peak velocity only measures the increase in velocity, but not the influence of vasodilation. By measuring arterial peak flow, both the increase in velocity and vasodilatation are considered, which makes peak flow a more robust and physiologically more relevant measure. In addition peak velocity is much more sensitive to errors in angulation between imaging plane and vessel axis. Small errors in non-perpendicular orientation between vessel and imaging plane will have no impact upon flow as cross-sectional area increases compensate the decreases in the axial velocity component.

Cross-sectional area. Vessel cross-sectional area showed no significant differences between patients and healthy controls and is therefore not useful to discriminate patients with PAD from healthy controls 


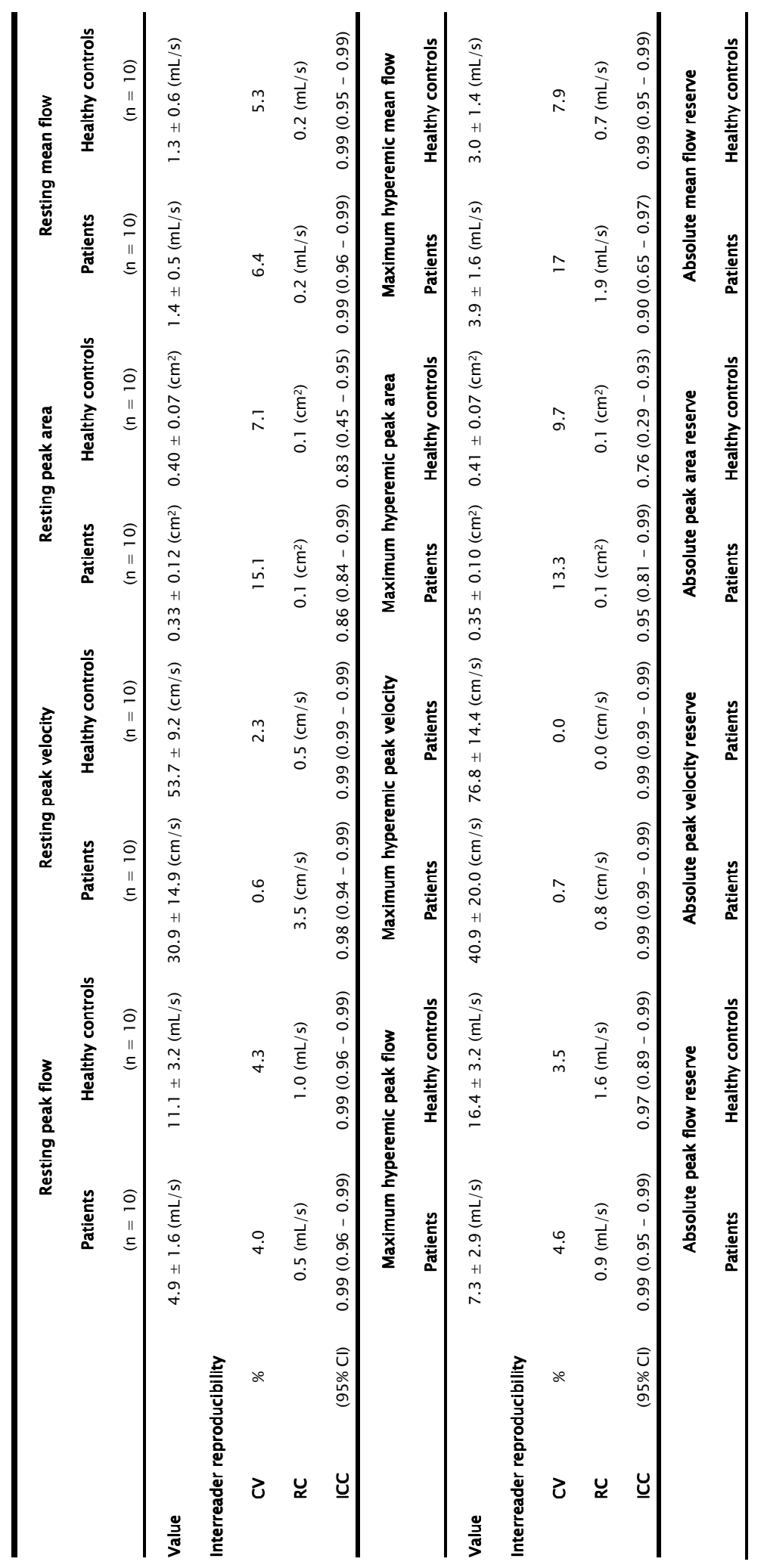




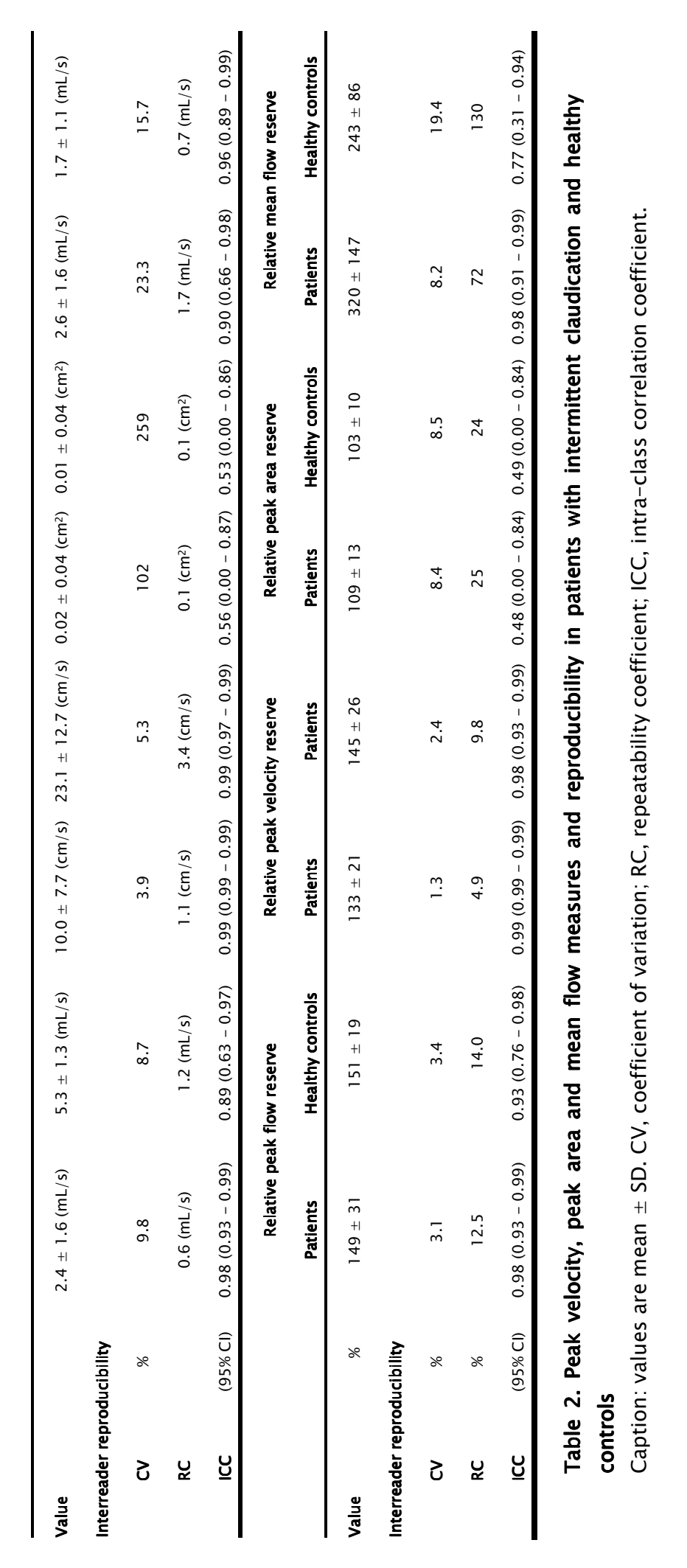




\section{References}

1. Begelman SM, Jaff MR. Noninvasive diagnostic strategies for peripheral arterial disease. Cleve Clin J Med. 2006;73 Suppl 4:S22-9.

2. Mohajer K, Zhang $\mathrm{H}$, Gurell $\mathrm{D}$, et al. Superficial femoral artery occlusive disease severity correlates with MR cine phase-contrast flow measurements. J Magn Reson Imaging. 2006;23(3):355-60.

3. Klein WM, Bartels LW, Bax L, et al. Magnetic resonance imaging measurement of blood volume flow in peripheral arteries in healthy subjects. J Vasc Surg. 2003;38(5):1060-6.

4. Meyer RA, Foley JM, Harkema SJ, et al. Magnetic resonance measurement of blood flow in peripheral vessels after acute exercise. Magn Reson Imaging. 1993;11(8):1085-92.

5. Norgren L, Hiatt WR, Dormandy JA, et al. Inter-Society Consensus for the Management of Peripheral Arterial Disease (TASC II). J Vasc Surg. 2007;45 Suppl S:S5-67.

6. Kinney TB, Rose SC. Intraarterial pressure measurements during angiographic evaluation of peripheral vascular disease: techniques, interpretation, applications, and limitations. AJR Am J Roentgenol. 1996;166(2):277-84.

7. Mathien GM, Terjung RL. Muscle blood flow in trained rats with peripheral arterial insufficiency. Am J Physiol. 1990;258(3 Pt 2):H759-65.

8. Brevetti LS, Paek R, Brady SE, et al. Exercise-induced hyperemia unmasks regional blood flow deficit in experimental hindlimb ischemia. J Surg Res. 2001;98(1):21-6.

9. Corcoran HA, Smith BE, Mathers P, et al. Laser Doppler imaging of reactive hyperemia exposes blood flow deficits in a rat model of experimental limb ischemia. J Cardiovasc Pharmacol. 2009;53(6):446-51.

10. Bragadeesh T, Sari I, Pascotto M, et al. Detection of peripheral vascular stenosis by assessing skeletal muscle flow reserve. J Am Coll Cardiol. 2005;45(5):780-5.

11. May AG, Van De Berg L, Deweese JA, et al. Critical arterial stenosis. Surgery. 1963;54:250-9.

12. Pena CS, McCauley TR, Price TB, et al. Quantitative blood flow measurements with cine phase-contrast MR imaging of subjects at rest and after exercise to assess peripheral vascular disease. AJR Am J Roentgenol. 1996;167(1):153-7. 
13. Hudlicka O, Brown MD. Adaptation of skeletal muscle microvasculature to increased or decreased blood flow: role of shear stress, nitric oxide and vascular endothelial growth factor. J Vasc Res. 2009;46(5):504-12.

14. Lindner JR, Womack L, Barrett EJ, et al. Limb stress-rest perfusion imaging with contrast ultrasound for the assessment of peripheral arterial disease severity. JACC Cardiovasc Imaging. 2008; 1(3):343-50.

15. Coffman JD, Gregg DE. Reactive hyperemia characteristics of the myocardium. Am J Physiol. 1960;199:1143-9.

16. van Langen $\mathrm{H}$, van Gurp J, Rubbens L. Interobserver variability of anklebrachial index measurements at rest and post exercise in patients with intermittent claudication. Vasc Med. 2009;14(3):221-6.

17. Hadlock J, Beach KW. Velocity variability in ultrasonic Doppler examinations. Ultrasound Med Biol. 2009;35(6):949-54.

18. Kido M, Takeuchi S, Hayashida S, et al. Assessment of abnormal blood flow and efficacy of treatment in patients with systemic sclerosis using a newly developed microwireless laser Doppler flowmeter and arm-raising test. Br J Dermatol. 2007;1 57(4):690-7.

19. Dumoulin CL. Phase contrast MR angiography techniques. Magn Reson Imaging Clin N Am. 1995;3(3):399-411.

20. Walker MF, Souza SP, Dumoulin CL. Quantitative flow measurement in phase contrast MR angiography. J Comput Assist Tomogr. 1988;12(2):304-13.

21. de Vries M, Nijenhuis RJ, Hoogeveen RM, et al. Contrast-enhanced peripheral MR angiography using SENSE in multiple stations: feasibility study. J Magn Reson Imaging. 2005;21(1):37-45.

22. Leiner $\mathrm{T}$, Kessels AG, Nelemans PJ, et al. Peripheral arterial disease: comparison of color duplex US and contrast-enhanced MR angiography for diagnosis. Radiology. 2005;235(2):699-708.

23. Aslam F, Haque A, Foody J, et al. Peripheral arterial disease: current perspectives and new trends in management. South Med J. 2009;102(11):1141-9.

24. Koelemay MJ, den Hartog D, Prins $M H$, et al. Diagnosis of arterial disease of the lower extremities with duplex ultrasonography. $\mathrm{Br} J$ Surg. 1996;83(3):404-9.

25. Leiner $\mathrm{T}$, Nijenhuis RJ, Maki JH, et al. Use of a three-station phased array coil to improve peripheral contrast-enhanced magnetic resonance angiography. J Magn Reson Imaging. 2004;20(3):41 7-25. 


\section{ChAPTER 7}

26. Bakker CJ, Hoogeveen RM, Viergever MA. Construction of a protocol for measuring blood flow by two-dimensional phase-contrast MRA. J Magn Reson Imaging. 1999;9(1):119-27.

27. Hoogeveen RM, Bakker CJ, Viergever MA. MR phase-contrast flow measurement with limited spatial resolution in small vessels: value of modelbased image analysis. Magn Reson Med. 1999;41(3):520-8.

28. Machida $\mathrm{H}$, Komori $\mathrm{Y}$, Ueno $\mathrm{E}$, et al. Accurate measurement of pulsatile flow velocity in a small tube phantom: comparison of phase-contrast cine magnetic resonance imaging and intraluminal Doppler guidewire. Jpn J Radiol. 2010;28(8):571-7.

29. Versluis B, Backes WH, van Eupen MG, et al. Magnetic resonance imaging in peripheral arterial disease: reproducibility of the assessment of morphological and functional vascular status. Invest Radiol. 2011;46(1):1124.

30. Bland JM, Altman DG. Measurement error. BMJ. 1996;312(7047):1654.

31. Jansen JF, Kooi ME, Kessels AG, et al. Reproducibility of quantitative cerebral T2 relaxometry, diffusion tensor imaging, and $1 \mathrm{H}$ magnetic resonance spectroscopy at 3.0 Tesla. Invest Radiol. 2007;42(6):327-37.

32. Hirsch AT, Haskal ZJ, Hertzer NR, et al. ACC/AHA 2005 Practice Guidelines for the management of patients with peripheral arterial disease (lower extremity, renal, mesenteric, and abdominal aortic): a collaborative report from the American Association for Vascular Surgery/Society for Vascular Surgery, Society for Cardiovascular Angiography and Interventions, Society for Vascular Medicine and Biology, Society of Interventional Radiology, and the ACC/AHA Task Force on Practice Guidelines (Writing Committee to Develop Guidelines for the Management of Patients With Peripheral Arterial Disease): endorsed by the American Association of Cardiovascular and Pulmonary Rehabilitation; National Heart, Lung, and Blood Institute; Society for Vascular Nursing; TransAtlantic Inter-Society Consensus; and Vascular Disease Foundation. Circulation. 2006;113(11):e463-654.

33. Creager MA. Clinical assessment of the patient with claudication: the role of the vascular laboratory. Vasc Med. 1997;2(3):231-7.

34. Stein R, Hriljac I, Halperin JL, et al. Limitation of the resting ankle-brachial index in symptomatic patients with peripheral arterial disease. Vasc Med. 2006;11(1):29-33.

35. Al-Qaisi M, Nott DM, King DH, et al. Ankle brachial pressure index (ABPI): An update for practitioners. Vasc Health Risk Manag. 2009;5:833-41. 
36. Hoffman Jl. Maximal coronary flow and the concept of coronary vascular reserve. Circulation. 1984;70(2):153-9.

37. Galderisi M, D'Errico A. Beta-blockers and coronary flow reserve: the importance of a vasodilatory action. Drugs. 2008;68(5):579-90.

38. Thompson RB, Aviles RJ, Faranesh AZ, et al. Measurement of skeletal muscle perfusion during postischemic reactive hyperemia using contrastenhanced MRI with a step-input function. Magn Reson Med. 2005;54(2):289-98.

39. Ledermann HP, Schulte AC, Heidecker HG, et al. Blood oxygenation leveldependent magnetic resonance imaging of the skeletal muscle in patients with peripheral arterial occlusive disease. Circulation. 2006;113(25):2929-35.

40. Duteil S, Wary C, Raynaud JS, et al. Influence of vascular filling and perfusion on BOLD contrast during reactive hyperemia in human skeletal muscle. Magn Reson Med. 2006;55(2):450-4.

41. Bishara RA, Taha W, Alfarouk MO, et al. Duplex detected ankle peak systolic velocity: a new parameter for the assessment of degree of peripheralischemia. Int Angiol. 2004;23(4):368-72.

42. Bakker CJ, Kouwenhoven M, Hartkamp MJ, et al. Accuracy and precision of time-averaged flow as measured by nontriggered 2D phase-contrast MR angiography, a phantom evaluation. Magn Reson Imaging. 1995;13(7):959-65.

43. Box FM, Spilt A, Van Buchem MA, et al. Automatic model-based contour detection and blood flow quantification in small vessels with velocity encoded magnetic resonance imaging. Invest Radiol. 2003;38(9):567-77.

44. Henk CB, Grampp S, Backfrieder W, et al. Automated vessel edge detection in velocity-encoded cine-MR (VEC-MR) flow measurements: a retrospective evaluation in critically ill patients. Eur J Radiol. 2003;48(3):274-81.

45. Brener BJ, Raines JK, Darling RC, et al. Measurement of systolic femoral aterial pressure during reactive hyperemia. An estimate of aortoiliac disease. Circulation. 1974;50(2 Suppl):II259-67.

46. Pruessmann KP, Weiger $M$, Scheidegger MB, et al. SENSE: sensitivity encoding for fast MRI. Magn Reson Med. 1999;42(5):952-62.

47. Prakash A, Garg R, Marcus EN, et al. Faster flow quantification using sensitivity encoding for velocity-encoded cine magnetic resonance imaging: in vitro and in vivo validation. J Magn Reson Imaging. 2006;24(3):676-82. 


\section{Chapter 7}

48. de Smet AA, Ermers EJ, Kitslaar PJ. Duplex velocity characteristics of aortoiliac stenoses. J Vasc Surg. 1996;23(4):628-36. 


\title{
CHAPTER 8
}

\section{Dynamic contrast-enhanced MRI assessment of}

\author{
hyperemic fractional microvascular blood
}

plasma volume in peripheral arterial disease:

Initial findings

B. Versluis, M.H.G. Dremmen, P.J. Nelemans, J.E. Wildberger, G.W.H. Schurink, T. Leiner, W.H. Backes

Published at PLos One, 2012:

Versluis B, Dremmen $\mathrm{MH}$, Nelemans PJ, et al. Dynamic contrastenhanced MRI assessment of hyperemic fractional microvascular blood plasma volume in peripheral arterial disease: initial findings. PLoS One. 2012;7(5):e37756 


\section{Abstract}

\section{Objectives}

The aim of the current study was to describe a method that assesses the hyperemic microvascular blood plasma volume of the calf musculature. The reversibly albumin binding contrast agent gadofosveset was used in dynamic contrast-enhanced magnetic resonance imaging (DCE MRI) to assess the microvascular status in patients with peripheral arterial disease (PAD) and healthy controls. In addition, the reproducibility of this method in healthy controls was determined.

\section{Materials and Methods}

Ten PAD patients with intermittent claudication and 10 healthy control subjects were included. Patients underwent contrast-enhanced MR angiography of the peripheral arteries, followed by one DCE MRI examination of the musculature of the calf. Healthy control subjects were examined twice on different days to determine normative values and the interreader and interscan reproducibility of the technique. The MRI protocol comprised dynamic imaging of contrast agent wash-in under reactive hyperemia conditions of the calf musculature. Using pharmacokinetic modeling the hyperemic fractional microvascular blood plasma volume $\left(V_{p}\right.$, unit: \%) of the anterior tibial, gastrocnemius and soleus muscles was calculated.

\section{Results}

$V_{p}$ was significantly lower for all muscle groups in PAD patients $(4.3 \pm 1.6 \%, 5.0 \pm 3.3 \%$ and $6.1 \pm 3.6 \%$ for anterior tibial, gastrocnemius and soleus muscles, respectively) compared to healthy control subjects $(9.1 \pm 2.0 \%, 8.9 \pm 1.9 \%$ and $9.3 \pm 2.1 \%)$. Differences in $V_{p}$ between muscle groups were not significant. The coefficient of variation of $V_{p}$ varied from $10-14 \%$ and $11-16 \%$ at interscan and interreader level, respectively. 


\section{Conclusions}

Using DCE MRI after contrast-enhanced MR angiography with gadofosveset enables reproducible assessment of hyperemic fractional microvascular blood plasma volume of the calf musculature. $V_{p}$ was lower in PAD patients than in healthy controls, which reflects a promising functional (hemodynamic) biomarker for the microvascular impairment of macrovascular lesions. 


\section{Introduction}

Functional measurements in peripheral arterial disease (PAD) are used for diagnostic purposes and for quantifying the hemodynamic consequences of obstructive arterial lesions (1-4). Among these functional measurements, the ankle brachial index (ABI) is the most recognized and widely applied test for diagnosis and therapy monitoring in PAD (1-9). ABI measurements only represent macrovascular functionality, whereas PAD is known to affect the microcirculation as well, particularly in patients with diabetes mellitus $(10,11)$. Functional assessment of the microvascular status of the lower extremities could therefore be a valuable addition to the current diagnostic work-up in PAD to objectively determine disease severity and might be useful in evaluation of therapeutic efficacy in PAD (12-15). MRI is well-suited for comprehensive diagnostic imaging of both macrovascular morphology of the peripheral vascular tree using contrast-enhanced MRA (CE-MRA) (1618 ) and the microvascular functionality by dynamic contrast-enhanced (DCE) MRI $(14,19-21)$.

Commonly used functional measures in DCE MRI are the rate con-

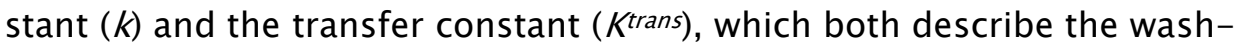
in of a contrast agent. These measures reflect a combination of the rate of microvascular reactivity and permeability, both of which may be significantly impaired in patients with $\operatorname{PAD}(14,19,22,23)$. The rate of vascular reactivity, as determined by rate constant $k$ and transfer constant $K^{\text {trans }}$, however, decreases with age, mainly due to slow progression of vascular resistance as reflected by an increased intima thickness versus luminal diameter ratio (24). Therefore, the clinical relevance of these measures in PAD patients is questionable. In mild PAD, i.e. intermittent claudication, symptoms generally arise and progress slowly during exercise (impaired active hyperemia). Critical ischemia, on the other most severe end of the pathophysiological spectrum, is a chronic condition characterized by hypoperfusion of the distal lower extremity at rest. It is conceivable that in both of these conditions, the absolute degree of microvascular dilatation, rather than its rate, is of more 
clinical relevance and a better reflection of clinical severity of microvascular disease in PAD compared to rate and transfer constants. We hypothesize that maximum microvascular dilatation can be assessed by determining the hyperemic fractional microvascular blood plasma volume $\left(V_{p}\right)$ using DCE MRI. Microvascular blood plasma volume, however, is hard or even impossible to measure using extracellular contrast agents, as clinically applied low-molecular weight (i.e. small-sized) contrast agents extravasate rather rapdily into the interstitial space (22, $25,26)$. Recently introduced blood pool agents are largely prevented from leaking into the interstitial space by a strong, reversible albumin binding and therefore theoretically allow a more reliable assessment of microvascular blood plasma volume with DCE MRI $(27,28)$.

The main purpose of the current study was to describe a method to assess hyperemic microvascular blood plasma volume of the calf musculature and to investigate its ability to discriminate between PAD patients and healthy control subjects using the blood pool contrast agent gadofosveset in dynamic contrast-enhanced (DCE) MRI. Also, the interreader and interscan reproducibility of this method was determined in healthy control subjects.

\section{Materials and Methods}

\section{Study population}

Ten patients (age mean \pm SD: $66.5 \pm 9.9$ years; 8 males and 2 females) with proven PAD (intermittent claudication, Fontaine stage II, (5)), diagnosed by the vascular surgeon, and 10 healthy control subjects without signs and symptoms of PAD (age $24.1 \pm 2.2$ years; 3 males and 7 females) were included in this study. Exclusion criteria were diabetes mellitus, hemodynamic instability, contra-indications for MRI (i.e. claustrophobia, known gadolinium based contrast agent allergy, and estimated glomular filtration rate $<30 \mathrm{~mL} / \mathrm{kg} / 1.73 \mathrm{~m}^{2}$. The institutional medical ethics committee approved the study and all subjects gave written informed consent before inclusion. 


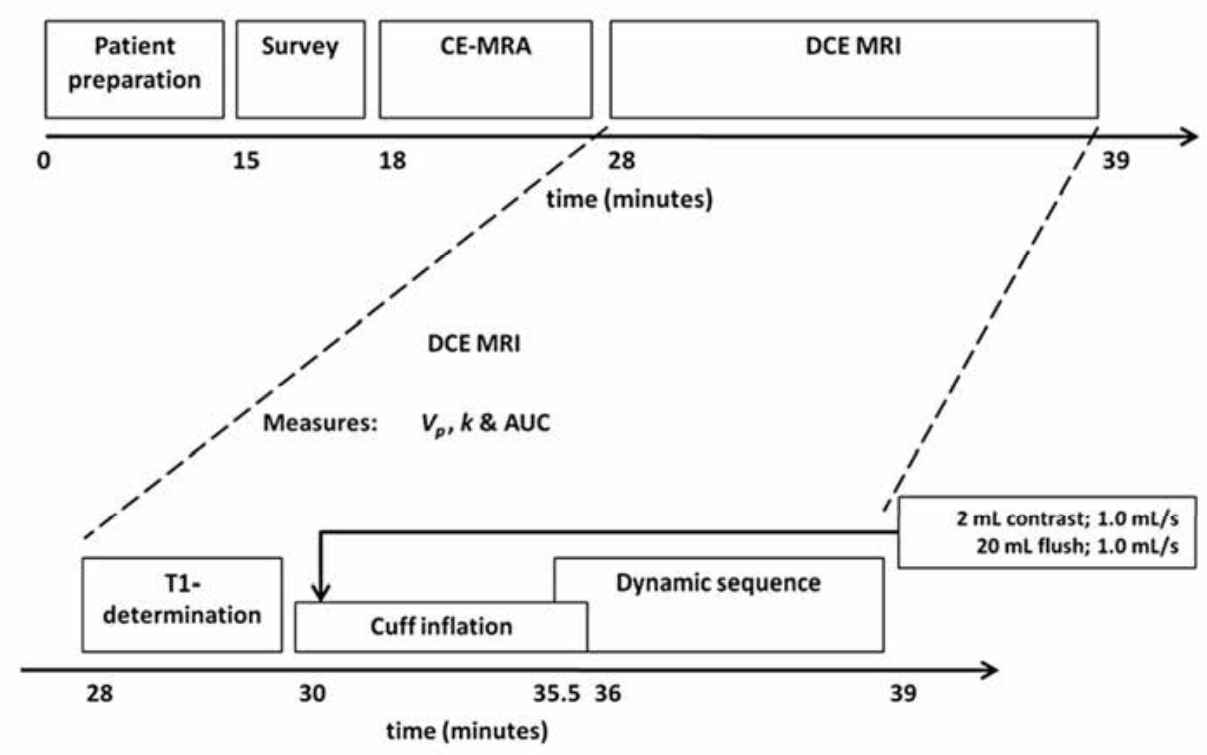

Figure 1. Overview of the imaging protocol. DCE MRI was preceded by conventional threestation contrast-enhanced MR angiography (CE-MRA) for which a dose of $8 \mathrm{~mL}$ gadofosveset was administered. CE-MRA was followed by 'pre-contrast' T1 determination. A 6minute cuff compression of the thigh was used to provoke reactive hyperemia within the calf musculature. Directly after cuff inflation a single dose of $2 \mathrm{~mL}$ gadofosveset was injected. Half a minute before cuff deflation the dynamic contrast-enhanced (DCE) MRI was started.

\section{MRI protocol}

An overview of the imaging protocol is given in figure 1. The entire DCE MRI examination lasted approximately 15 minutes. All scans were performed on a 1.5-T commercially available MRI system (Intera, Philips Medical Systems, Best, The Netherlands). For all acquisitions a dedicated 12-element three-station peripheral vascular phased-array coil (4 elements/station) was used. Subjects were imaged in the supine position and care was taken not to deform calf muscle by calf-bed contact. All subjects were lying in this position for at least 30 minutes before the DCE MRI measurement was started. During this time a conventional three-station contrast enhanced MR angiography (CE-MRA) 
exam was performed in the subject. Therefore, prior to the start of the DCE MRI measurement, all subjects had already received $8 \mathrm{~mL}$ of gadofosveset (Ablavar ${ }^{\circledR}$, Lantheus Medical Imaging, Billerica, MA).

PAD patients were examined once, whereas in healthy control subjects the entire exam was repeated on a different day (interval mean \pm SD: $9.3 \pm 3.5$ days) to determine normative values and the reproducibility of the technique.

Survey. A non-enhanced time-of-flight (TOF) scan of the pelvic, upper and lower leg station was acquired to prescribe the imaging volumes of interest for subsequent morphological and functional imaging. A turbo field echo (TFE) pulse sequence was used with a $180^{\circ}$ inversion prepulse to suppress stationary tissues. Thirty-one transverse slices per station were acquired with 3.3-mm slice thickness and 11-mm interslice gap, and an inferiorly concatenated saturation band. The standard quadrature body coil was used for signal transmission and reception. For positioning of the 3D angiography volumes, maximum intensity projections (MIP) were generated in 3 orthogonal directions.

CE-MRA. A three-station 3D CE-MRA was performed as previously described (29). Prior to contrast medium administration, a nonenhanced 'mask' image data set was acquired with exactly the same acquisition parameters as the CE-MRA, enabling background tissue suppression by image subtraction.

DCE MRI. A dynamic 3D T1-weighted spoiled gradient echo sequence was used for DCE MRI with the following acquisition parameters: TR $8.0 \mathrm{~ms}$, TE $0.91 \mathrm{~ms}$, flip angle $30^{\circ}$, FOV $400 \mathrm{~mm}$, matrix $128 \times 128$, NSA 1 and a parallel imaging (SENSE) reduction factor of 2 (right-left direction). Slice thickness was $6.00 \mathrm{~mm} ; 12$ transverse slices were imaged. Acquired voxel dimensions were $3.13 \times 3.13 \times 6.00 \mathrm{~mm}$. Dynamic scan time was 2.6 seconds. Slices were centered at the maximum diameter of the calf. A series of 80 dynamic scans were acquired in approximately 3.5 minutes. A cuff paradigm was applied to provoke reactive hyperemia $(14,30)$. A fixed dose of $2 \mathrm{~mL}$ gadofosveset was injected directly after cuff inflation, using an automated power injector 


\section{ChAPTER 8}

(Medrad Spectris, Indianola, PA). Cuff inflation was directly followed by contrast injection to allow systemic contrast equilibration in the arterial blood pool during cuff compression and to ensure that DCE MRI data were acquired during the steady state, at which gadofosveset was maximally bound to albumin $(27,28,31)$. The acquisition started 330 seconds after cuff inflation. The cuff was then rapidly deflated at the start of the $6^{\text {th }}$ dynamic scan.

Before the cuff inflation of the dynamic sequence, a series of spoiled fast gradient echo scans with identical contrast and geometry parameters with respect to the dynamic sequence, but with varying flip angles $\left(2,5,10,15,25\right.$ and $\left.35^{\circ}\right)$ were acquired (figure 1). These variable flip angle scans were used for $\mathrm{T} 1$ baseline determination (after contrast agent administration for (E-MRA) and subsequent conversion of signal changes to $\mathrm{T} 1$ relaxation time changes and contrast agent concentration time-courses $(12,32)$.

\section{Image analysis}

Only the most symptomatic leg was analyzed in patients, whereas for healthy control subjects the measures were determined for both legs separately and subsequently averaged. Two independent MRI readers, blinded for each other's results and acquisition order, analyzed the data sets of healthy control subjects. Patient datasets were analyzed by one observer, foreshadowing the good interreader reproducibility we found in healthy control subjects, which were concordant to previous results (33).

The image software application MRIcro (MRIcro, http: / www.mricro.com/) was used to manually draw regions of interest (ROI's) of $3 \times 3$ pixels in the anterior tibial, gastrocnemius and soleus muscles for all 12 slices, resulting in a ROI volume of $6.3 \mathrm{~mL}$. Also, a larger ROI covering the entire cross-section of the calf musculature in four successive slices was drawn. These ROI's were subject to relatively large inter-individual differences in volume due to large variations in calf size in the subjects. An arterial ROI was drawn within the tibiofibular trunk to obtain the arterial input function (AIF). Self-written software 
code (Matlab, The Mathworks inc., Natick, MA) was used for the analysis of $\mathrm{Tl}$-weighted signal intensity time-courses for each ROI. Matlab was also used to calculate the functional microvascular parameters as represented by figure 1 .

The T1-value, prior to the contrast injection of the DCE-MRI but after that of the CE-MRA, were determined from the variable flip angle series for each ROI (34). To calculate the T1-value, the signal curve as a function of the flip angle was fitted to the signal formula for the spoiled fast gradient echo pulse sequence using a non-linear (LevenbergMarquardt) optimization algorithm, which corrects for the inherent nonlinearity in the relation between changes in MRI signal, T1, and contrast agent concentration. Any possible T2* effects were negated by the short TE used. The dynamic sequence was used to calculate the following functional measures: hyperemic fractional microvascular blood plasma volume $\left(V_{p}\right.$, unit: \%), rate constant $\left(k\right.$, unit: $\left.\mathrm{min}^{-1}\right)$ and the area-underthe-curve in the first 90 seconds after contrast arrival (AUC 90 , unit: $\mathrm{mM} \cdot \mathrm{s}$ ). Individual signal time-courses were normalized with respect to resting values, measured prior to contrast agent arrival and converted to contrast agent concentration, using the relation between $\mathrm{Tl}$ relaxation time and the signal intensity for a spoiled gradient echo pulse sequence (12).

The concentration time-course in muscle tissue $C_{t}(t)$ after cuff deflation was empirically modeled by the relation

$$
C_{t}(t)=V_{p} C_{p}^{\max }\left(1-e^{-k t}\right) e^{-m t}
$$

where $V_{p}$ is the fractional hyperemic blood plasma volume in muscle tissue, $C_{p}^{\max }$ is the maximum concentration of contrast agent in blood, $k$ is the rate constant describing the speed of muscle enhancement after cuff deflation, and $m$ represents the tissue clearance rate. The parameters $V_{p}$ and $k$ were calculated by non-linear curve fitting, using a Levenberg-Marquadt optimization algorithm (35). $C_{p}^{\max }$ was determined from the asymptotic concentration level in the femoral artery. The concentration time-courses were used to calculate the area-under-curve for the first 90 seconds after cuff deflation $(A \cup C ; \mathrm{mMs})$. The functional measure 


\section{CHAPTER 8}

$V_{p}$ is related to the degree of (total) microvascular dilatation, whereas the functional measures $k$ and AUC reflect the rate of microvascular reactivity.

\section{Statistical analysis}

A two-samples $t$-test was used to determine the significance of differences in the functional parameters between patients and healthy control subjects. $\mathrm{P}<0.05$ was considered statistically significant. Statistical analysis was performed with commercially available statistical software (SPSS 16.0, SPSS Inc., Chicago, IL).

For healthy control subjects the reproducibility of the measures was calculated, both at interscan and interreader level. Reproducibility of each parameter was expressed as coefficient of variation (CV in \%) and the repeatability coefficient (RC). The CV is derived by dividing overall mean within-subject standard deviation $\left(\mathrm{SD}_{\mathrm{ws}}\right)$ by the mean measurement value over all subjects. The RC is the smallest noticeable difference that can be detected beyond measurement error and is defined as $1.96 \cdot \sqrt{ } 2 \cdot \mathrm{SD}_{\mathrm{ws}}(36,37)$.

Assuming that interscan reproducibility was mainly influenced by scan and time related varariabilities (e.g. variations in slice positioning and day-to-day variations in physiology) it was calculated by averaging results of both MRI readers for each scan (mean of MRI 1 for reader $A$ and $B$ versus the mean of MRI 2 for reader A and B). Conversely, interreader reproducibility is an indicator of the image reading error (e.g. positioning and delineation of ROl's) and is primarily influenced by reader experience. Interreader reproducibility was calculated by averaging the results of both scans for each MRI reader (mean of reader $A$ for MRI 1 and MRI 2 versus the mean of reader B for MRI 1 and MRI 2).

\section{Results}


All included subjects underwent DCE MRI as planned without experiencing side effects or adverse events.

\section{CE-MRA}

CE-MRA revealed stenosis (> 50\%) of the SFA in 6 out of 10 patients, whereas an occlusion of the SFA was found in the remaining 4 patients. In 3 out of 10 patients there were slight vessel wall irregularities in the iliac arteries. The remaining patients had no signs of obstructive lesions in the iliac arteries. At least 2 out of 3 main arteries of the lower leg were free from obstructive lesions in all patients. CE-MRA revealed no signs of PAD in any of the control subjects.

\section{DCE MRI}

Examples of DCE MRI derived time-courses of the entire crosssection of the calf musculature and arterial blood are presented in figure 2. Muscle tissue enhancement was generally less strong in patients compared to healthy control subjects (figure 2). Signal time-courses of the individual muscle groups showed similar results. Strongest enhancement was found in the soleus muscle.

An AIF was obtained for each individual subject within the most prominent arterial structure visible, which was either the tibiofibular trunk or posterior tibial artery in most patients. Quality of the AIF was lower in patients in comparison with the AIF of healthy control subjects. There was a trend $(p=0.07)$ towards higher maximum concentration of the AIF in patients compared to the healthy control subjects. 


\section{CHAPTER 8}

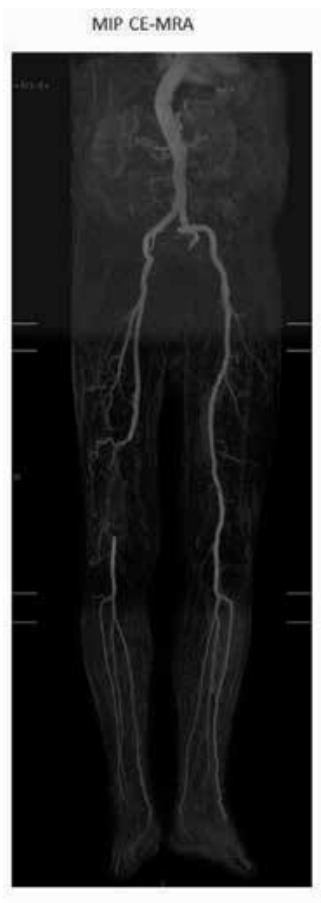

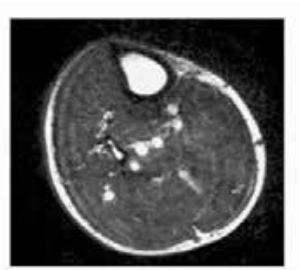
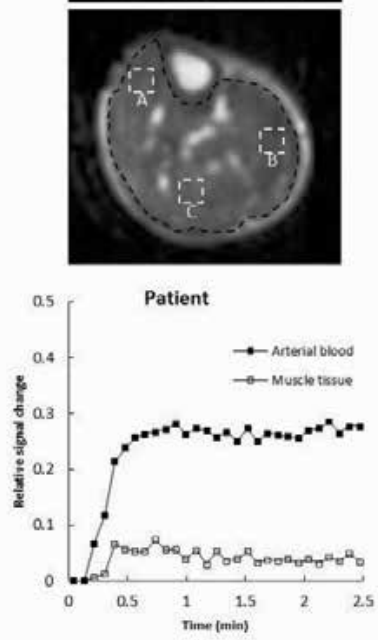

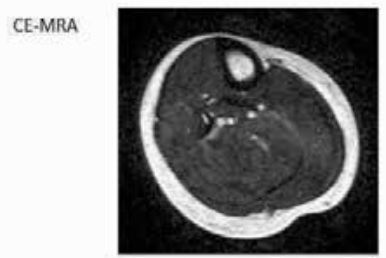

DCE
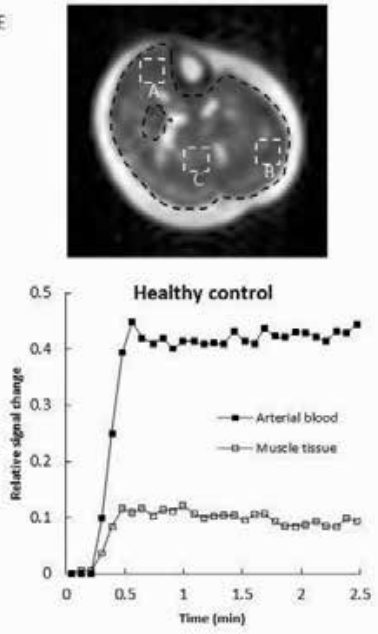

Figure 2. Coronal maximum intensity projection (MIP) of a CE-MRA examination of the peripheral arterial tree of a patient with PAD (left panel) and examples of axial crosssectional CE-MRA and DCE images of a patient with PAD (center panel) and a healthy control subject (right panel), respectively. The comparison for DCE MRI between patients with PAD and healthy control subjects was made for the tibial anterior (A), gastrocnemius (B) and soleus (C) muscle, as well as the entire cross-section of the calf musculature (area within the black dotted lines). The lower center and right panels show representative examples of the relative signal change in blood and the entire cross-section of the calf musculature before and after cuff release in a patient with PAD and healthy control, respectively.

\section{Patients versus healthy control subjects}

Table 1 lists the values of DCE MRI measures obtained in patients and healthy control subjects. $V_{p}$ was significantly lower for all muscle groups in PAD patients compared to healthy control subjects. Differences in $V_{p}$ between patients and controls ranged from $3.2-4.8 \%$ ( $\mathrm{p}<$ 0.01 ). For both patients and controls the differences in $V_{p}$ between 
muscle groups were not significant $(p=0.49$ and $p=0.15$, respectively). $V_{p}$ of the entire cross-section of the calf musculature was significantly higher compared to the $V_{p}$ of individual muscle groups, both for patients and healthy control subjects $(p<0.01)$. The rate constant $k$ was significantly lower in PAD patients for all muscle groups (range differences $5.5-7.9 \mathrm{~min}^{-1}, \mathrm{p}<0.01$ ). The AUC only was significantly lower in PAD patients for the anterior tibial muscle $(\mathrm{p}<0.01) . V_{p}$, $k$ or AUC were not consistently higher or lower in PAD patients with stenosis $(n=6)$ compared to PAD patients with an occlusion $(n=4)$ of the SFA.

\section{Patients Healthy controls}

$(n=10) \quad(n=10)$

\section{Anterior tibial muscle}

$\begin{array}{lccc}V_{p} & \% & 4.3 \pm 1.6 & 9.1 \pm 2.0^{*} \\ K & \text { min }^{-1} & 2.3 \pm 1.6 & 11.2 \pm 5.6^{*} \\ \text { AUC } 90 s & \mathrm{mMs} & 0.35 \pm 0.22 & 0.64 \pm 0.19^{*}\end{array}$

Gastrocnemius muscle

$\begin{array}{lcccc}V_{p} & \% & 5.0 \pm 3.3 & 8.9 \pm 1.9^{*} & \\ K & \mathrm{~min}^{-1} & 4.1 \pm 4.1 & 9.6 \pm 4.0^{*} & \\ \text { AUC } 90 \mathrm{~s} & \mathrm{mMs} & 0.49 \pm 0.26 & 0.55 \pm 0.14 & (\mathrm{p}=0.50)\end{array}$

Soleus muscle

\begin{tabular}{|c|c|c|c|c|}
\hline$V_{p}$ & $\%$ & $6.1 \pm 3.6$ & $9.3 \pm 2.1^{* *}$ & \\
\hline$\kappa$ & $\min ^{-1}$ & $5.1 \pm 3.7$ & $12.3 \pm 4.1^{*}$ & \\
\hline$A U C_{90 s}$ & $\mathrm{mMs}$ & $0.64 \pm 0.28$ & $0.65 \pm 0.15$ & $(p=0.90)$ \\
\hline
\end{tabular}

Cross-section calf musculature

\begin{tabular}{|c|c|c|c|c|}
\hline$V_{p}$ & $\%$ & $7.2 \pm 4.3$ & $12.0 \pm 2.4^{*}$ & \\
\hline$k$ & $\min ^{-1}$ & $5.5 \pm 3.6$ & $10.4 \pm 4.1^{*}$ & \\
\hline$A U C_{90 s}$ & $\mathrm{mMs}$ & $0.78 \pm 0.17$ & $0.87 \pm 0.17$ & $(p=0.18)$ \\
\hline
\end{tabular}

Table 1. DCE MRI of the calf musculature in patients with PAD and healthy control subjects.

Values are presented as mean $\pm \mathrm{SD}$. $\mathrm{V}_{\mathrm{p}}$, fractional microvascular blood plasma volume; $\mathrm{k}$, rate constant; $A_{U} C_{90}$, area under the curve for the first 90 seconds after cuff release.

"p $<0.01,{ }^{* *} p<0.05$ 


\begin{tabular}{|c|c|c|}
\hline & & $\mathrm{T}_{1 \text { pre }}{ }^{*}$ \\
\hline \multirow{4}{*}{$\begin{array}{l}\text { Anterior tibial muscle } \\
\text { Interscan }\end{array}$} & & $688 \pm 29 \mathrm{~ms}$ \\
\hline & & \\
\hline & CV & $3.4 \%$ \\
\hline & $\mathrm{RC}$ & $63 \mathrm{~ms}$ \\
\hline \multicolumn{3}{|l|}{ Interreader } \\
\hline & CV & $1.2 \%$ \\
\hline & $\mathrm{RC}$ & $22 \mathrm{~ms}$ \\
\hline \multirow{4}{*}{$\begin{array}{l}\text { Gastrocnemius muscle } \\
\text { Interscan }\end{array}$} & & $722 \pm 56 \mathrm{~ms}$ \\
\hline & & \\
\hline & $C V$ & $5.4 \%$ \\
\hline & $\mathrm{RC}$ & $108 \mathrm{~ms}$ \\
\hline \multicolumn{3}{|l|}{ Interreader } \\
\hline & $\mathrm{CV}$ & $6.4 \%$ \\
\hline & $\mathrm{RC}$ & $131 \mathrm{~ms}$ \\
\hline \multirow{4}{*}{$\begin{array}{l}\text { Soleus muscle } \\
\text { Interscan }\end{array}$} & & $667 \pm 32 \mathrm{~ms}$ \\
\hline & & \\
\hline & CV & $5.0 \%$ \\
\hline & $\mathrm{RC}$ & $91 \mathrm{~ms}$ \\
\hline \multicolumn{3}{|l|}{ Interreader } \\
\hline & CV & $5.9 \%$ \\
\hline & $\mathrm{RC}$ & $109 \mathrm{~ms}$ \\
\hline \multicolumn{2}{|c|}{ Cross-section calf musculature } & $625 \pm 28 \mathrm{~ms}$ \\
\hline \multicolumn{3}{|c|}{ Interscan } \\
\hline & CV & $4.9 \%$ \\
\hline & $\mathrm{RC}$ & $84 \mathrm{~ms}$ \\
\hline \multicolumn{3}{|l|}{ Interreader } \\
\hline & CV & $2.6 \%$ \\
\hline & $\mathrm{RC}$ & $44 \mathrm{~ms}$ \\
\hline
\end{tabular}

Table 2. Reproducibility of $\mathrm{Tl}$ determination in healthy control subjects

Values are presented as mean $\pm \mathrm{SD}$. $\mathrm{T}_{1 \text { pre }}, \mathrm{T}_{1}$ before administration of contrast agent; CV, coefficient of variation; RC, repeatability coefficient.

* Pre-contrast T1 values were actually obtained after a prior injection of $8 \mathrm{~mL}$ gadofosveset, as used for contrast-enhanced MR angiography of the lower extremities. 


\begin{tabular}{cccc}
\hline & $V_{p}$ & $k$ & AUC $_{\text {90s }}$ \\
\hline Anterior tibial muscle & $9.1 \pm 2.0 \%$ & $11.2 \pm 5.6 \mathrm{~min}^{-1}$ & $0.64 \pm 0.19 \mathrm{mMs}$
\end{tabular}

Interscan

$\begin{array}{cccc}\text { CV } & 12.5 \% & 48.8 \% & 36.5 \% \\ \text { RC } & 3.1 \% & 15.1 \mathrm{~min}^{-1} & 0.64 \mathrm{mMs}\end{array}$

Interreader

\begin{tabular}{rcccc} 
& CV & $13.0 \%$ & $22.8 \%$ & $16.2 \%$ \\
& RC & $3.3 \%$ & $7.1 \mathrm{~min}^{-1}$ & $0.29 \mathrm{mMs}$ \\
\hline Gastrocnemius muscle & & $8.9 \pm 1.9 \%$ & $9.6 \pm 4.0 \mathrm{~min}^{-1}$ & $0.55 \pm 0.14 \mathrm{mMs}$
\end{tabular}
Interscan

$\begin{array}{lccc}\text { CV } & 9.7 \% & 37.8 \% & 25.5 \% \\ \text { RC } & 2.4 \% & 10.0 \mathrm{~min}^{-1} & 0.39 \mathrm{mMs}\end{array}$

Interreader

\begin{tabular}{lcccc} 
& CV & $16.0 \%$ & $22.1 \%$ & $27.3 \%$ \\
& RC & $3.9 \%$ & $5.9 \mathrm{~min}^{-1}$ & $0.41 \mathrm{mMs}$ \\
\hline $\begin{array}{l}\text { Soleus muscle } \\
\text { Interscan }\end{array}$ & & $9.3 \pm 2.1 \%$ & $12.3 \pm 4.1 \mathrm{~min}^{-1}$ & $0.65 \pm 0.15 \mathrm{mMs}$ \\
& & & & \\
& CV & $13.3 \%$ & $19.9 \%$ & $23.3 \%$ \\
& RC & $3.4 \%$ & $6.8 \mathrm{~min}^{-1}$ & $0.42 \mathrm{mMs}$
\end{tabular}

Interreader

\begin{tabular}{lcccc} 
& $\mathrm{CV}$ & $11.2 \%$ & $18.7 \%$ & $12.0 \%$ \\
& $\mathrm{RC}$ & $2.9 \%$ & $6.3 \mathrm{~min}^{-1}$ & $0.22 \mathrm{mMs}$ \\
\hline $\begin{array}{l}\text { Cross-section calf musculature } \\
\text { Interscan }\end{array}$ & $12.0 \pm 2.4 \%$ & $10.4 \pm 4.1 \mathrm{~min}^{-1}$ & $0.87 \pm 0.17 \mathrm{mMs}$ \\
& $\mathrm{CV}$ & $16.3 \%$ & $34.8 \%$ & $20.3 \%$ \\
& $\mathrm{RC}$ & $5.4 \%$ & $9.9 \mathrm{~min}^{-1}$ & $0.49 \mathrm{mMs}$ \\
Interreader & & & \\
& $\mathrm{CV}$ & $2.1 \%$ & $8.4 \%$ & $1.8 \%$ \\
& $\mathrm{RC}$ & $0.7 \%$ & $2.4 \mathrm{~min}^{-1}$ & $0.04 \mathrm{mMs}$ \\
\hline
\end{tabular}

Table 3. Reproducibility of DCE MRI in healthy control subjects.

Values are presented as mean $\pm \mathrm{SD}$. A, maximum concentration; $\mathrm{V}_{\mathrm{p}}$, fractional microvascular blood plasma volume; $k$, rate constant; $A U C_{90 s}$, area under the curve for the first 90 seconds; CV, coefficient of variation; RC, repeatability coefficient. 


\section{Reproducibility}

Values and reproducibility measures of the pre-contrast $\mathrm{Tl}$ assessment in healthy control subjects are given in table $2 . T 1$ assessment showed interscan and interreader CV's below 6 and 7\% respectively.

Reproducibility of the DCE MRI measurements in healthy control subjects is listed in table 3. CV and RC of $V_{p}$ for the different muscle groups were below $17 \%$ and $6 \%$, respectively, at interscan level and below $16 \%$ and $6 \%$, respectively, at interreader level. Interreader and interscan reproducibility of $V_{p}$ were comparable for most muscle groups. Reproducibility values of $V_{p}$ were comparable for the different muscles. Interscan reproducibility of $k$ and AUC showed CV values as high as $49 \%$ and $37 \%$, respectively. Interreader reproducibility was markedly better with a maximum CV of $22 \%$ and $27 \%$ for $k$ and AUC, respectively. Interreader CV was lowest for the entire muscle group ( $8.4 \%$ and $1.8 \%$ for $k$ and AUC, respectively).

\section{Discussion}

In this study we found that gadofosveset, a reversible albumin binding (blood pool) contrast agent, can be used in DCE MRI to reproducibly determine a functional surrogate of the hyperemic microvascular blood plasma volume in the calf. Using this functional measure, the fractional microvascular blood plasma volume $V_{p}$, patients with PAD revealed a reduced $V_{p}$ for all muscle types in the calf relative to healthy controls. Moreover, patients with PAD could be discriminated from healthy subjects on the basis of $V_{p}$. Measures that quantified the rate of vasodilation ( $k$ and AUC) were also reduced in patients with PAD compared to healthy controls. However, interscan and interreader reproducibility of $V_{p}$ were better compared to those of measures that reflect the vasodilation rate ( $k$ and AUC). We also found that functional DCE MRI with a blood pool contrast agent can easily be combined with clinical CE-MRA to assess both macrovascular morphology and microvascular functionality in patients with PAD. 


\section{Pathophysiologic effect in PAD}

The fractional microvascular blood plasma volume $V_{p}$ of the calf musculature in PAD patients with intermittent claudication was significantly lower than in healthy control subjects. A lower $V_{p}$ in patients with PAD can possibly be explained by impaired microvascular dilatative capacity, which is the result of endothelial dysfunction (38-43). This means that DCE MRI can be used to determine the fractional microvascular blood plasma volume of each large muscle group of the calf and as such can identify patients with reduced $V_{p}$ as compared to healthy controls.

$V_{p}$ did show consistent differences between PAD patients with stenosis or occlusions. This can be explained partly by the small sample size (respectively six PAD patients with stenosis and four with occlusion of the SFA), but also by the fact that most of the patients with an occlusion of the SFA revealed well developed collateral arteries at CE-MRA and both patients with stenosis and occlusion had the same clinical symptoms (i.e. intermittent claudication).

There were no significant differences between individual muscle groups within patients or controls. This indicates that this method does not distinguish different muscles of the calf. In healthy control subjects, $V_{p}$ of the entire cross-section of the calf musculature showed significantly higher values when compared to $V_{p}$ of the individual muscles. In patients a similar trend was observed. A higher $V_{p}$ for the entire crosssection of the calf musculature can be explained by the inclusion of relatively large arterial and venous structures (in comparison to $\mathrm{mi}-$ crovessels) in these regions of interest, increasing the average amount of contrast agent within the ROI which results in a higher $V_{p}$. To avoid relatively large blood vessels, assessment of $V_{p}$ of individual muscles is preferred over the $V_{p}$ of the entire cross-section of the calf musculature.

The measures $k$ and the $A U C$ both reflect the initial speed of signal enhancement during reactive hyperemia and were lower in patients compared to healthy controls. This finding indicates lower vascular 


\section{ChAPTER 8}

reactivity in patients with PAD, which is in line with the results of previous studies $(14,19)$.

\section{Reproducibility}

Interscan reproducibility of $V_{p}$ in DCE MRI was good compared to $k$ and the $A U C$. The interscan CV in healthy control subjects of all muscle groups was lower than the relative difference between patients and healthy control subjects. This implies that the reproducibility of the measurement is sufficient to discriminate patients with PAD from healthy controls. The RC for the anterior tibial and gastrocnemius muscles was lower compared to the absolute difference between patients and healthy controls. The RC for the entire cross-section of the calf musculature and the soleus muscle was comparable to the absolute difference between patients and healthy control subjects. A poor RC is probably the result of our small study population and these results might improve with a larger group of subjects.

Reproducibility of $V_{p}$ was in general much higher than the reproducibility of $k$ and the $A U C$, both at interscan and interreader level. This is clearly reflected by the large RC values for $k$ and AUC, which are comparable or even higher than the measured values for $k$ and AUC in healthy control subjects. These findings are in line with those found by Galbraith et al. concerning the extravasation rate constant $K^{\text {trans }}$, using low molecular weight (i.e. small-sized) extracellular contrast agents (19). For a blood pool acting contrast agent, $k$ mainly represents the speed of the filling of the microvasculature in the time period of the measurement, rather than the extravasation of contrast agent into the interstitial space. This rapid filling phase did not prove reproducible and is therefore not suitable as a monitoring measure in clinical practice.

T1 mapping showed good interscan and interreader reproducibility of $\mathrm{T} 1$ values for each muscle group. High interreader reproducibility indicates that the technique is relative insensitive for the exact location at which a ROI is drawn within a muscle group and that the technique is highly reader-independent. This is especially desirable for monitoring purposes, as in clinical routine it is likely that follow-up examinations 
will be assessed by different readers. Good reproducibility of $\mathrm{Tl}$ mapping suggests that our scan and analysis method is reliable and that the moderate reproducibility we found for $V_{p}$ is the result of biologic factors and/or the cuff paradigm.

\section{Clinical relevance}

Functional assessment of muscle perfusion of the lower extremities with dynamic contrast-enhanced (DCE) MRI, could be a valuable addition to the diagnostic work-up in PAD to objectively determine disease severity. An added advantage is that DCE MRI can be combined with the standard morphological assessment of PAD with CE-MRA. Also, DCE-MRI could be of value in non-invasive assessment of the efficacy of novel therapeutic strategies such as stem cell and gene therapy. DCE MRI of calf musculature may indicate the contribution of macrovascular blood flow on muscle perfusion and provide an objective diagnostic tool to determine the influence and severity of microvascular dysfunction in $\operatorname{PAD}(13-15,19)$.

Commonly used extracellular contrast agents rapidly extravasate into the interstitial space and are therefore less suitable to determine biomarkers of the microvascular blood plasma volume (25).

\section{Study considerations}

Although we found large differences between patients with PAD and healthy control subjects for $V_{p}$ and the reproducibility of $V_{p}$ was good, the number of subjects in this study is relatively small and further research will be needed to confirm our results by blinded analysis in more patients and compare them with age and gender matched control subjects. The observed differences between patients with PAD and the controls is expectedly smaller for age-matched, thus older, controls. In addition, it would be clinically relevant to include patients with different symptoms of PAD to assess variations in the involvement of microvascular impairment in PAD. 


\section{ChAPTER 8}

In all subjects a three-station CE-MRA of the peripheral arteries was performed, prior to the DCE MRI. For CE-MRA the first bolus injection of gadofosveset was used. This means all subjects already had gadofosveset in their systemic circulation at the start of the DCE MRI. The residual presence of contrast agent in the circulation affects the T1 determination and its variability. However, a combination of both exams is highly desirable for clinical practice and our results prove the possibility to discriminate patients from control subjects and provided good reproducibility of $V_{p}$ (and $\mathrm{T} 1$ ), despite the previous bolus injection for angiography.

Although in reality a small fraction $( \pm 15 \%)$ of unbound gadosfosveset is available for extravasation into the interstitial space, the influence of this fraction upon the outcome can be neglected as the relative relaxivity of albumin-bound gadofosveset is much higher compared to the unbound fraction ( $r_{1}$ of respectively 19 and $5.2 \mathrm{~L} \cdot \mathrm{mmol}^{-1} \cdot \mathrm{s}^{-1}$ in blood plasma at $37^{\circ} \mathrm{C}$ at $1.5 \mathrm{~T}$ ) and the total fraction of albumin-bond gadofosveset $( \pm 85 \%)$ is much larger as compared to the unbound fraction $(27,28,31)$. In this study, gadofosveset proved to be a usable contrast agent for DCE MRI of muscle tissue in PAD to obtain a surrogate functional measure of microvascular blood plasma volume in patients with PAD.

\section{Conclusion}

DCE MRI after CE-MRA using a blood pool contrast agent is able to determine the hyperemic microvascular blood plasma volume $V_{p}$ of the calf musculature and to identify patients with PAD as compared to healthy control subjects. In addition, the reproducibility of $V_{p}$ was good in healthy control subjects. Together with the ability to acquire DCE MRI data combined with CE-MRA during a single examination makes DCE MRI with a blood pool acting agent a valuable addition to CE-MRA to assess both the macrovascular morphology and functional (hemodynamic) status of the calf microvasculature in patients with PAD. 


\section{References}

1. Hirsch AT, Haskal ZJ, Hertzer NR, et al. ACC/AHA 2005 Practice Guidelines for the management of patients with peripheral arterial disease (lower extremity, renal, mesenteric, and abdominal aortic): a collaborative report from the American Association for Vascular Surgery/Society for Vascular Surgery, Society for Cardiovascular Angiography and Interventions, Society for Vascular Medicine and Biology, Society of Interventional Radiology, and the ACC/AHA Task Force on Practice Guidelines (Writing Committee to Develop Guidelines for the Management of Patients With Peripheral Arterial Disease): endorsed by the American Association of Cardiovascular and Pulmonary Rehabilitation; National Heart, Lung, and Blood Institute; Society for Vascular Nursing; TransAtlantic Inter-Society Consensus; and Vascular Disease Foundation. Circulation. 2006;113(11):e463-654.

2. Norgren L, Hiatt WR, Dormandy JA, et al. Inter-Society Consensus for the Management of Peripheral Arterial Disease (TASC II). J Vasc Surg. 2007;45 Suppl S:S5-67.

3. Gerhard-Herman M, Gardin JM, Jaff M, et al. Guidelines for noninvasive vascular laboratory testing: a report from the American Society of Echocardiography and the Society for Vascular Medicine and Biology. Vasc Med. 2006;11(3):183-200.

4. Creager MA. Clinical assessment of the patient with claudication: the role of the vascular laboratory. Vasc Med. 1997;2(3):231-7.

5. Aslam F, Haque A, Foody J, et al. Peripheral arterial disease: current perspectives and new trends in management. South Med J. 2009;102(11):1141-9.

6. McDermott MM, Criqui $\mathrm{MH}$, Greenland $\mathrm{P}$, et al. Leg strength in peripheral arterial disease: associations with disease severity and lower-extremity performance. J Vasc Surg. 2004;39(3):523-30.

7. McDermott MM, Greenland P, Liu K, et al. Leg symptoms in peripheral arterial disease: associated clinical characteristics and functional impairment. JAMA. $2001 ; 286(13)$ :1599-606.

8. Resnick HE, Lindsay RS, McDermott MM, et al. Relationship of high and low ankle brachial index to all-cause and cardiovascular disease mortality: the Strong Heart Study. Circulation. 2004;109(6):733-9.

9. Begelman SM, Jaff MR. Noninvasive diagnostic strategies for peripheral arterial disease. Cleve Clin J Med. 2006;73 Suppl 4:S22-9. 


\section{CHAPTER 8}

10. Chao CY, Cheing GL. Microvascular dysfunction in diabetic foot disease and ulceration. Diabetes Metab Res Rev. 2009;25(7):604-14.

11. Wong WT, Wong SL, Tian XY, et al. Endothelial dysfunction: the common consequence in diabetes and hypertension. J Cardiovasc Pharmacol. 2010;55(4):300-7.

12. de Lussanet QG, van Golde JC, Beets-Tan RG, et al. Dynamic contrastenhanced MRI of muscle perfusion combined with MR angiography of collateral artery growth in a femoral artery ligation model. NMR Biomed. 2007;20(8):717-25.

13. Isbell DC, Epstein $\mathrm{FH}$, Zhong $\mathrm{X}$, et al. Calf muscle perfusion at peak exercise in peripheral arterial disease: measurement by first-pass contrast-enhanced magnetic resonance imaging. J Magn Reson Imaging. 2007;25(5): 1013-20.

14. Thompson RB, Aviles RJ, Faranesh AZ, et al. Measurement of skeletal muscle perfusion during postischemic reactive hyperemia using contrastenhanced MRI with a step-input function. Magn Reson Med. 2005;54(2):289-98.

15. Weber MA, Krix M, Delorme S. Quantitative evaluation of muscle perfusion with CEUS and with MR. Eur Radiol. 2007;17(10):2663-74.

16. Nelemans PJ, Leiner T, de Vet HC, et al. Peripheral arterial disease: metaanalysis of the diagnostic performance of MR angiography. Radiology. 2000;217(1):105-14.

17. Koelemay MJ, Lijmer JG, Stoker J, et al. Magnetic resonance angiography for the evaluation of lower extremity arterial disease: a meta-analysis. JAMA. 2001;285(10):1338-45.

18. Menke J, Larsen J. Meta-analysis: Accuracy of contrast-enhanced magnetic resonance angiography for assessing steno-occlusions in peripheral arterial disease. Ann Intern Med. 2010;153(5):325-34.

19. Galbraith SM, Lodge MA, Taylor NJ, et al. Reproducibility of dynamic contrast-enhanced $\mathrm{MRI}$ in human muscle and tumours: comparison of quantitative and semi-quantitative analysis. NMR Biomed. 2002;15(2):132-42.

20. de Vries M, de Koning PJ, de Haan MW, et al. Accuracy of semiautomated analysis of 3D contrast-enhanced magnetic resonance angiography for detection and quantification of aortoiliac stenoses. Invest Radiol. $2005 ; 40(8): 495-503$. 
21. Leiner T, Kessels AG, Nelemans PJ, et al. Peripheral arterial disease: comparison of color duplex US and contrast-enhanced MR angiography for diagnosis. Radiology. 2005;235(2):699-708.

22. Tofts PS, Brix G, Buckley DL, et al. Estimating kinetic parameters from dynamic contrast-enhanced T(1)-weighted MRI of a diffusable tracer: standardized quantities and symbols. J Magn Reson Imaging. 1999;10(3):223-32.

23. Kershaw LE, Buckley DL. Precision in measurements of perfusion and microvascular permeability with T1-weighted dynamic contrast-enhanced MRI. Magn Reson Med. 2006;56(5):986-92.

24. Mitchell GF. Effects of central arterial aging on the structure and function of the peripheral vasculature: implications for end-organ damage. J Appl Physiol. 2008;105(5):1652-60.

25. Tofts PS. Modeling tracer kinetics in dynamic Gd-DTPA MR imaging. J Magn Reson Imaging. 1997;7(1):91-101.

26. Tofts PS, Berkowitz BA. Measurement of capillary permeability from the Gd enhancement curve: a comparison of bolus and constant infusion injection methods. Magn Reson Imaging. 1994;12(1):81-91.

27. Caravan P, Cloutier NJ, Greenfield MT, et al. The interaction of MS-325 with human serum albumin and its effect on proton relaxation rates. J Am Chem Soc. 2002;124(12):3152-62.

28. Caravan P, Parigi G, Chasse JM, et al. Albumin binding, relaxivity, and water exchange kinetics of the diastereoisomers of MS-325, a gadolinium(III)-based magnetic resonance angiography contrast agent. Inorg Chem. 2007;46(16):6632-9.

29. de Vries M, Nijenhuis RJ, Hoogeveen RM, et al. Contrast-enhanced peripheral MR angiography using SENSE in multiple stations: feasibility study. J Magn Reson Imaging. 2005;21(1):37-45.

30. Ledermann HP, Schulte AC, Heidecker HG, et al. Blood oxygenation leveldependent magnetic resonance imaging of the skeletal muscle in patients with peripheral arterial occlusive disease. Circulation. 2006;113(25):2929-35.

31. Rohrer M, Bauer H, Mintorovitch J, et al. Comparison of magnetic properties of MRI contrast media solutions at different magnetic field strengths. Invest Radiol. 2005;40(11):715-24. 


\section{CHAPTER 8}

32. Haacke M, Brown R, Thompson M, et al. T1 estimation from SSI measurements at multiple flip angles. In: Haacke M, Brown R, Thompson M, et al., eds. Magnetic resonance imaging: physical principles ans sequence design. New York, NY: John Willey \& Sons, Inc; 1999:654-661.

33. Versluis B, Backes WH, van Eupen MG, et al. Magnetic resonance imaging in peripheral arterial disease: reproducibility of the assessment of morphological and functional vascular status. Invest Radiol. 2011;46(1):1124.

34. Haacke EM, Filleti CL, Gattu R, et al. New algorithm for quantifying vascular changes in dynamic contrast-enhanced MRI independent of absolute T1 values. Magn Reson Med. 2007;58(3):463-72.

35. Press $\mathbf{W H}$, Numerical recipes in C: the art of scientific computing. 2nd ed. 1992, Cambridge; New York: Cambridge University Press. xxvi, 994 p.

36. Bland JM, Altman DG. Measurement error. BMJ. 1996;312(7047): 1654.

37. Jansen JF, Kooi ME, Kessels AG, et al. Reproducibility of quantitative cerebral T2 relaxometry, diffusion tensor imaging, and $1 \mathrm{H}$ magnetic resonance spectroscopy at 3.0 Tesla. Invest Radiol. 2007;42(6):327-37.

38. Grover-Paez F, Zavalza-Gomez AB. Endothelial dysfunction and cardiovascular risk factors. Diabetes Res Clin Pract. 2009;84(1):1-10.

39. Libby P, Ridker PM, Hansson GK. Inflammation in atherosclerosis: from pathophysiology to practice. J Am Coll Cardiol. 2009;54(23):2129-38.

40. Rocha VZ, Libby P. Obesity, inflammation, and atherosclerosis. Nat Rev Cardiol. 2009;6(6):399-409.

41. Ribeiro F, Alves AJ, Teixeira M, et al. Endothelial function and atherosclerosis: circulatory markers with clinical usefulness. Rev Port Cardiol. 2009;28(10):1121-51.

42. Vita JA, Hamburg NM. Does endothelial dysfunction contribute to the clinical status of patients with peripheral arterial disease? Can J Cardiol. 2010;26 Suppl A:45A-50A.

43. Ziegler MA, Distasi MR, Bills RG, et al. Marvels, mysteries, and misconceptions of vascular compensation to peripheral artery occlusion. Microcirculation. 2010;17(1):3-20. 


\section{CHAPTER 9}

General discussion 
Chapter 9 
Peripheral arterial disease (PAD) is an expression of generalized arteriosclerosis, an inflammatory disease of the vessel wall of arteries, caused by well-known noxious stimuli like smoking, diabetes mellitus, hypertension, dyslipidemia and hyperhomocysteinemia $(1,2)$. Despite progress in cardiovascular risk management and treatment, peripheral arterial disease (PAD) remains a major health care problem among the elderly, affecting up to $20 \%$ of the worldwide population of 70 years and older $(1,2)$. Clinical symptoms associated with PAD range from intermittent claudication to critical ischemia, and are the result of compromised arterial flow towards the lower extremities due to obstructive arterial lesions at the macrovascular level and/or microvascular disease (1).

Collateral artery formation is one of the potential physiological mechanisms by which the human body attempts to compensate for the ischemia induced by these obstructive arterial lesions (3). However, collateral formation is a complicated and incompletely understood process, thought to involve both adaptation of existing arteries and formation of novel collateral arteries.

Early imaging of PAD might be beneficial to determine a reliable starting point for patient monitoring and the best therapeutic options. In addition, imaging is potentially useful to monitor the effects of novel vessel-stimulating therapies, to increase our knowledge concerning these processes and perhaps to help in the early identification of possible non-responders to therapy. MRI is a highly attractive imaging modality for this purpose because it is virtually the only clinically available modality that can be used to reliably assess vascular morphology as well as function in a single examination.

This thesis addresses the development and clinical utility of morphologic and functional vascular MRI in patients with PAD. The main objectives were to assess the suitability of quantitative MRI techniques in diagnosis and longitudinal follow up of patients with peripheral arterial disease. Different morphologic and functional vascular MRI techniques were tested for reproducibility and discriminative capability, and based on these results improvements to existing techniques are proposed to better differentiate between diseased and healthy vascula- 


\section{CHAPTER 9}

ture. Also, a standardized approach to acquire quantitative morphologic and functional vascular information was developed. The results of this thesis are placed in a broader context for which the role of MRI is related to other diagnostic (imaging) tools in PAD.

\section{Current diagnostic work-up of patients with PAD}

PAD is foremost a clinical diagnosis, based upon the symptoms of patients. The typical patient history and physical examination are often sufficient to diagnose PAD and to grade the severity of disease, according to the Rutherford and Fontaine classification (1, 2, 4-6). History taking should be focused on the presence of risk factors, relation between physical activity and complaints, pain free and/or maximum walking distance and presence or absence of rest pain. Physical examination should encompass a comparison between the affected and unaffected extremity as far as color changes, hair loss, toenails (thickened and brittle toenails may appear in patients with PAD), muscular volume (affected skeletal muscle groups may show atrophy), venous filling time and temperature differences are concerned. Patients with PAD often exhibit diminished or absent arterial pulsation as well as bruits during palpation in the presence of a stenosis $(6,7)$. Abnormal femoral pulsations have proven to be highly specific and offer a high positive predictive value for the presence of PAD, although the sensitivity is low (8).

Because many patients with PAD are asymptomatic, history and physical examination alone are not entirely reliable for detection of PAD $(1,6,9,10)$. However, a variety of diagnostic tests are available for early diagnosis in asymptomatic subjects and objective confirmation of the diagnosis in symptomatic patients with suspected PAD. One of the most widely used means of objectively documenting the presence and severity of PAD is measurement of the ankle-brachial index ( $A B I)$. $A B I$ is a fast, widely available and cost-effective test for this purpose. Sensitivity of $A B I$ measurements for diagnosing $P A D$ is high and the $A B I$ can be used to determine the severity and progression of PAD over time (13, 15-17). Determination of $A B I$ is also suitable as a screening tool in the high-risk, 
asymptomatic population to confirm the presence of suspected PAD ( 1 , $18,19)$. If the $A B I$ at rest is normal, repeating the measurement after a treadmill test might further increase the sensitivity, as a systolic pressure gradient might arise after exercise in patients with hemodynamically significant stenoses.

Despite the clear advantages there are also drawbacks to $A B I$ measurement. There are several well-known limitations such as relatively poor interobserver variability and inability to reliably acquire the $A B I$ in many patients with stiff and uncompressible ankle arteries due to severe arterial wall calcifications (20-22). In this latter category of patients, $A B I$ may be incongruently high. In fact, an abnormally high $A B I$ $(>1.4)$ has also been associated with the presence of PAD. Studies have shown that the $A B I$ cannot be (reliably) measured in at least

$5-10 \%$ of the diabetic patients, as these patients are most prone to develop severely calcified artery walls, especially regarding small arteries, such as at the level of the ankle and the pedal arteries $(14,17,22-$ 27).

Once the presence of PAD is confirmed, the next step in the routine diagnostic workup of patients with PAD is to determine the exact location and extent of obstructive arterial lesions. Segmental pressure measurements are most often used for this purpose. Plethysmographic cuffs placed over the brachial arteries and at different distinct locations around the lower extremity are used to measure systolic blood pressure with a Doppler device, comparable to $A B I$ measurements $(6,28)$. In general, a gradient difference of more than $20 \mathrm{mmHg}$ between two adjacent levels is considered indicative for the presence of hemodynamically significant obstructive arterial lesions.

Often, segmental pressure measurements are combined with pulse volume recordings. The combination of segmental systolic pressures and pulse volume recordings results in a diagnostic accuracy of $97 \%$ (30, 31 ). The technique of segmental pressure measurements is very comparable to $A B I$ measurements. Not surprisingly, these measurements suffer from the same limitations related to uncompressible arteries. Moreover, pulse waveform measurements are a subjective (qualitative) rather than 


\section{ChAPTER 9}

an objective (quantitative) functional measurement, with limitations in heavily calcified arteries. Pulse waveform measurements are less accurate than duplex ultrasonography for localizing lesions (28).

Segmental pressure measurements are often followed by duplex ultrasonography (DUS). DUS combines Doppler waveform analysis and Doppler velocities. DUS is mainly used for determining the exact location of the obstructive arterial lesion and for differentiating between stenosis and occlusion. A normal arterial flow waveform is triphasic, which in the presence of obstructive arterial lesions will become either bi- or monophasic. An increase of more than $100 \%$ of the peak systolic velocity relative to the proximal normal arterial segment indicates the presence of significant arterial lesions. DUS is used to determine the degree of stenosis, which is done by a combination of flow waveform analysis and the peak systolic velocity $(28,32)$. Besides diagnostic purposes and treatment planning, DUS is also used for the follow-up of patients with (treated) PAD.

DUS is an accurate non-invasive test to confirm the presence of PAD. However, it requires the technical skill of an experienced vascular technologist and the accuracy is operator and patient dependent (32). A complete duplex examination of both legs is rather time consuming (over 40 minutes). Also, the accuracy for the aortoiliac vessels is limited, amongst others due to body habitus and bowel gas. The sensitivity and specificity for the detection of hemodynamically significant arterial stenosis are lower than for CT angiography (CTA) or MR angiography (MRA) $(32,33)$. In line with other ultrasonographic techniques, DUS is unable to measure flow within heavily calcified arteries and the accuracy of the technique diminishes when there are multiple obstructive arterial lesions within close proximity (28). Although DUS is relatively inexpensive, in up to $43 \%$ of the patients there is need for additional imaging according to the DIPAD trial (34), which in the end results in significantly higher costs for DUS when compared to CT angiography and comparable costs when compared with MR angiography. Also, it has been proven that if patients do have a preference for either MRA or DUS in the diagnostic work-up for PAD, MRA is preferred over DUS (35). 


\section{Imaging of PAD}

None of the above mentioned diagnostic tests provide adequate morphologic information. Although functional information suits the purpose of diagnosing and monitoring, vascular morphology is required to determine the location and severity of stenosis and plays an important role in choosing the appropriate therapy (i.e. conservative management, percutaneous intervention or surgery). Imaging of the peripheral arteries in PAD is preserved for patients being considered for invasive therapy only in order to localize arterial lesions and to plan the correct approach of the intervention $(1,18,36)$. Radiological imaging techniques comprise invasive digital subtraction angiography (DSA), which is considered the 'gold standard' for the diagnosis of PAD, and non-invasive CTA and MRA. The introduction of CTA and MRA has made it possible to obtain highly accurate morphologic data on the macrovascular morphologic vascular status in PAD patients and to assess the suitability of arterial lesions for intervention, without the need for invasive angiography (37-39). The rapid development of multidetector CT (MDCT) technology has motivated the clinical use of CTA to examine the peripheral vasculature, and allows fast visualization of the complete peripheral arterial tree with high accuracy. The overall sensitivity and specificity for the assessment of significant arterial stenosis are 95-97\% and $91-98 \%$, respectively (40). Major advantages of CTA are high spatial and contrast resolution and fast acquisition times, whereas the important disadvantages of CTA are the potentially harmful contrast agents and radiation. Furthermore, CTA has limited diagnostic accuracy in (heavily) calcified arteries when compared to DSA and MRA (32).

Over the last decade contrast-enhanced MRA (CE-MRA) has established itself as a highly accurate non-invasive diagnostic imaging method for the morphological evaluation of the large conduit arteries in PAD patients. Sensitivity and specificity of CE-MRA are comparable with invasive intra-arterial digital subtraction angiography (DSA) $(33,38,41)$. In chapter 2 of this thesis we have demonstrated that CE-MRA is an easy and reproducible method to quantify arteries with a detection limit of 


\section{ChAPTER 9}

$0.5 \mathrm{~mm}$ and to assess the diameter of the collateral re-entrance zone in the upper leg (42).

Recently introduced blood pool contrast agents, which reversely bind with blood plasma albumin molecules, thereby limiting extravasation of contrast agent into the interstitial space, increase the temporal imaging window for CE-MRA. This allows high-resolution CE-MRA acquisitions and whole-body CE-MRA examinations to be performed with even better diagnostic accuracy (43-47). MRA has important advantages over DSA and CTA. MRA is a non-invasive imaging modality as compared to DSA and there is no need for iodinated contrast agents nor usage of ionizing radiation in MRA. Compared to CTA, the ability of MRA for stent assessment is generally poor. On the other hand, MRA is better suited to accurately visualize the arterial lumen of heavily calcified arteries when compared to CTA (32).

DSA, CTA and MRA are all suitable to accurately depict vascular morphology in patients with PAD. Morphologic images, however, provide little or no information on the hemodynamic consequences of obstructive vascular lesions in PAD patients, whereas above mentioned functional tests provide little or no morphologic information. To supplement morphologic information, it is therefore highly desirable to have quantitative non-invasive imaging tools available, that can be easily combined with existing morphologic imaging tools. MR imaging has the potential to overcome these limitations and can facilitate a variety of functional measurements at both the macro- and microvascular level in patients with PAD, as is shown in this thesis. The hemodynamic significance of obstructive arterial lesions can be determined, as well as the impact of vascular adaptations resulting from different treatment strategies. Combined morphologic and functional methods to visualize and quantify the vascular status in PAD patients are useful in several ways. For example to confirm the diagnosis and to grade the severity of PAD. However, morphological and functional data are also suitable for patient follow-up and therapy monitoring, for studying the mechanisms underlying vascular adaptations, such as collateral artery 
formation, and for studying the effect of established and novel therapies aimed at ameliorating PAD.

\section{Functional MRI}

MRI enables comprehensive diagnostic imaging of both the anatomy of the peripheral vascular tree as well as functionality in terms of macrovascular flow and surrogate parameters of tissue perfusion and oxygenation (48-54). In this thesis the reproducibility, discriminative capability and clinical usage of different MRI techniques able to depict macro- and microvascular function were evaluated. Macrovascular function was assessed by measuring arterial peak flow (APF) in the popliteal artery with quantitative $2 \mathrm{D}$ cine MR phase contrast imaging (PCI) $(50,55-58)$. Microvascular function was determined by perfusion measurements in calf musculature using dynamic contrast-enhanced (DCE) MRI $(49,53,59)$, as well as the oxygenation measurements using dynamic blood oxygen level-dependent (BOLD) MRI (48, 52, 60-64).

Arterial peak flow (APF), defined as the maximum systolic flow, is an attractive arterial flow measure that can be obtained with PCI measurements. APF measurements have the advantage of being a fast MRI technique, with acquisition times well below one minute, which can easily be added to existing MRA protocols. In other words, in patients scheduled for MRA both morphologic and functional vascular status can be assessed at the same time $(42,65-68)$. Reproducibility of APF is high, as was shown in chapter 2 and 6 of this thesis $(42,68)$. The APF is an important determinant of the systolic blood pressure (69), that exhibits large differences between PAD patients and control subjects, which has been shown in chapter 2,4 and $6(42,68)$. In chapter 4 we also demonstrated that the APF is able to differentiate between patients with intermittent claudication and critical ischemia, with high reliability, which is comparable with ABI measurements. However, APF measurements could be determined in up to $91 \%$ of 183 PAD patients, as opposed to a success rate of just $71 \%$ for $A B I$ measurements. Although quantitative flow measurements can also be used to measure mean flow and peak velocity, the results of chapter 6 of this thesis indicate that 


\section{CHAPTER 9}

reproducibility and discriminative capability are best for peak flow (50, 68).

DCE MRI is an example of a functional MRI test to assess microvascular function. DCE MRI is able to determine regional blood perfusion in skeletal muscle tissue, and therefore measures the influence of the presence of peripheral arterial disease at end-organ level $(59,70)$. By dynamically recording enhancement of muscle tissue after injection of contrast medium and concurrently measuring arterial signal, quantitative information on the microcirculation can be obtained. When a small molecular contrast agent (e.g. gadopentate dimeglumine) is used for DCE-MRI the wash-in rate of the tissue is relatively fast and primarily determined by microvascular flow. Using a two-compartment pharmacokinetic model that relates arterial concentration to the tissue concentration, the dynamic transfer of the contrast agent from the blood space to the tissue can be quantified in terms of the unidirectional influx $K_{i}$ (unit: $\min ^{-1}$ ), which is a surrogate quantity for microvascular flow.

Unfortunately, skeletal muscle perfusion is highly variable and relatively low under resting conditions and does not represent perfusion during physical exertion $(53,71)$. To mimic the vascular response to an active muscle use in a standardized way, a cuff inflation-deflation paradigm can be used to provoke reactive hyperemia (72). Besides provoking reactive hyperemia, the cuff paradigm also results in a stepped arterial input function of contrast agent concentration $(53,72)$.

Although DCE MRI has proven to be a promising technique in highly perfused organs, such as brain and kidney tissue, the results of chapter 2 in this thesis indicate that perfusion of the calf musculature cannot be determined reproducibly with DCE MRI (42). Reproducibility studies in skeletal muscle are unfortunately scarce, but our work corroborates to prior DCE MRI studies in various tumor types and skeletal muscle performed under resting conditions, which also reported unfavorable reproducibility measures, particularly for muscle tissue $(49,53)$. In the study by Galbraith et al. flow in resting state muscle was low and variable. Large day-to-day variations in endothelial function due to variations in endothelial permeability and bioavailability of nitric oxide 
might explain these results $(49,73)$. Although DCE-MRI allows very interesting insights into vascular (patho)physiology, the results of chapter 2 indicate a lack of discriminatory power for DCE MRI, as the differences in $K_{i}$ between patients with PAD and healthy controls were not significant.

In contrast to DCE MRI, BOLD MRI is a non-contrast enhanced functional MRI technique to measure perfusion. The BOLD phenomenon is based on local susceptibility changes due to changes in blood oxygenation and BOLD imaging is sensitive to the concentration of paramagnetic deoxyhemoglobin (endogenous contrast). Its presence in blood results in a mismatch between the magnetic susceptibility of tissue and blood and thereby shortening of the $\mathrm{T}_{2}{ }^{*}$ of blood and its immediate surroundings $(52,60)$. The disproportionally high inflow of oxygenated blood during postischemic reactive hyperemia, results in an initially rapidly increasing $T_{2}{ }^{*}$, as the concentration of deoxyhemoglobin will decrease and the mismatch is reduced $(52,60,61,74-78)$.

As is the case with DCE MRI, BOLD techniques have shown promising results in tissues with a high perfusion rate, such as brain and tumor tissue. However, the results in chapter 2 of this thesis indicate poor reproducibility for BOLD imaging as far as calf muscle perfusion is concerned. Besides, there was no significant difference between patients with PAD and healthy controls in this study (42).

In summary, we demonstrated that macrovascular flow can be reproducibly determined by MRI, whereas methods used to measure microvascular function were subject to large variations. Our observation of poor microvascular reproducibility is remarkable given the reproducibility of the APF at the level of the popliteal artery (i.e. blood supply to calf muscle). There are two likely underlying reasons for this apparent discrepancy. First, the microcirculation of the calf muscle itself is likely more prone to day-to-day variations than macrovascular flow as the microcirculation and endothelial function are influenced by many intrinsic (i.e. hormonal and circadian cycles, insulin supply, shunting and rerouting of blood) and extrinsic (i.e. outside temperature, season, time of the day, food, pharmacologic) factors (79). Many of these factors are 


\section{CHAPTER 9}

susceptible to day-to-day variations and will therefore inevitably negatively impact reproducibility of microvascular function. A second factor that might partly explain our findings is that microvascular function was assessed under reactive hyperemic conditions, whereas flow was measured under resting conditions.

\section{New developments}

The results of this thesis indicate, that currently APF is the most promising MR derived functional vascular measurement for use in clinical practice. APF measurements are much more reproducible and are best able to identify patients with PAD compared to other functional vascular MRI measurements evaluated in this thesis. Besides, APF has the major advantage that it is a fast MRI technique, with acquisition times well below one minute, which can easily be added to existing MRA-protocols, so that for those patients scheduled for MRA both morphologic and functional vascular status can be interrogated at the same time $(42,65-68)$. This may be especially useful, when other techniques, such as $A B I$ measurements, have failed. APF measurements might eventually even serve as a replacement for other functional measurements, at least in patients with an indication for MRA.

However, one of the strengths of other functional tests, especially $A B I$ measurements, is a high degree of standardization. The $A B I$ is measured at the same location using the same technique in every patient. Such a standardized protocol is a necessity for a functional measurement in order to be suitable for longitudinal follow-up or therapy monitoring in patients and will further increase the discriminative capability of a technique. As APF measurements are relatively new and not widely accepted, currently no such standardized protocol does exist for APF measurements, nor are there normative values known for APF measurements. Although APF measurements are relatively fast, it would be desirable to have a single, predefined location to measure the APF in all patients with PAD, at which the ability to differentiate between a diseased and non-diseased vasculature is highest. In chapter 4 of this thesis we demonstrated a standardized approach in which the APF was 
measured in the proximal common femoral artery, proximal superficial femoral artery and the popliteal artery in patients with PAD, independent from the location of obstructive arterial lesions, and in non-PAD patients. The results of this study indicate that measurement of APF at the level of the popliteal artery shows the largest difference between PAD and non-PAD patients, best discriminative capability and highest success rates of the measurement as compared to the APF acquired at the level of the common femoral artery and superficial femoral artery, even though differences with the superficial femoral artery are small. The popliteal artery therefore seems to be the most suitable predefined location for a standardized measurement of the APF in PAD patients. Although APF measurements at the level of the popliteal artery can be used to differentiate between patients with and without PAD, there remains an overlap in APF values between patients and healthy subjects, as can be seen in the histograms of chapter 4. Especially in PAD patients with intermittent claudication blood flow at rest might be - as expected - near-normal depending on the severity of the obstructive lesion, or recovers fast to near-normal values after exercise due to collateral artery formation (80-87). These (near-)normal resting flow values explain the absence of clinical symptoms at rest in intermittent claudication. Symptoms in these PAD patients, however, characteristically appear during physical exercise, when the flow demands towards the lower extremity increases. In an attempt to increase the sensitivity of APF measurements in these patients, we therefore developed a method to measure the flow reserve and tested its ability to discriminate patients with intermittent claudication from healthy control subjects (chapter 6).

The results of this study revealed that flow reserve is severely compromised in patients with PAD compared to healthy controls. This indicates that flow reserve therefore is able to determine the hemodynamic consequences of obstructive arterial lesions in PAD patients and to discriminate between patients with intermittent claudication and healthy controls. So, the concept of blood flow reserve, originally introduced by Coffman and Gregg in 1960 for the coronary arteries (88), also seems to apply to the peripheral circulation $(83,84,89-91)$. Lower flow 


\section{ChAPTER 9}

reserve in the popliteal artery of PAD patients can be explained by the presence of obstructive arterial lesions, resulting in a decrease of the pressure gradient distal to the vascular lesion and thereby a decrease of the maximum hyperemic flow distal to these lesions (91).

Although the APF already provides useful functional information on the hemodynamic significance of obstructive arterial lesions in intermittent claudication, by measuring the flow reserve, we were able to identify more patients with PAD compared to resting APF measurements alone. These initial results also indicate that for suspected PAD patients with (near-)normal resting APF values, assessment of flow reserve might provide important additional functional information with respect to the hemodynamic significance of the arterial lesions, allowing better differentiation between diseased and non-diseased vasculature. Further studies in larger populations will be needed to determine the true additional value of flow reserve in the assessment of the hemodynamic significance of obstructive arterial lesions, both in patients with intermittent claudication and more severe stages of PAD.

Microvascular function as measured with conventional DCE MRI is poorly reproducible and has limited discriminative capability. Nevertheless, microvascular function remains an interesting phenomenon to study, as PAD is known to affect the microcirculation as well, particularly in patients with diabetes mellitus(92, 93). Functional assessment of microvascular status of the lower extremities could therefore be a valuable addition to the current diagnostic work-up in PAD to objectively determine disease severity and might be useful in evaluation of therapeutic efficacy in $\operatorname{PAD}(53,59,70,94)$. Commonly used functional measures in DCE MRI, the rate constant $(k)$ and the transfer constant ( $K^{\text {trans }}$ ), reflect a combination of the rate of microvascular reactivity and permeability, both of which may be significantly impaired in patients with PAD (49, 53, 95, 96). The rate of vascular reactivity, however, decreases with age, mainly due to slow progression of vascular resistance as reflected by an increased intima thickness versus luminal diameter ratio (97). Therefore, the clinical relevance of these measures in PAD patients is questionable. In mild PAD, i.e. intermittent claudica- 
tion, symptoms generally arise and progress slowly during exercise (i.e. impaired active hyperemia). Critical ischemia, on the other, most severe end of the pathophysiological spectrum, is a chronic condition characterized by hypoperfusion of the distal lower extremity at rest. It is conceivable that in both of these conditions, the absolute degree of microvascular dilatation, rather than its rate, is of more clinical relevance and a better reflection of clinical severity of microvascular disease in PAD compared to both the rate and transfer constant. In chapter 7 of this thesis we therefore hypothesized that DCE MRI may better be used to measure the maximum microvascular dilatation by determining the hyperemic fractional microvascular blood plasma volume $\left(V_{p}\right)$. Microvascular blood plasma volume, however, is hard or even impossible to measure using extracellular contrast agents, as clinically applied lowmolecular weight (i.e. small-sized) contrast agents extravasate rather rapidly into the interstitial space $(95,98,99)$. Blood pool contrast agents, however, are largely prevented from leaking into the interstitial space by a strong, reversible albumin binding and therefore theoretically allow a more reliable assessment of microvascular blood plasma volume with DCE MRI $(100,101)$. The results of this study indeed indicated that DCE MRI using a blood pool contrast agent is able to determine the hyperemic microvascular blood plasma volume $V_{p}$ of the calf musculature and to identify patients with PAD as compared to healthy control subjects. In addition, the reproducibility of $V_{p}$ was better as compared to conventional DCE MRI. Together with the ability to acquire DCE MRI data combined with CE-MRA during a single examination makes DCE MRI with a blood pool contrast agent a valuable addition to CE-MRA to assess both the macrovascular morphology and functional (hemodynamic) status of the calf microvasculature in patients with PAD. Especially in diabetic patients we expect this method to be of value, although this remains to be investigated.

\section{Future developments}

The results of this thesis indicate that MRI is capable of assessing both vascular morphology and vascular function in the same examina- 


\section{CHAPTER 9}

tion. The APF is a fast and easy to use MRI technique, that can be added to existing MRA-protocols. In its current state, APF measurements may already be useful in PAD patients, when other techniques, such as $A B I$ measurements, have failed. APF measurements, however, might eventually even serve as a replacement for other functional measurements.

Although imaging in PAD is currently only applied in those patients who are considered for invasive therapy, it can be argued whether or not this is the most cost-effective approach in the near future. In the present-day situation, the most suitable interventional approach in PAD patients is defined in most cases only after a patient has undergone physical examination, $A B I$ measurement, segmental pressure measurement, DUS and either MRA or CTA. This is a rather time consuming, patient unfriendly and costly approach. As many patients with PAD end up undergoing MRA anyway, early MRA with additional functional MRI may be a much more patient friendly and, at the end, cost-effective approach in the diagnostic work-up of PAD patients. Especially as vascular morphology and function can be assessed with a single MRI examination, while together taking less time than for example a full DUS examination. Results of the DIPAD trial already indicate that early imaging in PAD may be a more cost-effective approach than the current diagnostic work-up in PAD patients (34).

Nevertheless, before functional vascular MRI, and especially APF measurements, are capable of replacing existing functional measurements, more research is needed. As far as APF measurements are concerned, normative values need to be determined in large groups of PAD and non-PAD subjects. Besides, the influence of APF measurements or the correlation between APF and clinical outcome of PAD patients will have to be evaluated to determine the true clinical contribution of APF measurements in the diagnostic work-up of PAD patients in comparison with traditional functional measurements. Also, the cost-effectiveness of early MRA with additional functional measurements has to be evaluated. With these data available, MRA may play a much more prominent role in the diagnostic work-up of PAD patients in the near future. 
Morphologic and functional vascular imaging in patients with PAD may in the future also play a role of importance in elucidating the biological mechanisms with which the human body compensates for local ischemia and to monitor the effects of established and novel vessel-stimulating therapies, to increase our knowledge concerning these processes and to perhaps help in the early identification of possible non-responders to therapy. In chapter 5 of this thesis we used MRA and APF measurements to evaluate the effect of supervised exercise therapy upon vascular status in PAD patients with intermittent claudication. Even though MRI in this study was not able to detect macrovascular changes, these imaging techniques can still be useful for the monitoring and follow-up of patients with PAD treated with other, new therapeutic options aiming to improve vascularization, such as with stem cells and endothelial growth factors (i.e. vascular endothelial growth factor (VEGF) and basic fibroblast growth factor (bFGF)), which are currently being developed for treatment of both intermittent claudication and critical ischemia (102-105). 


\section{References}

1. Hirsch AT, Haskal ZJ, Hertzer NR, et al. ACC/AHA 2005 guidelines for the management of patients with peripheral arterial disease (lower extremity, renal, mesenteric, and abdominal aortic): executive summary a collaborative report from the American Association for Vascular Surgery/Society for Vascular Surgery, Society for Cardiovascular Angiography and Interventions, Society for Vascular Medicine and Biology, Society of Interventional Radiology, and the ACC/AHA Task Force on Practice Guidelines (Writing Committee to Develop Guidelines for the Management of Patients With Peripheral Arterial Disease) endorsed by the American Association of Cardiovascular and Pulmonary Rehabilitation; National Heart, Lung, and Blood Institute; Society for Vascular Nursing; TransAtlantic Inter-Society Consensus; and Vascular Disease Foundation. J Am Coll Cardiol. 2006;47(6): 1239-312.

2. Norgren L, Hiatt WR, Dormandy JA, et al. Inter-Society Consensus for the Management of Peripheral Arterial Disease (TASC II). Eur J Vasc Endovasc Surg. 2007;33 Suppl 1:S1-75.

3. Stewart KJ, Hiatt WR, Regensteiner JG, et al. Exercise training for claudication. N Engl J Med. 2002;347(24):1941-51.

4. Rutherford RB, Baker JD, Ernst C, et al. Recommended standards for reports dealing with lower extremity ischemia: revised version. J Vasc Surg. 1997;26(3):517-38.

5. McDermott MM, Greenland P, Liu K, et al. Leg symptoms in peripheral arterial disease: associated clinical characteristics and functional impairment. JAMA. 2001;286(13):1599-606.

6. Aslam F, Haque A, Foody J, et al. Peripheral arterial disease: current perspectives and new trends in management. South Med J. 2009;102(11):1141-9.

7. McGee SR,Boyko EJ. Physical examination and chronic lower-extremity ischemia: a critical review. Arch Intern Med. 1998;158(12):1357-64.

8. Criqui $\mathrm{MH}$, Fronek $\mathrm{A}$, Klauber MR, et al. The sensitivity, specificity, and predictive value of traditional clinical evaluation of peripheral arterial disease: results from noninvasive testing in a defined population. Circulation. 1985;71(3):516-22.

9. Ouriel K. Peripheral arterial disease. Lancet. 2001;358(9289):1257-64. 
10. Ouriel K. Detection of peripheral arterial disease in primary care. JAMA. 2001;286(11):1380-1.

11. McDermott $M M$, Criqui $M H$, Greenland $P$, et al. Leg strength in peripheral arterial disease: associations with disease severity and lower-extremity performance. J Vasc Surg. 2004;39(3):523-30.

12. Resnick HE, Lindsay RS, McDermott MM, et al. Relationship of high and low ankle brachial index to all-cause and cardiovascular disease mortality: the Strong Heart Study. Circulation. 2004;109(6):733-9.

13. Doobay AV, Anand SS. Sensitivity and specificity of the ankle-brachial index to predict future cardiovascular outcomes: a systematic review. Arterioscler Thromb Vasc Biol. 2005;25(7):1463-9.

14. Potier L, Abi Khalil C, Mohammedi K, et al. Use and Utility of Ankle Brachial Index in Patients with Diabetes. Eur J Vasc Endovasc Surg. 2010.

15. Dachun X, Jue L, Liling Z, et al. Sensitivity and specificity of the ankle-brachial index to diagnose peripheral artery disease: a structured review. Vasc Med. 2010;15(5):361-9.

16. Guo X, Li J, Pang W, et al. Sensitivity and specificity of ankle-brachial index for detecting angiographic stenosis of peripheral arteries. Circ J. 2008;72(4):605-10.

17. Aboyans V, Ho E, Denenberg JO, et al. The association between elevated ankle systolic pressures and peripheral occlusive arterial disease in diabetic and nondiabetic subjects. J Vasc Surg. 2008;48(5):1197-203.

18. Norgren L, Hiatt WR, Dormandy JA, et al. Inter-Society Consensus for the Management of Peripheral Arterial Disease (TASC II). J Vasc Surg. 2007;45 Suppl S:S5-67.

19. Hirsch AT, Criqui MH, Treat-Jacobson D, et al. Peripheral arterial disease detection, awareness, and treatment in primary care. JAMA. 2001;286(1 1):1317-24.

20. van Langen H, van Gurp J, Rubbens L. Interobserver variability of anklebrachial index measurements at rest and post exercise in patients with intermittent claudication. Vasc Med. 2009;14(3):221-6.

21. Allen J, Oates CP, Henderson J, et al. Comparison of lower limb arterial assessments using color-duplex ultrasound and ankle/brachial pressure index measurements. Angiology. 1996;47(3):225-32.

22. Stein R, Hriljac I, Halperin JL, et al. Limitation of the resting ankle-brachial index in symptomatic patients with peripheral arterial disease. Vasc Med. 2006;11(1):29-33. 


\section{CHAPTER 9}

23. Brooks B, Dean R, Patel S, et al. TBI or not TBI: that is the question. Is it better to measure toe pressure than ankle pressure in diabetic patients? Diabet Med. 2001;18(7):528-32.

24. Giachelli CM. Vascular calcification mechanisms. J Am Soc Nephrol. 2004;15(12):2959-64.

25. Goss DE, de Trafford J, Roberts VC, et al. Raised ankle/brachial pressure index in insulin-treated diabetic patients. Diabet Med. 1989;6(7):576-8.

26. London GM, Guerin AP, Marchais SJ, et al. Arterial media calcification in end-stage renal disease: impact on all-cause and cardiovascular mortality. Nephrol Dial Transplant. 2003;18(9):1731-40.

27. Raines JK, Darling RC, Buth J, et al. Vascular laboratory criteria for the management of peripheral vascular disease of the lower extremities. Surgery. 1976;79(1):21-9.

28. Begelman SM, Jaff MR. Noninvasive diagnostic strategies for peripheral arterial disease. Cleve Clin J Med. 2006;73 Suppl 4:S22-9.

29. Bonham P. Measuring toe pressures using a portable photoplethysmograph to detect arterial disease in high-risk patients: an overview of the literature. Ostomy Wound Manage. 2011;57(11):36-44.

30. Rutherford RB, Lowenstein DH, Klein MF. Combining segmental systolic pressures and plethysmography to diagnose arterial occlusive disease of the legs. Am J Surg. 1979;138(2):211-8.

31. Darling RC, Raines JK, Brener BJ, et al. Quantitative segmental pulse volume recorder: a clinical tool. Surgery. 1972;72(6):873-7.

32. Owen AR, Roditi GH. Peripheral arterial disease: the evolving role of noninvasive imaging. Postgrad Med J. 2011;87(1025):189-98.

33. Leiner $\mathrm{T}$, Kessels AG, Nelemans PJ, et al. Peripheral arterial disease: comparison of color duplex US and contrast-enhanced MR angiography for diagnosis. Radiology. 2005;235(2):699-708.

34. Ouwendijk R, de Vries M, Stijnen T, et al. Multicenter randomized controlled trial of the costs and effects of noninvasive diagnostic imaging in patients with peripheral arterial disease: the DIPAD trial. AJR Am J Roentgenol. 2008;190(5):1349-57.

35. Visser K, Bosch JL, Leiner T, et al. Patients' preferences for MR angiography and duplex US in the work-up of peripheral arterial disease. Eur J Vasc Endovasc Surg. 2003;26(5):537-43.

36. Rofsky NM,Adelman MA. MR angiography in the evaluation of atherosclerotic peripheral vascular disease. Radiology. 2000;214(2):325-38. 
37. Leiner $\mathrm{T}$, Tordoir $\mathrm{JH}$, Kessels AG, et al. Comparison of treatment plans for peripheral arterial disease made with multi-station contrast mediumenhanced magnetic resonance angiography and duplex ultrasound scanning. J Vasc Surg. 2003;37(6):1255-62.

38. de Vries M, de Koning PJ, de Haan MW, et al. Accuracy of semiautomated analysis of 3D contrast-enhanced magnetic resonance angiography for detection and quantification of aortoiliac stenoses. Invest Radiol. 2005;40(8):495-503.

39. Jakobs TF, Wintersperger BJ, Becker CR. MDCT-imaging of peripheral arterial disease. Semin Ultrasound CT MR. 2004;25(2):145-55.

40. Met R, Bipat S, Legemate DA, et al. Diagnostic performance of computed tomography angiography in peripheral arterial disease: a systematic review and meta-analysis. JAMA. 2009;301(4):415-24.

41. de Vries M, Nijenhuis RJ, Hoogeveen RM, et al. Contrast-enhanced peripheral MR angiography using SENSE in multiple stations: feasibility study. J Magn Reson Imaging. 2005;21(1):37-45.

42. Versluis B, Backes WH, van Eupen MG, et al. Magnetic resonance imaging in peripheral arterial disease: reproducibility of the assessment of morphological and functional vascular status. Invest Radiol. 2011;46(1):1124.

43. Giovagnoni A, Catalano C. Application of blood-pool agents in visualization of peripheral vessels. Eur Radiol. 2007;1 7 Suppl 2:B18-23.

44. Kos S, Reisinger C, Aschwanden $M$, et al. Pedal angiography in peripheral arterial occlusive disease: first-pass i.v. contrast-enhanced MR angiography with blood pool contrast medium versus intraarterial digital subtraction angiography. AJR Am J Roentgenol. 2009;192(3):775-84.

45. Hadizadeh DR, Gieseke J, Lohmaier SH, et al. Peripheral MR angiography with blood pool contrast agent: prospective intraindividual comparative study of high-spatial-resolution steady-state MR angiography versus standard-resolution first-pass MR angiography and DSA. Radiology. 2008;249(2):701-11.

46. Grijalba FU, Esandi MC. Comparison of gadofosveset-enhanced threedimensional magnetic resonance angiography with digital subtraction angiography for lower-extremity peripheral arterial occlusive disease. Acta Radiol. 2010;51(3):284-9. 


\section{CHAPTER 9}

47. Nielsen YW, Eiberg JP, Logager VB, et al. Whole-body magnetic resonance angiography with additional steady-state acquisition of the infragenicular arteries in patients with peripheral arterial disease. Cardiovasc Intervent Radiol. 2010;33(3):484-91.

48. Baudelet C, Cron GO, Gallez B. Determination of the maturity and functionality of tumor vasculature by MRI: correlation between BOLD-MRI and DCE-MRI using P792 in experimental fibrosarcoma tumors. Magn Reson Med. 2006;56(5):1041-9.

49. Galbraith SM, Lodge MA, Taylor NJ, et al. Reproducibility of dynamic contrast-enhanced $\mathrm{MRI}$ in human muscle and tumours: comparison of quantitative and semi-quantitative analysis. NMR Biomed. 2002;15(2):132-42.

50. Mohajer K, Zhang H, Gurell D, et al. Superficial femoral artery occlusive disease severity correlates with MR cine phase-contrast flow measurements. J Magn Reson Imaging. 2006;23(3):355-60.

51. Carlier PG, Bertoldi D, Baligand C, et al. Muscle blood flow and oxygenation measured by NMR imaging and spectroscopy. NMR Biomed. 2006;19(7):954-67.

52. Ledermann HP, Schulte AC, Heidecker HG, et al. Blood oxygenation leveldependent magnetic resonance imaging of the skeletal muscle in patients with peripheral arterial occlusive disease. Circulation. 2006;113(25):2929-35.

53. Thompson RB, Aviles RJ, Faranesh AZ, et al. Measurement of skeletal muscle perfusion during postischemic reactive hyperemia using contrastenhanced MRI with a step-input function. Magn Reson Med. 2005;54(2):289-98.

54. Lebon V, Brillault-Salvat C, Bloch G, et al. Evidence of muscle BOLD effect revealed by simultaneous interleaved gradient-echo NMRI and myoglobin NMRS during leg ischemia. Magn Reson Med. 1998;40(4):551-8.

55. Meyer RA, Foley JM, Harkema SJ, et al. Magnetic resonance measurement of blood flow in peripheral vessels after acute exercise. Magn Reson Imaging. 1993;11(8):1085-92.

56. Bakker CJ, Kouwenhoven M, Hartkamp MJ, et al. Accuracy and precision of time-averaged flow as measured by nontriggered 2D phase-contrast MR angiography, a phantom evaluation. Magn Reson Imaging. 1995;13(7):959-65. 
57. Klein WM, Bartels LW, Bax L, et al. Magnetic resonance imaging measurement of blood volume flow in peripheral arteries in healthy subjects. J Vasc Surg. 2003;38(5):1060-6.

58. Prakash A, Garg R, Marcus EN, et al. Faster flow quantification using sensitivity encoding for velocity-encoded cine magnetic resonance imaging: in vitro and in vivo validation. J Magn Reson Imaging. 2006;24(3):676-82.

59. Isbell DC, Epstein FH, Zhong X, et al. Calf muscle perfusion at peak exercise in peripheral arterial disease: measurement by first-pass contrast-enhanced magnetic resonance imaging. J Magn Reson Imaging. 2007;25(5):1013-20.

60. Barrett T, Brechbiel M, Bernardo M, et al. MRI of tumor angiogenesis. J Magn Reson Imaging. 2007;26(2):235-49.

61. Noseworthy MD, Bulte DP,Alfonsi J. BOLD magnetic resonance imaging of skeletal muscle. Semin Musculoskelet Radiol. 2003;7(4):307-15.

62. Duteil S, Wary C, Raynaud JS, et al. Influence of vascular filling and perfusion on BOLD contrast during reactive hyperemia in human skeletal muscle. Magn Reson Med. 2006;55(2):450-4.

63. Stainsby JA, Wright GA. Monitoring blood oxygen state in muscle microcirculation with transverse relaxation. Magn Reson Med. 2001;45(4):66272.

64. Bulte DP, Alfonsi J, Bells S, et al. Vasomodulation of skeletal muscle BOLD signal. J Magn Reson Imaging. 2006;24(4):886-90.

65. Dumoulin CL. Phase contrast MR angiography techniques. Magn Reson Imaging Clin N Am. 1995;3(3):399-411.

66. Walker MF, Souza SP, Dumoulin CL. Quantitative flow measurement in phase contrast MR angiography. J Comput Assist Tomogr. 1988;12(2):304-13.

67. Bakker CJ, Hoogeveen RM, Viergever MA. Construction of a protocol for measuring blood flow by two-dimensional phase-contrast MRA. J Magn Reson Imaging. 1999;9(1):119-27.

68. Versluis B, Dremmen MH, Nelemans PJ, et al. MRI of arterial flow reserve in patients with intermittent claudication: feasibility and initial experience. PLoS One. 2012;7(3):e31514.

69. Bishara RA, Taha W, Alfarouk MO, et al. Duplex detected ankle peak systolic velocity: a new parameter for the assessment of degree of peripheralischemia. Int Angiol. 2004;23(4):368-72. 


\section{CHAPTER 9}

70. Weber MA, Krix M, Delorme S. Quantitative evaluation of muscle perfusion with CEUS and with MR. Eur Radiol. 2007;17(10):2663-74.

71. Wu WC, Wang J, Detre JA, et al. Hyperemic flow heterogeneity within the calf, foot, and forearm measured with continuous arterial spin labeling MRI. Am J Physiol Heart Circ Physiol. 2008;294(5):H2129-36.

72. Patel PP, Koppenhafer SL, Scholz TD. Measurement of kinetic perfusion parameters of gadoteridol in intact myocardium: effects of ischemia/reperfusion and coronary vasodilation. Magn Reson Imaging. 1995;13(6):799-806.

73. Ziegler MA, Distasi MR, Bills RG, et al. Marvels, mysteries, and misconceptions of vascular compensation to peripheral artery occlusion. Microcirculation. 2010;17(1):3-20.

74. Noseworthy MD, Kim JK, Stainsby JA, et al. Tracking oxygen effects on MR signal in blood and skeletal muscle during hyperoxia exposure. J Magn Reson Imaging. 1999;9(6):814-20.

75. Prielmeier F, Merboldt KD, Hanicke W, et al. Dynamic high-resolution MR imaging of brain deoxygenation during transient anoxia in the anesthetized rat. J Cereb Blood Flow Metab. 1993;13(5):889-94.

76. Prielmeier F, Nagatomo Y, Frahm J. Cerebral blood oxygenation in rat brain during hypoxic hypoxia. Quantitative MRI of effective transverse relaxation rates. Magn Reson Med. 1994;31(6):678-81.

77. Donahue KM, Van Kylen J, Guven S, et al. Simultaneous gradientecho/spin-echo EPI of graded ischemia in human skeletal muscle. J Magn Reson Imaging. 1998;8(5):1106-13.

78. Jordan BF, Kimpalou JZ, Beghein N, et al. Contribution of oxygenation to BOLD contrast in exercising muscle. Magn Reson Med. 2004;52(2):391-6.

79. Deanfield J, Donald A, Ferri C, et al. Endothelial function and dysfunction. Part I: Methodological issues for assessment in the different vascular beds: a statement by the Working Group on Endothelin and Endothelial Factors of the European Society of Hypertension. J Hypertens. 2005;23(1):7-17.

80. Kinney TB, Rose SC. Intraarterial pressure measurements during angiographic evaluation of peripheral vascular disease: techniques, interpretation, applications, and limitations. AJR Am J Roentgenol. 1996;166(2):277-84.

81. Mathien GM, Terjung RL. Muscle blood flow in trained rats with peripheral arterial insufficiency. Am J Physiol. 1990;258(3 Pt 2):H759-65. 
82. Brevetti LS, Paek R, Brady SE, et al. Exercise-induced hyperemia unmasks regional blood flow deficit in experimental hindlimb ischemia. J Surg Res. 2001;98(1):21-6.

83. Corcoran HA, Smith BE, Mathers $\mathrm{P}$, et al. Laser Doppler imaging of reactive hyperemia exposes blood flow deficits in a rat model of experimental limb ischemia. J Cardiovasc Pharmacol. 2009;53(6):446-51.

84. Bragadeesh T, Sari I, Pascotto M, et al. Detection of peripheral vascular stenosis by assessing skeletal muscle flow reserve. J Am Coll Cardiol. 2005;45(5):780-5.

85. May AG, Van De Berg L, Deweese JA, et al. Critical arterial stenosis. Surgery. 1963;54:250-9.

86. Pena CS, McCauley TR, Price TB, et al. Quantitative blood flow measurements with cine phase-contrast MR imaging of subjects at rest and after exercise to assess peripheral vascular disease. AJR Am J Roentgenol. 1996;167(1):153-7.

87. Hudlicka O, Brown MD. Adaptation of skeletal muscle microvasculature to increased or decreased blood flow: role of shear stress, nitric oxide and vascular endothelial growth factor. J Vasc Res. 2009;46(5):504-12.

88. Coffman JD, Gregg DE. Reactive hyperemia characteristics of the myocardium. Am J Physiol. 1960;199:1 143-9.

89. Lindner JR, Womack L, Barrett EJ, et al. Limb stress-rest perfusion imaging with contrast ultrasound for the assessment of peripheral arterial disease severity. JACC Cardiovasc Imaging. 2008;1 (3):343-50.

90. Hoffman Jl. Maximal coronary flow and the concept of coronary vascular reserve. Circulation. 1984;70(2):153-9.

91. Galderisi M, D'Errico A. Beta-blockers and coronary flow reserve: the importance of a vasodilatory action. Drugs. 2008;68(5):579-90.

92. Chao CY, Cheing GL. Microvascular dysfunction in diabetic foot disease and ulceration. Diabetes Metab Res Rev. 2009;25(7):604-14.

93. Wong WT, Wong SL, Tian XY, et al. Endothelial dysfunction: the common consequence in diabetes and hypertension. J Cardiovasc Pharmacol. $2010 ; 55(4): 300-7$.

94. de Lussanet QG, van Golde JC, Beets-Tan RG, et al. Dynamic contrastenhanced MRI of muscle perfusion combined with MR angiography of collateral artery growth in a femoral artery ligation model. NMR Biomed. 2007;20(8):717-25. 


\section{CHAPTER 9}

95. Tofts PS, Brix G, Buckley DL, et al. Estimating kinetic parameters from dynamic contrast-enhanced $\mathrm{T}(1)$-weighted MRI of a diffusable tracer: standardized quantities and symbols. J Magn Reson Imaging. 1999;10(3):223-32.

96. Kershaw LE, Buckley DL. Precision in measurements of perfusion and microvascular permeability with T1-weighted dynamic contrast-enhanced MRI. Magn Reson Med. 2006;56(5):986-92.

97. Mitchell GF. Effects of central arterial aging on the structure and function of the peripheral vasculature: implications for end-organ damage. J Appl Physiol. 2008;105(5):1652-60.

98. Tofts PS. Modeling tracer kinetics in dynamic Gd-DTPA MR imaging. J Magn Reson Imaging. 1997;7(1):91-101.

99. Tofts PS, Berkowitz BA. Measurement of capillary permeability from the Gd enhancement curve: a comparison of bolus and constant infusion injection methods. Magn Reson Imaging. 1994;12(1):81-91.

100. Caravan P, Cloutier NJ, Greenfield MT, et al. The interaction of MS-325 with human serum albumin and its effect on proton relaxation rates. J Am Chem Soc. 2002;124(12):3152-62.

101. Caravan P, Parigi G, Chasse JM, et al. Albumin binding, relaxivity, and water exchange kinetics of the diastereoisomers of MS-325, a gadolinium(III)-based magnetic resonance angiography contrast agent. Inorg Chem. 2007;46(16):6632-9.

102. Lawall H, Bramlage P, Amann B. Treatment of peripheral arterial disease using stem and progenitor cell therapy. J Vasc Surg. 2010.

103. Lawall H, Bramlage P, Amann B. Stem cell and progenitor cell therapy in peripheral artery disease. A critical appraisal. Thromb Haemost. 2010;103(4):696-709.

104. Lederman RJ, Mendelsohn FO, Anderson RD, et al. Therapeutic angiogenesis with recombinant fibroblast growth factor-2 for intermittent claudication (the TRAFFIC study): a randomised trial. Lancet. 2002;359(9323):2053-8.

105. Rajagopalan S, Mohler ER, 3rd, Lederman RJ, et al. Regional angiogenesis with vascular endothelial growth factor in peripheral arterial disease: a phase II randomized, double-blind, controlled study of adenoviral delivery of vascular endothelial growth factor 121 in patients with disabling intermittent claudication. Circulation. 2003;108(16):1933-8. 


\section{APPENDIX}

Summary / samenvatting

Dankwoord

Curriculum vitae

List of publications 
APPENDIX 
Summary 
APPENDIX 
Peripheral arterial disease (PAD) is an expression of generalized arteriosclerosis resulting in compromised arterial flow to the lower extremities due to obstructive peripheral arterial lesions. Symptoms range from intermittent claudication, commonly defined as pain and / or cramping of the calf musculature in response to exercise, relieved by a short period of rest, to critical limb ischemia, defined as ischemic rest pain, ulceration or gangrene.

Arterial lesions can present at both the macro vascular level (i.e. lesions in large conduit arteries), and the micro vascular level, in which small arteries, arterioles and capillaries are affected. Microvascular disease is most pronounced in the subgroup of PAD patients with diabetic mellitus, whereas macrovascular disease is the major cause of symptoms in non-diabetic PAD patients.

Collateral artery formation is one of the mechanisms by which the human body can compensate for the ischemia resulting from these obstructive arterial lesions. However, collateral formation is a complicated process, thought to involve both adaptation of existing arteries and the formation of novel collateral arteries, and to date the conditions under which collateral formation takes place in PAD are still poorly understood.

Currently, the diagnosis of PAD is typically made on the basis of the typical clinical history, palpation of pedal pulsations and by measurement of the ankle-brachial index ( $\mathrm{ABI})$. Consequently, imaging of the peripheral vascular tree is only preserved for those patients being considered for invasive therapy with the aim to localize arterial lesions and to plan the correct approach for the intervention. Although vascular imaging currently plays no clinical role of interest in the diagnosis of PAD, early imaging of PAD might be beneficial to determine a reliable starting point and the best therapeutic options for patients with PAD, but also to monitor the effects of vessel-stimulating therapies, to increase our knowledge concerning the biological processes and perhaps to help in the early identification of possible non-responders to therapy. MRI might be an ideal imaging solution for such an approach of 
patients with PAD, as MRI is a highly reliable tool to evaluate vascular morphology, but also vascular function during a single examination.

This thesis addresses the development and usage of morphologic and functional vascular MRI in patients with PAD. The main objectives were to determine and optimize the suitability of morphologic and functional vascular MRI in diagnosis and longitudinal follow up of PAD patients. Therefore, in this thesis different morphologic and functional vascular MRI techniques were tested as far as reproducibility and discriminative capability was concerned, and based upon these results improvements to existing techniques have been proposed and studied to increase reliability and to better differentiate between a diseased and healthy vasculature. Also, a standardized quantititive approach to acquire morphologic and functional vascular information was developed.

In chapter 2 the reproducibility of morphologic and functional vascular MRI was evaluated in PAD patients and healthy control subjects. In this study, quantification of the morphological (macro)vascular status by contrast-enhanced MR angiography, as well as phase contrast MRI to assess macro vascular blood flow proved highly reproducible in both PAD patients and healthy volunteers. These MR techniques might therefore be helpful in studying the development of collateral arteries in PAD patients and in unraveling the mechanisms underlying this process. Functional assessment of the microvascular status using dynamic contrast enhanced (DCE) and dynamic blood oxygen level-dependent (BOLD) imaging MRI were not reproducible and are therefore currently not suitable for clinical application in PAD.

In chapter 3 arterial peak flow (APF) measurements at different levels of the peripheral arterial vasculature in a large population of PAD and non-PAD patients were evaluated to determine the best location for APF measurements in order to facilitate clinical standardization. When measured at the level of the popliteal artery, the APF showed the largest differences between PAD and non-PAD patients, best discriminative capability and highest success rates of the measurement as compared to the APF acquired in the common or superficial femoral artery. Therefore 
the popliteal artery seems to be the most suitable level for a standardized flow measurement in PAD.

In chapter 4 the success rates and discriminative capability of MRI derived APF measurements were compared with conventional ankle brachial index $(\mathrm{ABI})$ measurements in a large clinical population of PAD patients. Compared to assessment of PAD severity with $A B I$, the success rate of MRI-based APF measurements was $20 \%$ higher (91\% versus $71 \%$ for APF and ABI measurements, respectively), with a similar discriminatory capacity for disease severity. We conclude that APF is a valuable measure to objectively assess PAD severity in patients in whom ABI cannot be reliably determined, which is especially convenient in patients already scheduled to undergo MR angiography.

In chapter 5 normative values for the APF in patients with PAD and non-PAD subjects were established and the influence of disease severity (intermittent claudication versus critical ischemia), presence of diabetes mellitus and age upon the APF were evaluated. Our reference values may aid in objective functional quantification of PAD severity and follow-up after intervention. We found that use of absolute flow values allows diagnosis of $\geq 50 \%$ luminal narrowing in the peripheral vascular tree with high accuracy.

In chapter 6 the results of a longitudinal follow-up study using CE-MRA and quantitative phase contrast imaging to study the effect of supervised exercise therapy on vascular changes in PAD patients were presented. Supervised exercise therapy in patients with intermittent claudication resulted in an increase in pain free walking distance, whereas with MRI no macrovascular adaptations in terms of additional collateral artery formation or flow increases were observed.

In chapter 7 a technique was presented to measure arterial flow reserve by quantitative $\mathrm{PCl}$ to improve the sensitivity of the APF to differentiate between PAD patients and healthy controls. Quantitative 2D MR cine $\mathrm{PCl}$ proved a promising method to determine flow reserve measures in patients with PAD and can be helpful to objectively discriminate patients with intermittent claudication from healthy controls. 
In chapter 8 we demonstrated the feasibility of DCE MRI of the calf musculature of PAD patients and healthy control subjects using a blood pool contrast agent. Using DCE MRI after contrast-enhanced MR angiography with gadofosveset enables reproducible assessment of hyperemic fractional microvascular blood plasma volume of the calf musculature. This hyperemic fractional microvascular blood plasma volume was lower in PAD patients than in healthy controls, which reflects a promising functional (hemodynamic) biomarker in the study of microvascular impairment in patients with PAD. 
Samenvatting 
APPENDIX 
Perifeer arterieel vaatlijden (PAV) is een expressie van algehele arteriosclerose met een gecompromitteerde arteriële doorbloeding van de onderste extremiteiten als gevolg van obstructieve perifere arteriële laesies. De symptomen variëren van claudicatio intermittens, doorgaans gedefinieerd als pijn en/of kramp van de kuitspieren in reactie op inspanning welke verdwijnt na een korte periode van rust, tot kritische ischemie van de ledematen, gedefinieerd als ischemische rust pijn, zweren en gangreen.

Arteriële laesies kunnen zich presenteren zowel op het macrovasculaire niveau (dat wil zeggen laesies in grote aanvoerende arteriën) als op het microvasculaire niveau, waarbij kleine arteriën, arteriolen en capillairen zijn aangedaan. Microvasculaire pathologie is het meest uitgesproken in de subgroep van patiënten met diabetes mellitus, terwijl macrovasculaire pathologie de belangrijkste oorzaak van de symptomen is bij niet-diabetische patiënten met PAV.

De vorming van collateraal arteriën is een van de mechanismen waarmee het menselijk lichaam kan compenseren voor de ischemie die uit deze obstructieve arteriële laesies voortvloeit. Echter, collateraalvorming is een ingewikkeld proces, waarvan men denkt dat het zowel de aanpassing van de bestaande arteriën als de vorming van nieuwe collaterale arteriën omvat. De omstandigheden waaronder deze collateraalvorming plaatsvindt bij patiënten met PAV is nog altijd een groot vraagstuk.

Momenteel wordt de diagnose van PAV meestal gesteld op basis van de typische klinische voorgeschiedenis, palpatie van de enkel pulsaties en door de meting van de enkel-arm index (EAl). Beeldvorming van het perifere vaatstelsel wordt alleen toegepast op die patiënten bij wie invasieve behandeling wordt overwogen, met als doel arteriële laesies te lokaliseren en de juiste benadering voor de interventie te plannen. Hoewel vasculaire beeldvorming geen klinische rol van betekenis speelt bij het diagnosticeren van PAV, kan vroege beeldvorming van PAV voordelig zijn om een betrouwbare uitgangsituatie vast te stellen en om de beste therapeutische opties te bepalen. Daarnaast is beeldvorming ook geschikt voor het monitoren van deze patiënten om de invloed 
van vaat stimulerende therapieën te evalueren en om onze kennis over de betrokken biologische processen te vergroten. Dit kan uiteindelijk misschien helpen bij de vroegtijdige identificatie van mogelijke nonresponders op therapie. MRI kan een ideale beeldvormende modaliteit zijn voor een dergelijke benadering van patiënten met PAV, aangezien MR angiografie een zeer betrouwbaar hulpmiddel is om de vasculaire morfologie evalueren en MRI daarnaast ook geschikt is om functionele metingen te verrichten.

Dit proefschrift richt zich op de ontwikkeling en het gebruik van morfologische en functionele vasculaire MRI metingen bij patiënten met PAV. De belangrijkste doelstellingen waren het bepalen en optimaliseren van de geschiktheid van morfologische en functionele vasculaire MRI metingen in de diagnostiek en longitudinale follow-up van patiënten met PAV. Daartoe zijn in dit proefschrift verschillende morfologische en functionele vasculaire MRI technieken getest op gebied van reproduceerbaarheid en discriminerend vermogen tussen vaatstelsels met en zonder PAV en op basis van deze resultaten zijn verbeteringen doorgevoerd aan de bestaande methoden om de betrouwbaarheid en het onderscheidend of discriminerend vermogen te verhogen. Ook werd een gestandaardiseerde, kwantitatieve methode voor het verkrijgen van morfologische en functionele vasculaire informatie ontwikkeld.

In hoofdstuk 2 van deze thesis werd de reproduceerbaarheid van morfologische en functionele vasculaire MRI metingen geëvalueerd in patiënten met PAV en gezonde proefpersonen, en werd gekeken naar de verschillen tussen patiënten en gezonde proefpersonen. In deze studie werd de morfologische (macro)vasculaire status gekwantificeerd door het tellen van collateraalvaten die afgebeeld werden met contrastversterkte MR angiografie. Daarnaast werd het functioneren van de vaten gekwantificeerd met behulp van fase-contrast (bloedsnelheid sensitieve) MRI. Beide methoden bleken zeer reproduceerbaar, zowel bij patiënten met PAV als gezonde vrijwilligers. Deze MR technieken kunnen daarmee nuttig zijn bij het bestuderen van de ontwikkeling van collateraalarteriën in patiënten met PAV en hulp bieden bij het ontrafelen van de mechanismen die ten grondslag liggen aan dit proces. De functionele 
kwantificatie van de microvasculaire status met gebruik van dynamische contrast-versterkte (DCE) en blood oxygen level-dependent (BOLD) MRI bleek slecht reproduceerbaar en is momenteel dan ook niet geschikt voor klinische toepassing in patiënten met PAV.

In hoofdstuk 3 werden arteriële peak flow (APF) metingen op verschillende fysieke niveaus van het perifere arteriële vaatstelsel in een grote populatie van patiënten met en zonder PAV geëvalueerd, om de beste locatie voor APF metingen te bepalen en zo standaardisatie van de meting te bevorderen. Hieruit bleek dat metingen op het niveau van de arterie poplitea resulteerden in de grootste verschillen tussen patiënten met en zonder PAV, het beste discriminerende vermogen en de hoogste slagingspercentages. Daarmee lijkt de arterie poplitea de meest geschikte locatie voor een gestandaardiseerde flowmeting te zijn in patienten met PAV.

In hoofdstuk 4 werden de slagingspercentages en het discriminerend vermogen van APF metingen vergeleken met conventionele EAI metingen in een grote klinische populatie van patiënten met PAV. Het slagingspercentage van APF metingen was $20 \%$ hoger ( $91 \%$ versus 71 $\%$ voor respectievelijk APF en EAl metingen), met een vergelijkbaar discriminerend vermogen voor de ernst van de ziekte. APF is daarmee een waardevolle meting om objectief de ernst van de ziekte vast te stellen bij patiënten met PAV bij wie de EAI niet of niet betrouwbaar kan worden bepaald.

In hoofdstuk 5 werden karakteristieke waarden voor de APF bij patiënten met en zonder PAV vastgesteld en de invloed van de ernst van ziekte (claudicatio intermittens versus kritische ischemie), de aanwezigheid van diabetes mellitus en de leeftijd op de APF geëvalueerd. De bepaalde referentiewaarden kunnen bijdragen aan de objectieve functionele kwantificering van de ernst van het PAV en de follow-up na de interventie.

In hoofdstuk 6 werden de resultaten gepresenteerd van een longitudinale follow-up studie van patiënten met PAV die gesuperviseerde looptherapie ondergingen en waarbij met behulp van contrast- 


\section{APPENDIX}

versterkte MR angiografie en kwantitatieve flow metingen het effect van deze therapie op de bloedvaten werd bestudeerd. Gesuperviseerde looptherapie bij deze patiënten met claudicatio intermittens resulteerde in een toename van de pijnvrije loopafstand, terwijl met MRI geen macrovasculaire veranderingen werden waargenomen.

In hoofdstuk 7 werd een techniek gepresenteerd om de arteriële flowreserve te meten en zodoende gevoeligheid van de APF meting om te discrimineren tussen patiënten met PAV en gezonde personen te verbeteren.

In hoofdstuk 8 toonden we de mogelijkheid om met behulp van een 'blood pool' contrastmiddel DCE MRI van de kuitspieren bij patiënten met PAV en gezonde proefpersonen te verrichten. Door de DCE MRI meting te verrichten na contrast-versterkte MR angiografie met gadofosveset als contrastmiddel, werd het mogelijk om een reproduceerbare bepaling van het hyperemic fractional microvascular blood plasma volume van de kuitspieren te bepalen. Dit hyperemic fractional microvascular blood plasma volume bleek bij patiënten met PAV lager dan bij gezonde personen, waarmee het een veelbelovende functionele (hemodynamische) biomarker is voor de studie naar microvasculaire pathologie bij patiënten met PAV. 
Dankwoord 
APPENDIX 
De studies beschreven in dit proefschrift waren nooit mogelijk geweest zonder de medewerking van vele patiënten en vrijwilligers, die bereid waren een (vaak) langdurig en een niet al te comfortabel MRI onderzoek te ondergaan, in veel gevallen zelfs meerdere malen. Daarom wil ik in eerste instantie al deze personen, die vaak belangeloos deelnamen, danken voor hun belangrijke bijdrage aan de totstandkoming van dit proefschrift!

(Prof?) Dr. Tim Leiner. Beste Tim, jij hebt mij de mooie kanten van wetenschappelijk onderzoek doen laten zien. Je hebt mij vanaf de eerste dag van onze samenwerking het gevoel van vertrouwen gegeven en ik ben je zeer dankbaar voor alle kansen die je me hebt geboden. Samenwerken met zo'n gemotiveerd en gedreven persoon als jij was voor mij een hele prettige en leerzame ervaring. Ondanks je, destijds voor mij onverwachte, vertrek naar Utrecht stond dit onze samenwerking gelukkig niet in de weg. Wie weet zullen we in de toekomst nog vaker een project samen doen.

Prof. Dr. Ir. Walter Backes. Beste Walter, na Tim's vertrek naar Utrecht waren we voor dit onderzoek toch vooral op elkaar aangewezen. Een fysicus en een arts, die niet zelden anders tegen de onderwerpen aan keken. Desondanks hebben we het onderzoek tot een goed einde weten te brengen en ik ben je dankbaar voor de samenwerking in de afgelopen jaren.

Dr. Patty Nelemans. Beste Patty, ook jouw bijdrage aan dit onderzoek was van groot belang. Niet zelden dachten we het ei van Columbus ontdekt te hebben, waarna jouw berekeningen en inzichten ons weer met beide benen op de grond zette. Hoewel dat nog wel eens wat frustratie met zich mee bracht, kwamen de manuscripten door jouw inbreng op een kwalitatief hoger niveau. Ik ben je daarom erg dankbaar voor je bijdrage aan dit proefschrift en ben blij dat je een van mijn copromotoren bent. 
Prof. Dr. Joachim Wildberger. Beste Joachim, met uw komst naar Maastricht raakte $u$ kort na de start van mijn onderzoek betrokken bij dit project. Hoewel er in het begin enige bedenkingen waren over de doelstellingen en haalbaarheid, heeft dit onderzoek mede dankzij uw inspanningen geleid tot een heel aantal mooie publicaties. Ik ben $\mathrm{u}$ erg dankbaar voor deze samenwerking en de waardering die u menigmaal heeft uit gesproken over mijn werk.

Prof. Dr. Michiel de Haan. Beste Michiel, hoewel jij nooit direct betrokken bent geweest bij mijn studies, ben ik je dankbaar voor het vertrouwen dat je me hebt gegeven vanaf het eerste moment dat ik op de afdeling Radiologie van het azM rond liep. In de afgelopen jaren ben ik gemarineerd geraakt in de MRA's en CTA's en hebben we leuke verbeteringen aan protocollen en beeldkwaliteit op het gebied van MRA weten door te voeren. Je interesse in de voortgang van het onderzoek en je luisterend oor in de mindere tijden heb ik altijd zeer gewaardeerd. Hoewel onze wegen zich ondertussen waarschijnlijk gescheiden hebben, kijk ik met veel plezier terug naar onze samenwerking in de afgelopen jaren.

MRI laboranten azM. Beste laboranten, ook zonder jullie medewerking was dit onderzoek niet mogelijk geweest. Thea en Etienne, langs deze weg wil ik jullie speciaal hartelijk danken voor het feit dat jullie altijd bereid waren mee te denken, nieuwe protocollen uit te testen en door te voeren, ook buiten werktijd.

Afdeling vaatchirurgie azM. I $\mathrm{k}$ wil de afdeling vaatchirurgie, en dan met name prof. dr. Schurink en dr. Daemen hartelijk danken voor de samenwerking en hun inspanningen om patiënten te werven voor deelname aan de verschillende studies. Zonder deze samenwerking was dit onderzoek niet mogelijk geweest. 
Pap, mam, Peter, ik wil jullie hartelijk danken voor jullie interesse en medewerking (ook jullie zijn immers voor mij in die scanner gedoken) in mijn onderzoek in de afgelopen jaren. Jullie hebben me zien veranderen van een heel gemotiveerde promovendus naar iemand die erg kritisch is geworden en van bepaalde keuzes spijt heeft gekregen; daar hebben we vele discussies over gevoerd. Nu dit hoofdstuk afgesloten is, is het tijd voor een nieuwe, totaal andere uitdaging in een totaal andere wereld.

Michel, ik heb geen idee hoelang wij elkaar nu kennen (kan me in ieder geval nog een oude Dell \& Carmageddon(?) herinneren, wat een tijd was dat...), maar onze vriendschap heeft de nodige vrouwen, studies en nog meer verhuizingen weten te doorstaan. Of het nu voor goed gesprek of een nieuw spel is (al dan niet met blauwe legers), we weten elkaar gelukkig altijd weer te vinden. Hoewel je eerste ervaring met de MRI niet helemal jouw ding bleek te zijn, ben ik blij dat mee wilde werken. Bedankt voor je vriendschap en je interesse in dit onderzoek. We zien elkaar snel weer in Veldhoven... of Nijmegen... of Veldhoven... of Nijmegen... ('t is altijd weer een verrassing waar je woont).

Jeroen, een speciaal woord van dank voor jou, voor onze vriendschap, je belangstelling in mijn onderzoek, het mee denken en de vele discussies die we hebben gevoerd 'ons wereldje'. Ik ken weinig mensen die zo enthousiast zijn in hun bezigheden, die zich zo (belangeloos) inzetten en zo in en in goed zijn als jij. Ik hoop dat onze vriendschap ook na mijn tijd in het Limburgse nog lang zal bestaan.

Marloes... wat hebben wij een hectische tijd achter de rug. Wij leerden elkaar kennen op het moment dat bij mij de twijfels het grootst waren, maar jij wist me met je vlotte (en niet te stoppen) babbel altijd weer het positieve van dingen in te laten zien. Bedankt voor je steun, je vriendschap en de gezellige tijd die we hebben gehad en hopelijk nog gaan hebben! 


\section{APPENDIX}

Alice. Lieve Alice, ik kan niet beschrijven hoe mijn leven is veranderd sinds ik jou heb leren kennen en hoeveel vreugde jij hebt gebracht in mijn leven. Hoewel je dit bepaald niet als je grootste of beste daad beschouwt, heb jij mij doen beseffen dat er veel belangrijkere dingen zijn in het leven dan werk, maar vooral wat echte waardering is. Je bent mijn vriendin, mijn maatje, mijn geweten en sinds dit jaar ook nog eens mijn zakenpartner! Of het nu binnen de radiologie is of met ons eigen bedrijfje (manege?) of allebei, wij gaan het samen maken lieve schat, is het niet hier, dan wel in ons geliefde Zweden. Ik hou van je! 
Curriculum vitae 
APPENDIX 


\section{Curriculum vitae}

\section{Personal details:}

Name: Bastiaan Versluis

Nationality: Dutch

Date of birth: Februari 24, 1983

Place of birth: Eindhoven, the Netherlands

Overview education, qualifications and work:

1995-2001 High school, Christiaan Huygens College, Eindhoven, the Netherlands; Gymnasium (Cum Laude)

2001-2007 Medicine, Maastricht University, Maastricht, the Netherlands (Cum Laude)

2007-2008 Researcher, Clinical Trial Center Maastricht (CTCM), Maastricht, the Netherlands

2008-2014 PhD student, Department of Radiology, Academic Hospital Maastricht (azM)/Maastricht University Medical center (MUMC), Maastricht, the Netherlands

2010- present Resident in Radiology, Department of Radiology, azM, Maastricht, the Netherlands

2013-2014 Co-founder Dutch Export Horses (DEH), Maastricht, the Netherlands

2014- present Co-founder/co-owner Versluis-Huisman V.O.F. |International Horse Trade Center (IHTC.eu), Maastricht, the Netherlands 
APPENDIX 
Publications 
APPENDIX 


\section{Publications in Peer Reviewed Journals}

1. Versluis B, Tuinder S, Boetes C, Van Der Hulst R, Lataster A, Van Mulken T, Wildberger J, de Haan M, Leiner T. Equilibrium-phase high spatial resolution contrast-enhanced MR angiography at 1.5T in preoperative imaging for perforator flap breast reconstruction. PloS One 2013;8(8):e71286

2. Leiner T, Habets J, Versluis B, Geerts L, Alberts E, Blanken N, Hendrikse J, Vonken EJ, Eggers H. Subtractionless first-pass single contrast medium dose peripheral MR angiography using two-point Dixon fat suppression. Eur Radiol. 2013;23(8):2228-35

3. Versluis B, Leiner T, Nelemans PJ, Wildberger JE, Schurink GW, Backes WH. Magnetic resonance imaging-based monitoring of collateral artery development in patients with intermittent claudication during supervised exercise therapy. J Vasc Surg. 2013 Nov;58(5):1236-43.

4. Versluis B, Dremmen MH, Nelemans PJ, Wildberger JE, Schurink GW, Leiner T, Backes WH. Dynamic contrast-enhanced MRI assessment of hyperemic fractional microvascular blood plasma volume in peripheral arterial disease: initial findings. PLoS One. 2012;7(5):e37756.

5. Versluis B, Dremmen MH, Nelemans PJ, Wildberger JE, Schurink GW, Leiner $\mathrm{T}$, Backes WH. MRI of arterial flow reserve in patients with intermittent claudication: feasibility and initial experience. PLoS One. 2012;7(3):e31514.

6. Jaspers K, Versluis B, Leiner T, Dijkstra P, Oostendorp M, van Golde JM, Post MJ, Backes WH. MR angiography of collateral arteries in a hind limb ischemia model: comparison between blood pool agent Gadomer and small contrast agent Gd-DTPA. PLoS One. 2011 Jan 26;6(1):e16159. 


\section{APPENDIX}

7. Versluis B, Backes WH, van Eupen MG, Jaspers K, Nelemans PJ, Rouwet EV, Teijink JA, Mali WP, Schurink GW, Wildberger JE, Leiner T. Magnetic resonance imaging in peripheral arterial disease: reproducibility of the assessment of morphological and functional vascular status. Invest Radiol. 2011 Jan;46(1):11-24.

8. Gerretsen SC, Versluis B, Bekkers SC, Leiner T. Cardiac cine MRI: comparison of 1.5 T, non-enhanced 3.0 T and blood pool enhanced 3.0 T imaging. Eur J Radiol. 2008 Jan;65(1):80-5.

9. Versluis B, Nelemans P.J, Brans R, Wildberger J.E, Schurink G.W.H, Leiner T, Backes W.H. Functional MRI in peripheral arterial disease: Arterial peak flow versus ankle-brachial index. PLoS One. 2014 Feb 5;9(2):e88471.

10. Versluis B, Leiner T, Nelemans P.J, Brans R, Wildberger J.E, Schurink G.W.H, Backes W.H. MRI derived arterial peak flow in peripheral arterial disease: Towards a standardized measurement. Accepted for publication in the European Journal of Vascular and Endovascular Surgery.

11. Versluis B, Backes W.H, Nelemans P.J, Brans R, Wildberger J.E, Schurink G.W.H, Leiner T. Reference values for peripheral arterial peak flow in patients with peripheral arterial disease: Assessment by phase-contrast magnetic resonance flow imaging. Submitted. 

